\title{
CANONICAL DESCRIPTION OF GROUP THEORY: A LINEAR ORDER ON ALL FINITE GROUPS
}

\author{
Juan Pablo Ramírez \\ Centro de Investigación en Matemáticas (CIMAT), Guanajuato 36023, \\ Mexico; juan.rmz236@gmail.com;
}

\begin{abstract}
We provide a construction of natural numbers that is unique with respect to other constructions, and use this construction in the domain of algebra and finite functions to find several results in finite group theory. First, we give a linear order to the set of all finite functions. This gives a linear order in the subset of all finite permutations. To do this, we assign a unique natural number, $N_{f}$, to every finite function $f$. The sub order on permutations is well defined with respect to cardinality; if $\eta_{m}, \eta_{n}$ are permutations on $m<n$ objects, then $N_{\eta_{m}}<N_{\eta_{n}}$. This representation also has the characteristic $N_{\mathbf{1}_{n}}<N_{\eta}<N_{\text {id }_{n}}$ where $\mathbf{1}_{n}$ is the one-cycle permutation of $n$ objects, $\mathbf{i d}_{n}$ is the identity permutation of $n$ objects, and $\eta$ is any permutation of $n$ objects. This representation provides a good definition of equivalent functions, and equivalent objects on functions. We are able to do this for both concrete functions, and abstract functions. We use this in the main section, on group theory, to number the set of all finite groups. We are able to well represent every finite group as a natural number; two groups are represented by the same natural number if and only if they are in the same isomorphism class. In fact, we are able to give a linear order to the set of finite groups. Specifically, we give a canonical bijective function $\mathbf{G}_{\text {Fin }} \rightarrow \mathbb{N}$. This representation, $N_{G}$, of $G$, is also well behaved with respect to cardinality. Additionally, the cyclic group $\mathbb{Z}_{n}$ has smaller representation than any group of $n$ objects, and the group with largest representation is the abelian group $\mathbb{Z}_{p_{1}}^{n_{1}} \oplus \mathbb{Z}_{p_{2}}^{n_{2}} \oplus \cdots \oplus \mathbb{Z}_{p_{k}}^{n_{k}}$, where $n=p_{1}^{n_{1}} p_{2}^{n_{2}} \cdots p_{k}^{n_{k}}$ is the prime factorization of $n$. This representation of a finite group as a natural number also provides a linear order to the elements of the group, arranging its Cayley table in a canonical block form. The last section is an introductory description of real numbers as infinite sets of natural numbers. Real functions are represented as sets of real numbers, and sequences of real functions $f_{1}, f_{2}, \ldots$ are well represented by sets of real numbers, as well. In the last section we well assign mathematical objects to tree structures and conclude with some brief comments on type theory and future work. In general we are able to represent and manipulate mathematical objects with the smallest possible type, and minimum complexity.
\end{abstract}

key word: Finite Groups; Finite Permutations; Type Theory; Set Theory; Mathematical Structuralism; Trees

\section{Introduction}

The present work is part of a broader attempt in proposing an optimal universe for classical mathematics. The construction presented in [Ramirez(2019)], is the first exposition of natural and real numbers, defined as set numbers. Here, we focus on finite structures, and group theoretic aspects of this proposal. The constructions are self contained, and we go into detail of some constructions and proofs. We begin by giving an adequate description of algebraic structures. We take an approach in the definition of operation, group, field, and linear space that will allow the constructions of the next sections. These definitions where initially explored in [Ramirez(2015)], but we have made an attempt to thoroughly revise the exposition. In the second 
section, we proceed with a description of natural numbers as the set of hereditarily finite sets, HFS. This is a more axiomatic treatment, and details for proofs not given in [Ramirez(2019)] are given here. An order $<$ and operation $\oplus$ is given, on HFS, that is isomorphic to $\mathbb{N}$. This is a canonical representation of natural numbers in set theory. Natural numbers are elements of HFS in such a way that Von-Neumann and Zermelo-Fraenkel ordinals are both embedded sub orders of our construction of $\mathbb{N}$. In the third section we provide a method of representing a finite function as a natural number. If $A, B$ are two finite sets, and $f: A \rightarrow B$ a function, we are able to well assign a natural number to that function. Two finite functions have the same structure if they are assigned the same natural number. Then, we focus on the formal definition of finite groups. We give the definition of canonical form for a group, where a single natural number is used to represent the group. This reduces the problem of proving two finite groups are isomomorphic, to finding the canonical representation of these groups and compare these natural numbers. We provide the method for finding groups of order $n$, and finding their canonical representation. We find the linear order for all groups with $|G|<10$.

$$
\mathbb{Z}_{1}<\mathbb{Z}_{2}<\mathbb{Z}_{3}<\mathbb{Z}_{4}<K(4)<\mathbb{Z}_{5}<\mathbb{Z}_{6}<D_{6}<\mathbb{Z}_{7}<\mathbb{Z}_{8}<Q_{8}<D_{8}<\mathbb{Z}_{2} \oplus \mathbb{Z}_{4}<\mathbb{Z}_{2}^{3}<\mathbb{Z}_{9}<\mathbb{Z}_{3}^{2}<\cdots,
$$

where $\mathbb{Z}_{n}$ is the cyclic group of order $n, K(4)$ is the Klein 4-group, $D_{2 n}$ is the Dihedral group, $Q_{8}$ is the quaternion group, and $\oplus$ is direct product. For example, $\mathbb{Z}_{2}^{3}=\mathbb{Z}_{2} \oplus \mathbb{Z}_{2} \oplus \mathbb{Z}_{2}$.

Then, an overview of the infinite case of these results, which is a model for real numbers, is given. We mention a general outline on treating concepts of calculus, which will be described in full in a separate publication. The study of real numbers is reduced to the study of natural numbers. However, the gap (conceptual and practical) between these two kinds of objects is enormous, in most axiomatic treatments. Our construction of natural numbers, will allow us to express the continuum of real numbers as an extension of natural numbers. It is not necessary to build intermediate structures such as $\mathbb{Z}$ or $\mathbb{Q}$, although we do provide descriptions of these structures also. Just as we are able to reduce a finite group to a natural number, we will have some similar results in the infinite case. For example, we are able to express a real function as a set of real numbers. More surprisingly, a sequence of real functions is also a set of real numbers. The general idea is that we can reduce the complexity of objects to its minimum possible. In the last section we will express mathematical objects using tree structures. We will see that natural numbers are finite trees, real numbers will be infinite trees and we will give a general description of mathematical objects. We will see how this gives us a theory of types.

\section{Groups, Fields and Linear Spaces}

The main difference with the algebraic methods we will use here, is to replace quotient groups with groups of automorphisms in the description of group theory. Numerical systems have, traditionally, been described in terms of quotient spaces. We take an alternate approach by defining the operation of a group as a function $X \rightarrow(X \rightarrow X)$. We give a description of fields and a general definition of a vector space as a field of automorphisms for an abelian group. We provide necessary and sufficient conditions of a linear space. Our definitions of group enables us to have trivial proofs of our theorems, in the theory of set numbers. In order to prove the statements of [Ramirez(2019)], we must reformulate our algebraic foundations.

Definition 1. Let $G$ a non empty set, and Aut $G$ be the set of bijective functions of the form $G \rightarrow G$. A function $G \rightarrow$ Aut $(G)$ is called an operation on the set $G$.

A set of functions $B \subseteq$ Aut $G$ is said to be balanced if id $_{G} \in B$, and if $x \in B$ implies $x^{-1} \in B$. Let $*: G \rightarrow B$ a bijective function, for some balanced set $B$. If $*$ satisfies

$$
*(x) \circ *(y)=*(*(x)(y))
$$

we say it is a group structure. 
The functions $*(x)$ are called operation functions of $*$. We remark that the expression $*(x)(y) \in G$ is the image of $y$ under the action of $*(x)$. Thus, $*(*(x)(y)) \in$ Aut $G$ is the image of $*(x)(y) \in G$ under the action of $*$.

Theorem 1. Given a group structure, we are able to construct a group. The operation of the group is defined by $a * b=*(a)(b)$. Given a group $G$ we can construct a group structure, *.

Proof. - Identity Element

There exists an object $e \in G$ such that $*(e)=\mathbf{i d}_{G}$. Therefore, $*(e)(x)=x$ for all $x \in G$. This means $e * x=x$ for all $x \in G$. Now we have to prove $x * e=x$. We have $*(*(x)(e))=*(x) \circ *(e)=*(x)$. Since $*$ is injective, $*(x)(e)=x$.

- Inverse Element

Let $a \in G$, then there exists a unique $a^{-1} \in G$ such that $*\left(a^{-1}\right)=(*(a))^{-1}$ is the inverse function of $*(a)$. This is a direct consequence of the definition of balanced set. We must show $a * a^{-1}=a^{-1} * a=e$. It is enough to prove $a^{-1} * a=e$. We know $a^{-1} * a=*\left(a^{-1}\right)(a)=(*(a))^{-1}(a)$. But, $*(a)(e)=a$. This means $(*(a))^{-1}(a)=e$.

- Associativity

$$
\begin{aligned}
x *(y * z) & =*(x)(y * z) \\
& =*(x)(*(y)(z)) \\
& =(*(x) \circ *(y))(z) \\
& =*(*(x)(y))(z) \\
& =(*(x)(y)) * z \\
& =(x * y) * z .
\end{aligned}
$$

We use the equivalence of groups and group structures to find the basic properties of groups.

Theorem 2. Let $G(*)$ a group with operation $*$. Then, we verify

1. Right cancellation; $*(a)(c)=*(b)(c)$ implies $a=b$.

2. Left cancellation; $*(c)(a)=*(c)(b)$ implies $a=b$.

3. Uniqueness of identity and inverse elements.

4. Inverse of inverse; $\left(x^{-1}\right)^{-1}=x$.

5. Existence of unique solutions; given $a, b \in G$ there exists a unique $x \in G$ such that $*(a)(x)=b$, and $a$ unique $y \in G$ such that $*(y)(a)=b$.

Proof. The first part requires to apply the function $*$, so that $*(*(a)(c))=*(*(b)(c))$ which implies $*(a) \circ *(c)=*(b) \circ *(c)$. Right cancellation of functions gives $*(a)=*(b)$. We conclude $a=b$ because $*$ is bijective. We can similarly prove the second part if we use left cancellation of functions.

Let $e_{1}, e_{2}$ be identity elements. If we consider $e_{1}$ as identity we get $*\left(e_{1}\right)\left(e_{2}\right)=e_{2}$, and if we consider $e_{2}$ identity we get $*\left(e_{1}\right)\left(e_{2}\right)=e_{1}$. Therefore $e_{1}=e_{2}$. The uniqueness of the inverse is trivial. If $a_{1}, a_{2}$ are inverse elements of $a$, then $* a\left(a_{1}\right)=e=* a\left(a_{2}\right)$ implies $a_{1}=a_{2}$ because of left cancellation. 
Let $y=x^{-1}$, so that $*(x)$ and $*(y)$ are inverse functions; $(*(x))^{-1}=*(y)$ and $(*(y))^{-1}=*(x)$. The inverse element of $y=x^{-1}$ is the object $z$ such that $*(z)$ is the inverse function of $*(y)$. Therefore, $x$ is the inverse of $y$ and we conclude $\left(x^{-1}\right)^{-1}=x$.

For the last part, consider $a, b$ fixed. We know $*(a)$ is a bijective function $G \rightarrow G$ so that their exists a unique $x \in G$ such that $*(a)(x)=b$. On the other hand, we would like to find a function $*(y)$ that sends $a$ to $b$. We see that $b *\left(a^{-1} * a\right)=b$, which can be rewritten as $\left(*(b) \circ *\left(a^{-1}\right)\right)(a)=b$. Our function is $*(y)=*\left(b * a^{-1}\right)$. Suppose we have a second object $w$ that satisfies the property of $y$. Then $*(y)(a)=*(w)(a)$ which is equivalent to $y * a=w * a$ which in turn implies $y=w$ if we use right cancellation.

Proposition 1. A group structure, *, defines a new function $\bar{*}: G \rightarrow \operatorname{Aut}(G)$ such that $\bar{*}(a)(b)=*(b)(a)=b * a$. The function $\bar{*}$ is also a group structure. The two group structures $*, *$ are equivalent in the sense that they both generate isomorphic groups.

Proof. Let us first prove $\bar{*}$ is a group structure. We must show $\bar{*}$ is an injective function $\bar{*}: G \rightarrow B$, where the image $I m \bar{*}=B$ is a balanced subset of $\operatorname{Aut}(G)$. Every object $a \in G$ is assigned a unique function $\bar{*}(a)$ and we easily find $\bar{*}(e)=\mathbf{i d}_{G}$. Next we prove $\bar{*}(a)$ is bijective. First of all, it is injective. Take $\bar{*}(a)(x)=\bar{*}(a)(y)$ which is equivalent to the expression $x * a=y * a$, then $x=y$ because of right cancellation. This proves $₹(a)$ is injective. Let us prove $\bar{*}(a)$ is onto $G$. Let $b \in G$, then there exists a solution $x$ to the equation $x * a=b$ which is equivalent to $\bar{*}(a)(x)=b$. This proves $\bar{*}(a)$ is a bijection. Now let us prove $\bar{*}\left(a^{-1}\right)=(\bar{*}(a))^{-1}$ is the inverse function of $\bar{*}(a)$. We know, by definition, $\bar{*}\left(a^{-1}\right)(x)=x * a^{-1}$. We also know $\bar{*}(a)$ acts by $\bar{*}(a)\left(x * a^{-1}\right)=\left(x * a^{-1}\right) * a=x$, which implies the inverse function $(\bar{*}(a))^{-1}$ acts by $(\bar{*}(a))^{-1}(x)=x * a^{-1}$. This proves $\bar{*}\left(a^{-1}\right)=(\bar{*}(a))^{-1}$. So far, we have proven the image of $\bar{*}$ is a balanced set.

To prove $\bar{*}$ is injective, take two objects $x, y \in G$ such that $\bar{*}(x)=\bar{*}(y)$. Then, $x=\bar{*}(x)(e)=\bar{*}(y)(e)=y$. Now we prove the associative property holds. For all $a, b$ we have

$$
\begin{aligned}
\bar{*}(\bar{*}(a)(b))(x) & =\bar{*}(b * a)(x) \\
& =x *(b * a) \\
& =(x * b) * a \\
& =\bar{*}(a)(x * b) \\
& =\bar{*}(a)(\bar{*}(b)(x)) \\
& =(\bar{*}(a) \circ \bar{*}(b))(x),
\end{aligned}
$$

for all $x \in G$. We have proven $\bar{*}$ is a group structure. Let $G(*)$ be the group generated by $*$ and $G(\bar{*})$ the group generated by $\bar{*}$. These two groups are isomorphic by $x \mapsto x^{-1}$. To prove, take $\phi(a * b)=(a * b)^{-1}=b^{-1} * a^{-1}=\phi(b) * \phi(a)=\phi(a) * \phi(b)$.

Definition 2. In general the functions $*(x)$ and $*(x)$ are not equal. When they are equal, we say the object $x$ commutes. A group is abelian if its two generating functions are equal, $*=\bar{*}$.

Proposition 2. Let $G(*)$ an operation on the set $G$. The following are equivalent statements.

1. The operation $*$ is associative.

2. $*(*(x)(y))=*(x) \circ *(y)$ is true for all $x, y \in G$.

3. $*(x) \circ \bar{*}(y)=\bar{*}(y) \circ *(x)$ for all $x, y \in G$. 
Proof. The equivalence of 1 . and 2. was proven in Theorem 1 . Now we prove the equivalence of 1 . and 3.

$$
\begin{aligned}
(*(x) \circ \bar{*}(y))(z) & =*(x)(\bar{*}(y)(z)) \\
& =*(x)(z * y) \\
& =x *(z * y) \\
& =(x * z) * y \\
& =\bar{*}(y)(x * z) \\
& =\bar{*}(y)(*(x)(z)) \\
& =(\bar{*}(y) \circ *(x))(z)
\end{aligned}
$$

If we suppose 3 . holds, then we can prove associativity,

$$
\begin{aligned}
x *(z * y) & =*(x)(z * y) \\
& =*(x)(\bar{*}(y)(z)) \\
& =(*(x) \circ \bar{*}(y))(z) \\
& =(\bar{*}(y) \circ *(x))(z) \\
& =\bar{*}(y)(*(x)(z)) \\
& =\bar{*}(y)(x * z) \\
& =(x * z) * y
\end{aligned}
$$

We have the following useful result, for consequent sections. It gives a practical means of proving associativity. If the elements of $G$ commute and the operation functions also commute, then the operation is associative.

Proposition 3. If $*$ is a commutative operation on the set $G$, and $*(x) \circ *(y)=*(y) \circ *(x)$, for all $x, y \in G$, then $*$ is associative.

Proof. Given our hypothesis, we have the equalities $*(x) \circ \bar{*}(y)=*(x) \circ *(y)=*(y) \circ *(x)=\bar{*}(y) \circ *(x)$. Our result follows from 3. and 1. of the last proposition.

Definition 3. Let $G(*)$ a group and let $H \subseteq G$ be a subset of the set $G$. Define $*_{H}$ as the function $*$ restricted to $H$. If $*_{H}$ is a group structure we say it is a subgroup of $G(*)$.

For $H \subset G$ to be a subgroup of $G$ it is necessary that the image of $H$, under the action of $*_{H}(h)$, be equal to $H$, for all $h \in H$. In short, $*_{H}(h)[H]=H$, for all $h \in H$. This means $H$ is closed under the operation $*$.

Definition 4. Given two groups $G_{1}\left(*_{1}\right)$ and $G_{2}\left(*_{2}\right)$, a homomorphism is a function $\phi: G_{1}\left(*_{1}\right) \rightarrow G_{2}\left(*_{2}\right)$ such that

$$
\phi\left(*_{1}(a)(b)\right)=*_{2}(\phi(a))(\phi(b))
$$

The set of all homomorphisms from $G_{1}\left(*_{1}\right)$ to $G_{2}\left(*_{2}\right)$ is represented by the notation $\boldsymbol{H o m}\left(G_{1}, G_{2}\right)$, when no confusion arises with respect to the operations of each group. 
If the homomorphism is injective as function then we call it a monomorphism, and if it is surjective as function we call it an epimorphism. If the function is bijective we have an isomorphism. Lastly, an automorphism is an isomorphism of the form $\phi: G \rightarrow G$. The set of all automorphisms of $G(*)$ is represented with the notation Aut $G(*)$.

We use the notation $\operatorname{Aut}(G)$ and Aut $G(*)$ to differentiate between bijective functions and automorphisms.

Theorem 3. Let $X$ a set, then the composition operation $\circ$ is a group structure for the set of all bijective functions Aut X. A subset $B \subseteq$ Aut $X$ that is balanced and closed under composition is a subgroup $B(\circ) \subset$ Aut $X$.

A group structure $*: G \rightarrow B$, provides an isomorphism $*: G(*) \rightarrow B(0)$.

The composition operation is a group structure for the set of automorphisms Aut G(*). A balanced and closed subset, $\mathcal{B} \subseteq$ Aut $G(*)$, is a subgroup $\mathcal{B}(\circ) \subset$ Aut $G(*)$.

Proof. For the first part, we have a function o : Aut $X \rightarrow \operatorname{Aut}(\operatorname{Aut} X)$. If $f \in \operatorname{Aut} X$, then $\circ(f):$ Aut $X \rightarrow$ Aut $X$ is the function that acts by $\circ(f)(g)=f \circ g$. We have to prove $\circ:$ Aut $X \rightarrow B$ is a bijective function, and the image $\operatorname{Im} \circ=B \subset$ Aut(Aut $X)$ is a balanced set. Every object in Aut $X$ is assigned a function $\circ(f) \in \operatorname{Aut}($ Aut $X$ ). To see $\circ$ is injective, take two objects $f, g \in$ Aut $X$ and suppose $\circ(f)=\circ(g)$. This implies $f=f \circ \mathbf{i d}_{X}=g \circ \mathbf{i d}_{X}=g$. Now we focus on the image of $\circ$. We first prove the objects in the image are bijective functions Aut $X \rightarrow$ Aut $X$. Suppose $\circ(f)(g)=\circ(f)(h)$, for two $g, h \in$ Aut $X$. That is to say, $f \circ g=f \circ h$ and because of cancellation of bijections, we have $g=h$. This means $\circ(f)$ is injective. To prove $\circ(f)$ is onto Aut $X$, take any $g \in$ Aut $X$, and we find $\circ(f)(x)=f \circ x=g$ for $x=f^{-1} \circ g$. We also know the image of $\circ$ is balanced because $\circ\left(\operatorname{id}_{G}\right) \in \operatorname{Aut}($ Aut $X)$ and $(\circ(f))^{-1}=\circ\left(f^{-1}\right) \in \operatorname{Aut}($ Aut $X)$. The associative property is the usual associativity of composition of functions. This proves the first assertion of the first part. The second assertion of the first part is trivial. Take $B(\circ)$ balanced and closed under composition. This makes $B(\circ)$ a subgroup.

For the second part, we must prove $*$ is an isomorphism. From the first part of this theorem we know $B(\circ)$ is a group. We also know $*$ is a bijection. We use definition 4 and associativity, in $G$, to verify $*(*(x)(y))=*(x) \circ *(y)=\circ(*(x))(*(y))$. This proves that the group structure $*$ produces an isomorphism $G(*) \rightarrow B(\circ)$, where $B(\circ)$ is the image of $*$ with the operation $\circ$.

The third part of this theorem is proven similarly to the first part of this theorem.

Definition 5. Let $G(*)$ a group and $X$ a set. A homomorphism $\phi: G(*) \rightarrow$ Aut $X$ is called a group action.

Some group actions take a special form that provides additional structure. For example, the image of the group action can be a set of homomorphisms on some fixed group. We will define the distributive property in terms of homomorphisms of the special form $G(*) \rightarrow \operatorname{Hom}(G, G)$. When we define a group structure, we have a bijective function $*: G \rightarrow B \subset \operatorname{Aut}(G)$ where $G$ is a set and $\operatorname{Aut}(G)$ is a set of bijective functions. Given the group $G(*)$, suppose we have a function, $G \rightarrow \mathbf{H o m}(G, G)$. Then we have what we commonly refer to as the distributive property.

Definition 6. Let $K(+)$ a group with identity 0 ; the set $K-\{0\}$ is represented by $K_{0}$. Let $\cdot: K_{0} \rightarrow C \subset \boldsymbol{H o m}(K, K)$ a group structure $K(\cdot)$. We say $K(\cdot)$ distributes over $K(+)$, which means

$$
\cdot(x)(+(a)(b))=+(\cdot(x)(a))(\cdot(x)(b)) .
$$

A field is an abelian group $K(+)$, together with a second abelian group $K(\cdot)$ that distributes over $K(+)$. The identity element, $1 \in K(\cdot)$ is referred to as the unit of the field.

We are saying the distributive property holds when we have a group, $K(\cdot)$, whose operation functions, $\cdot x$, are homomorphisms on another group $K(+)$. Our conditions give us the relations $\cdot(x)(0)=0$, for all $x \in K$. Therefore, we define $\cdot(0)(x)=0$. The function associated to 0 is the trivial function $\mathbf{0}: K \rightarrow\{0\}$. 
Given an abelian group, $V(\oplus)$, we are able to provide a second operation on the set of homomorphisms, apart from composition. The operation on the group naturally induces an operation on the homomorphisms of the group. This will allow us to define linear spaces, formally. Define addition of homomorphisms by $(f \oplus g)(x)=f(x) \oplus g(x)$. If $\mathcal{B} \subset$ Aut $V(\oplus)$ we write $\mathcal{B}(\oplus)$ to emphasize we are considering the set together with addition of automorphisms, not composition. The trivial function $\mathbf{e}: V \rightarrow\{e\}$ acts as an identity object under addition of homomorphisms because $f=f \oplus \mathbf{e}=\mathbf{e} \oplus f$. Let $f \in$ Aut $V(\oplus)$, and $-f \in$ Aut $V(\oplus)$ the automorphism defined by $-f(x)=-(f(x))$ where $-(f(x))$ is the additive inverse of $f(x)$; we use the notation $-x=x^{-1}$ for the inverse of $x$ under $\oplus$. We easily verify $f \oplus(-f)=\mathbf{e}$. A set of automorphisms, $\mathcal{B}(\oplus)$, is balanced if $\mathbf{e} \in \mathcal{B}(\oplus)$ and if $f \in \mathcal{B}(\oplus)$ implies $-f \in \mathcal{B}(\oplus)$.

Lemma 1. Let $V(\oplus)$ an abelian group with identity e and $\mathcal{B}(\oplus) \subset$ Aut $V(\oplus)$ a balanced set. Let $v \in V$ and $f \in \mathcal{B}(\oplus)$ arbitrary. Suppose for every $g \neq-f$, in $\mathcal{B}(\oplus)$, there exists $u \in V$ such that $(f \oplus g)(u)=v$. Then, the addition of automorphisms is closed on $\mathcal{B}(\oplus)$. Moreover, $\mathcal{B}(\oplus)$ is an abelian group with identity $\boldsymbol{e}$.

Proof. This result is telling us an easy way of knowing if $\mathcal{B}(\oplus)$ is a group with addition of functions. We of course require that $\mathcal{B}(\oplus)$ be balanced. Under addition of automorphisms, the inverse of $f$ is the function $-f$ that acts by $x \mapsto-(f(x))$. The inverse of $\mathbf{i d}_{V}$ is $-\mathbf{i d}_{V}$ that makes $x \mapsto-x$. Associativity in $V(\oplus)$ implies associativity in $\mathcal{B}(\oplus)$. The commutative property in $\mathcal{B}(\oplus)$ also follows from the commutative property in $V(\oplus)$.

Let us show $\mathcal{B}(\oplus)$ is closed under addition; $f, g \in \mathcal{B}(\oplus)$ implies $f \oplus g \in \mathcal{B}(\oplus)$. First we show $f \oplus g$ is a homomorphism of groups, then we show it is bijective. We start by verifying the relation of morphisms. Notice, we use commutativity of $V(\oplus)$ to prove this is true.

$$
\begin{aligned}
(f \oplus g)(x \oplus y) & =f(x \oplus y) \oplus g(x \oplus y) \\
& =f(x) \oplus f(y) \oplus g(x) \oplus g(y) \\
& =f(x) \oplus g(x) \oplus f(y) \oplus g(y) \\
& =(f \oplus g)(x) \oplus(f \oplus g)(y)
\end{aligned}
$$

To prove $f \oplus g$ is bijective, first we prove it is either injective or it is the trivial function $e$. Let $x, y \in V$ two distinct objects and suppose $(f \oplus g)(x)=(f \oplus g)(y)$. This simply means $f(x) \oplus g(x)=f(y) \oplus g(y)$. Use commutativity to find $e=f(x)-f(y)+g(x)-g(y)$. Using the fact that $f, g$ are automorphisms, we have $f(x-y)+g(x-y)=e$. Then we have $(f \oplus g)(x-y)=e$, because of the definition of $f \oplus g$. We will prove $f \oplus g=\mathbf{e}$. Take any object $a \neq e$, then $a=x-y$ for two non trivial objects $x,-y$; choose any non trivial object $x$ and then $-y$ is determined. This implies $(f \oplus g)(a)=e$. This proves the addition of two objects in $\mathcal{B}(\oplus)$ is an injective function, or it is the trivial function. From our hypothesis, we can immediately conclude the addition, $f \oplus g$, is a function onto $V$. Take any object $v \in V$. Then there exists $u \in V$ such that $(f \oplus g)(u)=v$.

Theorem 4. Let $V(\oplus)$ an abelian group and suppose $\mathcal{B}(\circ) \subset$ Aut $V(\oplus)$ is a balanced, closed and commutative set of automorphisms with composition. Suppose $\mathcal{B}(\oplus)$ satisfies the conditions of the Lemma. Then $\mathcal{B}(\oplus, \circ)$ is a field, and we refer to it as the field of automorphisms. We say $V$ is a linear space over the field of automorphisms $\mathcal{B}$. The elements of $V$ are called vectors.

Proof. With respect to composition, it is sufficient to verify $\mathcal{B}(\circ)$ is balanced, closed and abelian. This follows from the third part of Theorem 3 . If the conditions of the Lemma hold for $\mathcal{B}(\oplus)$, under addition of automorphisms, we know $\mathcal{B}(\oplus)$ is a group. Now we have to show the distributive property holds. This is the simple statement that $\circ f$ is a homomorphism on $\mathcal{B}(\oplus)$; this is expressed by $f \circ(g \oplus h)=(f \circ g) \oplus(f \circ h)$. 


$$
\begin{aligned}
(f \circ(g \oplus h))(x) & =f(g(x) \oplus h(x)) \\
& =f(g(x)) \oplus f(h(x)) \\
& =(f \circ g)(x) \oplus(f \circ h)(x) \\
& =((f \circ g) \oplus(f \circ h))(x)
\end{aligned}
$$

This proves $\mathcal{B}(\oplus, \circ)$ is a field. Now we shall prove we have the structure of a linear space, in the classic sense. The scalar product is simply the application of an automorphism to a vector. Let $f \in \mathcal{B}$, then the scalar product of $f$, with a vector $v \in V$, is defined as $f \cdot v=f(v)$. First, $(f \cdot g) \cdot v=(f \circ g)(v)=f(g(v))=f \cdot(g \cdot v)$ because $\circ$ is the product of the field. Also, $f \cdot(u \oplus v)=(f \cdot u) \oplus(f \cdot v)$ because $f \in$ Aut $V(\oplus)$. By definition of addition of functions, $(f \oplus g) \cdot v=(f \cdot v) \oplus(g \cdot v)$. This result is telling us is that a linear space is defined by an abelian group $V$ and a set of automorphisms (of $V$ ) that form a field.

Theorem 5. Given a field $K(+, \cdot)$, the additive group $K(+)$ is a linear space over $K(+, \cdot)$.

Proof. This classic result is easily proven in this context. This statement means that a field is a field of automorphisms for its additive group. The operation functions of the multiplicative group $K(\cdot)$ are automorphisms of $K(+)$, and they form a field. Said differently, the multiplicative group of a field is isomorphic to a field of automorphisms on the additive group. Let us use Theorem 4 to verify this is true. Theorem 3 implies the set of automorphisms, $\{\cdot x\}_{x \in K}$, forms a group under composition and this group is isomorphic to $K(\cdot)$ so that it is abelian. This covers the first part of the hypothesis in Theorem 4.

For the additive operation we must verify the conditions of the Lemma. Let $\cdot(x)$ an operation function of $K(\cdot)$. Then the additive inverse $-x$, of $x$, is an object in $K(\cdot)$. This implies $\cdot(-x)$ is an operation function of $K(\cdot)$. This proves $K(\cdot)$ is balanced. To prove it is closed, let $v \in K(\cdot)$ arbitrary. Because of the Lemma, it is sufficient to prove there exists $u \in K(\cdot)$ such that $((\cdot a) \oplus(\cdot b))(u)=v$. It is trivial to verify $u=v \cdot(a \oplus b)^{-1}$, where $(a+b)^{-1}$ is the multiplicative inverse element of $a+b$.

$$
\begin{aligned}
(\cdot(a) \oplus \cdot(b))\left(v \cdot(a+b)^{-1}\right) & =\cdot(a)\left(v \cdot(a+b)^{-1}\right) \oplus \cdot(b)\left(v \cdot(a+b)^{-1}\right) \\
& =\left(a \cdot v \cdot(a+b)^{-1}\right) \oplus\left(b \cdot v \cdot(a+b)^{-1}\right) \\
& =v \cdot\left[\left(a \cdot(a+b)^{-1}\right) \oplus\left(b \cdot(a+b)^{-1}\right)\right] \\
& \left.=v \cdot\left[(a+b) \cdot(a+b)^{-1}\right)\right] \\
& =v .
\end{aligned}
$$

\section{Finite Sets and Natural Numbers}

Finding a mathematical collection of objects that behave under rules that we can interpret as the order and operation of addition for natural numbers, is not an easy task. This problem was taken up by many mathematicians at the begining of the last century. When we talk about spacial geometry we understand we are referring to objects called points, lines and planes. In general we talk of collections of points, such as circles, or others. Just in the same way, if we wish to formalize the theories of arithmetic and anlaysis, we have to know what objects we are dealing with. The solution was found that we can formulate the statements of arithmetic, and later analysis, using an elementary concept, set. Attempts were then made to find set representations of numbers and to model the structure that we understand by $\mathbb{N}$, using sets. 
Being an elementary concept, we can not describe a set in terms of other mathematical objects. Rather, we describe all mathematical objects using the language of sets. So, we can only define a set, linguistically, as a collection of objects. But, it has been found that a definition this ambiguous, leads to more problems than it solves. So, the trick is to find a collection of sets that is well enough defined to serve our purposes, and that does not lead to conceptual paradoxes that have been pointed out in the literature. The problem is that considering arbitrary collections we can have strange sets such as $\{\{\{\cdots\{\{\cdots\}\} \cdots\}\}\}$ and other collections we do not need for the construction of $\mathbb{N}$. Although it has been found that we can incorporate such sets to an appropriate set theory, here we will take a different view. We will try to find the simplest possible representation of natural numbers and real numbers as sets. The two most widely used models of mathematics begin by describing the natural numbers as Hereditarily Finite Sets. This collection of sets, which we denote HFS, consists of the sets obtained in the following procedure. We say the set with no objects, $\emptyset$, is in HFS. Also, if $x_{1}, x_{2}, \ldots, x_{n}$ are objects in HFS, then the collection of these, $\left\{x_{1}, x_{2}, \ldots, x_{n}\right\}$ is also in HFS. When we wish to make the statement, that an object $x$ is in a set $X$ we denote this with $x \in X$. Let us construct sets using these parameters. We immediately know the collection $\{\emptyset\}$ is an object in HFS. Now that we have $\emptyset$ and $\{\emptyset\}$ in HFS, we know that the collection of these two objects, $\{\emptyset,\{\emptyset\}\}$ is also in HFS. Then, we can take $\emptyset$ and $\{\emptyset,\{\emptyset\}\}$ to find $\{\emptyset,\{\emptyset,\{\emptyset\}\}\} \in$ HFS. We can also use the sets $\{\emptyset\}$ and $\{\emptyset,\{\emptyset\}\}$ to find $\{\{\emptyset\},\{\emptyset,\{\emptyset\}\}\} \in$ HFS, etc. The first difficulty we have is ordering these sets so that we can model the order of natural numbers.

The solution Zermelo and Fraenkel found is to order a sub collection of HFS. Notice it is trivial to order the sets $\emptyset$, $\{\emptyset\}$, $\{\{\emptyset\}\},\{\{\{\emptyset\}\}, \ldots$. We can intuitively say that these sets are ordered by contention. If we only consider these sets, we have the order of natural numbers, $\mathbb{N}_{<}=\{\emptyset,\{\emptyset\},\{\{\emptyset\}\},\{\{\{\emptyset\}\}\}, \ldots\}$. Then we have to find a way of defining addition of these sets, in such a way that it serves as a model of addition of natural numbers. This simply means, we have to find an operation on these sets, that is commutative, associative and has an identity element. Proving these statements is usually tedious and laborious. But, the real difficulty arises in understanding the constructions and objects used to describe more complicated structures such as the integer numbers, rational numbers, and real numbers. These have to be built in terms of each other. Integers are described in terms of natural numbers. Rational numbers are described in terms of integers, and real numbers are defined in terms of rational numbers. The last step, in building real numbers, gives objects that are difficult to describe and work with, leading to a gap in most undergraduate students' learning since most programs do not include these constructions. Even modern day efforts to describe the real number system do not provide an easy way to understand the nature of the object we call real number. The second approach taken in describing the order of natural numers is due to Von Neumann. He begins by ordering the sets $\emptyset<\{\emptyset\}<\{\emptyset,\{\emptyset\}\}<\{\emptyset,\{\emptyset\},\{\emptyset,\{\emptyset\}\}\}<\{\emptyset,\{\emptyset\},\{\emptyset,\{\emptyset\}\},\{\emptyset,\{\emptyset\},\{\emptyset,\{\emptyset\}\}\}$. Here, we say that one of these sets, $x$, is smaller than an other, $y$, if $x \in y$. Of course, we can easily verify this order is transitive and anti symmetric. This approach has some advantages in simplifying some proofs for the order and addition of natural numbers. However, when building the later numerical structures, we have a similar situation as in the Zermelo-Fraenkel theory. The greater difficulty arises in building the real numbers. These constructions and their technical aspects can be consulted in [Bernays(1991)]

The fact that we have at least two different constructions, gave way to another question. This is formally referred to as Benaceraff's Identification Problem. It has a great deal to do more with the Philosophy of Mathematics, than the mathematical models in use, but it still has wide implications. The main statement is set forth in a publication titled "What Numbers are Not", [Benacerraf(1965)]. The argument is made that numbers are actually not sets because there is no absolute way of describing them in terms of sets. In fact, numbers do not exist at all. We are simply taking abstract objects, and giving them properties we want them to satisfy. But, there is no entities satisfying these properties. We make it all up. For example, we can not know what object the number 3 is. Zermelo-Fraenkel tell us $3=\{\{\{\emptyset\}\}\}$, but Von Neumann tells us $3=\{\emptyset$, $\{\emptyset\},\{\emptyset,\{\emptyset\}\}\}$. Who are we to believe? One school of thought says nobody. Here we will try to propose a canonical set theory, showing that numbers do exist and we can specifically say what object each one is. We will not only do this for natural numbers, we will also do this for real numbers, and this will lead to a theory of types we briefly discuss in the conclusions for later work. To do this, we take the approach of defining natural numbers as objects in HFS, also. However, the main difference is that we order all of the sets in HFS. Thus proving $\mathbb{N}=$ HFS. 
An interesting thing happens. The sets used in Z-F and VN are sub orders of our construction of $\mathbb{N}$. Let us be precise with this. The Z-F set theory assigns the numbers $0=\emptyset, 1=\{\emptyset\}, 2=\{\{\emptyset\}\}, 3=\{\{\{\emptyset\}\}\}, 4=\{\{\{\{\emptyset\}\}\}\}$, etc. Our construction of natural numbers assigns the Z-F objects to the numbers $0=\{\emptyset\}, 1=\{\emptyset\}, 2=\{\{\emptyset\}\}, 4=\{\{\{\emptyset\}\}\}, 16=\{\{\{\{\emptyset\}\}\}\}, 2^{16}=\{\{\{\{\{\emptyset\}\}\}\}\}, \ldots$. Thus, we can say Z-F Fraenkel ordinals are the sub order $\left\{0,1,2,2^{2}, 2^{2^{2}}, 2^{2^{2^{2}}}, \ldots, 2^{2^{2^{2}}}, \ldots\right\} \subset \mathbb{N}$. On the other hand, VN set theory assigns $0=\emptyset, 1=\{\emptyset\}, 2=\{\emptyset,\{\emptyset\}\}$, etc. We assign the VN objects to the numbers $2^{0}-1,2^{1}-1,2^{2}-1,2^{3}-1,2^{4}-1, \ldots$ The VN ordinals are the sub order $\left\{0,1,3,7,9,15, \ldots, 2^{n}-1, \ldots\right\} \subset \mathbb{N}$. Each of these constructions, Z-F and VN, order strict sub sets of $\mathbb{N}$. In this section we construct the structure of natural numbers using an order and addition operation on the set of all hereditarily finite sets. Our order and operation will be defined simultaneously in constructive manner.

\subsection{Motivation}

There is an intuitive motivation behind the axiomatic base given in this section. We discuss this now, to help understand the structure of order and addition of natural numbers, that we will be using. When adding numbers in base 10 (or base $b>2$ ) we have to use sequences of digits to represent natural numbers. We must specify how many times we consider each power of $b$. But, with binary representation we use a more elementary language. It suffices to specify if the power is considered or not, $\in, \notin$. This means that natural number is determined by a set of smaller natural numbers, that are those that appear as power in binary form. For example, the number $7=2^{0}+2^{1}+2^{2}$ is determined by the set $\{0,1,2\}$. This is commonly referred to as Ackermann Coding or BIT-predicate. This is an important part of the practical aspects this work has, since we are able to model mathematical systems directly in terms of classic computational processes. The ackermann coding does not give a means for adding numbers in any special manner. We have the same means of operating. Namely, carry over algorithms. This happens because when we add numbers we treat them as a sequence.

Here, we define addition treating natural numbers as sets. Let us consider the following addition of numbers $7+13=$ $\left(2^{0}+2^{1}+2^{2}\right)+\left(2^{0}+2^{2}+2^{3}\right)$. We are going to consider two new sets, the powers that are not repeated $\left(2^{1}+2^{3}\right)$, and the powers that repeat $\left(2^{0}+2^{2}\right)$. In terms of sets, we are considering the symmetric difference $\{1,3\}$ and the intersection $\{0,2\}$. Here we make the following observation. To add a power of 2 with itself we simply add 1 to the power. So, we can consider the sum $7+19=\left(2^{1}+2^{3}\right)+\left(2^{0+1}+2^{2+1}\right)=\left(2^{1}+2^{3}\right)+\left(2^{1}+2^{3}\right)$. Iterate the process, so we have to add 1 to the repeated powers. This gives $7+9=2^{1+1}+2^{3+1}=2^{2}+2^{4}=20$. If $A, B$ are two finite sets of natural numbers, we can add them using this method. Form two new sets $A^{\prime}=A \triangle B$ and $B^{\prime}=s(A \cap B)$. Then we have $A \oplus B=A^{\prime} \oplus B^{\prime}$. But, this alone does not get us anywhere. We have reduced the sum of two sets, $A \oplus B$, to the sum of two new sets $A^{\prime} \oplus B^{\prime}$. The sum $A^{\prime} \oplus B^{\prime}$ is in turn reduced to a sum $A^{\prime \prime} \oplus B^{\prime \prime}$, etc. So basically, we have done nothing. However, this is not the case, since we can guarantee that in a finite number of iterations the intersection $B^{(n)}=A^{(n-1)} \cap B^{(n-1)}=\emptyset$ becomes the empty set. This yields our final answer $A^{(n)}$ since our base case is $A \oplus \emptyset=\emptyset \oplus A=A$. Let us apply this reasoning with another example, $15+23=38$, from Figure 1 . We have the addition $A \oplus B=\{0,1,2,3\} \oplus\{0,1,2,4\}$ because $15=2^{0}+2^{1}+2^{+} 2^{3}$ and $23=2^{0}+2^{1}+2^{2}+2^{4}$. We find that $A^{\prime}=A \triangle B=\{3,4\}$ and $A \cap B=\{0,1,2\}$, so that $B^{\prime}=\{0+1,1+1,2+1\}=\{1,2,3\}$. We iterate the process with $A^{\prime \prime}=A^{\prime} \triangle B^{\prime}=\{1,2,4\}$ and $B^{\prime \prime}=A \cap B=\{3+1\}=\{4\}$. We can view this process as a Finite State Machine. A state is composed of two columns, each column is a finite configuration of energy-levels. A particle in the basic level is 1 unit, a particle in level 1 is worth 2 units. A particle in level 2 represents four units, etc. A finite configuration of particles in a column is representative of a set number in the obvious way so that each state is a pair of natural numbers. The initial state $t_{0}$ is given by the initial summands $A, B$. The next state, $t_{1}$ is again given by two columns. The configuration of the left column, is given by the energy levels that were not repeated in state $t_{0}$. The right column in $t_{1}$ is given by the objects that do repeat, but we displace these one level up. The configuration of state $t_{2}$ is defined similarly in terms of state $t_{1}$. The left column of state $t_{2}$ is given by the energy levels not repeated in state $t_{1}$. The configuration in the right column of state $t_{2}$ is given by the energy levels repeated in state $t_{1}$, but one level up. In a finite number of steps we reach a stable state with no occupation in the right column, giving us our result in the left column. It will not be difficult for the reader to prove the number of steps to reach stability is bounded above by $\max (A \cup B)$. For example, it takes at most $8=\max (\{0,1,2,3,5,6,8\})$ states to find $\{0,3,6\} \oplus\{0,1,2,5,8\}$. 


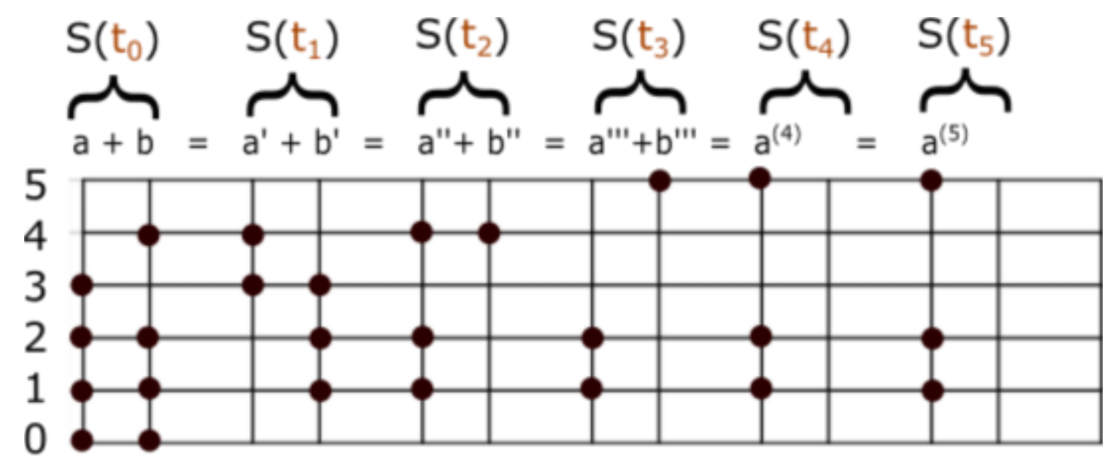

Figure 1: Graphic representation of $15+23=38$.

We will provide an addition operation for finite sets, and this operation is isomorphic to $\mathbb{N}_{+}$. The sum of two sets is expressed as the sum of two new sets, in a process that ends in finite steps. We take the view that an operation is a function whose domain is a space of functions itself; * :A $A(A f A)$ where $A f A$ is the set of all functions $A \rightarrow A$. This way, the operation $\oplus$ of sets is defined in terms of its functions $\oplus n$. Each function makes $\oplus n(x)=n \oplus x$. We begin by defining the function $\oplus 1$ which not only generates the hereditarily finite sets, it also generates the set of objects $\oplus n$. The family of functions $\oplus n$ is generated by a single function $\oplus 1$, all others being powers of composition, $\oplus 2=\oplus 1 \circ \oplus 1, \oplus 3=\oplus 1 \circ \oplus 1 \circ \oplus 1$, etc. In the forthcoming, consider the usual symmetric difference of sets, $A \triangle B=(A \cup B) /(A \cap B)$, and the partition $A \cup B=(A \triangle B) \cup(A \cap B)$. We define two base cases $0=\emptyset$ and $1=\{\emptyset\}$, along with a recursive function $\oplus 1:$ HFS $\rightarrow$ HFS defined by

$$
\oplus 1(A)=(A \triangle 1) \oplus s(A \cap 1)
$$

where $s:$ HFS $\rightarrow$ HFS sends every set $X=\{x\}_{x \in X}$ to the set $s(X)=\{\oplus 1(x)\}_{x \in X}$. In the following calculations we use the fact that $s(\emptyset)=\emptyset$. Furthermore, we define $A \oplus \emptyset=\emptyset \oplus A=\emptyset$ which simply defines $\emptyset$ as the identity element.

First we have $\oplus 1(0)=(0 \triangle 1) \oplus s(0 \cap 1)=1 \oplus s(\emptyset)=1 \oplus \emptyset=1$. The function $\oplus 1$ generates every element of HFS when applied successively.

$$
\begin{aligned}
2 & =\oplus 1(1)=(1 \triangle 1) \oplus s(1 \cap 1)=\emptyset \oplus s(1)=s(1)=\{\oplus 1(0)\}=\{1\} \\
3 & =\oplus 1(2)=(2 \triangle 1) \oplus s(2 \cap 1)=(\{1\} \triangle\{0\}) \oplus s(\{1\} \cap\{0\})=\{0,1\} \oplus s(\emptyset) \\
& =\{0,1\} \oplus \emptyset=\{0,1\} \\
4 & =\oplus 1(3)=(3 \triangle 1) \oplus s(3 \cap 1)=\{1\} \oplus s(\{0\})=2 \oplus\{\oplus 1(0)\}=2 \oplus\{1\} \\
& =2 \oplus 2
\end{aligned}
$$

Here we come upon a new object. We must find a suitable definition for $2 \oplus 2$, and in general we will need to find a suitable definition for $A \oplus B$. We simply extend our definition in the obvious way,

$$
A \oplus B=(A \triangle B) \oplus s(A \cap B)
$$

This gives

$$
2 \oplus 2=(2 \triangle 2) \oplus s(2 \cap 2)=\emptyset \oplus s(2)=\{\oplus 1(1)\}=\{2\} .
$$


Therefore,

$$
4=\{2\}
$$

This simply means the set $\{2\}=\{\{1\}\}=\{\{\{\emptyset\}\}\}$ is the object we know as the number 4 . We continue to generate sets, by applying the function $\oplus 1$ to the result.

$$
\begin{aligned}
& 5=\oplus 1(4)=(4 \Delta 1) \oplus s(4 \cap 1)=\{0,2\} \oplus s(\emptyset)=\{0,2\} \\
& 6=\oplus 1(5)=(5 \Delta 1) \oplus s(5 \cap 1)=\{2\} \oplus s(\{0\})=\{2\} \oplus\{\oplus 1(0)\}=\{2\} \oplus\{1\} .
\end{aligned}
$$

The set $\{\emptyset,\{\{\emptyset\}\}\}$ is the number 5 . To find the set that is the number 6 , we use the definition of addition of two sets to find $\{2\} \oplus\{1\}=\{1,2\} \oplus s(\emptyset)=\{1,2\}=\{\{\emptyset\},\{\{\emptyset\}\}\}$. This means $6=\{1,2\}=\{\{\emptyset\},\{\{\emptyset\}\}\}$. We notice, first of all, that the sum of two disjoint sets is the union, and secondly that every natural number is a set of smaller natural numbers. When we refer to hereditarily finite sets, in this manner, we call them set numbers because we give them the structure of natural numbers. Let $N$ be a natural number with binary representation $\sum_{i=1}^{n} 2^{a_{i}}$, then $N$ is the set number $\left\{a_{1}, a_{2}, \ldots, a_{n}\right\}$. For example, $5=\{0,2\}$ because $6=2^{0}+2^{2}$, while $6=\{1,2\}$ because $6=2^{1}+2^{2}$. We can easily find $11=\{0,1,3\}$.

$$
11=5 \oplus 6=\{0,2\} \oplus\{1,2\}=\{0,1\} \oplus s(\{2\})=\{0,1\} \oplus\{3\}=\{0,1,3\} .
$$

We can find $7=\{0\} \oplus\{1,2\}=\{0,1,2\}$ and then

$$
11=7 \oplus 4=\{0,1,2\} \oplus\{2\}=\{0,1\} \oplus s(\{2\})=\{0,1,3\} .
$$

The fact that the binary representation is involved is not a coincidence. Let us look at this from another point of view, and consider the number 13. We wish to find its natural representation so we start with its binary elements $13=\{0,2,3\}$. Then, $2=\{1\}$ and $3=\{0,1\}$. But, $1=\{0\}$ so that we only need to assign $0=\emptyset$ as the base case. We finally get $13=\{\emptyset,\{\{\emptyset\}\},\{\emptyset,\{\emptyset\}\}\}$ which is the same we obtain from $(11 \oplus 1) \oplus 1$. We give an adequate axiomatic base for proving thee statements. We construct mathematical sets, using heredeitarily finite sets and the axioms we need are stated in the following subsection.

\subsection{Axiomatic Base}

We begin with the empty set and the set that contains that set, $0=\emptyset$ and $1=\{\emptyset\}$. Notice that using our definition of addition of sets allows to build hereditarily finite sets. But, if we simply used union and intersection, we can not generate more sets beyond 0,1. So, our definition $A \oplus B=(A \triangle B) \oplus s(A \cap B)$ allows us to lift off the ground and generate new sets. We will have an axiom for the following statement. If $x$ is a set, then the object $\oplus 1(x)$ is a set. If this axiom should be true we first need for the union and intersection of sets to be sets, because the function $\oplus 1$ is defined in terms of union and intersection of sets. This raises the following question. Why are we able to generate new sets, using $\oplus 1$, if it is defined in terms of $\cap, \cup$, which do not generate new sets? The reason is because $\oplus 1$ is also defined in terms of itself when we say $\oplus 1(0)=1$ and $\oplus 1(1)=\{\oplus 1(0)\}=\{1\}$. Adding an object to the elements of a set is what allows us to find $2,3,4, \ldots$. 
We give the axiomatic base for this model of $\mathbb{N}$. We assume the existence of the empty set $0=\emptyset$ and the set that contains $0,1=\{\emptyset\}$. We also assume the union of sets and the intersection of sets, is a set. We will also assume any sub collection of a set, is a set. This will be the first part of our axioms. Our second axiom is the statement that $\oplus 1$ sends sets to sets. This axiom states that every set in HFS is obtained by applying the function $\oplus 1$ sufficiently many times. In other words, it simply states that no matter how many times we apply $\oplus 1$, we will always get a new object in HFS. These new objects we obtain from the function, are called sets and we find them in linear order. Once we have this, we use the union axiom to find $\mathbb{N}=\bigcup_{n} \oplus 1^{n}(0)$ is a set. It is the set that contains all the objects generated by $\oplus 1$ and 0,1 . This is the set of natural numbers $\mathbb{N}=$ HFS. In this section we provide the axioms needed for the formal construction of $\mathbb{N}$. In a later section we will see that real numbers are infinite sets of natural numbers, and we will provide an additional axiom in order to construct larger sets.

Axiom 1. The empty collection $\emptyset$ is a set, as is the collection $\{\emptyset\}$. If $A, B$ are two sets then $A \cup B$ is a set, and $A \cap B$ is a set. If $A$ is a set, then any sub collection $X \subset A$ is also a set.

With this first axiom, we are not able to go beyond the sets $\emptyset$ and $\{\emptyset\}$. The following definition and axiom will allow us to build all hereditarily finite sets, in linear order.

Definition 7. Define the set operation $\oplus$ with $A \oplus B=(A \triangle B) \oplus\{x \oplus 1\}_{x \in A \cap B}$.

To make our definition good, we set $\oplus 0(x)=x$. We have seen in the last sub section, how to find $\oplus 1(1), \oplus 1(2), \ldots$. When carrying out the calculations for $3 \oplus 1$ we recognized that it was necessary to know the value of $\oplus 2$ (2). If we continue to apply $\oplus 1$, we encounter more calculations of the form $\oplus x(y)$. But, our operation function for $\oplus x$ is explicitly dependent of $\oplus 1$. This is an interesting situation. The functions $\oplus x$ are defined as powers of $\oplus 1$, but to find $\oplus 1$ we also need to start finding $\oplus x$. The reason for this is that the operation functions build each other simultaneously, as we have seen in the calculations above.

Axiom 2. The operation function $\oplus 1$ generates all $\mathbf{H F S}$ when applied successively to 0 . The order in which sets are generated is an order of $\boldsymbol{H F S}$, equivalent to the order of natural numbers $\mathbb{N}_{\leq}$.

This axiom states that every set in HFS is obtained by applying the function $\oplus 1$ successively to 0 . That is to say, every hereditarily finite set is of the form $(\oplus 1 \circ \oplus 1 \circ \cdots \circ \oplus 1)(0)=1 \oplus(1 \oplus(1 \oplus \cdots(1 \oplus 0)))$. Furthermore, our construction of finite sets is at the same time providing an order because we construct the finite sets in order,

$$
0 \rightarrow_{\oplus 1} 1 \rightarrow_{\oplus 1} 2 \rightarrow_{\oplus 1} 3 \rightarrow_{\oplus 1} 4 \rightarrow_{\oplus 1} \cdots
$$

That is to say, hereditarily finite sets are ordered in terms of the order of construction. The order in which we find the elements of HFS is the natural order given to these. An important difference between this construction, and the Von-Neumann Ordinals and the Zermelo-Fraenkel Ordinals is that we order all HFS, while the latter two order transitive subsets of HFS. We have given an order and operation on HFS, that are isomorphic to $\mathbb{N}(\leq)$ and $\mathbb{N}(+)$, respectively. The commutative property of $\oplus$ is trivial because symmetric difference and intersection are commutative.

$$
\begin{aligned}
A \oplus B & =(A \triangle B) \oplus s(A \cap B) \\
& =(B \triangle A) \oplus s(B \cap A) \\
& =B \oplus A
\end{aligned}
$$

The easiest way to prove associative property of set sum is to prove the functions $\oplus x$ and $\bar{\oplus} y$ commute, for every set numbers $x, y$. Given that commutativty holds, we know $\oplus y=\bar{\oplus} y$. It is sufficient to prove the commutative property holds for operation functions, $\oplus x \circ \oplus y=\oplus y \circ \oplus x$, because of Proposition 3 
Proposition 4. The associative property holds for $\oplus$.

Proof. Let $a \in \mathbb{N}$, our second axiom states that to add $n$ we must apply $\oplus 1$ a total of $n$ times, $\oplus n(a)=\oplus 1^{n}(a)$. This proves the operation functions $\oplus m, \oplus n$ commute,

$$
\begin{aligned}
(\oplus n \circ \oplus m)(a) & =\oplus n(\oplus m(a)) \\
& =\oplus 1^{n}\left(\oplus 1^{m}(a)\right) \\
& =\oplus 1^{m}\left(\oplus 1^{n}(a)\right) \\
& =\oplus m(\oplus n(a)) \\
& =(\oplus m \circ \oplus n)(a) .
\end{aligned}
$$

We are simply using the fact that $f^{n} \circ f^{m}=f^{m} \circ f^{n}$ for any bijection $f$.

Now we give the following result which provides a practical way of defining the natural order of HFS, and which will allow us to define our order of finite groups, among other applications such as real analysis which we will see briefly at the end. Take two distinct natural numbers $A, B$ and consider their symmetric difference $A \triangle B$ which is not empty and is bounded. That is to say, $\max (A \triangle B)$ exists. Furthermore, this maximum is in exactly one of the two sets, not in both. We compare two sets in terms of this object, $\max (A \triangle B)$. The set that contains this object is the largest of the two. For example, $15=\{0,1,2,3\}<\{4\}=16$ because $A \triangle B=\{0,1,2,3,4\}$ and the maximum of this set is in $16=\{4\}$.

Theorem 6. If $A, B$ are two set numbers, then $A<B$ if and only if $\max (A \triangle B) \in B$.

Proof. Let $A=\left\{a_{1}, a_{2}, \ldots, a_{n}\right\}$ be a set number, and suppose $B$ is a set number such that $A<B$. From the second axiom we know that the set number $B$ is obtained by successively adding 1 to the set number $A$. This means $B=\oplus 1^{n}(A)$ for some $n \in \mathbb{N}$. We shall prove $\max (A \triangle B) \in B$ for every $B>A$. In this proof we will use the fact that $A \oplus B=A \cup B$ if $A \cap B=\emptyset$; this is a direct consequence of the addition of the definition of addition for set numbers. We start with $A \oplus 1=\left\{a_{1}, a_{2}, \ldots, a_{n}\right\} \oplus\{0\}$. There are two possibilities; $0 \notin A$ or $0 \in A$. In the first case we are done since $A \oplus 1=\left\{0, a_{1}, a_{2}, \ldots a_{n}\right\}$. Suppose $0 \in A$, then we have $A \oplus 1=\left\{0, a_{2}, \ldots, a_{n}\right\} \oplus\{0\}=\left\{a_{2}, a_{3}, \ldots, a_{n}\right\} \oplus\{1\}$. We have two possibilities $1 \notin A$ or $1 \in A$. In the first case we are done and we have $A \oplus 1=\left\{1, a_{2}, a_{3}, \ldots, a_{n}\right\}$. In the second case we have $a_{2}=1$ and this implies $A \oplus 1=\left\{a_{3}, a_{4}, \ldots, a_{n}\right\} \oplus\{2\}$. We continue in this manner until we come upon the first natural number that is not in $A$. Let us suppose $k$ is the smallest number not in $A$. Then, we have $A=\left\{0,1, \ldots, k-1, a_{k+1}, a_{k+2}, \ldots, a_{n}\right\}$, where $k<a_{k+1}<a_{k+2}<\ldots<a_{n}$. Applying $\oplus 1$,

$$
A \oplus 1=\left\{k, a_{k+1}, a_{k+2}, \ldots, a_{n}\right\}
$$

Now we take the symmetric difference and find its maximum, $A \triangle(A \oplus 1)=\{0,1,2, \ldots, k-1, k\}$. Since $k \in A \oplus 1$, we are done proving $\max (A \triangle(A \oplus 1)) \in A \oplus 1$. If we apply $\oplus 1$ to our result, we get

$$
A \oplus 2=\left\{k, a_{k+1}, a_{k+2}, \ldots, a_{n}\right\} \oplus\{0\}=\left\{0, k, a_{k+1}, a_{k+2}, \ldots, a_{n}\right\}
$$

This means $A \triangle(A \oplus 2)=\{1,2, \ldots, k\}$ and again we have that the maximum is in $A \oplus 2$. We can add a unit again, to get $A \oplus 3=\left\{1, k, a_{k+1}, a_{k+2}, \ldots, a_{n}\right\}$ which gives us the symmetric difference $A \triangle(A \oplus 3)=\{0,2,3, \ldots, k\}$ with maximum in $A \oplus 3$. Then we have $A \oplus 4=\left\{0,1, k, a_{k+1}, a_{k+2}, \ldots, a_{n}\right\}$ and symmetric difference $A \triangle(A \oplus 4)=\{2,3,4, \ldots, k\}$. 
We continue in this manner, applying $\oplus 1$, until we have applied it $2^{k}-1$ times. Thus far, we have $\operatorname{proven} \max (A \triangle B) \in B$ if $A<B<A \oplus 2^{k}$. Now, if we apply $\oplus 1$ once more, we are simply adding the singleton $2^{k}=\{k\}$, to get $A \oplus\{k\}=$ $\left\{0,1, \ldots, k, a_{k+1}, a_{k+2}, \ldots, a_{n}\right\}$. We conclude $\max \left(A \triangle\left(A \oplus 2^{k}\right)\right)=\max \{k\}=k \in A \oplus 2^{k}$.

Now we fall into repetition of what we have done up to this point. When we apply $\oplus 1$ to $A \oplus\{k\}$, we are going to substitute all the elements $0,1, \ldots, k$ with $k \oplus 1$. So, we have two possible cases; $k \oplus 1 \notin A$ or $\oplus 1 \in A$. In the first case we are done proving $\max \left(A \triangle\left(\left(A \oplus 2^{k}\right) \oplus 1\right)\right) \in\left(A \oplus 2^{k}\right) \oplus 1$. In the second case we come to

$$
\left(A \oplus 2^{k}\right) \oplus 1=\left\{k \oplus 1, a_{k+2}, \ldots, a_{n}\right\} \oplus\{k \oplus 1\} .
$$

The result of this, again depending on two possible cases. We see what we have to do. When we added 1 to $A \oplus 2^{k}$, we have to find the smallest number that is not in this set. Let us suppose it is the number $p>k$. This means $k, p$ are the two smallest numbers not in $A$, so that $A=\left\{0,1, \ldots, k-1, k+1, k+2, \ldots, p-1, a_{n-p+1}, a_{n-p+2}, \ldots, a_{n}\right\}$. We will have the result $\left(A \oplus 2^{k}\right) \oplus 1=\left\{p, a_{n-p+1}, a_{n-p+2}, \ldots, a_{n}\right\}$. This gives the symmetric difference $\{0,1, \ldots, k-1, k+1, k+2, \ldots, p-1, p\}$. We have proven $\max (A \triangle B) \in B$ if $A<B \leq\left(A \oplus 2^{k}\right) \oplus 1$.

We move to prove this is true for $\left(A \oplus 2^{k}\right) \oplus 2=\left\{0, p, a_{n-p+1}, a_{n-p+2}, \ldots, a_{n}\right\}$; the symmetric difference with $A$ is the set $\{1,2, \ldots, k-1, k+1, k+2, \ldots, p-1, p\}$. We continue in this manner and we reach

$$
\left(A \oplus 2^{k}\right) \oplus 2^{k}=\left\{0,1, \ldots, k-1, p, a_{n-p+1}, a_{n-p+2}, \ldots, a_{n}\right\} .
$$

Then we have symmetric difference $A \triangle\left(\left(A \oplus 2^{k}\right) \oplus 2^{k}\right)=\{k+1, k+2, \ldots, p\}$. We keep adding 1 until we reach $\left(A \oplus 2^{k}\right) \oplus 2^{p}=$ $\left\{0,1, \ldots, q-1, a_{n-q+2}, a_{n-q+3}, \ldots, a_{n}\right\}$ where $A=\left\{0,1, \ldots, k-1, k+1, \ldots, p-1, p+1, \ldots, q-1, a_{n-q+2}, a_{n-q+3}, \ldots, a_{n}\right\}$ and $q>p$. We continue in this manner finding $k, p, q, \ldots$ until we reach the largest number $r \notin A$ such that $r<a_{n}$. This proves $\max (A \triangle B) \in B$ if $A<B<A \oplus 2^{k} \oplus 2^{p} \cdots \oplus 2^{r}$. When we add 1 to $A \oplus 2^{k} \oplus 2^{p} \oplus \cdots \oplus 2^{r}=\left\{0,1, \ldots, a_{n}\right\}$, the result is the singleton $\left\{a_{n}+1\right\}$. Now it is trivial that the maximum of the symmetric difference is in $A \oplus 2^{k} \oplus 2^{p} \cdots \oplus 2^{r} \oplus 1$, since $\max (A)<\max \left(A \oplus 2^{k} \oplus 2^{p} \cdots \oplus 2^{r} \oplus 1\right)$. Observe that adding 1 to $X$ leaves the maximum of $X$ equal, or larger by one. We conclude the result also holds for any $X>A \oplus 2^{k} \oplus 2^{p} \cdots \oplus 2^{r} \oplus 1$ because

$$
\max (X) \geq \max \left(A \oplus 2^{k} \oplus 2^{p} \cdots \oplus 2^{r} \oplus 1\right)>\max (A)
$$

implies $\max (A \triangle X) \in X$.

Proving the second implication is easy. We use the following observation from the first part of this proof. Given $A=$ $\left\{a_{1}, a_{2}, \ldots, a_{n}\right\}$ and any number $x \notin A$, we can find a number $N$ such that $A \oplus N=\left\{x, a_{i}, a_{j}, \ldots, a_{n}\right\}$; of course if $x>A$ we have $A \oplus N=\{x\}$. Suppose $M=\max (A \triangle B) \in B$, then we add 1 until we obtain the result $A \oplus N=\left\{M, a_{i}, a_{j}, \ldots, a_{n}\right\}$, where $a_{i}, a_{j}, \ldots, a_{n}$ are the elements of $A$ that are greater than $M$. If there are no elements of $A$ greater than $M$, we simply have $A \oplus N=\{M\}$. Now we need to add $P$ to $A \oplus N$, where $P=\left\{b_{1}, b_{2}, \ldots, b_{\alpha}\right\}$ is the set of objects in $B$ that are smaller than $M$. The result is

$$
A \oplus(N \oplus P)=(A \oplus N) \oplus P=B
$$

which implies $A<B$.

Let us carry out an example of finding which of two different set numbers is largest. Let $A=\{2,5,6,8,9\}$ and $B=$ $\{0,1,7,8,9\}$. The largest of the two is the set that contains $\max \{0,1,2,5,6,7\}=7$, so that $A<B$. In the next sections we will have to find the order of set numbers given in a different form. For example, we may write a set number in the form $A=\{\{3,5\},\{1,2\},\{4,6\}\}=2^{2^{2^{3}+2^{5}}+2^{2^{1}+2^{2}}+2^{2^{4}+2^{6}}}$. Let us compare it with $B=\{\{3,4\},\{1,2\},\{5,6\}\}=2^{2^{2^{3}+2^{4}}+2^{2^{1}+2^{2}}+2^{2^{5}+2^{6}}}$. Obviously $A<B$ since $\max (A)=\{4,6\}<\{5,6\}=\max (B)$. 


\subsection{Product of Set Numbers}

The product is easy to define, and we have already defined multiplication by 2 . In binary representation, we have $2^{n}+2^{n}=2^{n+1}$, so here we have a corresponding rule. If a power is repeated we add 1 to that power. Therefore, to multiply by 2 is to apply the function $\odot 2=s$ that adds 1 to the elements of the argument. Then, multiplication by 4 is $s \circ s$ which adds 2 to the elements of the argument. In general to multiply a set number $B$ by $2^{k}$, we apply the function $s^{k}$, to $B$. If $B=\left\{b_{1}, b_{2}, \ldots, b_{n}\right\}$ then $2^{k} \odot B=\left\{b_{1} \oplus k, b_{2} \oplus k, \ldots, b_{n} \oplus k\right\}$. Multiplying $B$ by $2^{k}$ is displacing all the objects of $B$, in our graphic representation, $k$ units up. We say $2^{k} \odot B$ is the $k$-displacement of $B$. We define the product $A \odot B$ in terms of displacements of the base $B$, and the pivot $A$.

$$
A \odot B=\bigoplus_{a \in A}\{b \oplus a\}_{b \in B}
$$

This means we add displacements of $B$, one for each object of the pivot $A$. If $a \in A$ then the $a$-displacement of $B$ is one of the displacements in our sum. We notice that multiplication by 0 results in the empty set, $0 \odot X=X \odot 0=0$. It is also trivial to find $1 \odot X=X \odot 1=X$. To show that $2=\{1\}$ is commutative under multiplication,

$$
\begin{aligned}
\{1\} \odot X & =\{x \oplus 1\}_{x \in X} \\
& =\bigcup_{x \in X}\{x \oplus 1\} \\
& =\bigoplus_{x \in X}\{1 \oplus x\} \\
& =X \odot\{1\} .
\end{aligned}
$$

This means $2 \odot X=X \odot 2=X \oplus X$. Before proving general properties, let us calculate $3 \odot 5=\{0,1\} \odot\{0,2\}$ in two different ways to verify these numbers commute. We first make $A=3$ and $B=5$ so that we will add two displacements of $B=\{0,2\}$. The first displacement is $\{0 \oplus 0,2 \oplus 0\}=\{0,2\}$, and our second displacement is $\{0 \oplus 1,2 \oplus 1\}=\{1,3\}$. We add the two and obtain $\{0,2\} \oplus\{1,3\}=\{0,1,2,3\}=15$. Now we take $A=5$ and $B=3$, so that we will add two displacements of $3=\{0,1\}$, each corresponding to an element of $5=\{0,2\}$. Our displacements of 3 are the 0 -displacement, $\{0 \oplus 0,1 \oplus 0\}=\{0,1\}$, and the 2-displacement, $\{0 \oplus 2,1 \oplus 2\}=\{2,3\}$. Adding these two displacements results in $\{0,2\} \oplus\{1,3\}=\{0,1,2,3\}=15$. Let us give an example in terms of powers of 2 , to illustrate this procedure. To find the product $\left(2^{0}+2^{1}\right)\left(2^{0}+2^{2}\right)$ we distribute $2^{0}\left(2^{0}+2^{2}\right)+2^{1}\left(2^{0}+2^{2}\right)$. Then, we have $\left(2^{0+0}+2^{2+0}\right)+\left(2^{0+1}+2^{2+1}\right)=\left(2^{0}+2^{2}\right)+\left(2^{1}+2^{3}\right)$. In Figure 2 we have the visual representation of $7 \odot 9$.

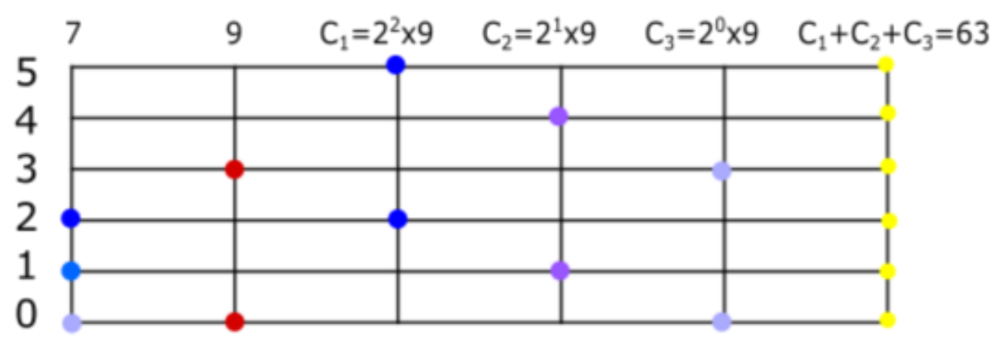

Figure 2: We find the product $7 \odot 9$. The first and second columns are the pivot and base, respectively. The next three columns correspond to the displacements of our base. The last column is the sum of the displacements. The result is equal to $63=\{0,1,2,3,4,5\}$. 
To formalize this, we first verify $\odot 2$ is a morphism for addition of set numbers, $s(A \oplus B)=s(A) \oplus s(B)$. We know $X \oplus X=s(X)$, so that $s(A \oplus B)=(A \oplus B) \oplus(A \oplus B)=(A \oplus A) \oplus(B \oplus B)=s(A) \oplus s(B)$. Of course this implies $s^{k}(A \oplus B)=s^{k}(A) \oplus s^{k}(B)$. To prove the distributive property in general form, we use the distributive property of powers of 2 , and the commutative and associative properties of addition of sets.

$$
\begin{aligned}
A \odot(B \oplus C) & =\bigoplus_{a \in A}\{x \oplus a\}_{x \in B \oplus C} \\
& =\bigoplus_{a \in A} s^{a}(B \oplus C) \\
& =\bigoplus_{a \in A}\left(s^{a}(B) \oplus s^{a}(C)\right) \\
& =\bigoplus_{a \in A} s^{a}(B) \oplus \bigoplus_{a \in A} s^{a}(C) \\
& =(A \odot B) \oplus(A \odot C)
\end{aligned}
$$

Now we prove multiplication is commutative. Let $a \in A$ fixed, then the set $\{b \oplus a\}_{b \in B}=\left\{b_{1} \oplus a, b_{2} \oplus a, \ldots, b_{n} \oplus a\right\}$ can be expressed as a sum of disjoint singletons, $\bigoplus_{b \in B}\{b \oplus a\}$

$$
\begin{aligned}
A \odot B & =\bigoplus_{a \in A}\{b \oplus a\}_{b \in B} \\
& =\bigoplus_{a \in A} \bigoplus_{b \in B}\{b \oplus a\} \\
& =\bigoplus_{b \in B} \bigoplus_{a \in A}\{a \oplus b\} \\
& =\bigoplus_{b \in B}\{a \oplus b\}_{a \in A} \\
& =B \odot A .
\end{aligned}
$$

We have proven the distributive and commutative properties, so that we have also proven

$$
(A \oplus B) \odot C=(A \odot C) \oplus(B \odot C) .
$$

Now, we wish to prove that multiplication is associative. We need the following proposition.

Proposition 5. The operation function $\odot N$ acts on sets by $\odot N(X)=\oplus X^{N}(0)$.

Proof. This is proven by mathematical induction. We know it is true for 1 , since $1 \odot X=X$. Suppose it is true for $N$, then using the distributive property of 4

$$
\begin{aligned}
\odot(N \oplus 1)(X) & =\odot N(X) \oplus \odot 1(X) \\
& =\oplus X^{N}(0) \oplus X \\
& =\oplus X\left(\oplus X^{N}(0)\right) \\
& =\oplus X^{N+1}(0) .
\end{aligned}
$$


Now we can prove the associative property holds for the product of set numbers. Because of Proposition 3 , it is sufficient to verify the operation functions of $\odot$ commute. We will use the notation $\bigoplus_{i=1}^{N} X$ to represent the number $\oplus X^{N}(0)$. This way, the expression $\bigoplus_{j=1}^{A}\left(\bigoplus_{i=1}^{B} X\right)$ means we add $X$ a number of $B$ times to obtain the number $B \odot X$, and then we add $B \odot X$ a total of $A$ times to obtain $A \odot(B \odot X)$. We are really adding $X$ a total number of $A \odot B=B \odot A$ times. It is as if we have a rectangular matrix of size $A \times B$ and every entry is equal to $X$, then we add all the entries. Considering the matrix of size $B \times A$ and proceeding is equivalent to rearranging the order of the sum. To prove associativity of product we apply Proposition 5 twice, then we use commutativity and associativity of addition to find the third equality. Then we apply Proposition 5 again.

$$
\begin{aligned}
(\odot A \circ \odot B)(X) & =\odot A\left(\bigoplus_{i=1}^{B} X\right) \\
& =\bigoplus_{j=1}^{A}\left(\bigoplus_{i=1}^{B} X\right) \\
& =\bigoplus_{i=1}^{B}\left(\bigoplus_{j=1}^{A} X\right) \\
& =\odot B\left(\bigoplus_{i=1}^{A} X\right) \\
& =(\odot B \circ \odot A)(X)
\end{aligned}
$$

In [I], we also give description of subtraction, division and powers of set numbers. Here, we do not mention them because they are not needed for the purposes of this work. In a later publication we will treat real numbers and operations with more detail than provided in [I].

\subsection{Integers}

We will not require the structure of integers, in order to construct the structure of real numbers. However, we provide a construction of $\mathbb{Z}$ because it introduces methods and concepts of previous and later sections. We will use operation functions and their inverse functions to describe integers. A positive integer $n \in \mathbb{Z}$ is an operation function $\oplus n$, while its negative integer $-n \in \mathbb{Z}$ is the inverse function $(\oplus n)^{-1}$. We notice one important fact. Negative integers can be distinguished from positive integers. The inverse function is of the form $-n:\{n, n \oplus 1, n \oplus 2, \ldots\} \rightarrow \mathbb{N}$, while a positive integer is a function of the form $\mathbb{N} \rightarrow\{n, n \oplus 1, n \oplus 2, \ldots\}$. This will have to be considered when defining addition of integers; it does not represent any difficulty but we are careful to satisfy the picky reader. The integer 0 is the identity function of $\mathbb{N}$. We represent the set of negative integers with the symbol $-\mathbb{N}$. We say $X \subset \mathbb{Z}$ is a non negative subset of $\mathbb{Z}$ if $-\mathbb{N} \cap X=\emptyset$, and the like. We will use the symbol $n=\oplus n$ and $-n$ to represent integers.

The sum of integers is defined in the obvious way, using composition. The sum of two positive integers $n, m$ is the composition and the sum of two negative integers is the negative of the composition, $(-n)+(-m)=-(n \circ m)$. We can do this because the composition of two positive integers is a positive integer. Also, the composition of two negative integers is defined and equal to a negative integer, strictly speaking. It is equal to the function $-n \circ-m=-(n \circ m):\{n \oplus m,(n \oplus m) \oplus 1,(n \oplus m) \oplus 2, \ldots\} \rightarrow \mathbb{N}$. The sum of one positive and one negative integer is defined as follows. Let $(-n), m$ be such numbers, then the function $(-n) \circ m$ exists. We can have two cases. If the corresponding natural numbers satisfy $n<m$, we know there is a natural 
number $x$ such that $m=n+x$. We define $-n+m=\oplus x$, and we have $\oplus x=-n \circ m: \mathbb{N} \rightarrow\{m-n, m-n+1, \ldots\}$. In the contrary case that the natural numbers satisfy $m<n$, we have $n=m+x$ for some natural number $x$. We define the addition of the integers as $-n+m=(\oplus x)^{-1}=-x$, and we have $-x=-n \circ m:\{n-m, n-m+1, \ldots\} \rightarrow \mathbb{N}$. In other words, the order relation between $n, m$ tells us if $-n+m$ is a positive integer $(n<m)$ or a negative integer $(m<n)$. But, what happens if we try to define $m+(-n)$ ? Consider the composition $m \circ-n$. It does not matter if $m<n$ or $n<m$. This function is defined only for numbers greater than or equal to $n$, and the elements in the image are greater than or equal to $m$, so that we have $m \circ(-n):\{n, n+1, \ldots\} \rightarrow\{m, m+1, \ldots\}$. Although $m \circ-n$ is a well defined composition, it is not an integer. The functions $m \circ(-n)$ and $-n \circ m$ are not the same function, but in the intersection of the domains they are equal functions. We are justified in defining the sum of integers as commutative. Associativity for addition of integers follows because it is defined in terms of composition of functions. Let us find the result of $5-3$, so we start with the function $-3 \circ 5$. Remember, -3 represents the function minus 3 , and 5 represents the function plus 5 . Since $5=3+2$, we have the function plus 2 as the result of $5-3$. Now, consider $5-7$ so that $7=5+2$. The result of this integer operation is the function minus 2 . Ordering integers is natural, in this context. Two integers $x, y$ satisfy the inequality $x<y$ if and only if $x(n)<y(n)$, for any $n \in \mathbb{N}$. For example, we know $-5<2$ because $-5(5)=0<7=2(5)$. Of course, the order is well defined so that there is no natural number $n$ such that $2(n)<-5(n)$. Let us prove $-6<-3$. We need a number that is in the domain of -3 and -6 , say 6 . Then, $-6(6)=0<3=-3(6)$.

\section{Finite Functions and Permutations}

In this section we will build the set of finite functions on $\mathbb{N}$, and provide an injective function from this set, into the set of natural numbers. The important quality of this representation is that functions are equivalent if and only if they are represented by the same number. We find a way to assign natural numbers to abstract functions as well. There will be a distinct difference when we are working with an abstract function or a concrete function. Functions with the same structure are those assigned the same natural number. This does not happen when we work with concrete functions on $\mathbb{N}$. Concrete functions with the same structure can have different numeric representation. For example, consider the functions $f, g$ defined by

$$
\begin{aligned}
& f(a)=b \quad g(a)=a \\
& f(b)=a \quad g(b)=c \\
& f(c)=c \quad g(c)=b
\end{aligned}
$$

These two abstract functions have the same structure, and have the same numeric representation; they will be considered to be the same function. However, if the objects are not abstract, $a, b, c \in \mathbb{N}$, then $f, g$ are concrete functions and they will be represented by distinct numbers. In our example, let $a=3, b=5$ and $c=0$. The functions $f, g$ defined by $f(3)=5, f(5)=3$, $f(0)=0$, and $g(3)=3, g(5)=0, g(0)=5$ are different and they will be represented by different numbers.

In this section we order the set of finite functions of $\mathbb{N}$. Then we give an equivalence definition for abstract finite functions, which simultaneously orders these equivalence classes. In the process we are also able to provide a canonical order for the elements of a given abstract finite function, and we can say which objects of the function can be considered to be equivalent. In our example, the objects $a, b$, are equivalent in the function $f$, while $c$ is not equivalent to another object. The objects $b, c$ are equivalent in the function $g$, and $a$ is not equivalent to another object. 


\subsection{Ordered Pairs}

If we wish to represent finite functions as natural numbers, we will first find a way of representing ordered pairs as natural numbers. An ordered pair of natural numbers should be an object $(m, n)$ from which you can determine two natural numbers in a predetermined order. This means that we do not want $(m, n)$ and $(n, m)$ to be the same object. The first ordered pair, $(m, n)$, means we have two natural numbers. First $m$, then $n$. The second ordered pair $(n, m)$ means we have first $n$, then $m$. A set of two natural numbers $\{X, Y\}$ is not an ordered pair because we it simply tells us we have two objects, without a pre determined order; we simply have objects $X, Y$ and they are not ordered.

To solve this, we give a method of coding an ordered pair of natural numbers using odd/even numbers to represent the first/second component respectively. Given any set number $X$, we can associate to it the odd number $s(X) \oplus 1$, and the even number $s(X \oplus 1)$. For example, 0 is associated to the odd number $s(0) \oplus 1=1$ and the even number $s(0 \oplus 1)=2$. The number 1 is associated the odd number $s\{0\} 1=\{1\} \oplus 1=2 \oplus 1=3$ and the even number $s(1 \oplus 1)=s(2)=4$. In general, the number $k$ is associated to the $k+1$-st odd and even numbers $2 k+1$ and $2(k+1)$, as shown in the table below.

$\begin{array}{ccc}X & \text { Odd } & \text { Even } \\ 0 & 1 & 2 \\ 1 & 3 & 4 \\ 2 & 5 & 6 \\ 3 & 7 & 8 \\ 4 & 9 & 10 \\ 5 & 11 & 12 \\ 6 & 13 & 14 \\ \vdots & \vdots & \vdots\end{array}$

Transforming a set number $X$ to its odd representation does alter the form of the set. All we do is displace the objects of $X$ one unit up, to get $s(X)$. Then, we add the object 0 to the set $s(X)$ to obtain $s(X) \oplus 1=s(X) \oplus\{0\}$. When we add the object 0 , it will not alter anything else because $0 \notin s(X)$, so we simply add the object to the set. For example, the set number $5=\{0,2\}$ is sent to the odd number $s(5) \oplus 1=\{0 \oplus 1,2 \oplus 1\} \oplus 1=\{1,3\} \oplus\{0\}=\{0,1,3\}$. Take another example, the set number $13=\{0,2,3\}$ that is sent to the odd representation $s(13) \oplus 1=\{0 \oplus 1,2 \oplus 1,3 \oplus 1\} \oplus\{0\}=\{1,3,4\} \oplus\{0\}=\{0,1,3,4\}$. Given an odd number, $A$, we can easily see what set number it represents because an odd number is simply a set number $A$ with $0 \in A$. To find the set number $X$, such that $A=s(X) \oplus 1$, we simply have to take away the object 0 , from $A$, and displace the objects one unit down. Doing this to the odd number $\{0,1,3,4\}$, of our last example, we get $13=\{1-1,3-1,4-1\}=\{0,2,3\}$.

This situation is only slightly different in the even case because the even number representing the set number $X$ is not a displacement of $X$. However this is not a problem because it is still obtainable. That is, we can still go back an forth between a set number $X$ and its even representation. A set number $X$ is represented by the even number $s(X \oplus 1)$ which is obtained by applying $\oplus 1$ to the set $X$, and then displacing the objects of $X \oplus 1$ one unit up. For example, the set number $7=\{0,1,2\}$ is assigned the even number $16=s(\{0,1,2\} \oplus 1)=s(\{0,1,2\} \oplus\{0\})=s\{3\}=\{3 \oplus 1\}=\{4\}$. An even number is a set number $B$ such that $0 \notin B$, so that all the objects of $B$ can be displaced at least one unit down. So now if we have an even number $B$, we can find the set number $X$ it is representing. We simply have to displace the objects of $B$ one unit down, and this gives us $X \oplus 1$. Then $B$ is representing the set number $X$, which is the predecessor of $X \oplus 1$. For example, the number $10=\{1,3\}$ is the even representation of $4=5-1$ because $\{1-1,3-1\}=\{0,2\}=5$.

The ordered pair $(m, n)$ is a set number of one odd and one even number, $\{2 m+1,2(n+1)\}$. This allows us to differentiate the two components. We will use the convention that the odd number is the first component of the ordered pair, while the 
even number is used for the second component of the ordered pair. If $P=\{A, B\}$ is an ordered pair, we use the convention that the first component is given by the odd number, $A$, and the second component is given by the even number, $B$. An ordered pair $P=(0, n)$, with 0 in the first component, is a set number $P=\{1, B\}$, with $0 \notin B$. If we have 1 in the first component, $P=(1, n)$, then $P=\{3, B\}$. If we have 2 in the first component, $P=(2, n)$, then $P=\{5, B\}$, etc. In general if the $k+1$-th odd number is an element of $P=\{2 k+1, B\}$, it is representing an ordered pair with $k$ in the first component, $P=(k, n)$. We use even numbers to represent the second component. If 0 is in the second component, $P=(m, 0)$, then $2 \in P$ so we have $P=\{A, 2\}$, where $0 \in A$. To represent $P=(m, 1)$, an ordered pair with 1 in the second component, we have $P=\{A, 4\}$. An ordered pair $P$ with 2 in the second component contains the third even number, 6 , etc. In general, if the $k+1$-th even number is an element of $P=\{A, 2(k+1)\}$, it represents an ordered pair $P=(m, k)$.

We define an ordered pair $(m, n)$, of natural numbers $m, n$, to be the set number $2^{2 m+1}+2^{2(n+1)}=\{2 m+1,2(n+1)\}$, where $m, n \in \mathbb{N}$. The set number representing $(0,0)$ is $\{1,2\}=2^{2(0)+1}+2^{2(0+1)}=6$. For another example, the ordered pair $(4,5)$ is represented by the natural number $2^{2(4)+1}+2^{2(5+1)}=2^{9}+2^{12}$. In summary, $P=\{A, B\} \in \mathbb{N}$, with $0 \in A$ and $0 \notin B$, represents the ordered pair $(m, n)$, where $m, n \in \mathbb{N}$ are the unique natural numbers that satisfy $s(m) \oplus 1=A$ and $s(n \oplus 1)=B$. We find $m=\frac{A-1}{2}$ and $n=\frac{B}{2}-1$, where $\frac{X}{2}=s^{-1}(X)$. The function $s^{-1}$ sends the elements of $X$ to their predecessor, $s^{-1}(X)=\{x-1\}_{x \in X}$. There exists a numeric table describing this rule, in general.

Definition 8. We define a family of sets,

$$
\begin{aligned}
(0,)= & \left\{6,18,66,258,1026, \ldots, 2+2^{2(n+1)}, \ldots\right\} \\
(1,)= & \left\{12,24,72,264,1032, \ldots, 8+2^{2(n+1)}, \ldots\right\} \\
(2,)= & \left\{36,48,96,288,1056, \ldots, 32+2^{2(n+1)}, \ldots\right\} \\
\vdots & \vdots \\
(m,)= & \left\{2^{2 m+1}+4,2^{2 m+1}+16, \ldots, 2^{2 m+1}+2^{2(n+1)}, \ldots\right\}
\end{aligned}
$$

Any element $x \in \bigcup_{i}(i$,$) , in the above family of sets, is an ordered pair. The ordered pair (m, n)$ is the $n+1$-st element of the set $(m$,$) . A finite relation is a finite subset R \subset \bigcup_{i}(i$,$) ; elements of R$ are called components.

There are a few important remarks to be made. Every ordered pair of natural numbers is identified with a unique natural number. Two ordered pairs are the same if and only they are represented by the same set number. And, every natural number representing an ordered pair is a multiple of 6 (the converse is obviously not true).

We have an easy way of knowing what number represents $(m, n)$. An ordered pair $(0, n)$ is any element of the set $(0$,$) . The$ ordered pair $(0,0)$ is represented by $6=2^{2(0)+1}+2^{2(0+1)}$, and $(0,1)$ is $18=2^{2(0)+1}+2^{2(1+1)}$. The third number of the set $(0$,$) represents the ordered pair (0,2)$, etc. An element of $(1$,$) is an ordered pair of the form (1, n)$. The ordered pair $(1,0)$ is represented by $12=2^{2(1)+1}+2^{2(0+1)}$, the first object of $(1$,$) . The ordered pair (1,1)$ is $24=2^{2(1)+1}+2^{2(1+1)}$, the second object of $(1$,$) . The third object of (1$,$) represents the ordered pair (1,2)$, etc.

Now, we are able to make an important jump. This is the second part of our definition. A finite collection of ordered pairs is a natural number,

$$
\left\{\left\{A_{1}, B_{1}\right\},\left\{A_{2}, B_{2}\right\}, \ldots,\left\{A_{n}, B_{n}\right\}\right\}=2^{2^{A_{1}}+2^{B_{1}}}+2^{2^{A_{2}}+2^{B_{2}}}+\cdots+2^{2^{A_{n}}+2^{B_{n}}}
$$

where $A_{i}$ are odd and the $B_{i}$ are even. A set of ordered pairs is a relation, as is usual. We are able to store the information of a finite relation in a single natural number, and the structure is again obtainable from that number. The relation 


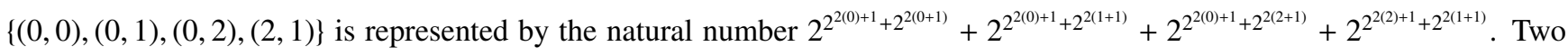
finite relations are the same if and only if they are represented by the same natural number. For another example, take the relation $\{(2,1),(2,2),(4,2),(4,4)\}$ given by the natural number $2^{2^{5}+2^{4}}+2^{2^{5}+2^{6}}+2^{2^{9}+2^{6}}+2^{2^{9}+2^{10}}$. Now that we are able to represent any finite relation, as a natural number, we are able to describe finite functions as natural numbers as well.

\subsection{Concrete Functions}

This method allows us to represent a finite function of natural numbers, as a natural number. Going back to our definition of relation, we simply require that no odd number be repeated. A finite function is represented by a set number of the form (7), where all the $A_{i}$ are distinct.

Definition 9. A function is a natural number of the form $\left\{\left\{A_{1}, B_{1}\right\},\left\{A_{2}, B_{2}\right\}, \ldots,\left\{A_{n}, B_{n}\right\}\right\}=2^{2^{A_{1}}+2^{B_{1}}}+2^{2^{A_{2}}+2^{B_{2}}}+\cdots+2^{2^{A_{n}}+2^{B_{n}}}$, where all the $A_{i}$ are distinct odd numbers and $B_{i}$ are even numbers. A function is called bijective if, additionally, all the $B_{i}$ are distinct. Every element of the function is an arrow component of the function. A function $f$ maps $m \mapsto n$ if and only if $2^{2 m+1}+2^{2(n+1)} \in f$.

A permutation $\{0,1,2, \ldots, n\} \rightarrow\{0,1,2, \ldots, n\}$ is particularly easy to identify. It is a set of $n+1$ ordered pairs, in which every element of $\{1,2,3,4, \ldots, 2 n, 2 n+1,2(n+1)\}$ appears in exactly one ordered pair. Examples of permutations are

$$
\begin{aligned}
\{\{1,2\},\{3,4\}\} & =2^{2^{1}+2^{2}}+2^{2^{3}+2^{4}} \\
\{\{1,3\},\{2,4\}\} & =2^{2^{1}+2^{3}}+2^{2^{2}+2^{4}} \\
\{\{1,2\},\{3,4\},\{5,6\},\{7,8\}\} & =2^{2^{1}+2^{2}}+2^{2^{3}+2^{4}}+2^{2^{5}+2^{6}}+2^{2^{7}+2^{8}} \\
\{\{1,6\},\{3,8\},\{5,2\},\{7,4\}\} & =2^{2^{1}+2^{6}}+2^{2^{3}+2^{8}}+2^{2^{5}+2^{2}}+2^{2^{7}+2^{4}} \\
\{\{1,4\},\{3,10\},\{5,6\},\{7,8\},\{9,2\}\} & =2^{2^{1}+2^{4}}+2^{2^{3}+2^{10}}+2^{2^{5}+2^{6}}+2^{2^{7}+2^{8}}+2^{2^{9}+2^{2}} \\
\{\{1,6\},\{3,8\},\{5,2\},\{7,10\},\{9,4\}\} & =2^{2^{1}+2^{6}}+2^{2^{3}+2^{8}}+2^{2^{5}+2^{2}}+2^{2^{7}+2^{10}}+2^{2^{9}+2^{4}}
\end{aligned}
$$

The first permutation is the identity permutation (0)(1). The second set number is representing the one-cycle permutation $(0,1)$. The third and fourth numbers represent $(1)(2)(3)(4)$ and $(0,2)(1,3)$, respectively. The fifth and sixth permutations are $(0,1,4),(2)(3)$, and $(0,2)(1,3,4)$. We provide a linear order to the set of finite functions, and in particular permutations.

This order is well behaved in several ways. If $f:\{0,1,2, \ldots, m\} \rightarrow\{0,1,2, \ldots, m\}$, and $g:\{0,1,2, \ldots, n\} \rightarrow\{0,1,2, \ldots, n\}$ are permutations and $m<n$, then the representation of $f$ is smaller than the representation of $g$. For now, we conclude this section with the following remark. This representation is not very good if we want a representation that measures how much movement a permutation causes. This manner of assigning natural numbers to functions makes a distinction between functions with the same structure. Concrete functions with the same structure are associated to different natural numbers if we use different natural numbers as objects. For example, the functions $f, g$ defined by $f(0)=0$ and $g(1)=1$ have the same structure but are assigned different numbers. In the following section we address this issue and we are able to resolve. We will assign a natural number to each finite function, in such a way that two functions are represented by the same number if and only if they have the same structure (this will be our definition of equivalent structure between two functions). A method is given for assigning natural numbers to abstract functions. Concrete functions will also be assigned a number representation that does not depend on the objects of the function, and only depends on the structure of the function. To do this, we find a way to forget the numerical values of the objects of the concrete function, turning it into an abstract function. Then we find the number representation of that abstract function. 


\subsection{Abstract Functions}

Consider the permutations $(1,2)(3,4)$ and $(1,3)(2,4)$. These will be represented by the numbers $2^{2^{3}+2^{6}}+2^{2^{5}+2^{4}}+2^{2^{7}+2^{10}}+2^{2^{9}+2^{8}}$ and $2^{2^{3}+2^{8}}+2^{2^{7}+2^{4}}+2^{2^{5}+2^{10}}+2^{2^{9}+2^{6}}$, respectively. These numbers are different and we would like to change this. We would like to make a good definition, modulo the structure, so that the two functions above are assigned the same natural number. It would be advantageous to number finite functions in such a manner that functions with the same structure will be represented by the same natural number. Let $f: A \rightarrow B$ a concrete function, where $A, B \in \mathbb{N}$. Our first step in solving our problem is to forget the numeric value assigned to the elements of the components. This means that the sets $A, B$ are no longer thought of as having concrete elements (natural numbers). We will think of the elements of $A$ and $B$ as abstract objects with a function defined on them. We need to know how many distinct elements are in $A \cup B$. For example, the function $f$ defined by

$$
\begin{aligned}
f(2) & =2 \\
f(5) & =6 \\
f(6) & =5 \\
f(8) & =6 \\
f(10) & =15
\end{aligned}
$$

depends on the distinct objects $2,5,6,8,10,15$ and it will be considered an abstract function $f^{*}$ defined by

$$
\begin{aligned}
f^{*}(a) & =a \\
f^{*}(b) & =c \\
f^{*}(c) & =b \\
f^{*}(d) & =c \\
f^{*}(p) & =q
\end{aligned}
$$

Now, we must find a way of assigning a natural number $N_{f^{*}}$ to the abstract function $f^{*}$, in a sufficiently reasonable manner. To do this, we must go back to the realm of numeric values. Let us take a fixed bijection $\eta:\{a, b, c, d, p, q\} \rightarrow\{0,1,2,3,4,5\}$, and call it a naming function of $f^{*}$. Using the procedure of the last section, we have a representation $N_{f^{*}}(\eta) \in \mathbb{N}$ that depends on the naming function $\eta$ and the abstract function $f^{*}$. Now consider the set of all representations $\left\{N_{f}(\eta)\right\}_{\eta}$; let $\eta$ variable over all possible naming functions. In our example we have 6 ! possibilities.

To find the modulo-structure representation of a concrete function $f$, we first find the abstract function $f^{*}$ corresponding to $f$, then we proceeded to find all the possible naming functions of $f^{*}$. There is a total of \# $(A \cup B)$ ! naming functions. Each naming function $\eta$ provides a representation $N_{f^{*}}(\eta)$, so that we have a set of representations $\left\{N_{f^{*}}(\eta)\right\}_{\eta}$.

Definition 10. Let $f$ be a concrete function and $f^{*}$ its corresponding abstract function. Their exists at least one naming, $\rho$, such that $N_{f^{*}}(\rho)$ is equal to the maximum element of the set $\left\{N_{f^{*}}(\eta)\right\}_{\eta}$. This maximum is the modulo-structure representation of $f$, and we use the symbol $N_{f^{*}}=N_{f^{*}}(\rho)$.

Let $f^{*}$ and $g^{*}$ two abstract functions such that $N_{f^{*}}(\eta)=N_{g^{*}}(\mu)$, for some naming functions $\eta$ of $f^{*}$ and $\mu$ of $g^{*}$. Then $f^{*}=g^{*}$ and $\eta, \mu$ are two naming functions of the same abstract function and we say they are equivalent naming functions of $f^{*}$. We can state this differently. The sets of representations for $f^{*}, g^{*}$ are disjoint if $f^{*}, g^{*}$ are different functions; $f^{*} \neq g^{*}$ implies 
$\left\{N_{f^{*}}(\eta)\right\}_{\eta} \cap\left\{N_{g^{*}}(\mu)\right\}_{\mu}=\emptyset$. This gives us a good representation of abstract functions as natural numbers. We have a numeric representation of finite functions, because $\left\{N_{f^{*}}(\eta)\right\}_{\eta}$ is a natural number and two functions are assigned different numbers if they have different structure. Therefore, we have an ordering of finite functions in the sense that two finite functions can be compared, $f<g$, in terms of a linear order.

The representation of a function is a large natural number because $\#\left\{N_{f^{*}}(\eta)\right\}_{\eta}=\left(\#\left(\operatorname{Dom}\left(f^{*}\right) \cup \operatorname{Im}\left(f^{*}\right)\right)\right)$ !. If $f^{*}$ is a permutation of $k$ objects, the representation of $f^{*}$ is a natural number that is the sum of $k$ ! distinct powers of 2 . The representation of a permutation of 10 objects would be a natural number somewhere close to $10^{10^{230}}$. We can make this representation smaller, and the order of the functions will be invariant. The fact that the sets of representations are disjoint, is a great advantage we use in Definition 10. We chose to represent the function with the maximum of the set, for the following reason. Let $A \cap B=\emptyset$, then the order relation of the maximum elements, $\max (A)<\max (B)$, determines the order relation $A<B$. Since the sets of representations are disjoint for different functions, we conclude that representing $f$ with the set of representations, $\left\{N_{f^{*}}(\eta)\right\}_{\eta}$, or with the maximum element, $N_{f^{*}}=\max \left\{N_{f^{*}}(\eta)\right\}_{\eta}$, gives us the same order.

Now we define equivalent objects of a finite function $f: A \rightarrow B$ of $n$ objects; $n=\#(A \cup B)$. Suppose we have two canonical naming functions $\rho_{1}, \rho_{2}: \rightarrow\{0,1,2, \ldots, n-1\}$ so that $N_{f^{*}}=N_{f^{*}}\left(\rho_{1}\right)=N_{f^{*}}\left(\rho_{2}\right)$. We are supposing the naming functions are not equal, so that we have $\rho_{1}(x) \neq \rho_{2}(x)$, for some $x \in A \cup B$. Also, naming functions are bijections, so we know there exists $y \neq x$ such that $\rho_{1}(y)=\rho_{2}(x)$. We will say $x, y$ are equivalent objects because there are two distinct canonical naming functions $\rho_{1}, \rho_{2}$ that assign the same numerical value to $x, y$.

Definition 11. Let $f: A \rightarrow B$ a finite function. Two objects $x, y \in A \cup B$ are equivalent if there exist canonical naming functions $\rho_{1}, \rho_{2}$ such that $k=\rho_{1}(x)=\rho_{2}(y)$, for some $k \in A \cup B=\{0,1,2, \ldots, n-1\}$. This is an equivalence relation on the set of objects $A \cup B$.

This method has given us two things. We are able to number the set of all finite functions (modulo structure), and we are also provided with a canonical naming function on the objects $\operatorname{Dom}(f) \cup \operatorname{Im}(f)$. We can order the set of abstract finite permutations, and we can also order the elements of any abstract finite permutation. Most importantly these orders are well behaved in several ways. In this work, we focus on the ordering of finite permutations, and a general exposition of finite functions is left for future work. Nonetheless, we do some examples of general functions. Let us find the representation of the first finite functions, to get an intuitive grasp of how functions are ordered.

Our first example is of course the trivial function $f_{0}$ that sends $a \rightarrow a$. This function depends of a single object so we use the set $\{0\}$ to name the set of objects $\{a\}$. We have to recall the definition of ordered pair, and specifically we said the ordered pair $0 \rightarrow 0$ is represented by the number $6=2^{1}+2^{2}$. We use the odd number to represent the preimage and the even number to represent the image; a 0 in the preimage means 1 is an element of the ordered pair and a 0 in the image means 2 is an element of the ordered pair. Our function consists of one component. Its only element is 6 , so $N_{f_{0}}=2^{2^{1}+2^{2}}$. Let us continue to represent functions. We will try to construct all finite functions in order of their representation, so the next logical choice is the function $f_{1}$ defined by one component, $f_{1}(a)=b$. In this case we have two objects so a naming of this function is a bijection $\{a, b\} \rightarrow\{0,1\}$. If we choose the naming $a=0$ and $b=1$ we get the representation $2^{2^{1}+2^{4}}$. If we give instead the naming $a=1$ and $b=0$ then the representation is $2^{2^{3}+2^{2}}$. We conclude that the canonical representation of $f_{1}$ is the number $N_{f_{1}}=2^{2^{1}+2^{4}}$ corresponding to the first naming function $a=0$ and $b=1$. These are the only two possible abstract functions of one component; namely $f_{0}(0)=0$ (trivial function) and $f_{1}(0)=1$ (one object sent to a different object).

Now let us consider functions of two components. Bur first let us recall what it is we are trying to do. We know finite functions are ordered isomorphic to $\mathbb{N}$; every finite function is assigned a unique natural number. We can state this in the following simple manner. There is a set of natural numbers $\left\{N_{f}\right\}_{f: \text { finite function }}=\left\{N_{0}, N_{1}, N_{2}, \ldots\right\}$ and we say an element of this set is a finite function. Let us find the first few functions $f_{0}, f_{1}, \ldots$ represented by the first numbers $N_{0}, N_{1}, \ldots$ So far we know the first two functions are the one component functions $N_{0}=2^{2^{1}+2^{2}}$ and $N_{1}=2^{2^{1}+2^{4}}$ from above. If we want to find the next 
function, $f_{2}=N_{2}$, we will have to add a component but we do not want to use more than two objects because that would give us a function represented by a larger number. Consider finite functions of two components, and two objects. There is a total of 3 functions that satisfy this conditions and they are the functions $N_{2}, N_{3}, N_{4}$. The function $N_{2}$ is given by two components that switch the objects in the domain, $f_{2}(a)=b$ and $f_{2}(b)=a$. This is the first time we encounter two equivalent objects for a function. If we give the naming $a=0, b=1$ or the naming $a=1, b=0$ we get the same representation $N_{2}=2^{2^{1}+2^{4}}+2^{2^{2}+2^{3}}$. The next function in order is the identity function on two objects, $f_{3}(a)=a$ and $f_{3}(b)=b$. Again, both objects are equivalent and they give the representation $N_{3}=2^{2^{1}+2^{2}}+2^{2^{3}+2^{4}}$. The function $N_{4}$ is the trivial function that sends two objects to one of the two; the components are $f_{4}(a)=a$ and $f_{4}(b)=a$. The canonical representation $N_{4}=2^{2^{1}+2^{4}}+2^{2^{3}+2^{4}}$ is given by the naming $a=1$ and $b=0$. The first summand represents $f_{4}(0)=1$ and the second summand represents $f_{4}(1)=1$. We can easily verify the alternative naming function would give us a smaller representation. If we give the naming $a=0$ and $b=1$, the corresponding representation of $f_{4}$ is $2^{2^{1}+2^{2}}+2^{2^{3}+2^{2}}$. Notice that the function that seems to cause more movement, $f_{2}$, is represented by the smallest number of the three. The function that sends everything to $a$ is the largest of the three, and the identity is the middle number. This observation will be important in the special case of ordering permutations. Now we can consider functions of two components and three objects, the next functions in our order $N_{5}, N_{6}, N_{7}$. We give each function with its canonical naming, and then some of the other non canonical representations.

$f_{5}(a)=a, f_{5}(b)=c$ has canonical naming $a=2, b=0, c=1$ giving the ordered pairs $(2,2),(0,1)$ with representation

$$
N_{5}=2^{2^{1}+2^{4}}+2^{2^{5}+2^{6}}
$$

Other, non canonical, naming functions are $a=2, b=1, c=0$ with representation $2^{2^{3}+2^{2}}+2^{2^{5}+2^{6}} ; a=0, b=1, c=2$ with representation $2^{2^{1}+2^{2}}+2^{2^{3}+2^{6}} ; a=0, b=2, c=1$ with representation $2^{2^{1}+2^{2}}+2^{2^{5}+2^{4}} ; a=1, b=2, c=0$ with representation $2^{2^{3}+2^{4}}+2^{2^{5}+2^{2}}$, etc.

$$
f_{6}(a)=c, f_{6}(b)=c \text { has canonical naming } a=0, b=1, c=2 \text { giving the ordered pairs }(0,2),(1,2) \text { with representation }
$$

$$
N_{6}=2^{2^{1}+2^{6}}+2^{2^{3}+2^{6}}
$$

The naming $a=1, b=0, c=2$ is also canonical; $a, b$ are equivalent objects of $f$. Other, non canonical, representations are $a=2, b=1, c=0$ with representation $2^{2^{5}+2^{2}}+2^{2^{3}+2^{2}} ; a=2, b=0, c=1$ with representation $2^{2^{5}+2^{4}}+2^{2^{1}+2^{4}}$. etc.

$$
f(a)=b, f(b)=c \text { has canonical naming } a=1, b=2, c=0 \text { giving ordered pairs }(1,2),(2,0) \text { with representation }
$$

$$
N_{7}=2^{2^{3}+2^{6}}+2^{2^{5}+2^{2}}
$$

Other, non canonical, representations are $a=0, b=1, c=2$ with representation $2^{2^{1}+2^{4}}+2^{2^{3}+2^{6}} ; a=0, b=2, c=1$ with representation $2^{2^{1}+2^{6}}+2^{2^{5}+2^{4}} ; a=1, b=0, c=2$ with representation $2^{2^{3}+2^{2}}+2^{2^{1}+2^{6}} ; a=2, b=1, c=0$ with representation $2^{2^{5}+2^{4}}+2^{2^{3}+2^{2}} ; a=2, b=0, c=1$ with representation $2^{2^{5}+2^{2}}+2^{2^{1}+2^{4}}$, etc.

We have so far found the first eight numbers $N_{0}, N_{1}, \ldots, N_{7}$, and now we wish to find the next numbers representing functions. Here, we have to be careful. There is one function of two components and four objects. However, it is not next in order, because the functions of three components and three objects have smaller representation. We see that the order of functions

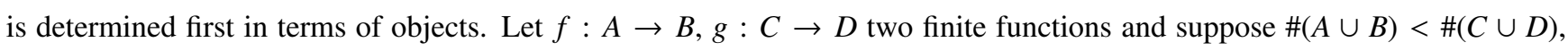
then $f<g$. If $\#(A \cup B)=\#(C \cup D)$ we check the number of components; the function with more components has larger representation so $\#(f)<\#(g)$ implies $f<g$. Let $A_{n}^{m}$ a finite function of $n$ objects and $m$ components. Then the following inequalities are true 


$$
A_{1}^{1}<A_{2}^{1}<A_{2}^{2}<A_{3}^{2}<A_{3}^{3}<A_{4}^{2}<A_{4}^{3}<A_{4}^{4}<A_{5}^{3}<A_{5}^{4}<A_{5}^{5}<A_{6}^{3}<A_{6}^{4}<A_{6}^{5}<A_{6}^{6}<\cdots
$$

The following table which states the number of functions with $n$ objects and $m$ components. There is one function of one object and one component $(a \rightarrow a)$. There is one function of two objects and one component $(a \rightarrow b)$. There are three functions of two objects and two components. We found three functions of three objects and two components, etc.

$\begin{array}{ccc}\# F & \# O & \# C \\ 1 & 1 & 1 \\ 1 & 2 & 1 \\ 3 & 2 & 2 \\ 3 & 3 & 2 \\ 7 & 3 & 3 \\ 1 & 4 & 2 \\ 9 & 4 & 3 \\ & 4 & 4 \\ 3 & 5 & 3 \\ & 5 & 4 \\ & 5 & 5 \\ 1 & 6 & 3 \\ & 6 & 4 \\ & 6 & 5 \\ & 6 & 6\end{array}$

If $f, g$ have the same number of objects, and the same number of components, then we have to find their canonical representation and the order relation $N_{f}<N_{g}$ determines the order relation $f<g$. Therefore, to compare two finite functions, it is sufficient to compute their canonical representations and compare these numbers. However, knowing which $N_{k}$ represents a finite function is more complicated. We can easily find the canonical representation $N_{f}$ but if we want to know its position in the order, we need more information than just its canonical representation. We have to know how many functions there are of less objects, and how many functions of the same number of objects but of less components. Then, we need to find the canonical representation of all functions with the same number of components and objects. In the table above, we state there are seven functions of three components and three objects. We now provide these seven functions, and for simplicity of lecture and exposition we give arrows defining these functions. For example, the function defined by the three components $f(a)=f(b)=f(c)=a$ is the set of arrows of the last column.

$\begin{array}{ccccccc}N_{8} & N_{9} & N_{10} & N_{11} & N_{12} & N_{13} & N_{14} \\ a \rightarrow b & a \rightarrow b & a \rightarrow a & a \rightarrow a & a \rightarrow a & a \rightarrow a & a \rightarrow a \\ b \rightarrow c & b \rightarrow a & b \rightarrow c & b \rightarrow b & b \rightarrow a & b \rightarrow a & b \rightarrow a \\ c \rightarrow a & c \rightarrow a & c \rightarrow b & c \rightarrow c & c \rightarrow c & c \rightarrow b & c \rightarrow a\end{array}$

Any function of three components and three objects is equivalent to one of these seven. These functions are next in the canonical ordering of finite functions; they are represented by the numbers $N_{8}, N_{9}, \ldots, N_{14}$. To know which of these seven functions is $N_{8}$, we have to find the canonical representation of all seven and the one with smallest canonical representation 
is the function $N_{8}$, then the function $N_{9}$ is the function with second smallest representation, etc. Of these seven functions, the one with largest representation is the function $N_{14}$. Here we give them in order from smallest to largest (left to right). We leave as an exercise for the reader to verify the canonical representations of these functions.

$$
\begin{aligned}
& N_{8}=2^{2^{1}+2^{4}}+2^{2^{3}+2^{6}}+2^{2^{5}+2^{2}} \\
& N_{9}=2^{2^{1}+2^{6}}+2^{2^{3}+2^{6}}+2^{2^{5}+2^{4}} \\
& N_{10}=2^{2^{1}+2^{4}}+2^{2^{3}+2^{2}}+2^{2^{5}+2^{6}} \\
& N_{11}=2^{2^{1}+2^{2}}+2^{2^{3}+2^{4}}+2^{2^{5}+2^{6}} \\
& N_{12}=2^{2^{1}+2^{2}}+2^{2^{3}+2^{6}}+2^{2^{5}+2^{6}} \\
& N_{13}=2^{2^{1}+2^{4}}+2^{2^{3}+2^{6}}+2^{2^{5}+2^{6}} \\
& N_{14}=2^{2^{1}+2^{6}}+2^{2^{3}+2^{6}}+2^{2^{5}+2^{6}}
\end{aligned}
$$

To find the canonical representation of $N_{8}$, we observe the objects are all equivalent. Let $a=2$, then we obviously have to make $b=0$ and $c=1$, because we have to maximize the term where $a$ is image. The naming functions $b=2, a=1, c=0$ and $c=2, b=1, a=0$ also give the canonical representation. The canonical naming function of $N_{9}$ is easy to find, as well. We start by naming $a=2$, since $a$ is the most frequent object. Then we make $b=1$ because $b$ is the object that has more relations with $a$. In $N_{10}$ we first make $a=2$ because $a$ is a fixed point; this ensures we have the term $2^{5}+2^{6}$. The objects $b, c$ are equivalent in the function $N_{10}$ because we have the two canonical naming functions $a=2, b=1, c=0$ and $a=2, b=0, c=1$. The rest of the canonical naming functions are easily found.

Now consider the function of two components and four objects defined by $f_{15}(a)=c$ and $f_{15}(b)=d$. The objects in the image have priority for being assigned larger numbers, so we start with naming $c=3$ because $c$ is in the image. Now, things change between choosing $a, b, d$ for the value 2 . Instead of assigning 2 to $d$, which is also in the image, we want to use the object that is related to $c=3$. That would be the object $a=2$. Then, we trivially assign the values $d=1$ and $b=0$. The components of the function are the ordered pairs $(2,3)$ and $(0,1)$ stating $f_{15}(2)=3$ and $f_{15}(0)=1$. The set of these ordered pairs is the canonical representation $N_{15}=2^{2^{1}+2^{4}}+2^{2^{5}+2^{8}}$; the summand $2^{2^{1}+2^{4}}$ represents the pair $(0,1)$ and the second summand $2^{2^{5}+2^{8}}$ represents the pair (2,3). The naming function $d=3, b=2, c=1, a=0$ gives components $(0,1)$ and $(2,3)$ so that this is also a canonical naming function. Equivalent objects are those that can be assigned the same numerical value under different canonical naming functions. Therefore, $a, b$ are equivalent and $c, d$ are equivalent.

Next in order we have the functions of three components and four objects. Each of these nine functions is represented by one of the numbers $N_{16}, N_{17}, \ldots, N_{24}$. Any function of three components and four objects is equivalent to one of these nine.

$\begin{array}{ccccccccc}N_{16} & N_{17} & N_{18} & N_{19} & N_{20} & N_{21} & N_{22} & N_{23} & N_{24} \\ a \rightarrow c & a \rightarrow b & a \rightarrow b & a \rightarrow c & a \rightarrow d & a \rightarrow a & a \rightarrow a & a \rightarrow a & a \rightarrow a \\ b \rightarrow a & b \rightarrow a & b \rightarrow d & b \rightarrow c & b \rightarrow d & b \rightarrow c & b \rightarrow d & b \rightarrow b & b \rightarrow a \\ c \rightarrow d & c \rightarrow d & c \rightarrow d & c \rightarrow d & c \rightarrow d & c \rightarrow d & c \rightarrow d & c \rightarrow d & c \rightarrow d\end{array}$

The smallest of these nine functions is $N_{16}=2^{2^{1}+2^{6}}+2^{2^{5}+2^{8}}+2^{2^{7}+2^{4}}$ given by the canonical naming function $a=2, b=0$, $c=3, d=1$. The next function is $N_{17}=2^{2^{1}+2^{4}}+2^{2^{5}+2^{8}}+2^{2^{7}+2^{6}}$ with canonical naming function $a=3, b=2, c=0, d=1$ and $a, b$ are equivalent objects. The third is $N_{18}=2^{2^{1}+2^{6}}+2^{2^{3}+2^{8}}+2^{2^{5}+2^{8}}$ under the naming $a=0, b=2, c=1, d=3$. Next is the function $N_{19}=2^{2^{3}+2^{8}}+2^{2^{5}+2^{8}}+2^{2^{7}+2^{2}}$ with the naming function $a=2, b=1, c=3, d=0$ and $a, b$ are equivalent objects. The function $N_{20}=2^{2^{1}+2^{8}}+2^{2^{3}+2^{8}}+2^{2^{5}+2^{8}}$ has naming $a=2, b=1, c=0, d=3$ and $a, b, c$ are equivalent objects. The function $N_{21}=2^{2^{3}+2^{6}}+2^{2^{5}+2^{2}}+2^{2^{7}+2^{8}}$ is given by $a=3, b=1, c=2, d=0$. We have $N_{22}=2^{2^{1}+2^{6}}+2^{2^{3}+2^{6}}+2^{2^{7}+2^{8}}$ with 
$a=3, b=1, c=0, d=2$ and $b, c$ equivalent. The second largest is $N_{23}=2^{2^{1}+2^{4}}+2^{2^{5}+2^{6}}+2^{2^{7}+2^{8}}$ with naming $a=3, b=2$, $c=0, d=1$ and $a, b$ equivalent. Finally, we have $N_{24}=2^{2^{1}+2^{4}}+2^{2^{5}+2^{8}}+2^{2^{7}+2^{8}}$ with $a=3, b=2, c=0, d=1$. By now we can see that is not trivial to find the canonical naming function of a finite function, in the general case. In this section we have to make careful observations to calculate the canonical naming functions, without having to find all possible representations. We will have two main problems to solve in the general case, and we will treat these computational strategies in future work. These are 1) finding the canonical naming function of any finite function, and 2) we need to know how many distinct abstract functions of $n$ objects and $m$ components. In the next section we will study the suborder of finite permutations, and it will prove much easier to work with.

At this point the next functions in the order of all finite functions, are functions of four objects and four components. We leave the general analysis for future work. Instead, let us find the next after those; functions of three components and five objects. There is a total of three such functions.

$$
\begin{array}{lll}
a \rightarrow a & a \rightarrow b & a \rightarrow d \\
b \rightarrow d & b \rightarrow d & b \rightarrow d \\
c \rightarrow p & c \rightarrow p & c \rightarrow p
\end{array}
$$

There is one function of three components and six objects.

$$
\begin{aligned}
& a \rightarrow d \\
& b \rightarrow p \\
& c \rightarrow q
\end{aligned}
$$

We wish to find the representation of our example $f^{*}$ on objects $a, b, c, d, p, q$. We have five components, so our representation will be a set of five natural numbers. In the general case it is not easy to construct the largest possible representation, without having to construct several possible representations. We have to find a canonical naming function. We know such a naming function will have to assign $\eta(a)=5$. This guarantees we have $2^{11}+2^{12} \in N_{f^{*}}(\eta)$ representing $f^{*}(a)=a$. There is no object that has a relation with $a$, so we must find out which object, of the remaining objects $b, c, d, p, q$, will be assigned the value 4 . If we make $\eta(c)=4$ we maximize the representation because we will have two components with the power $2^{10}$; namely the components $f^{*}(b)=c$ and $f^{*}(d)=c$. We choose $\eta(b)=3$ instead of $\eta(d)=3$ because $b$ is related to $c$ by two components. This leaves us with $\eta(d)=2$. Now we have to assign $q=1$ and $p=0$. The canonical representation of

$$
\begin{aligned}
f^{*}(a) & =a \\
f^{*}(b) & =c \\
f^{*}(c) & =b \\
f^{*}(d) & =c \\
f^{*}(p) & =q
\end{aligned}
$$

under the canonical naming $\eta$ is

$$
N_{f^{*}}=2^{2^{1}+2^{4}} 2^{2^{5}+2^{10}}+2^{2^{7}+2^{10}}+2^{2^{9}+2^{8}}+2^{2^{11}+2^{12}}
$$

and there are no equivalent objects. 


\subsection{Finite Permutations}

The suborder of permutations is much easier to find. First of all, it is well behaved with respect to cardinality. Let $f$ a permutation on $m$ objects and $g$ a permutation on $n>m$ objects, then $N_{f}<N_{g}$. Furthermore, permutations are ordered by complexity. We say $f$ has size $m$ and $g$ has size $n$. Given permutations $f, g$ of the same size, then we can order these permutations and the interpretation is that a larger number is a simpler permutation. For example, the identity permutation of size $n$ has larger representation than all other permutations of size $n$. We know that the number of distinct abstract permutations of size $n$, is equal to the number of partitions of $n$. Let us order the first few permutations. The permutation of size 1 , is equal to the function $f_{0}$ of one component; we know it is represented by $N_{0}=2^{2^{1}+2^{2}}=2^{6}$. There are two permutations of size 2; these are the functions $N_{2}$ and $N_{3}$. Next we have a total of three permutations of size 3 . These are the functions $N_{8}, N_{10}, N_{11}$. We notice that the smallest of these three numbers, $N_{8}$, represents the permutation that moves every object. The middle permutation is $N_{10}$ and it leaves two objects fixed. The largest, $N_{11}$, represents the identity permutation (leaves all objects fixed). Call these first six permutations $P_{0}=N_{0}, P_{1}=N_{2}, P_{2}=N_{3}, P_{3}=N_{4}, P_{4}=N_{5}, P_{5}=N_{6}$. Let us order the permutations of size 4 . We have five permutations of size 4 . In order, these are

$\begin{array}{ccccc}P_{6} & P_{7} & P_{8} & P_{9} & P_{10} \\ a \rightarrow b & a \rightarrow b & a \rightarrow a & a \rightarrow a & a \rightarrow a \\ b \rightarrow c & b \rightarrow a & b \rightarrow c & b \rightarrow b & b \rightarrow b \\ c \rightarrow d & c \rightarrow d & c \rightarrow d & c \rightarrow d & c \rightarrow c \\ d \rightarrow a & d \rightarrow c & d \rightarrow b & d \rightarrow c & d \rightarrow d\end{array}$

Notice that if two objects are in the same cycle, then they are equivalent. In the first permutation we have the canonical naming function $a=3, b=1, c=0, d=2$. To find this naming function, observe all the objects are equivalent, so we choose $a=3$, without loss of generality. Next, we have to maximize the term where $a$ is in the image. Thus, we define $d=2$. Then to maximize the term where $a$ is in the preimage, we make $b=1$. This leaves us with $c=0$. In the second permutation $a, b$ and $c, d$ are pairs of equivalent objects and a canonical naming function is $a=3, b=2, c=1, d=0$. The third permutation has the naming $a=3, b=2, c=0, d=1$ and $b, c, d$ are equivalent. Next we have the naming $a=3, b=2, c=1 d=0$ with $c, d$ equivalent. The largest of these permutations, $P_{10}$ can have any naming; all objects are equivalent.

$$
\begin{aligned}
& P_{6}=2^{2^{1}+2^{6}}+2^{2^{3}+2^{2}}+2^{2^{5}+2^{8}}+2^{2^{7}+2^{4}} \\
& P_{7}=2^{2^{1}+2^{4}}+2^{2^{3}+2^{2}}+2^{2^{5}+2^{8}}+2^{2^{7}+2^{6}} \\
& P_{8}=2^{2^{1}+2^{4}}+2^{2^{3}+2^{6}}+2^{2^{5}+2^{2}}+2^{2^{7}+2^{8}} \\
& P_{9}=2^{2^{1}+2^{4}}+2^{2^{3}+2^{2}}+2^{2^{5}+2^{6}}+2^{2^{7}+2^{8}} \\
& P_{10}=2^{2^{1}+2^{2}}+2^{2^{3}+2^{4}}+2^{2^{5}+2^{6}}+2^{2^{7}+2^{8}}
\end{aligned}
$$

To find the maximum $N_{f}(\eta)$, over all possible naming $\eta$, we must find the canonical naming providing the largest representation. We wish to maximize the set number $\{\{a, f(a)\},\{b, f(b)\},\{c, f(c)\},\{d, f(d)\}\}$. We know $2^{2(3)+1}+2^{2(3+1)} \in f$ if and only if $f(x)=x$ for some $x \in\{a, b, c, d\}$. If there are no fixed points we look for cycles of two objects, and continue looking for the smallest possible cycle, to assign the largest values of the naming. The largest possible set number that can represent an abstract permutation of four elements is $2^{2^{1}+2^{2}}+2^{2^{3}+2^{4}}+2^{2^{5}+2^{6}}+2^{2^{7}+2^{8}}$, representing the identity permutation (0)(1)(2)(3). The number $N_{f}$ measures and compares the "movement" a permutation causes. If $f$ has more movements than $g$, then $N_{f}>N_{g}$. The more complicated a permutation becomes the smaller its representation becomes (holding fixed the size). Intuitively, assigning larger values to objects in smaller cycles help maximize the representation. 
We will give one more example of permutations before applying the same method to define groups. Let $\sigma$ be the permutation $(a)(b, c)(p, q, r)$ on $\{a, b, c, d, p, q, r\}$. We wish to find a canonical ordering of its elements, and the canonical representation $N_{\sigma}$. It should result in $a=5, b=4, c=3, p=2, q=0, r=1$, or one of its equivalent numbering functions, and

$$
N_{\sigma}=2^{2^{1}+2^{4}}+2^{2^{3}+2^{6}}+2^{2^{5}+2^{2}}+2^{2^{7}+2^{10}}+2^{2^{9}+2^{8}}+2^{2^{11}+2^{12}} \text {. }
$$

In all the equivalent numbering functions, we have $a=0$. We can change $b=3$ and $c=4$. We can also change the values of the objects in the 3-cycle. If we make $q=2$ then we have $p=1$ and $r=0$. If $r=2$, then $q=1$ and $p=0$.

\section{Finite Groups}

Applying the same methods to our definition of group, allows us to represent finite groups as natural numbers. We know, from the first section, that a finite group $G(*)$, defined on an abstract set $G$, is a bijection that assigns permutations of the set $G$ to objects of $G$. We use the notation $\operatorname{Aut}(G)$ to represent the set of permutations on the set $G$. The operation functions are the elements in the image of $*: G \rightarrow \operatorname{Aut}(G)$. Consider a naming $\eta$ of the set $G$. Then the objects of $G$, and the operation functions of $G$ are set numbers. Thus, we can say $*$ is a function of the form $M \rightarrow N$, where $\max (M)<\min (N)$. If the $\operatorname{group}$ has $k$ elements, the domain $M=\operatorname{Dom}(*)$ is the set number $\{0,1,2, \ldots, k-1\}=2^{k}-1$. The image $N=\operatorname{Im}(*)$ is the set number $\{* 0, * 1, * 2, \ldots, * k\}$, where $* x$ are concrete permutations of $\{0,1,2, \ldots, k-1\}$. Notice that the operation functions $N_{* x}$ do not have canonical form because they are concrete functions. We turned the operation function $*(x)$ into a natural number $N_{* x}(\eta)$, by providing the naming function $\eta$. The definition of group we provide satisfies the definition of function, given above. Every finite group is a set number whose elements are ordered pairs. The ordered pairs are sets of two objects; one odd and one even. The first components are odd numbers $2 i+1$, for every $i \in\{0,1,2, \ldots, k-1\}$. The second components are even numbers representing permutations, $2\left(N_{* x}+1\right)$.

We have the same scenario as before, since every naming function $\eta$ defines a natural number $N_{G}(\eta)$, that depends on the group and the naming function of that group. There is a finite number of these representations. The maximum representation is the canonical representation $N_{G}=\max \left\{N_{G}(\eta)\right\}_{\eta}$ of the group $G$. This canonical representation gives us a canonical ordering of the elements of $G$, as well. It behaves much like the representations of permutations. Here we assign the largest value to the identity element, $e=k-1$.

A group is of the form

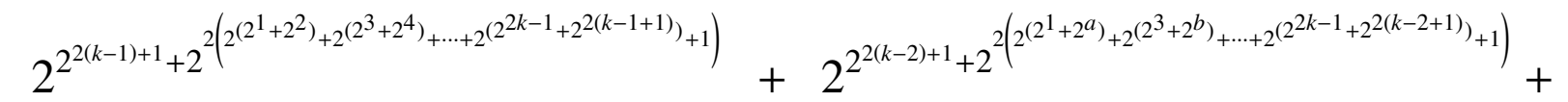

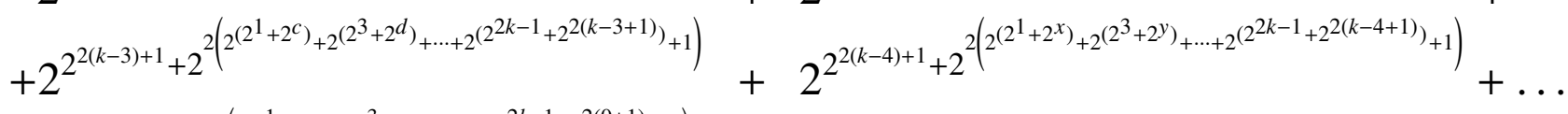

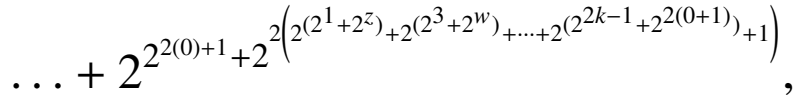

where the $k-1$ numbers $a, b, \ldots$ are distinct elements of $\{2,6,8, \ldots, 2 k-6,2 k-4,2 k\}$, the $k-1$ numbers $c, d, \ldots$ are distinct elements of $\{2,4,8, \ldots, 2 k-6,2 k-2,2 k\}$, the $k-1$ numbers $x, y, \ldots$ are distinct elements of $\{2,4,8, \ldots, 2 k-8,2 k-4,2 k-2,2 k\}$. The numbers $z, w, \ldots$ are distinct elements of $\{4,6, \ldots, 2 k\}$. Also, the $a, c, x, z, \ldots$ are different; all the $b, d, y, w, \ldots$ are different, etc. The first term tells us that the number $k-1$ is assigned to the identity of $G$. 
Not all natural numbers of this form are groups. We additionally require the associative property. Later in this section we will see that verifying the associative property is a straightforward process; we are able to verify this through numeric computation. For that end, we will study the composition of functions, numerically. But before that, we give our main result. With abstract permutations we had a canonical representation, given by a canonical naming of the objects. This naming had equivalent naming functions, if we had equivalent objects. Here we have the same situation, now in the context of groups.

Theorem 7. Given a finite group $G$ of order $k$, We have a naming function $\rho: G \rightarrow\{0,1,2, \ldots, k-1\}$ and a canonical representation $N_{G}=\max _{\eta} N_{G}(\eta)=N_{G}(\rho)$. We say that $\rho$ is the canonical ordering of $G$, and $\rho(e)=k-1$. Two distinct group objects $x, y$ are equivalent if their exists two distinct canonical orderings $\rho_{1}, \rho_{2}$ such that $\rho_{1}(x)=\rho_{2}(y)$.

This gives a well defined linear order on the set of finite groups. Two groups have the same canonical representation if and only if they are isomorphic. This linear order is well behaved with respect to cardinality; $|G|<|H|$ implies $N_{G}<N_{H}$.

The above form assigns the identity element to $k-1$, in order to maximize our representation. We will also speak of the order of a group element, $|g|$, as the smallest power $n$ such that $g^{n}=e$. When looking for the canonical naming of a group, the first thing we do is to find the smallest order of an element in $G$. This number is the smallest prime number that divides $|G|$. We will assign $k-2$ to one of these. To know how we proceed further, we will illustrate by constructing and representing the groups of the first few orders.

We start with the trivial group of one object. The group $G_{0}$ is determined by the relation $* a(a)=a$. We have the trivial naming $a=0$ and the operation function $N_{* 0}$ is the one component function $P_{0}=N_{0}=2^{2^{1}+2^{2}}$. The canonical representation is

$$
G_{0}=2^{2^{2(0)+1}+2^{2\left(2^{2^{1}+2^{2}}+1\right)}}=2^{2^{1}+2^{2\left(2^{6}+1\right)}} .
$$

This number has $10^{19}$ decimal digits. It does not matter, how large it is, because we are able to easily manipulate and interpret these large numbers in terms of simple operations and functions for much smaller numbers. Simply said, we can work with these large numbers we are using.

Before continuing on to more groups, we clarify the use of a table notation, based on the fact that we are trying to represent a set of permutations. We will use the same notation of a column of arrows to represent a single permutation, but now we will ignore the arrows. For example, the permutation $(a, b)(c)(d)$ can be written as

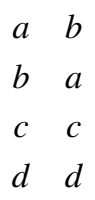

If we wish to represent several permutations of the same size, we can do this in a single rectangular grid. For this, we need to use one column as a pivot for the rest. For example, the set of permutations $\{(a, b)(c)(d),(a, b, c, d),(a)(c, b)(d)\}$ can be written as a single rectangular grid of $3+1$ columns. The first (left-most) column serves as pivot by which all other columns are defined. In general we would have a rectangular table. In this case, we have four objects and four columns so that we have a square table. The first column represents the permutation $(a, b)(c)(d)$, the second column represents the permutation $(a, b, c, d)$, and the third column is $(a)(b, c)(d)$.

$\begin{array}{llll}c & c & d & b \\ a & b & b & a \\ b & a & c & c \\ d & d & a & d\end{array}$


In the particular case of groups, the table is square and rows and columns do not repeat objects. Additionally, we need to have one column equal to the identity permutation, so we have the following convention. The left-most column will represent the identity permutation and we only need to write it once. The identity object will be in the upper left hand corner. The second column is representing the operation function of the second object in the first column. The third column represents the operation function of the third object in the first column. In general, if $a$ is the $k-t h$ object in the first column, then the operation function $* a$ is represented in the $k-t h$ column table. This simply means that we will write an operation in the usual table form, so that the following table has products such as $e * e=e, a * e=a, b * b=e, a * c=e$, and the like.

$\begin{array}{llll}e & a & b & c \\ a & b & c & e \\ b & c & e & a \\ c & e & a & b\end{array}$

In our process of finding representations and naming functions of groups, we will also need to verify the associative property holds. This is given by a simple rule on the table. Let $x$ be any object in a group $G$ of order $n$. We know $x$ appears in the table exactly $n$ times; once in each column/row. Each one of the positions where $x$ appears, gives us an expression for $x$ in terms of two objects; a factorization $x=y * z$. This simply means that given any fixed position, the object in that position is expressed in terms of the first object of that row and column. This form of writing the operation functions coincides with the multiplication table of the group. If $x$ is the $k-t h$ object in the first column, then the $k-t h$ column gives the function $* x$. Given a table representing a set of operation functions, the operation satisfies the associative property table if and only if $* x=* y \circ * z$, for every factorization $x=y * z$ of every $x \in G$. In the table above, we have $b=a * a$ so that we need to verify $* b=* a \circ * a$. To verify this is true, we have to verify $* b(g)=(* a \circ * a)(g)$ for every $g \in G$. To find $b * c=* b(c)$, we have the arrows $c \rightarrow_{* a} e \rightarrow_{* a} a$. We also have $b * a=* b(a)$ given by the arrows $a \rightarrow_{* a} b \rightarrow_{* a} c$. For another example, take the product $e=c * a$. This means we have to verify $* c \circ * a$ is the identity function. Let us find $(* c \circ * a)(b)$. We have the arrows $b \rightarrow_{* a} c \rightarrow_{* c} b$. We also have $(* c \circ * a)(a)$ given by $a \rightarrow_{* a} b \rightarrow_{* c} a$, etc.

We continue with the construction of groups, having in mind the above rules. Let us start with a group of two objects, so we list the objects. The second column gives the operation function of $g_{1}$, and since $* g_{1}(e)=g_{1}$,

e $g_{1}$

$g_{1}$

This is just another way of writing $e \rightarrow_{* g_{1}} g_{1}$. Furthermore, $g_{1}$ has an inverse $\neq e$, so we must have $g_{1} * g_{1}=e$,

$g_{1}$

$g_{1} \quad e$

We have the naming $e=1, g_{1}=0$, so we can rewrite our table as

$\begin{array}{ll}1 & 0 \\ 0 & 1\end{array}$

Let us find the representation of this group. Our group is an operation. This operation is a concrete function of two components. The first component is $*(1)=\mathbf{i d}$, that sends 1 to the identity function. The second component of our operation is $*\left(g_{1}\right)=(0,1)$, that sends the object 0 to the concrete permutation $(0,1)$. The canonical representation has two terms. 
The first term representing the first component is $2^{2^{2(1)+1}+2^{2}\left(2^{2^{3}+2^{4}}+2^{2^{1}+2^{2}}+1\right)}$. The second component is given by the expression

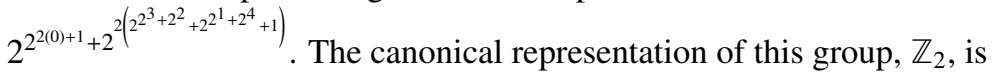

$$
\begin{aligned}
& G_{\mathbb{Z}_{2}}=2^{2^{2(1)+1}+2^{2\left(2^{2^{3}+2^{4}}+2^{2^{1}}+2^{2}+1\right)}}+2^{2^{2(0)+1}+2^{2\left(2^{2^{3}+2^{2}}+2^{2^{1}+2^{4}}+1\right)}} \\
& =2^{2^{3}+2^{2\left(2^{6}+2^{24}+1\right)}}+2^{2+2^{2\left(2^{18}+2^{12}+1\right)}}
\end{aligned}
$$

This number has somewhere around $10^{10^{\prime} 000,000}$ digits. If we wish to make a distinction, we say the terms are the numbers representing the arrows $x \rightarrow_{*} * x$. The upper terms are those representing arrows of the operation functions, we call them subterms. For example, $2^{2^{3}+2^{2}}$ is a subterm of the term $2^{2^{2(0)+1}+2^{2\left(2^{2^{3}}+2^{2}+2^{2^{1}}+2^{4}+1\right)}}$. We know terms are ordered pairs, in the sense they are elements of the set $\bigcup_{i}(i$,$) , defined at the beginning of section 4.1. Notice in the second equality, that subterms are$ also ordered pairs. For example, the subterm $2^{2^{3}+2^{2}}$ and the term $2^{3}+2^{2\left(2^{6}+2^{24}+1\right)}$ are both numbers of the form $2^{2 m+1}+2^{2(n+1)}$. They are both concrete arrows.

Why do we say $(8)$ is the canonical representation? The canonical representation is the maximum of the representations. In this case we have two possible representations, one for each naming function. If we had used the naming $e=0$ and $g_{1}=1$, we would have the representation

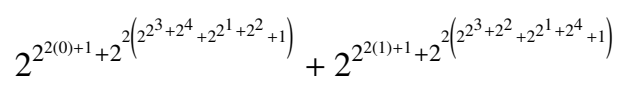

because now 0 is assigned to the identity function, while 1 is assigned the permutation $(0,1)$. This representation is smaller than the canonical representation above. The reader should understand why this is true, before moving on to the next examples. Remember, we will always assign the largest number of the naming, to the identity object because this maximizes our representation. We will see how to name the rest of the objects, to obtain the canonical representation.

\section{1 $|G|=3$}

Next we have the groups of three objects. We start with our table

$$
\begin{array}{ccc}
e & g_{1} & g_{2} \\
g_{1} & & \\
g_{2} & &
\end{array}
$$

The smallest order of the group is the smallest prime divisor of 3 , so that all three objects are of order 3 . This means $g_{1} * g_{1} \neq e$. Since $g_{1}$ is not the identity element, we also know $g_{1} * g_{1} \neq g_{1}$. Therefore, $g_{1} * g_{1}=g_{2}$, and the rest of the table is determined. This is the group $\mathbb{Z}_{3}$.

$$
\begin{array}{ccc}
e & g_{1} & g_{2} \\
g_{1} & g_{2} & e \\
g_{2} & e & g_{1}
\end{array}
$$

The canonical representation has the naming $e=2$, and we will place the objects in order, top to bottom, in the first column. If we make $g_{1}=1$ and $g_{2}=0$, we have the numeric table 


$$
\begin{array}{lll}
2 & 1 & 0 \\
1 & 0 & 2 \\
0 & 2 & 1
\end{array}
$$

If we change the naming to $g_{1}=0$ and $g_{2}=1$ we get the same numeric table. Therefore, $g_{1}, g_{2}$ are equivalent objects in the group. Given any of the two naming functions with $e=2$, we have the numerical table above, so the canonical representation is given by either of these naming functions. The representation is a concrete function of three components. The first component is the ordered pair that assigns 2 to the concrete permutation, (1)(2)(3). This ordered pair is represented by the number

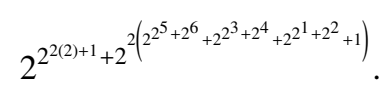

The second component assigns 1 to the concrete permutation $(2,1,0)$. This is given by

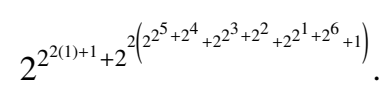

The object 0 is assigned the permutation $(2,0,1)$, so our third term is

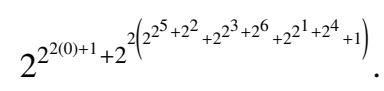

We finally have the canonical representation,

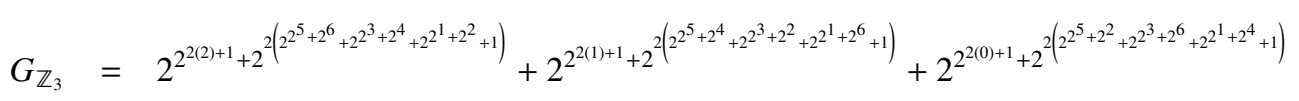

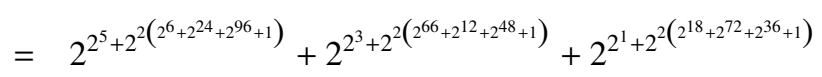

This number is approximately as large as $10^{10^{10^{28}}}$.

\section{2 $|G|=4$}

Klein 4-Group. We start with a set $\left\{e, g_{1}, g_{2}, g_{3}\right\}$, where we use $e$ to denote the identity element. There is at least one object with order equal to the smallest prime divisor of 4 . We suppose $g_{1} * g_{1}=e$, without loss of generality.

$$
\begin{array}{cccc}
e & g_{1} & g_{2} & g_{3} \\
g_{1} & e & g_{3} & g_{2} \\
g_{2} & g_{3} & & \\
g_{3} & g_{2} & &
\end{array}
$$

Now we can make $g_{2} * g_{2}=e$ or $g_{2} * g_{2}=g_{1}$. In the first case, we have determined the Klein four-group, $K(4)$. 


$\begin{array}{cccc}e & g_{1} & g_{2} & g_{3} \\ g_{1} & e & g_{3} & g_{2} \\ g_{2} & g_{3} & e & g_{1} \\ g_{3} & g_{2} & g_{1} & e\end{array}$

In this group, the objects $g_{1}, g_{2}, g_{3}$ are equivalent. Any naming function with $e=3$, gives the numeric table

$\begin{array}{llll}3 & 2 & 1 & 0 \\ 2 & 3 & 0 & 1 \\ 1 & 0 & 3 & 2 \\ 0 & 1 & 2 & 3\end{array}$

with canonical representation

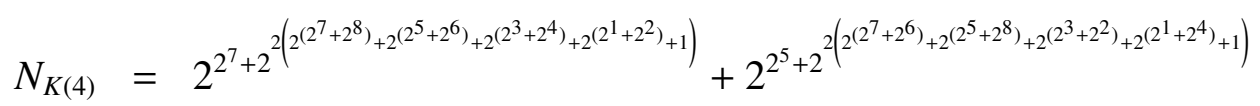

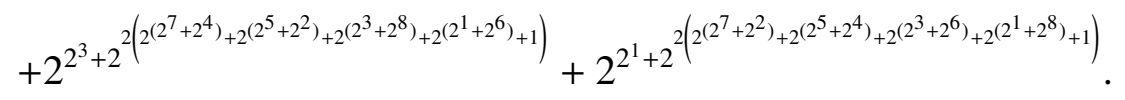

The first term is the component that send 3 to the identity function, while the second term is the component that sends 2 to the permutation $(0,1)(2,3)$, etc. We bring something new to attention. The group $K(4)$ is defined by $|G|=4$, and a minimum set of independent equations. Thus, any group $|G|=4$ such that $e=g_{1}^{2}=g_{2}^{2}$, for $g_{1}, g_{2} \in G$, is isomorphic to $K(4)$.

Cyclic group $\mathbb{Z}_{4}$. Going back to table 99, consider the second case, $g_{2} * g_{2}=g_{1}$. This determines the table

$$
\begin{array}{cccc}
e & g_{1} & g_{2} & g_{3} \\
g_{1} & e & g_{3} & g_{2} \\
g_{2} & g_{3} & g_{1} & e \\
g_{3} & g_{2} & e & g_{1}
\end{array}
$$

This is the cyclic group, $\mathbb{Z}_{4}$. This is our first non trivial canonical naming. To maximize our representation, we are careful assigning the values. Let us recall the numeric form of a group, and how to compare two group representations. The representation will be of the form

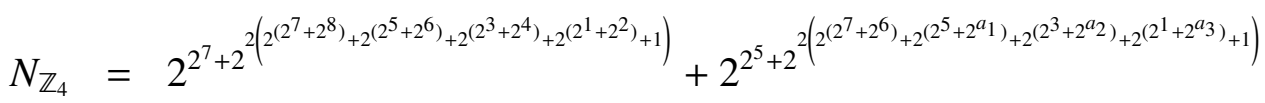

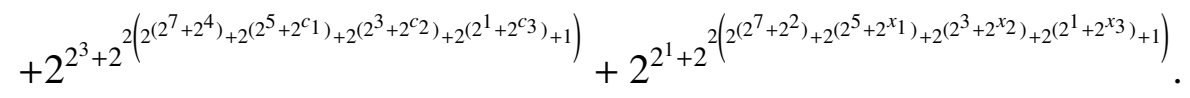

We know there is exactly one object $g$ such that $(2,3)=2^{2^{5}+2^{8}} \in * g$. If we have $2^{2^{5}+2^{8}}$ in the second term, this would mean $(2,3) \in * 2$, which is another way of saying $2 * 2=3$. We have only one object, $g_{1}$, that satisfies this, so we assign to it the next value of the naming function, $g_{1}=2$. Giving the naming function $\rho: e=3, g_{1}=2$, we have 


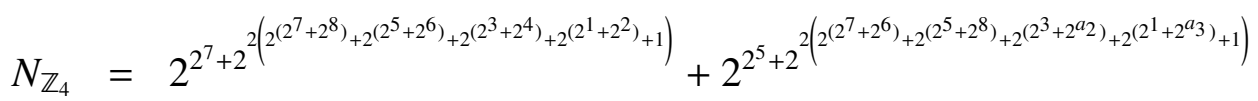

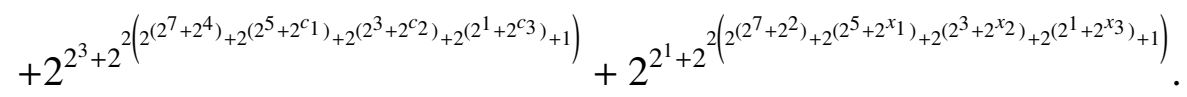

or, equivalently, in table form,

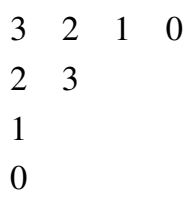

The first term will be the same in any naming with $e=3$. Also, the first subterm of every term will be the same. For the naming $\rho$, we have the subterm $2^{2^{5}+2^{8}}$ in the second term, representing $2 * 2=3$. This maximizes the representation because the second term also contains $2^{2^{7}+2^{6}}$ representing $2 * 3=2$. Then, we have

$\begin{array}{llll}3 & 2 & 1 & 0 \\ 2 & 3 & 0 & 1 \\ 1 & 0 & & \\ 0 & 1 & & \end{array}$

We know that both objects $\left|g_{2}\right|=4$, so the rest of the table is determined

$\begin{array}{llll}3 & 2 & 1 & 0 \\ 2 & 3 & 0 & 1 \\ 1 & 0 & 2 & 3 \\ 0 & 1 & 3 & 2\end{array}$

A group $|G|=4$ satisfying the equations $g_{1}^{2}=e$ and $g_{2}^{2}=g_{1}$, for $g_{1}, g_{2}, \in G$, is isomorphic to $\mathbb{Z}_{4}$. The canonical naming is $\rho: e=3, g_{1}=2, g_{2}=1, g_{3}=0$, and $g_{2}, g_{3}$ are equivalent. The canonical representation is

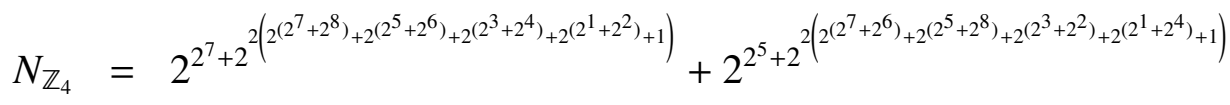

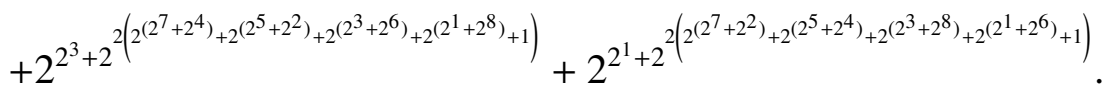

To compare the two groups $K(4)$ and $\mathbb{Z}_{4}$ we must find the number with the largest operation function that is not in both groups. The group that contains that function, will be the larger of the two. We have the inequality $N_{\mathbb{Z}_{4}}<N_{K(4)}$, of canonical representations of $\mathbb{Z}_{4}$ and $K(4)$. As we would expect, the cyclic group has the smallest representation, just as the one cycle permutation $(a, b, c, d)$ has smaller representation than $(a, b)(c, d)$. 


\section{3 $|G|=5$}

We know the smallest order of any object has to be equal to the smallest prime divisor of 5 . Therefore, all non trivial objects are of order 5. Without loss of generality we have

$\begin{array}{lllll}e & g_{1} & g_{2} & g_{3} & g_{4} \\ g_{1} & g_{2} & & & \\ g_{2} & g_{3} & & & \\ g_{3} & g_{4} & & & \\ g_{4} & e & & & \end{array}$

Now, we have to use associativity. We wish to find the operation function of $g_{2}$, and it will be placed in the second column. But, we know $g_{2}=g_{1} * g_{1}$, so that we must have $* g_{2}=* g_{1} \circ * g_{1}$. This means $* g_{2}\left(g_{1}\right)$ is found by $g_{1} \rightarrow_{* g_{1}} g_{2} \rightarrow_{* g_{1}} g_{3}$. Then we also have $* g_{2}\left(* g_{2}\right)$ is found by $g_{2} \rightarrow_{* g_{1}} g_{3} \rightarrow_{* g_{1}} g_{4}$, etc.

$\begin{array}{lllll}e & g_{1} & g_{2} & g_{3} & g_{4} \\ g_{1} & g_{2} & g_{3} & & \\ g_{2} & g_{3} & g_{4} & & \\ g_{3} & g_{4} & g_{5} & & \\ g_{4} & e & g_{1} & & \end{array}$

We do the same with the column of $g_{3}=g_{1} * g_{2}$ and $g_{4}=g_{1} * g_{3}$, so that $* g_{3}=* g_{1} \circ * g_{2}$ and $* g_{4}=* g_{1} \circ * g_{3}$. For example, $g_{3} * g_{1}=* g_{3}\left(g_{1}\right)$ is given by the arrows $g_{1} \rightarrow_{* g_{2}} g_{3} \rightarrow_{* g_{1}} g_{4}$, etc.

$\begin{array}{ccccc}e & g_{1} & g_{2} & g_{3} & g_{4} \\ g_{1} & g_{2} & g_{3} & g_{4} & e \\ g_{2} & g_{3} & g_{4} & e & g_{1} \\ g_{3} & g_{4} & e & g_{1} & g_{2} \\ g_{4} & e & g_{1} & g_{2} & g_{3}\end{array}$

If we had chosen any non trivial object, $\neq g_{1}$, at the beginning, we would end up with the same table. All we would be doing is changing the symbols with respect to positions, but the structure is the same; we chose $g_{1}$ without loss of generality. Then, we had to choose a second object for $g_{1} * g_{1}$; the only condition is that $g_{2} \neq e, g_{1}$. Then we have to select an object $g_{3}$ with the only restriction it be different than $\neq e, g_{1}, g_{2}$ so we took $g_{3}=g_{1} * g_{2}$. Finally, we had to choose an object for $g_{1} * g_{3}$ and it had to be different than $e, g_{1}, g_{2}, g_{3}$ so we trivially choose $g_{4}$. No other restrictions were imposed during this process so that it does not depend on our initial choice, nor the second, third, etc. We simply have to take new elements, without observing any other relations. The group is defined solely by the number of objects. In the next examples, we will have to satisfy certain restrictions on each step of our process, to find the canonical representation and naming. The numerical table for this group is given by the naming function, $\rho: e=4, g_{1}=3, g_{2}=2, g_{3}=1, g_{4}=0$, where all the objects are equivalent. This means we can take other canonical naming functions, for example, $\rho: e=4, g_{4}=3, g_{3}=2, g_{2}=1, g_{1}=0$. The numeric table is 


$\begin{array}{lllll}4 & 3 & 2 & 1 & 0 \\ 3 & 2 & 1 & 0 & 4 \\ 2 & 1 & 0 & 4 & 3 \\ 1 & 0 & 4 & 3 & 2 \\ 0 & 4 & 3 & 2 & 1\end{array}$

and the canonical representation is

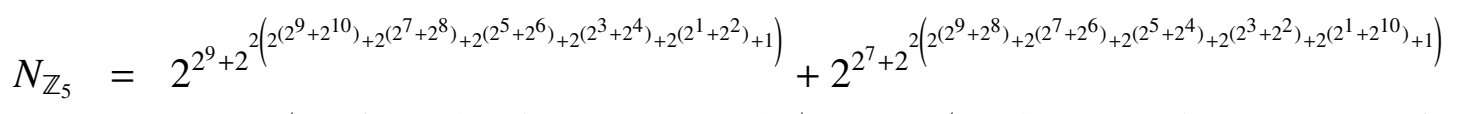

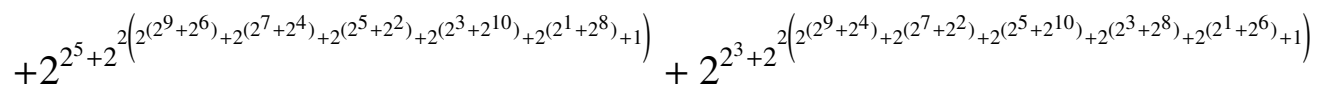

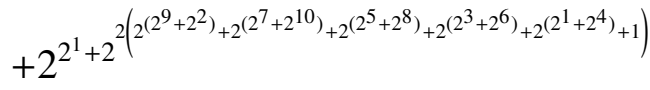

\section{$4.4|G|=6$}

We move on to groups of six objects, and things start to be clearer. We begin as usual with the list of objects.

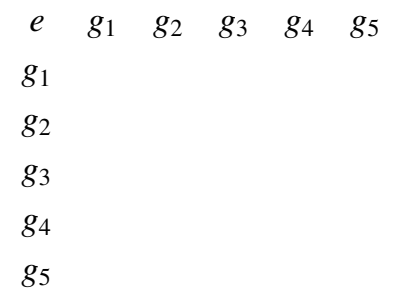

We know we will have at least one element of order equal to the smallest prime divisor of 6 . That is to say, There is at least one object of order 2. Since 3 is a prime divisor of 6, we also know our group has at least one object of order 3. In fact, there has to be a multiple of $\phi(3)=2$ many objects of order 3 . Therefore, we can consider groups of six objects with two, or four, objects of order 3 . Let us consider the case where we have two objects of order 3 , and three objects of order 2 . We can suppose, without loss of generality, $g_{1}^{2}=g_{2}$ and $g_{1} * g_{2}=e$.

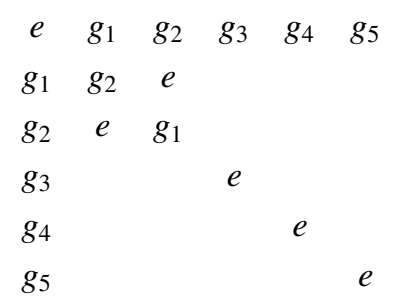

Then we make $g_{1} * g_{3}=g_{4}$, without loss of generality. It is trivial to find the column of $g_{1}$ because $\left|g_{1}\right|=3$. Then we find the function $* g_{2}$ by means of the composition $* g_{1} \circ * g_{1}$. 


$\begin{array}{llllll}e & g_{1} & g_{2} & g_{3} & g_{4} & g_{5} \\ g_{1} & g_{2} & e & & & \\ g_{2} & e & g_{1} & & & \\ g_{3} & g_{4} & g_{5} & e & & \\ g_{4} & g_{5} & g_{3} & & e & \\ g_{5} & g_{3} & g_{4} & & & e\end{array}$

Then, we use $\left|g_{3}\right|=2$ to find

$\begin{array}{llllll}e & g_{1} & g_{2} & g_{3} & g_{4} & g_{5} \\ g_{1} & g_{2} & e & & & \\ g_{2} & e & g_{1} & & & \\ g_{3} & g_{4} & g_{5} & e & & \\ g_{4} & g_{5} & g_{3} & g_{2} & & \\ g_{5} & g_{3} & g_{4} & g_{1} & & \end{array}$

Again, we use $\left|g_{3}\right|=2$, now to find

$\begin{array}{llllll}e & g_{1} & g_{2} & g_{3} & g_{4} & g_{5} \\ g_{1} & g_{2} & e & g_{5} & & \\ g_{2} & e & g_{1} & g_{4} & & \\ g_{3} & g_{4} & g_{5} & e & & \\ g_{4} & g_{5} & g_{3} & g_{2} & & \\ g_{5} & g_{3} & g_{4} & g_{1} & & \end{array}$

It is trivial to find the columns of $g_{4}, g_{5}$ in terms of the rest of the columns, using associativity as usual.

$\begin{array}{cccccc}e & g_{1} & g_{2} & g_{3} & g_{4} & g_{5} \\ g_{1} & g_{2} & e & g_{5} & g_{3} & g_{4} \\ g_{2} & e & g_{1} & g_{4} & g_{5} & g_{3} \\ g_{3} & g_{4} & g_{5} & e & g_{1} & g_{2} \\ g_{4} & g_{5} & g_{3} & g_{2} & e & g_{1} \\ g_{5} & g_{3} & g_{4} & g_{1} & g_{2} & e\end{array}$

This is the symmetry group $\Delta_{3}$. It is determined by the set of equations

$$
\begin{aligned}
e & =g_{1}^{2} \\
g_{3} & =g_{2}^{2} \\
e & =g_{2} * g_{3} \\
g_{4} & =g_{1} * g_{2} \\
g_{5} & =g_{2} * g_{1} .
\end{aligned}
$$


The last two equations can be replaced by $g_{1} * g_{2} \neq g_{2} * g_{1}$. Now we consider the case with four objects of order 4 , and one object of order 2. We begin with

$\begin{array}{cccccc}e & g_{1} & g_{2} & g_{3} & g_{4} & g_{5} \\ g_{1} & e & & & & \\ g_{2} & & g_{3} & e & & \\ g_{3} & & e & g_{2} & & \\ g_{4} & & & & & \\ g_{5} & & & & & \end{array}$

We make $g_{1} * g_{2}=g_{4}$, without loss of generality,

$\begin{array}{cccccc}e & g_{1} & g_{2} & g_{3} & g_{4} & g_{5} \\ g_{1} & e & & & & \\ g_{2} & g_{4} & g_{3} & e & & \\ g_{3} & g_{5} & e & g_{2} & & \\ g_{4} & & & & & \\ g_{5} & & & & & \end{array}$

Using $\left|g_{1}\right|=2$, we have

$\begin{array}{llllll}e & g_{1} & g_{2} & g_{3} & g_{4} & g_{5} \\ g_{1} & e & & & & \\ g_{2} & g_{4} & g_{3} & e & & \\ g_{3} & g_{5} & e & g_{2} & & \\ g_{4} & g_{2} & & & & \\ g_{5} & g_{3} & & & & \end{array}$

Now use $\left|g_{2}\right|=\left|g_{3}\right|=3$ to find

$\begin{array}{llllll}e & g_{1} & g_{2} & g_{3} & g_{4} & g_{5} \\ g_{1} & e & & & & \\ g_{2} & g_{4} & g_{3} & e & g_{5} & g_{1} \\ g_{3} & g_{5} & e & g_{2} & g_{1} & g_{4} \\ g_{4} & g_{2} & & & & \\ g_{5} & g_{3} & & & & \end{array}$

We are considering $\left|g_{4}\right|=\left|g_{5}\right| \neq 2$, so that our only option is $g_{4}^{2}=g_{3}$ and $g_{5}^{2}=g_{2}$. 


$\begin{array}{cccccc}e & g_{1} & g_{2} & g_{3} & g_{4} & g_{5} \\ g_{1} & e & & & & \\ g_{2} & g_{4} & g_{3} & e & g_{5} & g_{1} \\ g_{3} & g_{5} & e & g_{2} & g_{1} & g_{4} \\ g_{4} & g_{2} & & & g_{3} & \\ g_{5} & g_{3} & & & & g_{2}\end{array}$

However, it is easy to see that $\left|g_{4}\right|=\left|g_{5}\right|=6$. We conclude there is no group $|G|=4$ with four objects of order 2 . The table is determined and we obtain the cyclic group.

$\begin{array}{cccccc}e & g_{1} & g_{2} & g_{3} & g_{4} & g_{5} \\ g_{1} & e & g_{4} & g_{5} & g_{2} & g_{3} \\ g_{2} & g_{4} & g_{3} & e & g_{5} & g_{1} \\ g_{3} & g_{5} & e & g_{2} & g_{1} & g_{4} \\ g_{4} & g_{2} & g_{5} & g_{1} & g_{3} & e \\ g_{5} & g_{3} & g_{1} & g_{4} & e & g_{2}\end{array}$

This the cyclic group $\mathbb{Z}_{6}$ is determined by $|G|=6$ and objects $g_{1}, g_{2}, g_{3}$ such that

$$
\begin{aligned}
e & =g_{1}^{2} \\
g_{3} & =g_{2}^{2} \\
e & =g_{2} * g_{3} \\
g_{4} & =g_{1} * g_{2}=g_{2} * g_{1} .
\end{aligned}
$$

The first of these equations tells us we have one object of order 2, the second and third equations tell us we have two objects of order 3 . The last equation requires the second order object to commute with one of the non trivial elements of order 3 . Any group of six objects satisfying these equations is isomorphic to $\mathbb{Z}_{6}$.

The tables (11) and 107 have given us the groups of six objects, $\Delta_{3}$ and $\mathbb{Z}_{6}$. Now, we would like to find the canonical naming and representation for both of these. Again, we will have the cyclic group, $\mathbb{Z}_{6}$, represented by the smallest number. We will also observe the block form of these groups.

Symmetry group $\Delta_{3}$. For reference, we repeat table (10), below.

$\begin{array}{cccccc}e & g_{1} & g_{2} & g_{3} & g_{4} & g_{5} \\ g_{1} & e & g_{5} & g_{4} & g_{3} & g_{2} \\ g_{2} & g_{4} & g_{3} & e & g_{5} & g_{1} \\ g_{3} & g_{5} & e & g_{2} & g_{1} & g_{4} \\ g_{4} & g_{2} & g_{1} & g_{5} & e & g_{3} \\ g_{5} & g_{3} & g_{4} & g_{1} & g_{2} & e\end{array}$


We will use letters $a, b, c, \ldots$ and $x_{1}, x_{2}, x_{3}, \ldots$ as auxiliary variables in finding our canonical naming. We start using this now, and in larger example it will be more useful. We know we have to start with naming $e=5$. One of our three objects of second order, call it $a$, will be assigned the naming value $a=4$. Our first observation is that we have three objects of second order, so our first task is to find which of these will be assigned the value 4 . We are going to use new letters for simplicity of notation. So far we have part of the table,

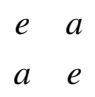

which numerically is

$$
\begin{array}{ll}
5 & 4 \\
4 & 5
\end{array}
$$

Intuitively, we are trying to assign the larger numbers by giving priority to the left-most columns. Within a column we are giving priority to the objects of upper rows. Let us add an object $b \neq a$. Whatever object we may choose for $b$, we have a fourth object, $a * b=x_{1}$.

$\begin{array}{llll}e & a & b & x_{1} \\ a & e & & \\ b & x_{1} & & \\ x_{1} & b & & \end{array}$

In order to maximize our representation we name $b=3$ and $a * b=x_{1}=2$. That way, we have the numeric table

$\begin{array}{llll}5 & 4 & 3 & 2 \\ 4 & 5 & & \\ 3 & 2 & & \\ 2 & 3 & & \end{array}$

We know, from the table defining $\Delta_{3}$, that there is no object $x_{1}$ that commutes with $a$. Therefore, we need new objects $c=b * a$ and $x_{2}=a * c$,

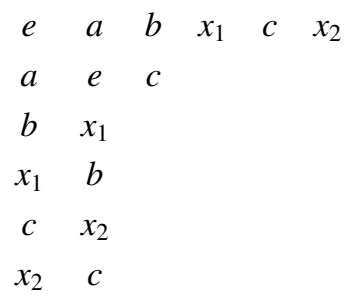

If we make $|b|=2$, we are maximizing our representation. We choose one of the two remaining objects of second order, to assign the name $b=2$. This gives the table 


$\begin{array}{cccccc}e & a & b & x_{1} & c & x_{2} \\ a & e & c & & & \\ b & x_{1} & e & & & \\ x_{1} & b & x_{2} & & & \\ c & x_{2} & a & & & \\ x_{2} & c & x_{1} & & & \end{array}$

The rest of the table is determined using associativity as before. For example, the column of $x_{1}$ is given by the composition $* a \circ * b$. Let us take $a=g_{4}$ and $b=g_{5}$, so that $x_{1}=g_{2}$. Then we have $c=g_{3}$ and $x_{2}=g_{1}$. In numeric form, we have

$\begin{array}{llllll}5 & 4 & 3 & 2 & 1 & 0 \\ 4 & 5 & 1 & 0 & 3 & 2 \\ 3 & 2 & 5 & 4 & 0 & 1 \\ 2 & 3 & 0 & 1 & 5 & 4 \\ 1 & 0 & 4 & 5 & 2 & 3 \\ 0 & 1 & 2 & 3 & 4 & 5\end{array}$

To obtain a canonical naming function, make $a=4, b=3$ for any two objects of order 2, then make $a * b=x_{1}=2$ and $b * a=c=1$ and $a * c=x_{2}=0$. Obviously, $g_{1}, g_{4}, g_{5}$ are equivalent objects, as are $g_{2}, g_{3}$. We have block form, but the blocks are not cosets of a normal subgroup (even though $\Delta_{6}$ has a normal subgroup). We have a table of four $3 \times 3$ blocks, and there are two types of blocks. The first type of block has objects in $A=\{1,2,3,4,5\}$ while the second type of block has objects in $B=\{0,1,2,3,4\}$. We have blocks $A_{1}$ and $A_{2}$ in the upper left corner and lower right corner, respectively. We have blocks $B_{1}$ and $B_{2}$ in the upper right hand and lower left hand, respectively. In this case, we have a normal subgroup, but it is not apparent in the canonical naming table. The normal subgroup is $N=\left\{e=5, g_{2}=2, g_{3}=1\right\}$. We can write the canonical table in such a manner that $N$ will be trivially seen. The only thing that we have to do is change the rows and columns, appropriately.

This group has canonical representation

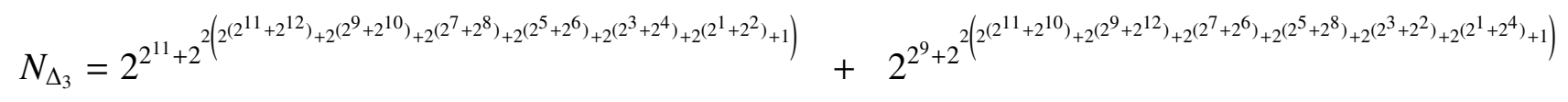

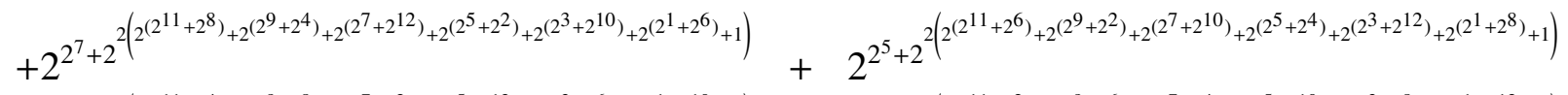

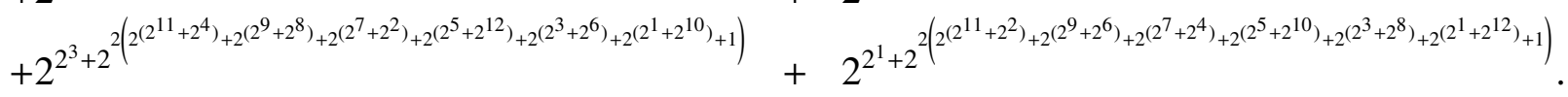

Cyclic Group $\mathbb{Z}_{6}$. The table (11) is given again, for reference.

$\begin{array}{cccccc}e & g_{1} & g_{2} & g_{3} & g_{4} & g_{5} \\ g_{1} & e & g_{4} & g_{5} & g_{2} & g_{3} \\ g_{2} & g_{4} & g_{3} & e & g_{5} & g_{1} \\ g_{3} & g_{5} & e & g_{2} & g_{1} & g_{4} \\ g_{4} & g_{2} & g_{5} & g_{1} & g_{3} & e \\ g_{5} & g_{3} & g_{1} & g_{4} & e & g_{2}\end{array}$


We use $a, b, c, \ldots$ and $x_{1}, x_{2}, x_{3}, \ldots$ as auxiliary variables in finding our canonical naming. We with start naming $e=5$ and since we only have one object of order 2 we make $g_{1}=a=4$. We are going to use new letters only for simplicity of notation. We will use $a=g_{1}$. So far we have part of the table,

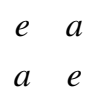

Let us add an object $b \neq g_{1}$. Whatever object we may choose for $b$, we have another object $a * b=x_{1}$.

$\begin{array}{cccc}e & a & b & x_{1} \\ a & e & & \\ b & x_{1} & & \\ x_{1} & b & & \end{array}$

In order to maximize our representation we name $b=3$ and $a * b=x_{1}=2$. That way, we have the numeric table

$\begin{array}{llll}5 & 4 & 3 & 2 \\ 4 & 5 & & \\ 3 & 2 & & \\ 2 & 3 & & \end{array}$

This maximizes our representation. We still do not know what object of the group will be assigned to $b=3$. But we know, from the table, $a=g_{1}$ commutes with any object. We have the numeric table

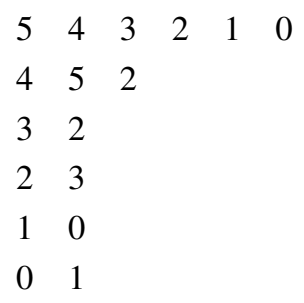

Notice, $g_{1}$ commutes with $g_{2}, g_{3}, g_{4}, g_{5}$, so our options are not limited. We will eliminate possible naming functions, by focusing on the larger ones. So far, we have four candidate naming functions. Each column is a different naming function,

$\begin{array}{cccc}e=5 & e=5 & e=5 & e=5 \\ g_{1}=a=4 & g_{1}=a=4 & g_{1}=a=4 & g_{1}=a=4 \\ g_{2}=b=3 & g_{3}=b=3 & g_{4}=b=3 & g_{5}=b=3 \\ g_{4}=x_{1}=2 & g_{5}=x_{1}=2 & g_{2}=x_{1}=2 & g_{3}=x_{1}=2\end{array}$

Each of these naming functions gives the table (12). We can easily see, in table (11), none of these eight naming functions has $b^{2} \in\{e, a\}$. Observe $g_{2}^{2}, g_{3}^{2}, g_{4}^{2}, g_{5}^{2} \neq e, a$. Therefore, we must have $b^{2}=c=1$, for some new object $c \neq e, b, x_{1}$. Of course, we also have the new object $a * c=x_{2}=0$. 


$\begin{array}{cccccc}e & a & b & x_{1} & c & x_{2} \\ a & e & x_{1} & b & & \\ b & x_{1} & c & & & \\ x_{1} & b & & & & \\ c & x_{2} & & & \\ x_{2} & c & & & & \end{array}$

None of our candidate naming functions satisfy $b * x_{1} \in\{e, a\}$. For example, the first naming function has $b * x_{1}=g_{2} * g_{4}=g_{5}$, and the second naming function has $b * x_{1}=g_{3} * g_{5}=g_{4}$, etc. This implies $b * x_{1}=x_{2}$,

$\begin{array}{cccccc}e & a & b & x_{1} & c & x_{2} \\ a & e & x_{1} & b & & \\ b & x_{1} & c & & & \\ x_{1} & b & x_{2} & & & \\ c & x_{2} & & & \\ x_{2} & c & & & & \end{array}$

We complete our possible naming functions, using $c=b^{2}$ and $x_{2}=a * c$.

$\begin{array}{cccc}e=5 & e=5 & e=5 & e=5 \\ g_{1}=a=4 & g_{1}=a=4 & g_{1}=a=4 & g_{1}=a=4 \\ g_{2}=b=3 & g_{3}=b=3 & g_{4}=b=3 & g_{5}=b=3 \\ g_{4}=x_{1}=2 & g_{5}=x_{1}=2 & g_{2}=x_{1}=2 & g_{3}=x_{1}=2 \\ g_{g 3}=c=1 & g_{2}=c=1 & g_{3}=c=1 & g_{2}=c=1 \\ g_{5}=x_{2}=0 & g_{4}=x_{2}=0 & g_{5}=x_{2}=0 & g_{4}=x_{2}=0\end{array}$

We see $g_{2}, g_{3}, g_{4}, g_{5}$ are equivalent objects. Any of these naming functions gives

$\begin{array}{cccccc}e & a & b & x_{1} & c & x_{2} \\ a & e & x_{1} & b & & \\ b & x_{1} & c & & & \\ x_{1} & b & x_{2} & & & \\ c & x_{2} & e & & \\ x_{2} & c & a & & & \end{array}$

The rest of the table is determined, and we still have the same possible naming functions. But, now we know the complete naming function, since we found we have to make $b^{2}=c=1$ and $a * c=x_{2}=0$ to maximize the representation. We have determined all the names we need and the canonical naming functions are given above. This provides the numerical table 


$\begin{array}{llllll}5 & 4 & 3 & 2 & 1 & 0 \\ 4 & 5 & 2 & 3 & 0 & 1 \\ 3 & 2 & 1 & 0 & 5 & 4 \\ 2 & 3 & 0 & 1 & 4 & 5 \\ 1 & 0 & 5 & 4 & 3 & 2 \\ 0 & 1 & 4 & 5 & 2 & 3\end{array}$

The $2 \times 2$ block on the upper left hand corner is the normal subgroup $N=\mathbb{Z}_{2}$. The table is made up of nine $2 \times 2$ blocks that are the cosets $N, b N$ and $b^{2} N$, for $b \in\left\{g_{2}, g_{3}\right\}$. These coset blocks form the group $\mathbb{Z}_{3}$. The canonical table above is written as

$$
\begin{array}{ccc}
N & b N & b^{2} N \\
b N & b^{2} N & N \\
b^{2} N & N & b N
\end{array}
$$

The canonical naming table gives us the additional information that $\mathbb{Z}_{6} / \mathbb{Z}_{2}=\mathbb{Z}_{3}$. These canonical representation of the cyclic group is

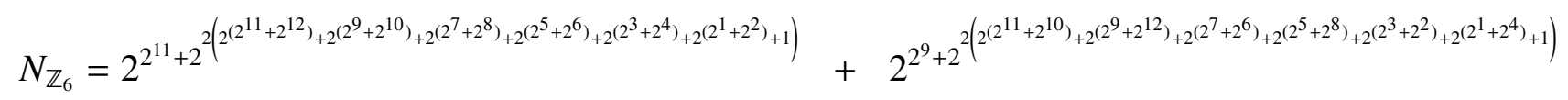

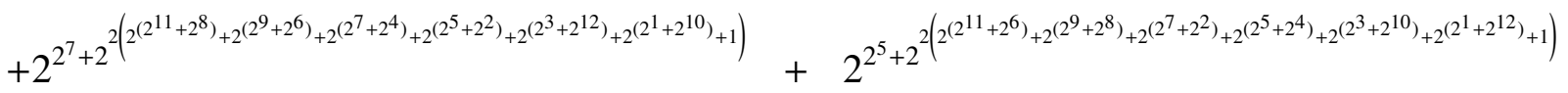

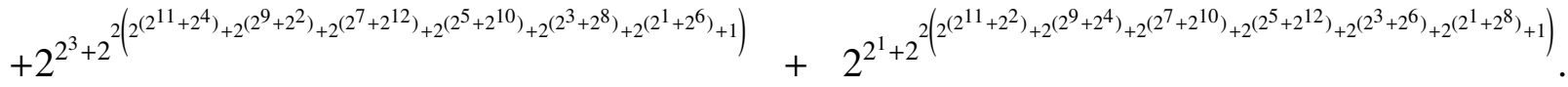

Up to this point, we have not had any difficulty in finding the canonical naming and representation of the smaller groups. So far we know that the first groups are ordered

$$
\begin{aligned}
G_{0} & =\mathbb{Z}_{1} \\
G_{1} & =\mathbb{Z}_{2} \\
G_{2} & =\mathbb{Z}_{3} \\
G_{3} & =\mathbb{Z}_{4} \\
G_{4} & =K(4) \\
G_{5} & =\mathbb{Z}_{5} \\
G_{6} & =\mathbb{Z}_{6} \\
G_{7} & =\Delta_{3}
\end{aligned}
$$

It may seem somewhat intuitive to assign numerical values to the elements of the group from this example, it is not completely clear that this can be carried on in general, for any finite group. Thus, it is possible that the only way of finding the canonical representation of some finite group is through calculating all possible representations. Of course, in certain cases we will be able to have optimized algorithms, but the general solution of finding the canonical representation may not be simple. 
This analysis is left for future work. Perhaps what is hardest is finding all groups of a fixed order, $|G|=n$. Still, we are able to easily find all possible groups for these and the next few orders. By now we know how to find the canonical table and representation of $\mathbb{Z}_{7}$. Of course, all the non trivial objects are equivalent.

$$
G_{8}=\mathbb{Z}_{7}
$$

\section{$4.5|G|=8$}

Let us find groups of eight objects. We know The possible orders of the objects are the divisors of 8 . Particularly, we have at least one object of order 2 , we can have $2 i$ objects of order 4 and we can have $4 j$ objects of order 8 . We will find all groups of eight objects. We will provide each group found with a canonical naming function given in the numeric table, the minimal independent set of equations that defines the group, and the canonical representation. Then, we will compare the canonical representations of these groups to find $G_{9}<G_{10}<G_{11}<G_{12}<G_{13}$.

Direct Product $\mathbb{Z}_{2} \oplus \mathbb{Z}_{2} \oplus \mathbb{Z}_{2}$. Let us take the simplest case first, and then it will be clear how we can complicate things little by little, so that we find all possible groups of order 8 . The simplest case is to consider all objects of order 2 (we make $i=j=0$ ). Additionally, we will suppose they all commute.

$\begin{array}{cccccccc}e & a & b & x_{1} & c & x_{2} & d & x_{3} \\ a & e & x_{1} & b & x_{2} & c & x_{3} & d \\ b & x_{1} & e & & & & & \\ x_{1} & b & & e & & & & \\ c & x_{2} & & & e & & & \\ x_{2} & c & & & & e & & \\ d & x_{3} & & & & & e & \\ x_{3} & d & & & & & & e\end{array}$

Since $|a|=2$, we know $b * x_{1}=a$ and commutativity gives $x_{1} * b=a$. In the same way, $a=c * x_{2}=x_{2} * c$, etc.

$\begin{array}{cccccccc}e & a & b & x_{1} & c & x_{2} & d & x_{3} \\ a & e & x_{1} & b & x_{2} & c & x_{3} & d \\ b & x_{1} & e & a & & & & \\ x_{1} & b & a & e & & & & \\ c & x_{2} & & & e & a & & \\ x_{2} & c & & & a & e & & \\ d & x_{3} & & & & & e & a \\ x_{3} & d & & & & & a & e\end{array}$

Next, we know $b * c \notin\left\{e, a, b, c, x_{1}, x_{2}\right\}$, and we suppose without loss of generality, $b * c=d$. This determines the rest of the column of $b$. Then we use $b * c=c * b$ and $b * d=d * b$ to find the third row. Now we can find the fourth column and fourth row using the associative property. For example, we use $d * b=c$ to find $c * x_{1}=d *\left(b * x_{1}\right)=d * a=x_{3}$. Finally, we use $x_{1} * x_{2}=d$ to find $d * c=x_{1} *\left(x_{2} * c\right)=x_{1} * a=b$ 


$\begin{array}{cccccccc}e & a & b & x_{1} & c & x_{2} & d & x_{3} \\ a & e & x_{1} & b & x_{2} & c & x_{3} & d \\ b & x_{1} & e & a & d & x_{3} & c & x_{2} \\ x_{1} & b & a & e & x_{3} & d & x_{2} & c \\ c & x_{2} & d & x_{3} & e & a & b & x_{1} \\ x_{2} & c & x_{3} & d & a & e & x_{1} & b \\ d & x_{3} & c & x_{2} & b & x_{1} & e & a \\ x_{3} & d & x_{2} & c & x_{1} & b & a & e\end{array}$

This determines the group $\mathbb{Z}_{2}^{3}=\mathbb{Z}_{2} \oplus \mathbb{Z}_{2} \oplus \mathbb{Z}_{2}$. Again, we have the special block form of cosets of $N=\mathbb{Z}_{2}$. The expression $\mathbb{Z}_{8} / N=\mathbb{Z}_{4}$ is given in the table, because we have sixteen $2 \times 2$ blocks, $N, b N, c N, d N$, forming $\mathbb{Z}_{4}$.

$\begin{array}{cccc}N & b N & c N & d N \\ b N & N & d N & c N \\ c N & d N & N & b N \\ d N & c N & b N & N\end{array}$

This coset table, is itself an expression of quotient groups. It expresses $\mathbb{Z}_{4} / \mathbb{Z}_{2}=\mathbb{Z}_{2}$, because it is a table of the cosets of the normal group $N_{2}=\{N, b N\}<\mathbb{Z}_{4}$. We have a third expression of group quotients, going back to table (13). It simultaneously shows $\mathbb{Z}_{8} / \mathbb{Z}_{4}=\mathbb{Z}_{2}$ because we have four $4 \times 4$ blocks forming $\mathbb{Z}_{2} ;$ these blocks are the cosets of $N_{3}=\mathbb{Z}_{4}$,

$$
\begin{array}{cc}
N_{3} & c N_{3} \\
c N_{3} & N_{3}
\end{array}
$$

To define this group we need four objects of order 2; these are $a, b, c, d$ (the remaining objects are order two by consequence of this). We also need for $a$ to commute with $b, c, d$. We also need $b$ to commute with $c, d$.

$$
\begin{array}{ll}
e=a^{2}=b^{2}=c^{2}=d^{2} & \\
a * g=g * a, & g \in\{b, c, d\} \\
b * g=g * b, & g \in\{c, d\} .
\end{array}
$$

The canonical naming is

$$
\begin{array}{cccc}
e=7 & b=5 & c=3 & d=1 \\
a=6 & x_{1}=4 & x_{2}=2 & x_{3}=0
\end{array}
$$

with all non trivial objects equivalent. The numeric table is 


$\begin{array}{llllllll}7 & 6 & 5 & 4 & 3 & 2 & 1 & 0 \\ 6 & 7 & 4 & 5 & 2 & 3 & 0 & 1 \\ 5 & 4 & 7 & 6 & 1 & 0 & 3 & 2 \\ 4 & 5 & 6 & 7 & 0 & 1 & 2 & 3 \\ 3 & 2 & 1 & 0 & 7 & 6 & 5 & 4 \\ 2 & 3 & 0 & 1 & 6 & 7 & 4 & 5 \\ 1 & 0 & 3 & 2 & 5 & 4 & 7 & 6 \\ 0 & 1 & 2 & 3 & 4 & 5 & 6 & 7\end{array}$

and the canonical representation is

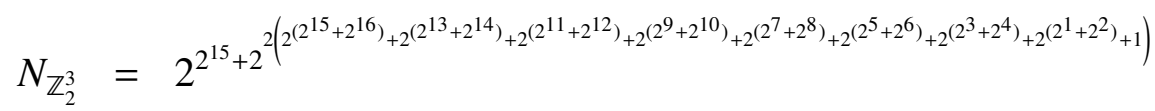

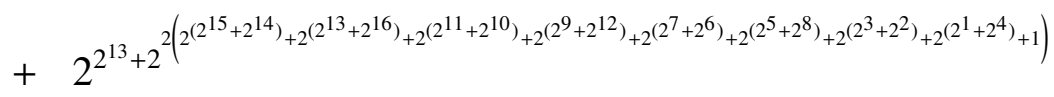

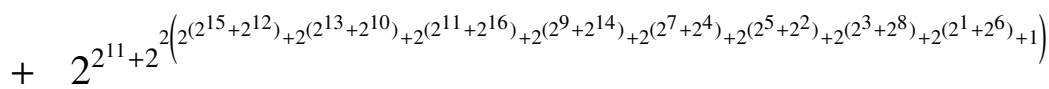

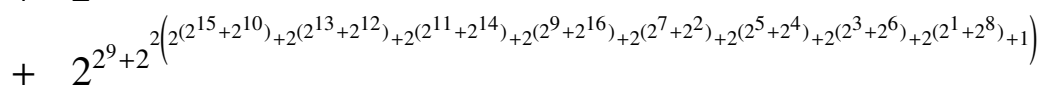

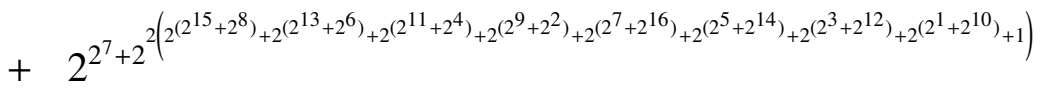

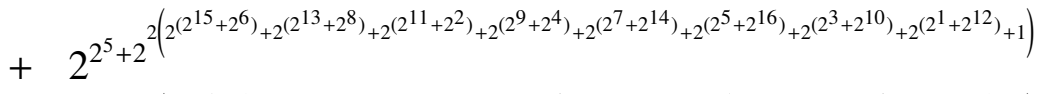

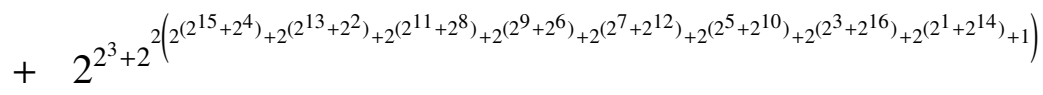

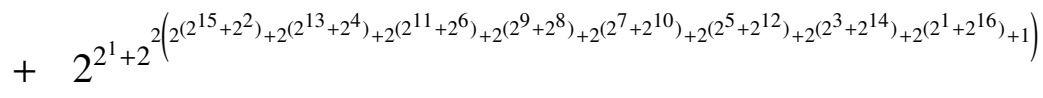

Now we will look for groups with all objects of order 2, and $a * c \neq c * a$. We can take $c * a=d$. This implies $d * a=c$ because $|a|=2$. Since $|d|=2$ we also have $d * c=a$. Also, $a * c=x_{2}$ and $|c|=2$ imply $x_{2} * c=a$. This is a contradiction.

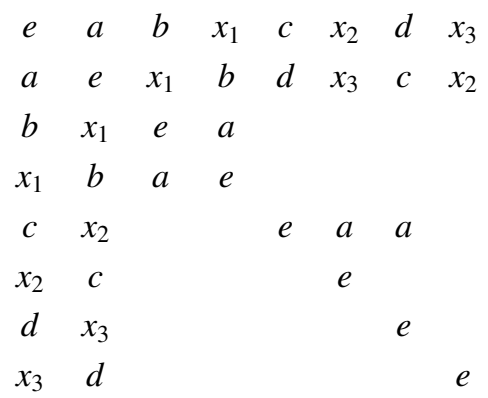

The contradiction does not depend on the first four objects $e, a, b, x_{1}$. This means that any non abelian group of eight objects, must also have objects of order 4 or 8 . In particular, the group $\mathbb{Z}_{2}^{3}$, above, is the only group of eight objects with all non trivial elements of order 2 . 
Dihedral Group $D_{8}$. Now we seek for $G$ with objects of order 4 . We recall we must have a multiple of $2=\phi(4)$, many objects of order 4 . We first consider the case with two objects of order 4 , and five objects of second order. Let $a, b, x_{1}, c, x_{2}$ be the objects of order 2 , and let $d, x_{3}$ our objects of order 4 . We have the form

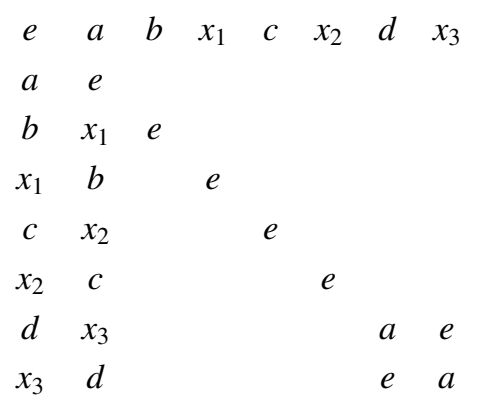

Since $|b|=\left|x_{1}\right|=|c|=\left|x_{2}\right|=2$, we have $x_{1} * b=b * x_{1}=x_{2} * c=c * x_{2}=a$, respectively.

$\begin{array}{cccccccc}e & a & b & x_{1} & c & x_{2} & d & x_{3} \\ a & e & & & & & & \\ b & x_{1} & e & a & & & & \\ x_{1} & b & a & e & & & & \\ c & x_{2} & & & e & a & & \\ x_{2} & c & & & a & e & & \\ d & x_{3} & & & & & a & e \\ x_{3} & d & & & & & e & a\end{array}$

Now, $|b|=\left|x_{1}\right|=|c|=\left|x_{2}\right|=2$ implies $b * a=x_{1}, x_{1} * a=b, c * a=x_{2}, x_{2} * a=c$, respectively. Then, $d * a=x_{3}$ and $x_{3} * a=d$. Notice we are starting to see a block form of the table for $K(4)$. We have $2 \times 2$ blocks forming the Klein group. The blocks of $d, x_{3}$ have not yet interfered with the rest of the blocks. We can suppose $b * c=d$, without loss of generality,

$\begin{array}{llllllll}e & a & b & x_{1} & c & x_{2} & d & x_{3} \\ a & e & x_{1} & b & x_{2} & c & x_{3} & d \\ b & x_{1} & e & a & & & & \\ x_{1} & b & a & e & & & & \\ c & x_{2} & d & x_{3} & e & a & & \\ x_{2} & c & x_{3} & d & a & e & & \\ d & x_{3} & & & & & a & e \\ x_{3} & d & & & & & e & a\end{array}$

The rest of the table is determined. Find $b * d=c$, and $d * c=b$. 


$\begin{array}{cccccccc}e & a & b & x_{1} & c & x_{2} & d & x_{3} \\ a & e & x_{1} & b & x_{2} & c & x_{3} & d \\ b & x_{1} & e & a & & & & \\ x_{1} & b & a & e & & & & \\ c & x_{2} & d & x_{3} & e & a & b & x_{1} \\ x_{2} & c & x_{3} & d & a & e & x_{1} & b \\ d & x_{3} & c & x_{2} & & & a & e \\ x_{3} & d & x_{2} & c & & & e & a\end{array}$

Now we can use $c=b * d$ to find $c * d=b *(d * d)=b * a=x_{1}$.

$\begin{array}{cccccccc}e & a & b & x_{1} & c & x_{2} & d & x_{3} \\ a & e & x_{1} & b & x_{2} & c & x_{3} & d \\ b & x_{1} & e & a & & & & \\ x_{1} & b & a & e & & & & \\ c & x_{2} & d & x_{3} & e & a & b & x_{1} \\ x_{2} & c & x_{3} & d & a & e & x_{1} & b \\ d & x_{3} & c & x_{2} & x_{1} & b & a & e \\ x_{3} & d & x_{2} & c & b & x_{1} & e & a\end{array}$

The rest of the table is determined similarly. For example, $|c|=2$ implies $c * b=x_{3}$.

$\begin{array}{cccccccc}e & a & b & x_{1} & c & x_{2} & d & x_{3} \\ a & e & x_{1} & b & x_{2} & c & x_{3} & d \\ b & x_{1} & e & a & x_{3} & d & c & x_{2} \\ x_{1} & b & a & e & d & x_{3} & x_{2} & c \\ c & x_{2} & d & x_{3} & e & a & b & x_{1} \\ x_{2} & c & x_{3} & d & a & e & x_{1} & b \\ d & x_{3} & c & x_{2} & x_{1} & b & a & e \\ x_{3} & d & x_{2} & c & b & x_{1} & e & a\end{array}$

This is the Dihedral group, $D_{8}$, defined by $|G|=8$ and the set of equations

$$
\begin{aligned}
& e=a^{2}=b^{2}=x_{1}^{2}=c^{2}=x_{2}^{2} \\
& (b * c)^{2}=a
\end{aligned}
$$

It is the only group $|G|=8$, with exactly two objects of order 4 , and five objects of order 2 . Notice that the set of equations only mentions seven different objects. The eighth object is $a *(b * c)$, and it is also of order 4 , just as $b * c$. Now we will find the canonical naming function of this group. We write the group with generic symbols, 


$\begin{array}{cccccccc}e & g_{1} & g_{2} & g_{3} & g_{4} & g_{5} & g_{6} & g_{7} \\ g_{1} & e & g_{3} & g_{2} & g_{5} & g_{4} & g_{7} & g_{6} \\ g_{2} & g_{3} & g_{1} & e & g_{7} & g_{6} & g_{4} & g_{5} \\ g_{3} & g_{2} & e & g_{1} & g_{6} & g_{7} & g_{5} & g_{4} \\ g_{4} & g_{5} & g_{6} & g_{7} & e & g_{1} & g_{2} & g_{3} \\ g_{5} & g_{4} & g_{7} & g_{6} & g_{1} & e & g_{3} & g_{2} \\ g_{6} & g_{7} & g_{5} & g_{4} & g_{3} & g_{2} & e & g_{1} \\ g_{7} & g_{6} & g_{4} & g_{5} & g_{2} & g_{3} & g_{1} & e\end{array}$

and now we will use the letters $a, b, \ldots, x_{1}, x_{2}, \ldots$ as auxiliary variables to find the canonical naming. We want to avoid confusion with the fact that we just used the same symbols $a, b, \ldots, x_{1}, x_{2}, \ldots$ as auxiliary variables to find the group. If we wish to maximize our representation, the first thing we will have to do is find two objects of order 2 that commute. We start with $e=7$, and an arbitrary object, $a=6$, of order 2 . We add an object $b=5$, and if we want to maximize we have to make $x_{1}=a * b=4$.

$\begin{array}{cccc}e & a & b & x_{1} \\ a & e & & \\ b & x_{1} & & \\ x_{1} & b & & \end{array}$

Now we we will choose $b$ that commutes with $a$. There are many options. We simply need two objects $a, b$ that satisfy $e=a^{2}$ and $a * b=b * a$. That gives us the partial table,

$\begin{array}{cccc}e & a & b & x_{1} \\ a & e & x_{1} & b \\ b & x_{1} & & \\ x_{1} & b & & \end{array}$

If we choose, $a=g_{1}$, we have at least one choice of $b$ and we can find $b$ such that $|b|=2$, maximizing the representation.

$\begin{array}{cccc}e & a & b & x_{1} \\ a & e & x_{1} & b \\ b & x_{1} & e & a \\ x_{1} & b & a & e\end{array}$

We simply need three objects $a, b, x_{1}$, of order 2 , such that $a$ commutes with $b, x_{1}$. We have several options to do this. For example, $g_{1}, g_{4}, g_{5}, g_{6}, g_{7}$ are all of order 2 and each object in this list commutes with two others of the list. Therefore, any of these objects can take the place of $a$, for now. Add another object to the table, say $c=3$, and consequently $x_{2}=a * c=2$. Then, we need to add another object $d=1$, for the product $b * c=d$. Finally we have $x_{3}=a * d=0$. 


$\begin{array}{llllllll}e & a & b & x_{1} & c & x_{2} & d & x_{3} \\ a & e & x_{1} & b & & & & \\ b & x_{1} & e & a & & & & \\ x_{1} & b & a & e & & & & \\ c & x_{2} & d & x_{3} & & & & \\ x_{2} & c & x_{3} & d & & & & \\ d & x_{3} & c & x_{2} & & & & \\ x_{3} & d & x_{2} & c & & & & \end{array}$

The table of this group tells us it is possible to find $c$ that commutes with $a$. This implies $a$ also commutes with $a * c$. The only object that commutes with four objects, of order 2 , is $g_{1}$. We have reduced to eight possible combinations, the canonical naming function. Our candidates are

$\begin{array}{cccccccc}e=7 & e=7 & e=7 & e=7 & e=7 & e=7 & e=7 & e=7 \\ g_{1}=a=6 & g_{1}=a=6 & g_{1}=a=6 & g_{1}=a=6 & g_{1}=a=6 & g_{1}=a=6 & g_{1}=a=6 & g_{1}=a=6 \\ g_{4}=b=5 & g_{4}=b=5 & g_{5}=b=5 & g_{5}=b=5 & g_{6}=b=5 & g_{6}=b=5 & g_{7}=b=5 & g_{7}=b=5 \\ g_{5}=x_{1}=4 & g_{5}=x_{1}=4 & g_{4}=x_{1}=4 & g_{4}=x_{1}=4 & g_{7}=x_{1}=4 & g_{7}=x_{1}=4 & g_{6}=x_{1}=4 & g_{6}=x_{1}=4 \\ g_{6}=c=3 & g_{7}=c=3 & g_{6}=c=3 & g_{7}=c=3 & g_{4}=c=3 & g_{5}=c=3 & g_{4}=c=3 & g_{5}=c=3 \\ g_{7}=x_{2}=2 & g_{6}=x_{2}=2 & g_{7}=x_{2}=2 & g_{6}=x_{2}=2 & g_{5}=x_{2}=2 & g_{4}=x_{2}=2 & g_{5}=x_{2}=2 & g_{4}=x_{2}=2\end{array}$

Any of these naming functions gives the table

$\begin{array}{cccccccc}e & a & b & x_{1} & c & x_{2} & d & x_{3} \\ a & e & x_{1} & b & x_{2} & c & x_{3} & d \\ b & x_{1} & e & a & & & & \\ x_{1} & b & a & e & & & & \\ c & x_{2} & d & x_{3} & & & & \\ x_{2} & c & x_{3} & d & & & & \\ d & x_{3} & & & & & & \\ x_{3} & d & & & & & & \end{array}$

We see none of our choices of naming functions will have $b * c=c * b$. For example, in the first naming function, $g_{4}, g_{6}$ do not commute. In the second naming function $g_{4}, g_{7}$ do not commute, etc.

$\begin{array}{cccccccc}e & a & b & x_{1} & c & x_{2} & d & x_{3} \\ a & e & x_{1} & b & x_{2} & c & x_{3} & d \\ b & x_{1} & e & a & x_{3} & d & & \\ x_{1} & b & a & e & d & x_{3} & & \\ c & x_{2} & d & x_{3} & & & & \\ x_{2} & c & x_{3} & d & & & & \\ d & x_{3} & & & & & & \\ x_{3} & d & & & & & & \end{array}$

Notice that in the eight possible naming functions, we have $c^{2}=e$. 


$\begin{array}{cccccccc}e & a & b & x_{1} & c & x_{2} & d & x_{3} \\ a & e & x_{1} & b & x_{2} & c & x_{3} & d \\ b & x_{1} & e & a & x_{3} & d & & \\ x_{1} & b & a & e & d & x_{3} & & \\ c & x_{2} & d & x_{3} & e & a & & \\ x_{2} & c & x_{3} & d & a & e & & \\ d & x_{3} & & & & & & \\ x_{3} & d & & & & & & \end{array}$

The rest of the table is determined.

$\begin{array}{cccccccc}e & a & b & x_{1} & c & x_{2} & d & x_{3} \\ a & e & x_{1} & b & x_{2} & c & x_{3} & d \\ b & x_{1} & e & a & x_{3} & d & x_{2} & c \\ x_{1} & b & a & e & d & x_{3} & c & x_{2} \\ c & x_{2} & d & x_{3} & e & a & b & x_{1} \\ x_{2} & c & x_{3} & d & a & e & x_{1} & b \\ d & x_{3} & c & x_{2} & x_{1} & b & a & e \\ x_{3} & d & x_{2} & c & b & x_{1} & e & a\end{array}$

This means the order 4 objects, $g_{2}, g_{3}$, are equivalent and if one is assigned $d=1$, the other is $x_{3}=0$. Also, $g_{4}, g_{5}$ are equivalent, and $g_{6}, g_{7}$ are equivalent. A canonical naming function would be

$$
\begin{array}{ll}
e=7 & g_{6}=3 \\
g_{1}=6 & g_{7}=2 \\
g_{4}=5 & g_{2}=1 \\
g_{5}=4 & g_{3}=0
\end{array}
$$

The numeric table is

$\begin{array}{llllllll}7 & 6 & 5 & 4 & 3 & 2 & 1 & 0 \\ 6 & 7 & 4 & 5 & 2 & 3 & 0 & 1 \\ 5 & 4 & 7 & 6 & 0 & 1 & 2 & 3 \\ 4 & 5 & 6 & 7 & 1 & 0 & 3 & 2 \\ 3 & 2 & 1 & 0 & 7 & 6 & 5 & 4 \\ 2 & 3 & 0 & 1 & 6 & 7 & 4 & 5 \\ 1 & 0 & 3 & 2 & 4 & 5 & 6 & 7 \\ 0 & 1 & 2 & 3 & 5 & 4 & 7 & 6\end{array}$

and the canonical representation is 


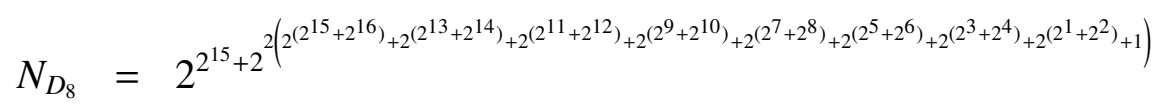

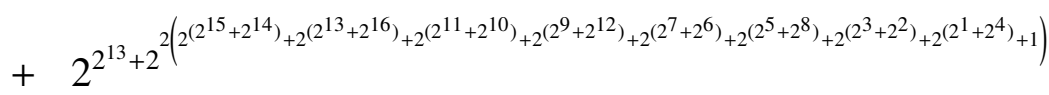

$$
\begin{aligned}
& +2^{\left.2^{11}+2^{2\left(2^{\left(2^{15}\right.}+2^{12}\right)}+2^{\left(2^{13}+2^{10}\right)}+2^{\left(2^{11}+2^{16}\right)}+2^{\left(2^{9}+2^{14}\right)}+2^{\left(2^{7}+2^{4}\right)}+2^{\left(2^{5}+2^{2}\right)}+2^{\left(2^{3}+2^{8}\right)}+2^{\left(2^{1}+2^{6}\right)}+1\right)}
\end{aligned}
$$

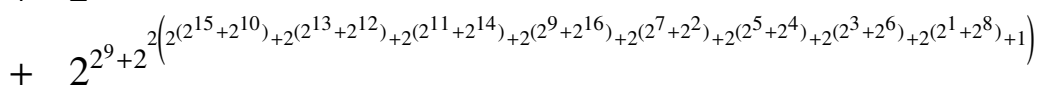

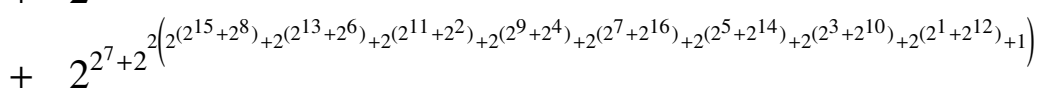

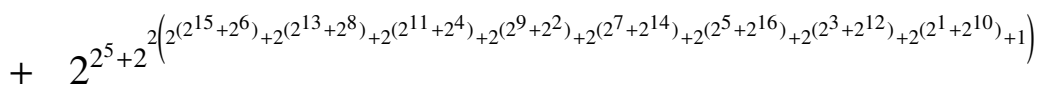

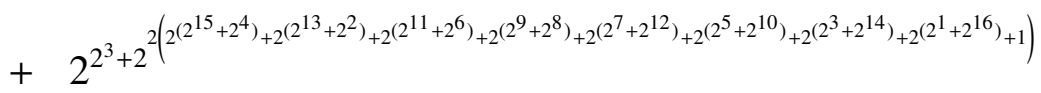

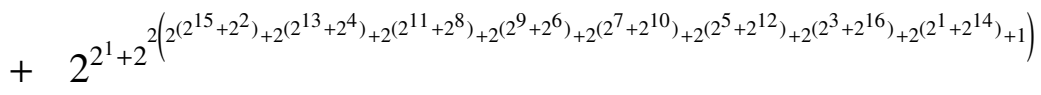

Direct Product $\mathbb{Z}_{2} \oplus \mathbb{Z}_{4}$. Now we will consider groups with four objects of order 4 , and three objects of order 2 . Let $a, b$ be two objects of order 2 . Then $|a * b|=2$, also. Let $x_{1}=a * b$, and $c$ an object of order 4 , then we have

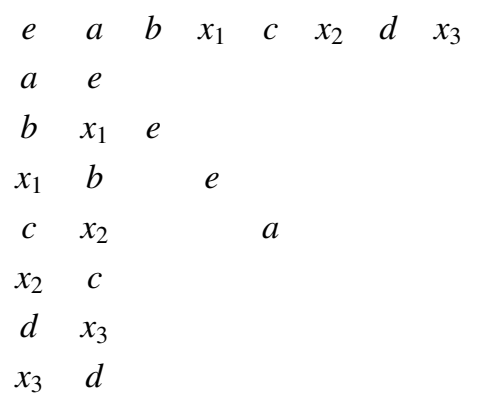

We use $|b|=\left|x_{1}\right|=2$ to find $x_{1} * b=b * x_{1}=a$, respectively.

$\begin{array}{cccccccc}e & a & b & x_{1} & c & x_{2} & d & x_{3} \\ a & e & & & & & & \\ b & x_{1} & e & a & & & & \\ x_{1} & b & a & e & & & & \\ c & x_{2} & & & a & & & \\ x_{2} & c & & & & & \\ d & x_{3} & & & & & & \\ x_{3} & d & & & & & & \end{array}$

Now we use $|b|=\left|x_{1}\right|=2$ to find $b * a=x_{1}$ and $x_{1} * a=b$, respectively. 


$\begin{array}{llllllll}e & a & b & x_{1} & c & x_{2} & d & x_{3} \\ a & e & x_{1} & b & & & & \\ b & x_{1} & e & a & & & & \\ x_{1} & b & a & e & & & \\ c & x_{2} & & & a & & \\ x_{2} & c & & & & & \\ d & x_{3} & & & & & \\ x_{3} & d & & & & & & \end{array}$

We have the arrows $e \rightarrow c, c \rightarrow a, a \rightarrow x_{2}$, and finally the incomplete arrow $x_{2} \rightarrow$, going from the first row to the fifth row. Given that $|c|=4$, must have $x_{2} \rightarrow e$.

$\begin{array}{cccccccc}e & a & b & x_{1} & c & x_{2} & d & x_{3} \\ a & e & x_{1} & b & & & & \\ b & x_{1} & e & a & & & & \\ x_{1} & b & a & e & & & & \\ c & x_{2} & & & a & e & & \\ x_{2} & c & & & e & a & & \\ d & x_{3} & & & & & & \\ x_{3} & d & & & & & & \end{array}$

We suppose, without loss of generality, $b * c=d$. Then, we use $|b|=2$ to find $b * d=c$.

$\begin{array}{cccccccc}e & a & b & x_{1} & c & x_{2} & d & x_{3} \\ a & e & x_{1} & b & & & & \\ b & x_{1} & e & a & & & & \\ x_{1} & b & a & e & & & & \\ c & x_{2} & d & x_{3} & a & e & & \\ x_{2} & c & x_{3} & d & e & a & & \\ d & x_{3} & c & x_{2} & & & & \\ x_{3} & d & x_{2} & c & & & & \end{array}$

We look at the arrows of the first and fifth row, again. But now, we focus on the other set of arrows

$$
\begin{aligned}
& b \rightarrow d \\
& d \rightarrow \\
& x_{1} \rightarrow x_{3} \\
& x_{3} \rightarrow
\end{aligned}
$$

Since $|c|=4$, we have $d * c=x_{1}$ and $x_{3} * c=b$. We can repeat the same process with the first and sixth row. This is the same thing we have been doing with objects of second order, but now with objects of fourth order. 


$\begin{array}{cccccccc}e & a & b & x_{1} & c & x_{2} & d & x_{3} \\ a & e & x_{1} & b & x_{2} & c & x_{3} & d \\ b & x_{1} & e & a & & & & \\ x_{1} & b & a & e & & & & \\ c & x_{2} & d & x_{3} & a & e & x_{1} & b \\ x_{2} & c & x_{3} & d & e & a & b & x_{1} \\ d & x_{3} & c & x_{2} & & & & \\ x_{3} & d & x_{2} & c & & & & \end{array}$

Now we use the fact $|d|=4$,

$\begin{array}{cccccccc}e & a & b & x_{1} & c & x_{2} & d & x_{3} \\ a & e & x_{1} & b & x_{2} & c & x_{3} & d \\ b & x_{1} & e & a & & & & \\ x_{1} & b & a & e & & & & \\ c & x_{2} & d & x_{3} & a & e & x_{1} & b \\ x_{2} & c & x_{3} & d & e & a & b & x_{1} \\ d & x_{3} & c & x_{2} & & & a & e \\ x_{3} & d & x_{2} & c & & & e & a\end{array}$

We observe $|d|=4$ to find $d * b=c$ and $d * x_{1}=x_{2}$. We do the same with the last column.

$\begin{array}{cccccccc}e & a & b & x_{1} & c & x_{2} & d & x_{3} \\ a & e & x_{1} & b & x_{2} & c & x_{3} & d \\ b & x_{1} & e & a & & & c & x_{2} \\ x_{1} & b & a & e & & & x_{2} & c \\ c & x_{2} & d & x_{3} & a & e & x_{1} & b \\ x_{2} & c & x_{3} & d & e & a & b & x_{1} \\ d & x_{3} & c & x_{2} & & & a & e \\ x_{3} & d & x_{2} & c & & & e & a\end{array}$

We use $c=d * b$ to find $c * b=d *(b * b)=d$. Use $c=b * d$ to find $c * d=b *(d * d)=b * a=x_{1}$.

$\begin{array}{cccccccc}e & a & b & x_{1} & c & x_{2} & d & x_{3} \\ a & e & x_{1} & b & x_{2} & c & x_{3} & d \\ b & x_{1} & e & a & d & x_{3} & c & x_{2} \\ x_{1} & b & a & e & x_{3} & d & x_{2} & c \\ c & x_{2} & d & x_{3} & a & e & x_{1} & b \\ x_{2} & c & x_{3} & d & e & a & b & x_{1} \\ d & x_{3} & c & x_{2} & x_{1} & b & a & e \\ x_{3} & d & x_{2} & c & b & x_{1} & e & a\end{array}$

This is the direct product group $\mathbb{Z}_{2} \oplus \mathbb{Z}_{4}$. To define our group, we had to set three second order objects, and one object, $c$, of fourth order. Then we defined $d=b * c$, and we used $d^{2}=a$. This group is defined by the set of equations 


$$
\begin{aligned}
& e=a^{2}=b^{2}=x_{1}^{2} \\
& a=c^{2}=(b * c)^{2}
\end{aligned}
$$

To find the canonical naming, we write the table in terms of $g_{i}$.

$\begin{array}{cccccccc}e & g_{1} & g_{2} & g_{3} & g_{4} & g_{5} & g_{6} & g_{7} \\ g_{1} & e & g_{3} & g_{2} & g_{5} & g_{4} & g_{7} & g_{6} \\ g_{2} & g_{3} & e & g_{1} & g_{6} & g_{7} & g_{4} & g_{5} \\ g_{3} & g_{2} & g_{1} & e & g_{7} & g_{6} & g_{5} & g_{4} \\ g_{4} & g_{5} & g_{6} & g_{7} & g_{1} & e & g_{3} & g_{2} \\ g_{5} & g_{4} & g_{7} & g_{6} & e & g_{1} & g_{2} & g_{3} \\ g_{6} & g_{7} & g_{4} & g_{5} & g_{3} & g_{2} & g_{1} & e \\ g_{7} & g_{6} & g_{5} & g_{4} & g_{2} & g_{3} & e & g_{1}\end{array}$

To find the canonical naming, we begin with $e=7$. By experience, we already know we will have to name $g_{1}=a=6$. Then, we will have to find two second order objects, which in this case are $g_{2}, g_{3}$. We get the table

$\begin{array}{cccc}e & a & b & x_{1} \\ a & e & x_{1} & b \\ b & x_{1} & e & a \\ x_{1} & b & a & e\end{array}$

We add an object $c$, and then we have to add $x_{2}, d, x_{3}$. But this already determines our group. The objects of fourth order are equivalent because we can choose arbitrary $c$ with order 4 , and the rest of the naming values are defined. Therefore, we have a canonical naming with $g_{2}=b=5, g_{3}=x_{1}=4, g_{4}=c=3, a * c=g_{5}=x_{2}=2, g_{7}=d=1, a * d=g_{6}=0$. In this case, we built our group in the order of the canonical naming. The numeric table is

$\begin{array}{llllllll}7 & 6 & 5 & 4 & 3 & 2 & 1 & 0 \\ 6 & 7 & 4 & 5 & 2 & 3 & 0 & 1 \\ 5 & 4 & 7 & 6 & 1 & 0 & 3 & 2 \\ 4 & 5 & 6 & 7 & 0 & 1 & 2 & 3 \\ 3 & 2 & 1 & 0 & 6 & 7 & 4 & 5 \\ 2 & 3 & 0 & 1 & 7 & 6 & 5 & 4 \\ 1 & 0 & 3 & 2 & 4 & 5 & 6 & 7 \\ 0 & 1 & 2 & 3 & 5 & 4 & 7 & 6\end{array}$

The canonical representation is 


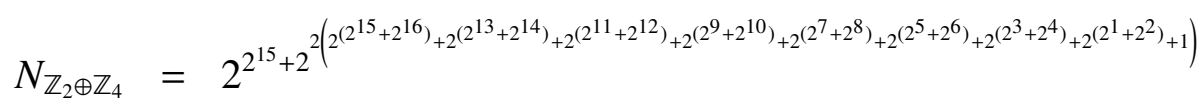

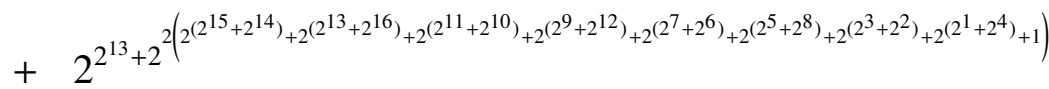

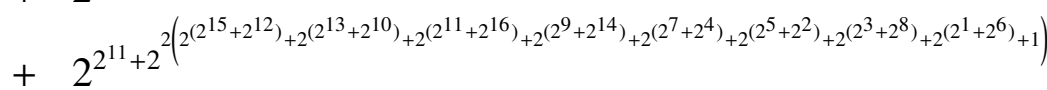

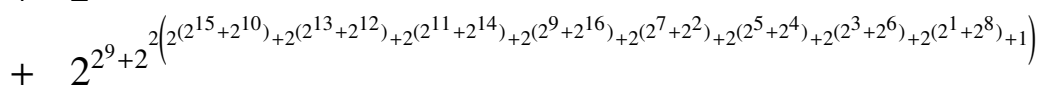

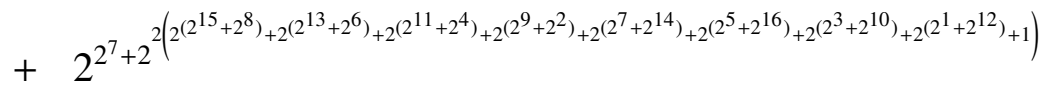

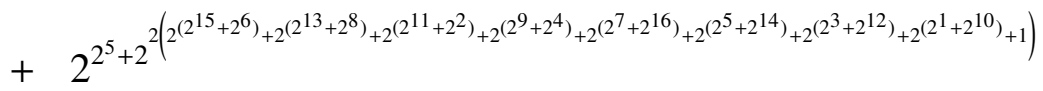

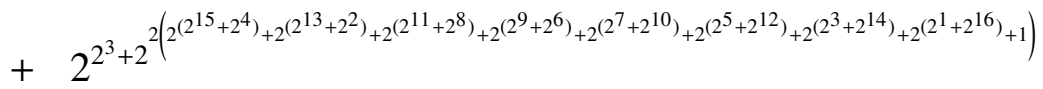

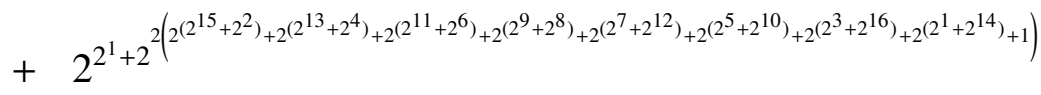

Quaternion Group $Q_{8}$. Consider $G$ with six objects of order 4. Let $a$ be our only object of second order.

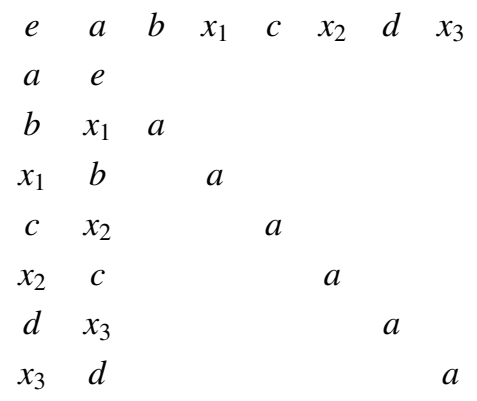

Now we need to make $x_{1} * b=e$ because $|b|=4$.

$\begin{array}{cccccccc}e & a & b & x_{1} & c & x_{2} & d & x_{3} \\ a & e & & & & & & \\ b & x_{1} & a & e & & & & \\ x_{1} & b & e & a & & & & \\ c & x_{2} & & & a & & & \\ x_{2} & c & & & & a & & \\ d & x_{3} & & & & & a & \\ x_{3} & d & & & & & & a\end{array}$

Again, we use $|b|=4$ to find $b * a=x_{1}$. 


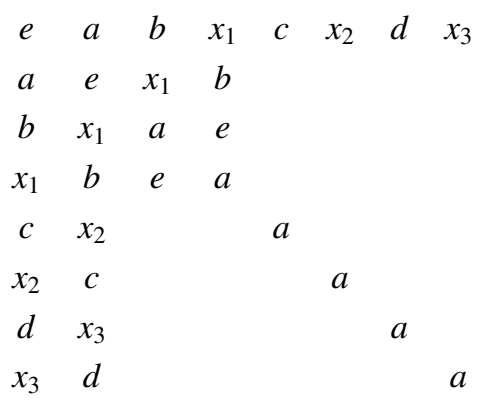

We have, without loss of generality, $b * c=d$,

$\begin{array}{cccccccc}e & a & b & x_{1} & c & x_{2} & d & x_{3} \\ a & e & x_{1} & b & & & & \\ b & x_{1} & a & e & & & & \\ x_{1} & b & e & a & & & & \\ c & x_{2} & d & x_{3} & a & & & \\ x_{2} & c & x_{3} & d & & a & & \\ d & x_{3} & x_{2} & c & & & a & \\ x_{3} & d & c & x_{2} & & & & a\end{array}$

We use $|c=| 4$ to find $x_{2} * c=e$.

$\begin{array}{cccccccc}e & a & b & x_{1} & c & x_{2} & d & x_{3} \\ a & e & x_{1} & b & & & & \\ b & x_{1} & a & e & & & & \\ x_{1} & b & e & a & & & & \\ c & x_{2} & d & x_{3} & a & e & & \\ x_{2} & c & x_{3} & d & e & a & & \\ d & x_{3} & x_{2} & c & & & a & e \\ x_{3} & d & c & x_{2} & & & e & a\end{array}$

Again we use $|c|=4$ to find $c * a=x_{2}$. In the same way we use $|d|=4$ to find $d * a=x_{3}$.

$\begin{array}{cccccccc}e & a & b & x_{1} & c & x_{2} & d & x_{3} \\ a & e & x_{1} & b & x_{2} & c & x_{3} & d \\ b & x_{1} & a & e & & & & \\ x_{1} & b & e & a & & & & \\ c & x_{2} & d & x_{3} & a & e & & \\ x_{2} & c & x_{3} & d & e & a & & \\ d & x_{3} & x_{2} & c & & & a & e \\ x_{3} & d & c & x_{2} & & & e & a\end{array}$

Now we can use $c=x_{1} * d$ to fins $c * d=x_{1} *(d * d)=x_{1} * a=b$. 


$\begin{array}{llllllll}e & a & b & x_{1} & c & x_{2} & d & x_{3} \\ a & e & x_{1} & b & x_{2} & c & x_{3} & d \\ b & x_{1} & a & e & & & & \\ x_{1} & b & e & a & & & & \\ c & x_{2} & d & x_{3} & a & e & & \\ x_{2} & c & x_{3} & d & e & a & & \\ d & x_{3} & x_{2} & c & b & x_{1} & a & e \\ x_{3} & d & c & x_{2} & x_{1} & b & e & a\end{array}$

The rest of the table is determined as usual.

$\begin{array}{cccccccc}e & a & b & x_{1} & c & x_{2} & d & x_{3} \\ a & e & x_{1} & b & x_{2} & c & x_{3} & d \\ b & x_{1} & a & e & x_{3} & d & c & x_{2} \\ x_{1} & b & e & a & d & x_{3} & x_{2} & c \\ c & x_{2} & d & x_{3} & a & e & x_{1} & b \\ x_{2} & c & x_{3} & d & e & a & b & x_{1} \\ d & x_{3} & x_{2} & c & b & x_{1} & a & e \\ x_{3} & d & c & x_{2} & x_{1} & b & e & a\end{array}$

This group was determined by the conditions of having one second order object, $a$, and $a=b^{2}=x_{1}^{2}=c^{2}=x_{2}^{2}=d^{2}$, where $d=b * c$. Thus, the system of equations

$$
\begin{aligned}
& e=a^{2} \\
& a=b^{2}=(a * b)^{2}=c^{2}=(a * c)^{2}=(b * c)^{2}
\end{aligned}
$$

determines the group of quaternions, $Q_{8}$. Notice we did not include $a=(c * b)^{2}$, because it is implied by the others. To find the canonical naming function, we start with $e=7$ and $a=6$ and a fourth order object $b=5$. Then, we have to choose $c=3$, then $d=1$, and we can make these choices arbitrarily. We have a canonical naming $e=7, a=6, b=5, x_{1}=4, c=3, x_{2}=2$, $d=1, x_{3}=0$, and all the fourth order objects are equivalent.

$\begin{array}{llllllll}7 & 6 & 5 & 4 & 3 & 2 & 1 & 0 \\ 6 & 7 & 4 & 5 & 2 & 3 & 0 & 1 \\ 5 & 4 & 6 & 7 & 0 & 1 & 3 & 2 \\ 4 & 5 & 7 & 6 & 1 & 0 & 2 & 3 \\ 3 & 2 & 1 & 0 & 6 & 7 & 4 & 5 \\ 2 & 3 & 0 & 1 & 7 & 6 & 5 & 4 \\ 1 & 0 & 2 & 3 & 5 & 4 & 6 & 7 \\ 0 & 1 & 3 & 2 & 4 & 5 & 7 & 6\end{array}$

The canonical representation being 


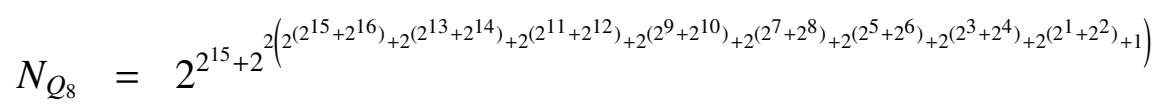

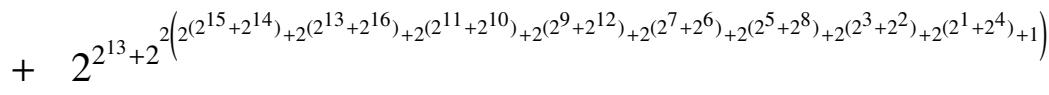

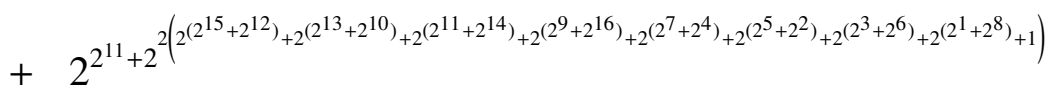

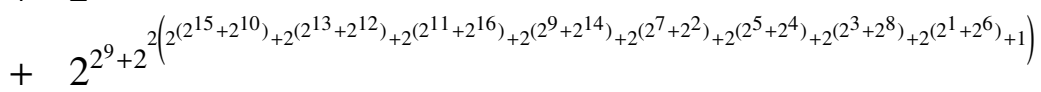

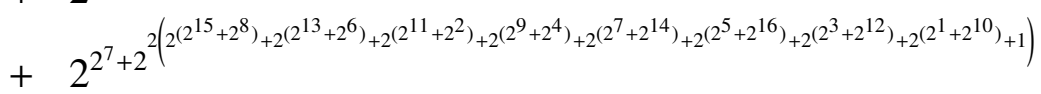

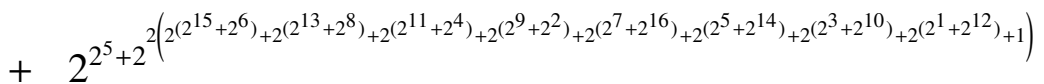

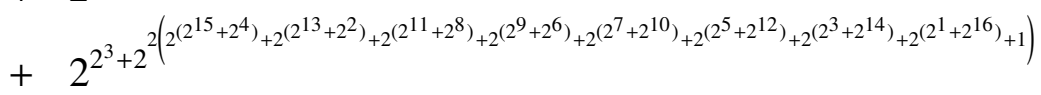

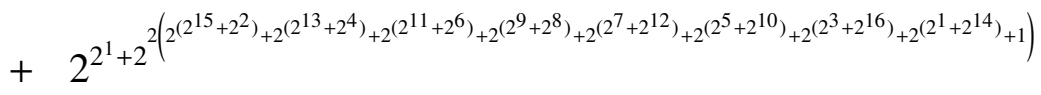

Cyclic Group $\mathbb{Z}_{8}$. Finding the cyclic group is trivial, and it is defined by $|G|=8$ and the equations $e=a^{2}, a=b^{2}, c=b^{2}$. It has numeric table

$\begin{array}{llllllll}7 & 6 & 5 & 4 & 3 & 2 & 1 & 0 \\ 6 & 5 & 4 & 3 & 2 & 1 & 0 & 7 \\ 5 & 4 & 3 & 2 & 1 & 0 & 7 & 6 \\ 4 & 3 & 2 & 1 & 0 & 7 & 6 & 5 \\ 3 & 2 & 1 & 0 & 7 & 6 & 5 & 4 \\ 2 & 1 & 0 & 7 & 6 & 5 & 4 & 3 \\ 1 & 0 & 7 & 6 & 5 & 4 & 3 & 2 \\ 0 & 7 & 6 & 5 & 4 & 3 & 2 & 1\end{array}$

The canonical representation of this group being

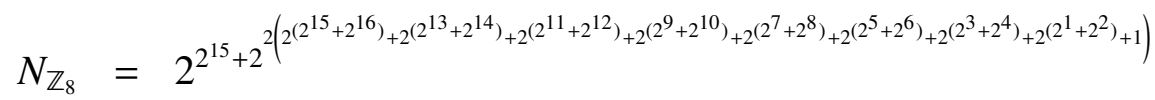

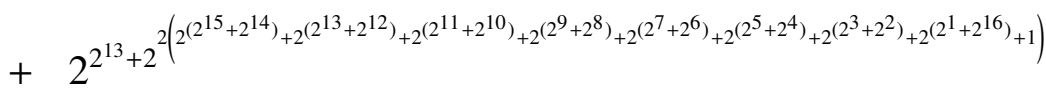

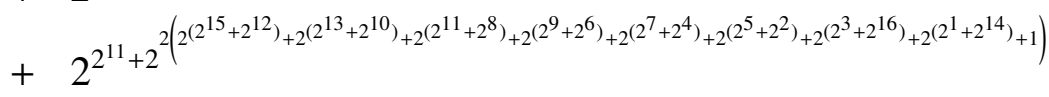

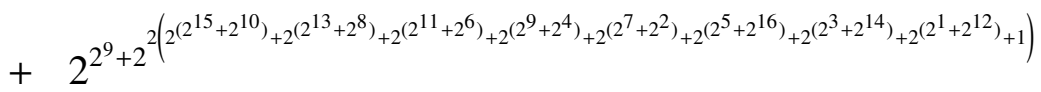

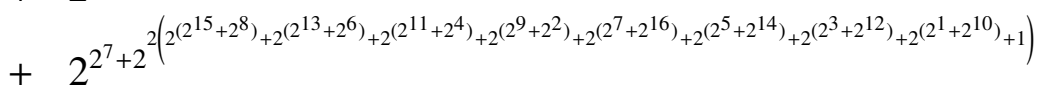

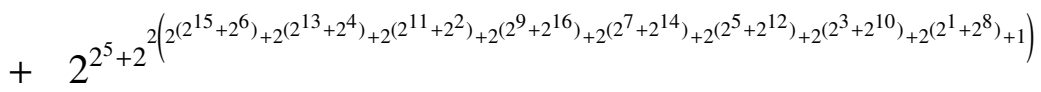

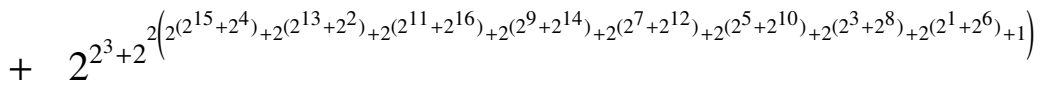

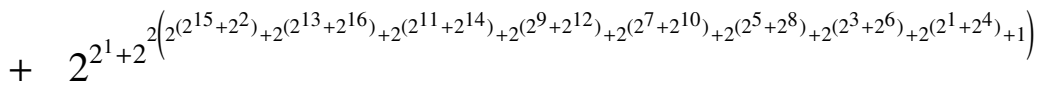

We have ordered groups of eight objects. The order is $\mathbb{Z}_{8}<Q_{8}<D_{8}<\mathbb{Z}_{2} \oplus \mathbb{Z}_{4}<\mathbb{Z}_{2}^{3}$. 


\section{6 $|G|=9$}

We now find the groups of nine objects. There are two different isomorphism classes. We know that if $|G|=9$ then $|g|=3$ or $|g|=9$ for any $g \in G$.

Direct Product $\mathbb{Z}_{3} \oplus \mathbb{Z}_{3}$. We start looking for groups with all objects of order 3. We start with the list of objects.

$$
\begin{array}{lllllllll}
e & a & x_{1} & b & x_{2} & x_{3} & c & x_{4} & x_{5} \\
a & x_{1} & & & & & & & \\
x_{1} & e & & & & & & \\
b & x_{2} & & & & & \\
x_{2} & x_{3} & & & & & \\
x_{3} & b & & & & & \\
c & x_{4} & & & & \\
x_{4} & x_{5} & & & & \\
x_{5} & c & & & & & & &
\end{array}
$$

We know the function $* x_{1}$ is equal to the composition $* a \circ * a$ so that

$$
\begin{array}{ccccccccc}
e & a & x_{1} & b & x_{2} & x_{3} & c & x_{4} & x_{5} \\
a & x_{1} & e & & & & & & \\
x_{1} & e & a & & & & & & \\
b & x_{2} & x_{3} & & & & & \\
x_{2} & x_{3} & b & & & & & \\
x_{3} & b & x_{2} & & & & & \\
c & x_{4} & x_{5} & & & & & \\
x_{4} & x_{5} & c & & & & & \\
x_{5} & c & x_{4} & & & & & &
\end{array}
$$

Since $b$ is also of order 3 , then we have

$$
\begin{array}{ccccccccc}
e & a & x_{1} & b & x_{2} & x_{3} & c & x_{4} & x_{5} \\
a & x_{1} & e & & & & & & \\
x_{1} & e & a & & & & & & \\
b & x_{2} & x_{3} & c & & & & \\
x_{2} & x_{3} & b & & & & & \\
x_{3} & b & x_{2} & & & & & \\
c & x_{4} & x_{5} & e & & & & \\
x_{4} & x_{5} & c & & & & & \\
x_{5} & c & x_{4} & & & & & &
\end{array}
$$




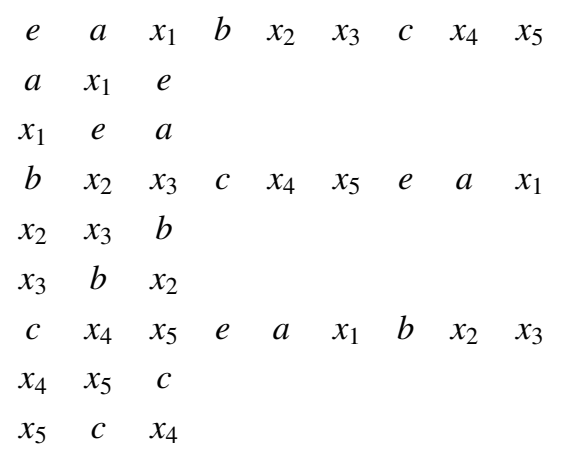

Let us see if we can have a non abelian group. The first option for this is $b * a=x_{3}$, which implies $x_{2} * a=b$.

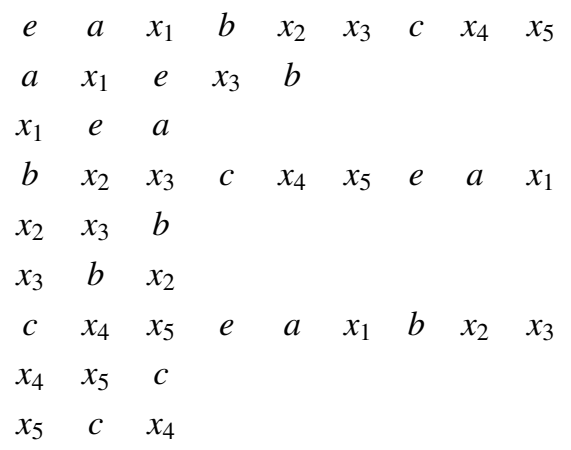

We have a contradiction because $\left|x_{2}\right|=3$ implies $x_{2} * x_{4}=a$. Let us verify we also get a contradiction if $b * a=x_{4}$.

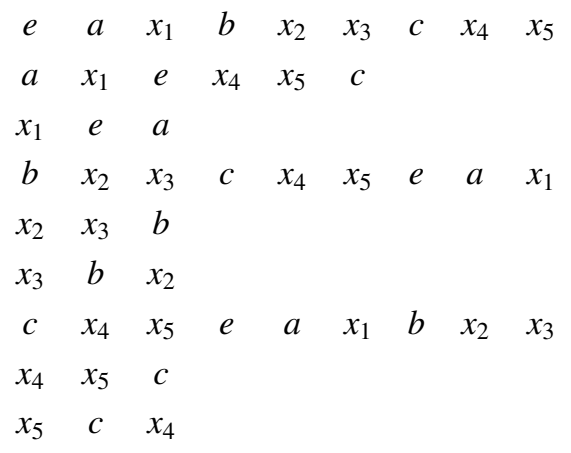

This table leads to contradiction because $\left|x_{3}\right|=3$ implies $x_{3} * x_{1}=a$. The supposition $b * a=x_{5}$ leads to contradiction also.

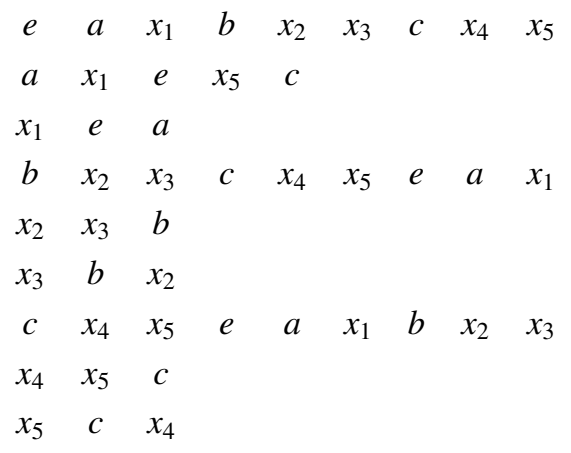


The table shows $x_{2} * a=c$ and $x_{2} * c=a$, which is a contradiction with the fact that $\left|x_{2}\right|=3$. We conclude $b * a=x_{2}$. From this we can trivially find $x_{2} * a=x_{3}$ and $x_{3} * a=b$. We use this last expression to find $b * x_{1}=x_{3}$.

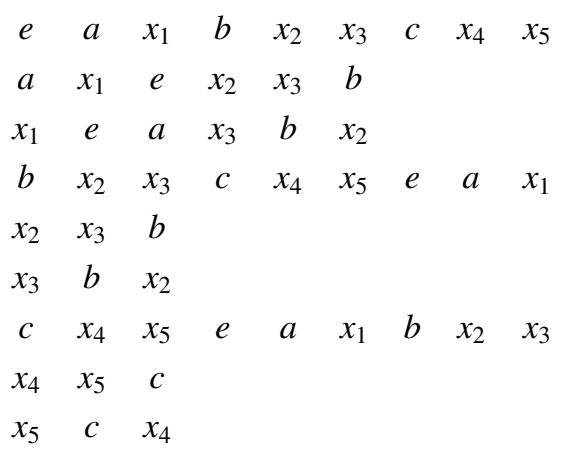

Use the expression $b=x_{2} * x_{1}$ to find $b * x_{2}=x_{4}$.

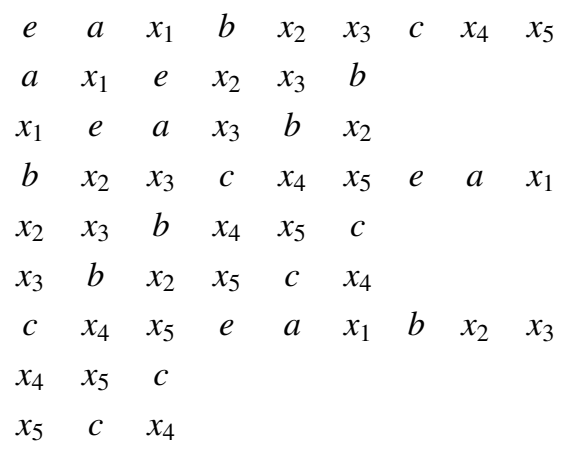

Now use $b=x_{2} * x_{1}$ to find $b * x_{4}=a$.

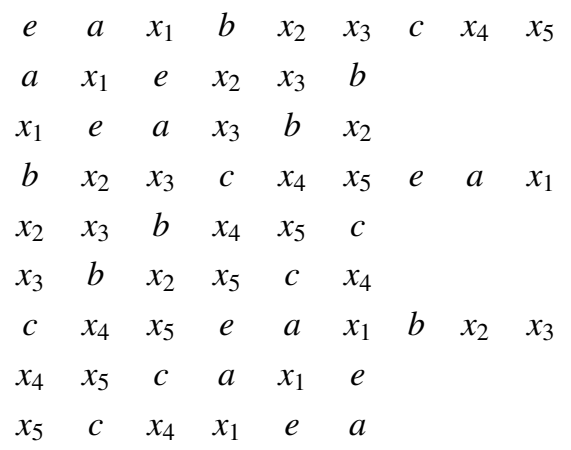

Finding the rest of the table is trivial. We have the direct product group $\mathbb{Z}_{3} \oplus \mathbb{Z}_{3}$. The system of equations that defines this group is given by the relations

$$
\begin{aligned}
x_{1} & =a^{2} \\
c & =b^{2} \\
e & =a^{3}=b^{3} \\
a * b & =b * a
\end{aligned}
$$


Now let us find the canonical naming function of this group. We begin by expressing the table in generic variables $g_{i}$.

$\begin{array}{lcccccccc}e & g_{1} & g_{2} & g_{3} & g_{4} & g_{5} & g_{6} & g_{7} & g_{8} \\ g_{1} & g_{2} & e & g_{4} & g_{5} & g_{3} & g_{7} & g_{8} & g_{6} \\ g_{2} & e & g_{1} & g_{5} & g_{3} & g_{4} & g_{8} & g_{6} & g_{7} \\ g_{3} & g_{4} & g_{5} & g_{6} & g_{7} & g_{8} & e & g_{1} & g_{2} \\ g_{4} & g_{5} & g_{3} & g_{7} & g_{8} & g_{6} & g_{1} & g_{2} & e \\ g_{5} & g_{3} & g_{4} & g_{8} & g_{6} & g_{7} & g_{2} & e & g_{1} \\ g_{6} & g_{7} & g_{8} & e & g_{1} & g_{2} & g_{3} & g_{4} & g_{5} \\ g_{7} & g_{8} & g_{6} & g_{1} & g_{2} & e & g_{4} & g_{5} & g_{3} \\ g_{8} & g_{6} & g_{7} & g_{2} & e & g_{1} & g_{5} & g_{3} & g_{4}\end{array}$

To find the canonical naming we first observe that the group is commutative. Our group naming will be defined if we choose any two objects $a, b$ such that $a^{2} \neq b$. Let $e=8$, and choose an object $a=7$. Whatever our choice of $a$, we will have to make $a^{2}=6$. This maximizes our representation so far.

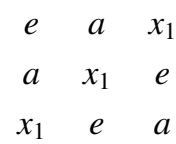

Now we need to choose a second object, $b=5$, then we have to name $a * b=x_{2}=4$ and $a * x_{2}=3$.

$\begin{array}{cccccc}e & a & x_{1} & b & x_{2} & x_{3} \\ a & x_{1} & e & x_{2} & x_{3} & b \\ x_{1} & e & a & x_{3} & b & x_{2} \\ b & x_{2} & x_{3} & & & \\ x_{2} & x_{3} & b & & & \\ x_{3} & b & x_{2} & & & \end{array}$

Whatever choice of $a, b$ we make, we will have $b^{2}$ equal to a new object $c=2$.

$\begin{array}{ccccccccc}e & a & x_{1} & b & x_{2} & x_{3} & c & x_{4} & x_{5} \\ a & x_{1} & e & x_{2} & x_{3} & b & & & \\ x_{1} & e & a & x_{3} & b & x_{2} & & & \\ b & x_{2} & x_{3} & c & & & & \\ x_{2} & x_{3} & b & & & & & \\ x_{3} & b & x_{2} & & & & & \\ c & x_{4} & & & & & & \\ x_{4} & x_{5} & & & & & & \\ x_{5} & c & & & & & & \end{array}$

This has determined our naming function. We simply have to choose two objects $a, b$ such that $a^{2} \neq b$ and the rest of the naming values are determined. Any object of the group can be chosen as $a=7$, therefore, all objects are equivalent. An 
example of a canonical naming function is $e=8, g_{8}=a=7, g_{4}=x_{1}=6, g_{7}=b=5, g_{3}=x_{2}=4, g_{2}=x_{3}=3, g_{5}=c=2$, $g_{1}=x_{4}=1, g_{6}=x_{5}=0$. This gives us the numeric table

$\begin{array}{lllllllll}8 & 7 & 6 & 5 & 4 & 3 & 2 & 1 & 0 \\ 7 & 6 & 8 & 4 & 3 & 5 & 1 & 0 & 2 \\ 6 & 8 & 7 & 3 & 5 & 4 & 0 & 2 & 1 \\ 5 & 4 & 3 & 2 & 1 & 0 & 8 & 7 & 6 \\ 4 & 3 & 5 & 1 & 0 & 2 & 7 & 6 & 8 \\ 3 & 5 & 4 & 0 & 2 & 1 & 6 & 8 & 7 \\ 2 & 1 & 0 & 8 & 7 & 6 & 5 & 4 & 3 \\ 1 & 0 & 2 & 7 & 6 & 8 & 4 & 3 & 5 \\ 0 & 2 & 1 & 6 & 8 & 7 & 3 & 5 & 4\end{array}$

The canonical representation of this group is the number

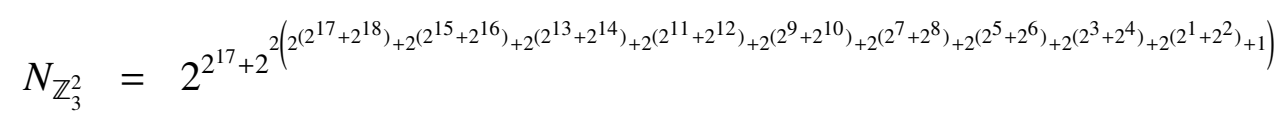

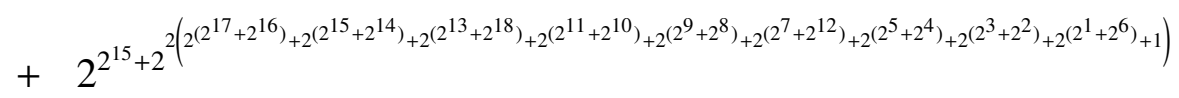

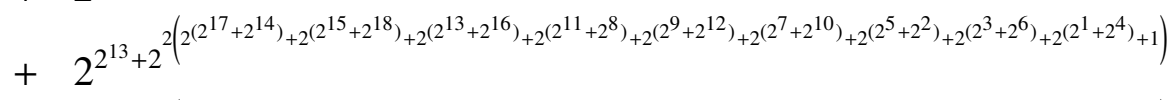

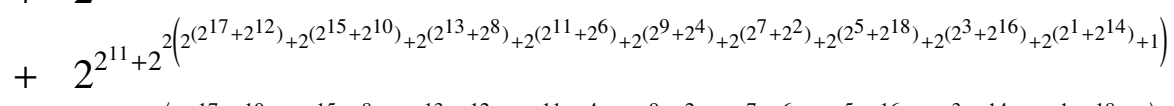

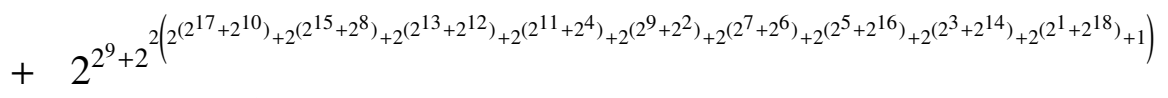

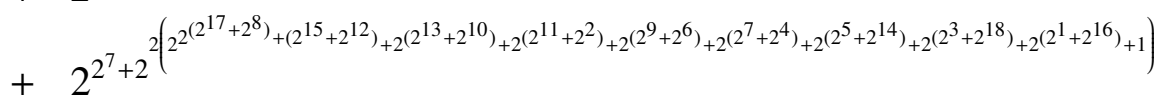

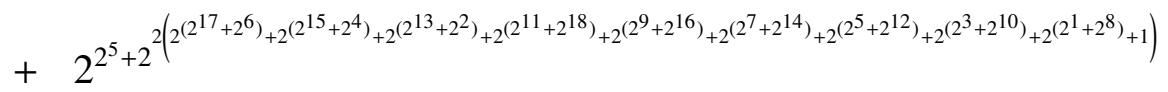

$$
\begin{aligned}
& +2^{\left.2^{3}+2^{\left.2\left(2^{\left(2^{17}\right.}+2^{4}\right)+2^{\left(2^{15}\right.}+2^{2}\right)}+2^{\left(2^{13}+2^{6}\right)}+2^{\left(2^{11}+2^{16}\right)}+2^{\left(2^{9}+2^{14}\right)}+2^{\left(2^{7}+2^{18}\right)}+2^{\left(2^{5}+2^{10}\right)}+2^{\left(2^{3}+2^{8}\right)}+2^{\left(2^{1}+2^{12}\right)}+1\right)}
\end{aligned}
$$

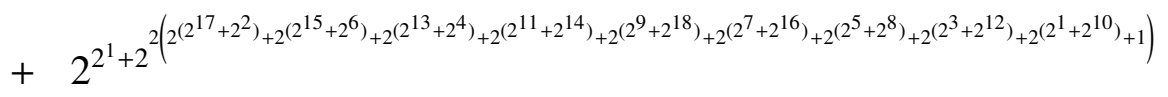

Cyclic Group $\mathbb{Z}_{9}$. If $|G|=9$, we know the objects of $G$ have order 3 or 9 . We found the only group with all the objects if third order. Now we consider the case where we have at least one object of order 9. But, since our group has nine objects, this must be the cyclic group, $\mathbb{Z}_{9}$. The cyclic group of nine objects is trivially given by the numeric table 


$\begin{array}{lllllllll}8 & 7 & 6 & 5 & 4 & 3 & 2 & 1 & 0 \\ 7 & 6 & 5 & 4 & 3 & 2 & 1 & 0 & 8 \\ 6 & 5 & 4 & 3 & 2 & 1 & 0 & 8 & 7 \\ 5 & 4 & 3 & 2 & 1 & 0 & 8 & 7 & 6 \\ 4 & 3 & 2 & 1 & 0 & 8 & 7 & 6 & 5 \\ 3 & 2 & 1 & 0 & 8 & 7 & 6 & 5 & 4 \\ 2 & 1 & 0 & 8 & 7 & 6 & 5 & 4 & 3 \\ 1 & 0 & 8 & 7 & 6 & 5 & 4 & 3 & 2 \\ 0 & 8 & 7 & 6 & 5 & 4 & 3 & 2 & 1\end{array}$

The canonical representation is

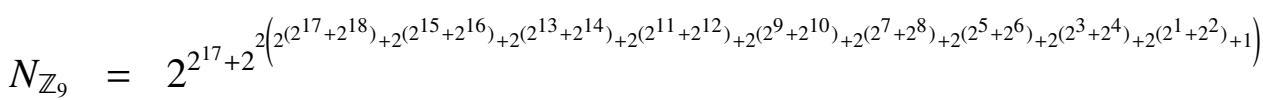

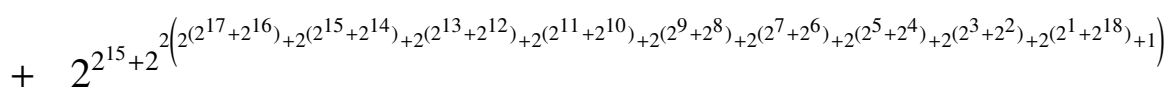

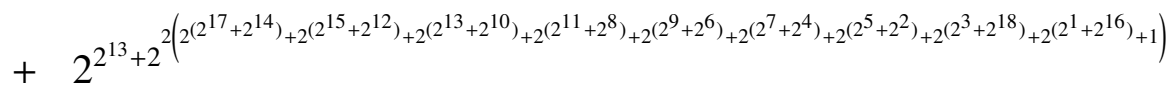

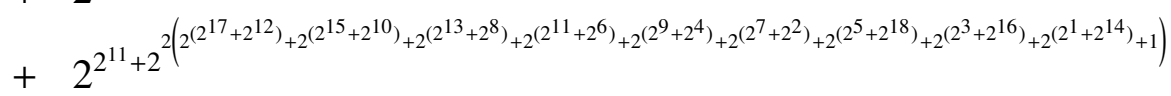

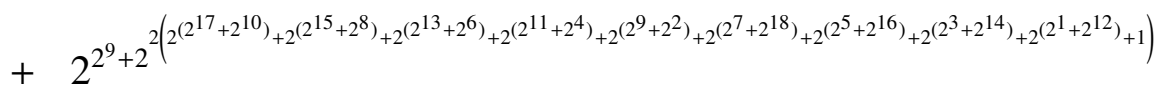

$$
\begin{aligned}
& +2^{\left.2^{7}+2^{2\left(2^{\left(2^{17}+2^{8}\right)}+\left(2^{15}+2^{6}\right)\right.}+2^{\left(2^{13}+2^{4}\right)}+2^{\left(2^{11}+2^{2}\right)}+2^{\left(2^{9}+2^{18}\right)}+2^{\left(2^{7}+2^{16}\right)}+2^{\left(2^{5}+2^{14}\right)}+2^{\left(2^{3}+2^{12}\right)}+2^{\left(2^{1}+2^{10}\right)}+1\right)}
\end{aligned}
$$

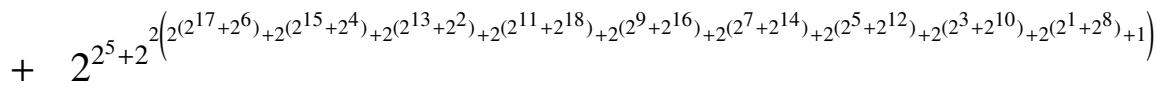

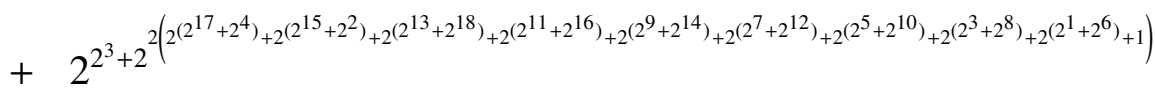

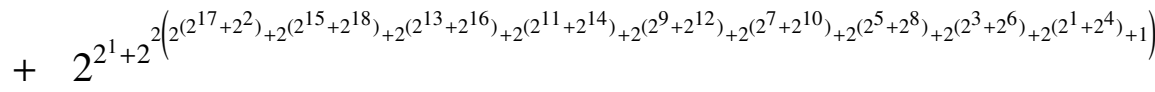

Comparing these two groups of nine elements, we verify $\mathbb{Z}_{9}<\mathbb{Z}_{3}^{2}$.

It is becoming more clear how to find the canonical representation, without having to calculate all the representations. But we still have several steps before considering the general case. 


\section{$4.7 \quad \Delta_{4}$}

Let us exhibit, for reference, the multiplication table of $\Delta_{4}$. The symbols $g_{i}$ are used for the elements of order 2 . We use $h_{i}$ for the rest of the objects.

\begin{tabular}{|c|c|c|c|c|c|c|c|c|c|c|c|c|c|c|c|c|c|c|c|c|c|c|c|}
\hline & B1 1 & 2 & 3 & 4 & $g_{5}$ & 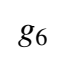 & 77 & 50 & 39 & & $z$ & & $h_{2}$ & $h_{5}$ & 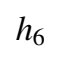 & 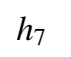 & & $h_{9}$ & 10 & 11 & 12 & & 14 \\
\hline$g_{1}$ & $e$ & $g_{4}$ & $g_{5}$ & $g_{2}$ & $g_{3}$ & $h_{2}$ & $h_{1}$ & $h_{4}$ & $h_{3}$ & $g_{7}$ & $g_{6}$ & $g_{9}$ & $g_{8}$ & $h_{6}$ & 75 & $h_{12}$ & 11 & $h_{14}$ & $h_{13}$ & $h_{8}$ & $h_{7}$ & 10 & $h_{9}$ \\
\hline 92 & $g_{4}$ & 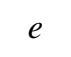 & $h_{6}$ & $g_{1}$ & $h_{5}$ & $h_{1}$ & $h_{2}$ & 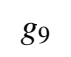 & $g_{8}$ & $g_{6}$ & $g_{7}$ & & & $g_{5}$ & $g_{3}$ & $h_{11}$ & & $h_{10}$ & l & 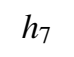 & 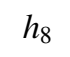 & 14 & l \\
\hline & $g_{5}$ & $h_{5}$ & $e$ & $n_{0}$ & $g_{1}$ & $h_{10}$ & $h_{9}$ & $h_{8}$ & $h_{7}$ & $h_{14}$ & $h_{13}$ & 12 & $h_{11}$ & & & 8 & & 37 & 36 & $h_{4}$ & 3 & $t_{2}$ & $h_{1}$ \\
\hline & $g_{2}$ & $g_{1}$ & $h_{5}$ & $e$ & $h_{6}$ & $g_{7}$ & $g_{6}$ & $h_{3}$ & $h_{4}$ & $h_{2}$ & $h_{1}$ & $g_{8}$ & $g_{9}$ & $g_{3}$ & 35 & $h_{8}$ & $i_{7}$ & $h_{13}$ & $h_{14}$ & $h_{12}$ & $h_{11}$ & $h_{9}$ & 10 \\
\hline & $g_{3}$ & $h_{6}$ & $g_{1}$ & $h_{5}$ & $e$ & $h_{13}$ & $h_{14}$ & $h_{11}$ & $h_{12}$ & $h_{9}$ & $h_{10}$ & 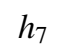 & $h_{8}$ & & 2 & $h_{3}$ & 4 & $h_{1}$ & $h_{2}$ & 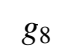 & 99 & 96 & \\
\hline & $h_{1}$ & $h_{2}$ & $h_{7}$ & $g_{7}$ & $h_{11}$ & $e$ & $g_{4}$ & $h_{13}$ & 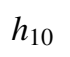 & $g_{1}$ & $g_{2}$ & $h_{9}$ & $h_{14}$ & $h_{8}$ & 12 & $g_{3}$ & $h_{5}$ & $h_{3}$ & $g_{9}$ & & $h_{6}$ & 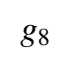 & \\
\hline & $h_{2}$ & $h_{1}$ & $h_{8}$ & $g_{6}$ & $h_{12}$ & $g_{4}$ & $e$ & $h_{9}$ & $h_{14}$ & $g_{2}$ & $g_{1}$ & $h_{13}$ & $h_{10}$ & $n_{7}$ & $h_{11}$ & $h_{5}$ & $g_{3}$ & $g_{8}$ & $h_{4}$ & $h_{6}$ & $g_{5}$ & $h_{3}$ & \\
\hline & $h_{3}$ & $g_{9}$ & $h_{9}$ & $h_{4}$ & $h_{13}$ & $h_{11}$ & $h_{8}$ & $e$ & $g_{2}$ & $h_{7}$ & $h_{12}$ & $g_{1}$ & $g_{4}$ & $h_{14}$ & $h_{10}$ & $h_{1}$ & 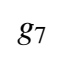 & 5 & $h_{6}$ & 6 & $l_{2}$ & $g_{5}$ & \\
\hline & $h_{4}$ & $g_{8}$ & $h_{1}$ & $h_{3}$ & $h_{u}$ & $h_{7}$ & $h_{12}$ & $g_{2}$ & 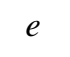 & $h_{11}$ & $h_{8}$ & $g_{4}$ & & & $h_{9}$ & & & 6 & & 1 & & & \\
\hline$\eta$ & $g_{6}$ & $g_{7}$ & $h_{11}$ & $h_{2}$ & $h_{7}$ & $g_{2}$ & $g_{1}$ & $h_{14}$ & $h_{9}$ & $g_{4}$ & $e$ & $h_{10}$ & $h_{13}$ & $h_{12}$ & $h_{8}$ & $h_{6}$ & $g_{5}$ & $h_{4}$ & $g_{8}$ & $h_{5}$ & $g_{3}$ & 99 & \\
\hline$h_{2}$ & $g_{7}$ & $g_{6}$ & $h_{12}$ & $h_{1}$ & $h_{8}$ & $g_{1}$ & $g_{2}$ & $h_{10}$ & $h_{13}$ & 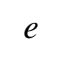 & $g_{4}$ & $h_{14}$ & $h_{9}$ & $h_{11}$ & $h_{7}$ & $g_{5}$ & $h_{6}$ & $g_{9}$ & $h_{3}$ & 3 & 15 & $n_{4}$ & \\
\hline$\eta$ & $g_{8}$ & $h_{4}$ & $h_{13}$ & $g_{9}$ & $h_{9}$ & $h_{12}$ & $h_{7}$ & $g_{4}$ & $g_{1}$ & $h_{8}$ & $h_{11}$ & $g_{2}$ & $e$ & $h_{10}$ & $h_{14}$ & $h_{2}$ & $g_{6}$ & $h_{5}$ & $g_{5}$ & 1 & $h_{1}$ & $h_{6}$ & \\
\hline$\eta$ & $g_{9}$ & $h_{3}$ & $h_{14}$ & $g_{8}$ & $h_{10}$ & $h_{8}$ & $h_{11}$ & $g_{1}$ & $g_{4}$ & $h_{12}$ & $h_{7}$ & $e$ & $g_{2}$ & $h_{9}$ & $h_{13}$ & $g_{7}$ & $h_{1}$ & $g_{5}$ & $h_{5}$ & $h_{2}$ & $g_{6}$ & $g_{3}$ & \\
\hline$h$ & $h_{6}$ & $g_{3}$ & $g_{4}$ & $g_{5}$ & $g_{2}$ & $h_{14}$ & $h_{13}$ & $h_{7}$ & $h_{8}$ & $h_{10}$ & $h_{9}$ & $h_{11}$ & $h_{12}$ & $g_{1}$ & 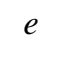 & $h_{4}$ & $h_{3}$ & 6 & t & & $g_{8}$ & ${ }_{1}$ & \\
\hline$h_{6}$ & $h_{5}$ & $g_{5}$ & $g_{2}$ & 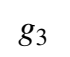 & $g$ & $h_{9}$ & $h_{10}$ & $h_{12}$ & $h_{11}$ & $h_{13}$ & $h_{14}$ & $h_{8}$ & $h_{7}$ & $e$ & $g_{1}$ & $g_{8}$ & $g_{9}$ & $h_{2}$ & $h_{1}$ & $h_{3}$ & $h_{4}$ & $g_{7}$ & \\
\hline$h_{7}$ & $h_{11}$ & $h_{8}$ & $g_{6}$ & $h_{12}$ & $h_{1}$ & $g_{9}$ & $h_{3}$ & $h_{5}$ & $g_{3}$ & $h_{4}$ & $g_{8}$ & $h_{6}$ & $g_{5}$ & $h_{2}$ & $g_{7}$ & $h_{10}$ & $h_{13}$ & $g_{4}$ & $e$ & $h_{14}$ & $h_{9}$ & 2 & \\
\hline & $h_{12}$ & $h_{7}$ & $g_{7}$ & $h_{11}$ & $h_{2}$ & $h_{4}$ & $g_{8}$ & $g_{3}$ & $h_{5}$ & $g_{9}$ & $h_{3}$ & $g_{5}$ & $h_{6}$ & $h_{1}$ & & $h_{14}$ & $h_{9}$ & $e$ & $g_{4}$ & $h_{10}$ & $h_{13}$ & 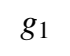 & \\
\hline$h_{9}$ & $h_{13}$ & $h_{14}$ & $g_{8}$ & $h_{10}$ & $h_{3}$ & $h_{6}$ & $g_{3}$ & $g_{7}$ & $h_{1}$ & $h_{5}$ & $g_{5}$ & $h_{2}$ & or & $g_{9}$ & $h_{4}$ & $g_{2}$ & $e$ & $h_{8}$ & $h_{11}$ & & $g_{1}$ & $h_{12}$ & \\
\hline & $h_{14}$ & $h_{13}$ & $g_{9}$ & $h_{9}$ & $h_{4}$ & $g_{3}$ & $h_{6}$ & $h_{2}$ & $g_{6}$ & $g_{5}$ & $h_{5}$ & $g_{7}$ & $h_{1}$ & $g_{8}$ & $h_{3}$ & $e$ & $g_{2}$ & $h_{12}$ & $h_{7}$ & 1 & $g_{4}$ & $h_{8}$ & $t_{1}$ \\
\hline & $h_{7}$ & $h_{12}$ & $h_{1}$ & $h_{8}$ & $g_{\epsilon}$ & $g_{8}$ & $h_{4}$ & $g_{5}$ & $h_{6}$ & $h$ & $g_{9}$ & 05 & $h_{5}$ & $g$ & 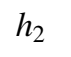 & $h_{9}$ & $h_{14}$ & $g_{1}$ & $g_{2}$ & $h_{13}$ & $h_{10}$ & & \\
\hline & $h_{8}$ & $h_{11}$ & $h_{2}$ & $h_{7}$ & $g_{7}$ & $h_{3}$ & $g_{9}$ & $h_{6}$ & $g_{5}$ & & $h_{4}$ & $h_{5}$ & 03 & 00 & $h_{1}$ & $h_{13}$ & $h_{10}$ & & $g_{1}$ & $h_{9}$ & $h_{14}$ & 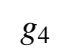 & \\
\hline & $h_{9}$ & $h_{10}$ & $h_{3}$ & $h_{14}$ & $g_{8}$ & $g_{5}$ & $h_{5}$ & $g_{6}$ & $h_{2}$ & & $h_{6}$ & $h_{1}$ & $g_{7}$ & $h_{4}$ & $g_{9}$ & $g_{1}$ & $g_{4}$ & $h_{7}$ & $h_{12}$ & & $g_{2}$ & $h_{11}$ & \\
\hline & $h_{10}$ & $h_{9}$ & $h_{4}$ & $h_{13}$ & $g_{9}$ & $h_{5}$ & $g_{5}$ & $h_{1}$ & $g_{7}$ & ${ }^{6} 6$ & $g_{3}$ & $g_{6}$ & $h_{2}$ & $h_{3}$ & $g_{8}$ & $g_{4}$ & $g_{1}$ & $h_{11}$ & $h_{8}$ & 2 & $e$ & $h_{7}$ & \\
\hline
\end{tabular}

We do mention, ahead of time, the canonical naming function does not assign the highest ten values to the set $\left\{e, g_{1}, g_{2}, \ldots, g_{9}\right\}$. Some $h_{i}$ objects will have a higher numeric value than some $g_{i}$. The smallest order of any non trivial object is 2 . It is obvious we must make $e=23,23=a, 22=b$ for two second order objects, $a, b$, that commute. We have the following possible pairs:

$\begin{array}{ll}\left\{g_{1}, g_{2}\right\} & \left\{g_{4}, g_{6}\right\} \\ \left\{g_{1}, g_{4}\right\} & \left\{g_{4}, g_{7}\right\} \\ \left\{g_{2}, g_{4}\right\} & \left\{g_{6}, g_{7}\right\} \\ \left\{g_{1}, g_{3}\right\} & \left\{g_{2}, g_{8}\right\} \\ \left\{g_{1}, g_{5}\right\} & \left\{g_{2}, g_{9}\right\} \\ \left\{g_{3}, g_{5}\right\} & \left\{g_{8}, g_{9}\right\}\end{array}$

Let $a, b$ any of these pairs; we can use the pairs in either order because they are not ordered pairs. For example, we can have $a=g_{1}, b=g_{2}$, or we can also assign $a=g_{2}, b=g_{1}$. We can use any of the pairs above, and the naming function $e=23$, $a=22, b=21, x_{1}=20$ gives the table 


$\begin{array}{cccc}e & a & b & x_{1} \\ a & e & x_{1} & b \\ b & x_{1} & e & a \\ x_{1} & b & a & e\end{array}$

If we wish to maximize our representation, we shall find $a, b, x_{1}$ such that $\left\{e, a, b, x_{1}\right\}$ forms the Klein 4-group. We see that in fact the triads

$$
\left\{g_{1}, g_{2}, g_{4}\right\} \quad\left\{g_{1}, g_{3}, g_{5}\right\} \quad\left\{g_{4}, g_{6}, g_{7}\right\} \quad\left\{g_{2}, g_{8}, g_{9}\right\}
$$

form the Klein 4-group. Given any one of these triads, we do not know which objects will be $a$ and $b$. For example, if we work with $\left\{g_{1}, g_{2}, g_{4}\right\}$, who should we define as $a, b, x_{1}$ ? All the non trivial objects of $K(4)$ are equivalent, so we can not decide upon this yet. Let us add a new object $c_{1}$ and $x_{2}=a * c_{1}$

$\begin{array}{cccccc}e & a & b & x_{1} & c_{1} & x_{2} \\ a & e & x_{1} & b & & \\ b & x_{1} & e & a & & \\ x_{1} & b & a & e & & \\ c_{1} & x_{2} & & & & \\ x_{2} & c_{1} & & & & \end{array}$

We also need a new object $c_{2}=b * c_{1}$, and $x_{3}=a * c_{2}$.

$\begin{array}{cccccccc}e & a & b & x_{1} & c_{1} & x_{2} & c_{2} & x_{3} \\ a & e & x_{1} & b & & & & \\ b & x_{1} & e & a & & & & \\ x_{1} & b & a & e & & & & \\ c_{1} & x_{2} & c_{2} & x_{3} & & & & \\ x_{2} & c_{1} & x_{3} & c_{2} & & & & \\ c_{2} & x_{3} & c_{1} & x_{2} & & & & \\ x_{3} & c_{2} & x_{2} & c_{1} & & & & \end{array}$

In summary, the canonical naming function will involve one of the Klein 4-subgroups. And, we need an object $c_{1}$ that commutes with $a$, if it should exist. This maximizes the last table. We will see that there are still several options to do this. In fact, all our candidate naming functions admit an object $c_{1}$ that commutes with $a$. This gives the table

$$
\begin{array}{llllllll}
e & a & b & x_{1} & c_{1} & x_{2} & c_{2} & x_{3} \\
a & e & x_{1} & b & x_{2} & c_{1} & & \\
b & x_{1} & e & a & & & & \\
x_{1} & b & a & e & & & & \\
c_{1} & x_{2} & c_{2} & x_{3} & & & & \\
x_{2} & c_{1} & x_{3} & c_{2} & & & \\
c_{2} & x_{3} & c_{1} & x_{2} & & & \\
x_{3} & c_{2} & x_{2} & c_{1} & & & &
\end{array}
$$


determined by the equations

$$
\begin{aligned}
e & =a^{2}=b^{2} \\
a * b & =b * a \\
a * c_{1} & =c_{1} * a .
\end{aligned}
$$

For the triads in 16 , we need to find an object $c_{1}$ that commutes with $a$. For example, all the objects in $H_{1}=\left\{g_{1}, g_{2}, g_{4}\right\}$ commute with at least one object not in $H_{1}$. In the case of $H_{2}=\left\{g_{2}, g_{8}, g_{9}\right\}$, only $g_{2}$ commutes with objects not in $H_{2}$. This means if we are working with the triad $\left\{g_{2}, g_{8}, g_{9}\right\}$ we must have $a=g_{2}$. For $\left\{g_{1}, g_{3}, g_{5}\right\}$ we must have $a=g_{1}$, and for $\left\{g_{4}, g_{6}, g_{7}\right\}$ we must assign $a=g_{4}$. We list the objects that commute with each second order object $g_{i}$.

$$
\begin{array}{ccc}
\operatorname{Comm}\left(g_{1}\right)=\left\{g_{2}, g_{4}, g_{3}, g_{5}, h_{5}, h_{6}\right\} & \operatorname{Comm}\left(g_{2}\right)=\left\{g_{1}, g_{4}, g_{8}, g_{9}, h_{3}, h_{4}\right\} & \operatorname{Comm}\left(g_{3}\right)=\left\{g_{1}, g_{5}\right\} \\
\operatorname{Comm}\left(g_{4}\right)=\left\{g_{1}, g_{2}, g_{6}, g_{7}, h_{1}, h_{2}\right\} & \operatorname{Comm}\left(g_{5}\right)=\left\{g_{1}, g_{3}\right\} & \operatorname{Comm}\left(g_{6}\right)=\left\{g_{4}, g_{7}\right\} \\
\operatorname{Comm}\left(g_{7}\right)=\left\{g_{4}, g_{6}\right\} & \operatorname{Comm}\left(g_{8}\right)=\left\{g_{2}, g_{9}\right\} & \operatorname{Comm}\left(g_{9}\right)=\left\{g_{2}, g_{8}\right\}
\end{array}
$$

This information reduces our possible naming functions because now we know a little more about $a$. Our possible naming functions are more than we would like to list, but they are easy to describe. We need an object $a \in\left\{g_{1}, g_{2}, g_{4}\right\}$, and we need a second order object $b$ to determine the subgroup $K(4)$. For example, if we choose $a=g_{4}$ we can choose $b \in\left\{g_{1}, g_{2}, g_{6}, g_{7}\right\}$; find a second order object that commutes with $a=g_{4}$. In the case of $a=g_{1}$ we need to choose $b \in\left\{g_{2}, g_{4}, g_{3}, g_{5}\right\}$. If $a=g_{2}$ then $b \in\left\{g_{1}, g_{4}, g_{8}, g_{9}\right\}$. After determining the subgroup $K(4)$, we need an object $c_{1}$ that commutes with $a$. The expressions of (17) let us know which combinations allow $c_{1}$. We shall start representing naming functions with finite sequences. We will write naming functions in the form $\left(a, b, x_{1}, c_{1}, x_{2}, c_{2}, x_{3}\right)$. For example, the naming function $a=g_{4}, b=g_{2}, x_{1}=a * b=g_{1}$, $c_{1}=g_{7}, x_{2}=a * c_{1}=g_{6}, c_{2}=b * c_{1}=h_{1}, x_{3}=a * c_{2}=h_{2}$ is given by the expression $\left(g_{4}, g_{2}, g_{1}, g_{7}, g_{6}, h_{1}, h_{2}\right)$. We know $a \in g_{1}, g_{2}, g_{4}$ for any of the triads giving $K(4)$. Choose a second order object, $b$, that commutes with $a$, and then we choose an object $c_{1}$ that also commutes with $a$. We list all possible naming functions below.

$$
\begin{aligned}
& \left(g_{1}, g_{2}, g_{4}, g_{3}, g_{5}, h_{5}, h_{6}\right) \\
& \left(g_{2}, g_{1}, g_{4}, g_{8}, g_{9}, h_{3}, h_{4}\right) \\
& \left(g_{4}, g_{1}, g_{2}, g_{6}, g_{7}, h_{1}, h_{2}\right) \\
& \left(g_{1}, g_{2}, g_{4}, g_{5}, g_{3}, h_{6}, h_{5}\right) \\
& \left(g_{2}, g_{1}, g_{4}, g_{9}, g_{8}, h_{4}, h_{3}\right) \\
& \left(g_{4}, g_{1}, g_{2}, g_{7}, g_{6}, h_{2}, h_{1}\right) \\
& \left(g_{1}, g_{2}, g_{4}, h_{5}, h_{6}, g_{3}, g_{5}\right) \\
& \left(g_{2}, g_{1}, g_{4}, h_{3}, h_{4}, g_{8}, g_{9}\right) \\
& \left(g_{4}, g_{1}, g_{2}, h_{1}, h_{2}, g_{6}, g_{7}\right) \\
& \left(g_{1}, g_{2}, g_{4}, h_{6}, h_{5}, g_{5}, g_{3}\right) \\
& \left(g_{2}, g_{1}, g_{4}, h_{4}, h_{3}, g_{9}, g_{8}\right) \\
& \left(g_{4}, g_{1}, g_{2}, h_{2}, h_{1}, g_{7}, g_{6}\right) \\
& \left(g_{1}, g_{4}, g_{2}, g_{3}, g_{5}, h_{6}, h_{5}\right) \\
& \left(g_{2}, g_{4}, g_{1}, g_{8}, g_{9}, h_{4}, h_{3}\right) \\
& \left(g_{4}, g_{2}, g_{1}, g_{6}, g_{7}, h_{2}, h_{1}\right) \\
& \left(g_{1}, g_{4}, g_{2}, g_{5}, g_{3}, h_{5}, h_{6}\right) \\
& \left(g_{2}, g_{4}, g_{1}, g_{9}, g_{8}, h_{3}, h_{4}\right) \\
& \left(g_{4}, g_{2}, g_{1}, g_{7}, g_{6}, h_{1}, h_{2}\right) \\
& \left(g_{1}, g_{4}, g_{2}, h_{5}, h_{6}, g_{5}, g_{3}\right) \\
& \left(g_{2}, g_{4}, g_{1}, h_{3}, h_{4}, g_{9}, g_{8}\right) \\
& \left(g_{4}, g_{2}, g_{1}, h_{1}, h_{2}, g_{7}, g_{6}\right) \\
& \left(g_{1}, g_{4}, g_{2}, h_{6}, h_{5}, g_{3}, g_{5}\right) \\
& \left(g_{2}, g_{4}, g_{1}, h_{4}, h_{3}, g_{8}, g_{9}\right) \\
& \left(g_{4}, g_{2}, g_{1}, h_{2}, h_{1}, g_{6}, g_{7}\right) \\
& \left(g_{1}, g_{3}, g_{5}, g_{2}, g_{4}, h_{6}, h_{5}\right) \\
& \left(g_{2}, g_{8}, g_{9}, g_{1}, g_{4}, h_{4}, h_{3}\right) \\
& \left(g_{4}, g_{6}, g_{7}, g_{1}, g_{2}, h_{2}, h_{1}\right) \\
& \left(g_{1}, g_{3}, g_{5}, g_{4}, g_{2}, h_{5}, h_{6}\right) \\
& \left(g_{2}, g_{8}, g_{9}, g_{4}, g_{1}, h_{3}, h_{4}\right) \\
& \left(g_{4}, g_{6}, g_{7}, g_{2}, g_{1}, h_{1}, h_{2}\right) \\
& \left(g_{1}, g_{3}, g_{5}, h_{5}, h_{6}, g_{4}, g_{2}\right) \\
& \left(g_{2}, g_{8}, g_{9}, h_{3}, h_{4}, g_{4}, g_{1}\right) \\
& \left(g_{4}, g_{6}, g_{7}, h_{1}, h_{2}, g_{2}, g_{1}\right) \\
& \left(g_{1}, g_{3}, g_{5}, h_{6}, h_{5}, g_{2}, g_{4}\right) \\
& \left(g_{2}, g_{8}, g_{9}, h_{4}, h_{3}, g_{1}, g_{4}\right) \\
& \left(g_{4}, g_{6}, g_{7}, h_{2}, h_{1}, g_{1}, g_{2}\right) \\
& \left(g_{1}, g_{5}, g_{3}, g_{2}, g_{4}, h_{5}, h_{6}\right) \\
& \left(g_{2}, g_{9}, g_{8}, g_{1}, g_{4}, h_{3}, h_{4}\right) \\
& \left(g_{4}, g_{7}, g_{6}, g_{1}, g_{4}, h_{1}, h_{2}\right) \\
& \left(g_{1}, g_{5}, g_{3}, g_{4}, g_{2}, h_{6}, h_{5}\right) \\
& \left(g_{2}, g_{9}, g_{8}, g_{4}, g_{1}, h_{4}, h_{3}\right) \\
& \left(g_{4}, g_{7}, g_{6}, g_{2}, g_{1}, h_{2}, h_{1}\right) \\
& \left(g_{1}, g_{5}, g_{3}, h_{5}, h_{6}, g_{2}, g_{4}\right) \\
& \left(g_{2}, g_{9}, g_{8}, h_{3}, h_{4}, g_{1}, g_{4}\right) \\
& \left(g_{4}, g_{7}, g_{6}, h_{1}, h_{2}, g_{1}, g_{2}\right) \\
& \left(g_{1}, g_{5}, g_{3}, h_{6}, h_{5}, g_{4}, g_{2}\right) \\
& \left(g_{2}, g_{9}, g_{8}, h_{4}, h_{3}, g_{4}, g_{1}\right)
\end{aligned}
$$


In (17) we can also observe that given any choice of $a, b, x_{1}$ that satisfies $K(4)=\left\{e, a, b, x_{1}\right\}$, there is no object $g \notin K(4)$ that commutes with both $a$ and $b$. None of our candidate triads satisfy $a * c_{1}=c_{1} * a$ and $b * c_{1}=c_{1} * b$ simultaneously. Although we can not find $c_{1}$ that commutes with $a$ and $b$, we still have to maximize our representation. The next highest object we can have in the position of $c_{1} * b$ is $x_{3}$. Each of the finite sequences above satisfies $a * c_{1}=c_{1} * a$ and $x_{3}=c_{1} * b$. Any one of our naming functions in 18 will give the table

$\begin{array}{llllllll}e & a & b & x_{1} & c_{1} & x_{2} & c_{2} & x_{3} \\ a & e & x_{1} & b & x_{2} & c_{1} & & \\ b & x_{1} & e & a & x_{3} & c_{2} & & \\ x_{1} & b & a & e & c_{2} & x_{3} & & \\ c_{1} & x_{2} & c_{2} & x_{3} & & & \\ x_{2} & c_{1} & x_{3} & c_{2} & & & \\ c_{2} & x_{3} & c_{1} & x_{2} & & & \\ x_{3} & c_{2} & x_{2} & c_{1} & & & & \end{array}$

It is possible to choose $c_{1}$ with the additional restraint $\left|c_{1}\right|=2$, maximizing the representation. The naming functions

$$
\begin{array}{lll}
\left(g_{1}, g_{2}, g_{4}, g_{3}, g_{5}, h_{5}, h_{6}\right) & \left(g_{2}, g_{1}, g_{4}, g_{8}, g_{9}, h_{3}, h_{4}\right) & \left(g_{4}, g_{1}, g_{2}, g_{6}, g_{7}, h_{1}, h_{2}\right) \\
\left(g_{1}, g_{2}, g_{4}, g_{5}, g_{3}, h_{6}, h_{5}\right) & \left(g_{2}, g_{1}, g_{4}, g_{9}, g_{8}, h_{4}, h_{3}\right) & \left(g_{4}, g_{1}, g_{2}, g_{7}, g_{6}, h_{2}, h_{1}\right) \\
\left(g_{1}, g_{4}, g_{2}, g_{3}, g_{5}, h_{6}, h_{5}\right) & \left(g_{2}, g_{4}, g_{1}, g_{8}, g_{9}, h_{4}, h_{3}\right) & \left(g_{4}, g_{2}, g_{1}, g_{6}, g_{7}, h_{2}, h_{1}\right) \\
\left(g_{1}, g_{4}, g_{2}, g_{5}, g_{3}, h_{5}, h_{6}\right) & \left(g_{2}, g_{4}, g_{1}, g_{9}, g_{8}, h_{3}, h_{4}\right) & \left(g_{4}, g_{2}, g_{1}, g_{7}, g_{6}, h_{1}, h_{2}\right) \\
\left(g_{1}, g_{3}, g_{5}, g_{2}, g_{4}, h_{6}, h_{5}\right) & \left(g_{2}, g_{8}, g_{9}, g_{1}, g_{4}, h_{4}, h_{3}\right) & \left(g_{4}, g_{6}, g_{7}, g_{1}, g_{2}, h_{2}, h_{1}\right) \\
\left(g_{1}, g_{3}, g_{5}, g_{4}, g_{2}, h_{5}, h_{6}\right) & \left(g_{2}, g_{8}, g_{9}, g_{4}, g_{1}, h_{3}, h_{4}\right) & \left(g_{4}, g_{6}, g_{7}, g_{2}, g_{1}, h_{1}, h_{2}\right) \\
\left(g_{1}, g_{5}, g_{3}, g_{2}, g_{4}, h_{5}, h_{6}\right) & \left(g_{2}, g_{9}, g_{8}, g_{1}, g_{4}, h_{3}, h_{4}\right) & \left(g_{4}, g_{7}, g_{6}, g_{1}, g_{4}, h_{1}, h_{2}\right) \\
\left(g_{1}, g_{5}, g_{3}, g_{4}, g_{2}, h_{6}, h_{5}\right) & \left(g_{2}, g_{9}, g_{8}, g_{4}, g_{1}, h_{4}, h_{3}\right) & \left(g_{4}, g_{7}, g_{6}, g_{2}, g_{1}, h_{2}, h_{1}\right)
\end{array}
$$

give the table

$\begin{array}{cccccccc}e & a & b & x_{1} & c_{1} & x_{2} & c_{2} & x_{3} \\ a & e & x_{1} & b & x_{2} & c_{1} & x_{3} & c_{2} \\ b & x_{1} & e & a & x_{3} & c_{2} & x_{2} & c_{1} \\ x_{1} & b & a & e & c_{2} & x_{3} & c_{1} & x_{2} \\ c_{1} & x_{2} & c_{2} & x_{3} & e & a & b & x_{1} \\ x_{2} & c_{1} & x_{3} & c_{2} & a & e & x_{1} & b \\ c_{2} & x_{3} & c_{1} & x_{2} & x_{1} & b & a & e \\ x_{3} & c_{2} & x_{2} & c_{1} & b & x_{1} & e & a\end{array}$

This is the table obtained for the canonical naming of the Dihedral Group $D_{8}$. We add a new object $d_{1}$ and $x_{4}=a * d_{1}$, $d_{2}=b * d_{1}, x_{5}=a * d_{2}$ to the table above. 


$\begin{array}{llllllllllll}e & a & b & x_{1} & c_{1} & x_{2} & c_{2} & x_{3} & d_{1} & x_{4} & d_{2} & x_{5} \\ a & e & x_{1} & b & x_{2} & c_{1} & x_{3} & c_{2} & & & & \\ b & x_{1} & e & a & x_{3} & c_{2} & x_{2} & c_{1} & & & & \\ x_{1} & b & a & e & c_{2} & x_{3} & c_{1} & x_{2} & & & \\ c_{1} & x_{2} & c_{2} & x_{3} & e & a & b & x_{1} & & & \\ x_{2} & c_{1} & x_{3} & c_{2} & a & e & x_{1} & b & & & \\ c_{2} & x_{3} & c_{1} & x_{2} & x_{1} & b & a & e & & & \\ x_{3} & c_{2} & x_{2} & c_{1} & b & x_{1} & e & a & & \\ d_{1} & x_{4} & d_{2} & x_{5} & & & & & & \\ x_{4} & d_{1} & x_{5} & d_{2} & & & & & & & \\ d_{2} & x_{5} & d_{1} & x_{4} & & & & & & & \\ x_{5} & d_{2} & x_{4} & d_{1} & & & & & & & \end{array}$

We find we have to add another new object $p_{1}=c_{1} * d_{1}$. Then, we have to add the objects $x_{6}=a * p_{1}, p_{2}=b * p_{1}, x_{7}=a * p_{2}$.

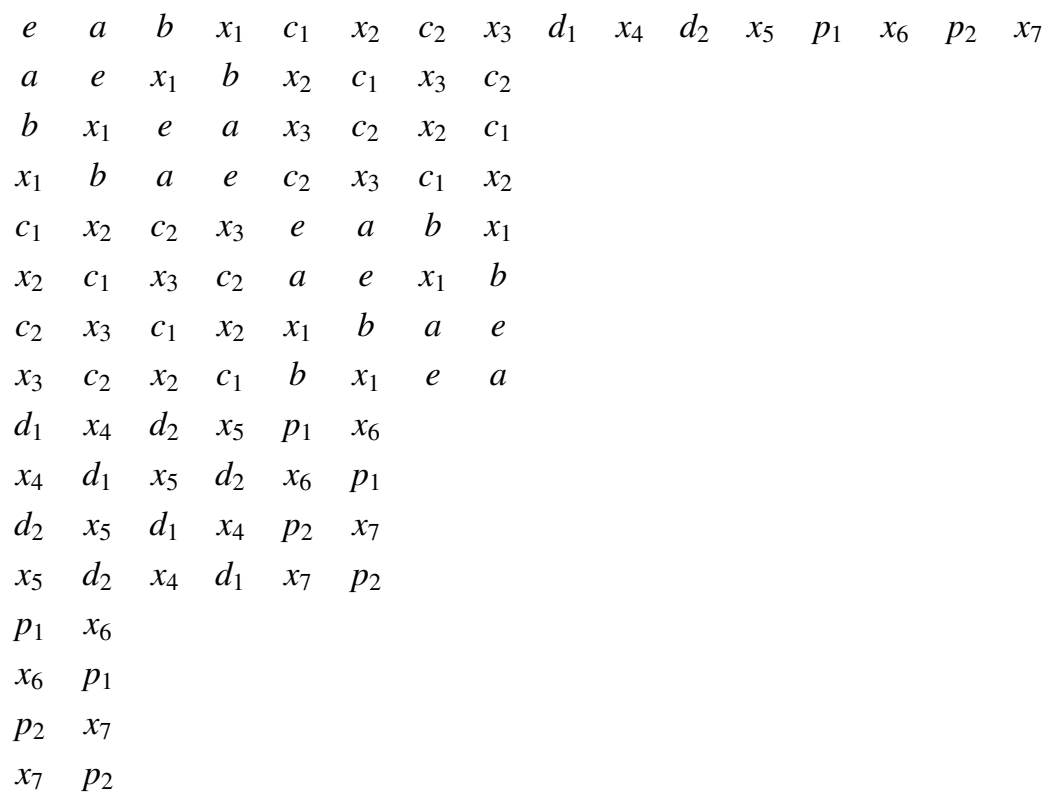

Use $\left|c_{1}\right|=2$ to find $c_{1} * p_{1}=d_{1}$, etc. It is not hard to find $c_{2} * d_{1}=x_{7}$. 


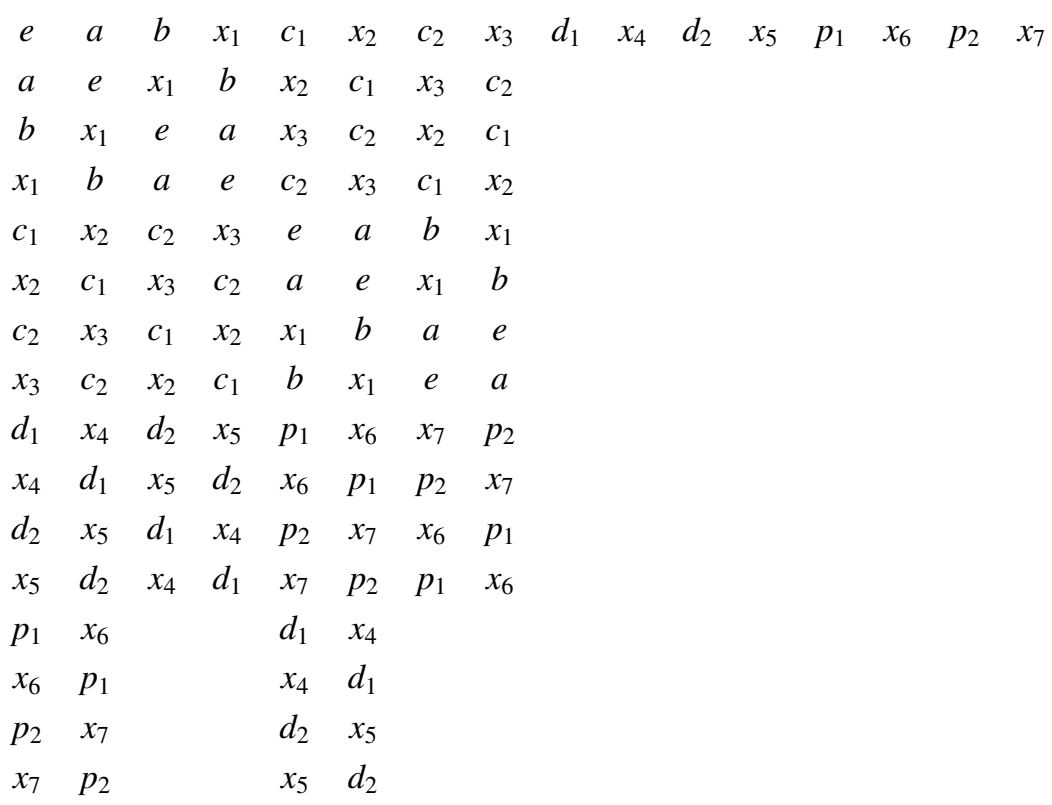

Now we can use $b=c_{2} * c_{1}$ to find $b * p_{1}=x_{7}$. We similarly find $b * p_{2}=x_{6}$. Finding $c_{2} * p_{1}=d_{2}$ is easy using $c_{2}=c_{1} * x_{1}$.

$$
\begin{aligned}
& \begin{array}{llllllllllllllll}
e & a & b & x_{1} & c_{1} & x_{2} & c_{2} & x_{3} & d_{1} & x_{4} & d_{2} & x_{5} & p_{1} & x_{6} & p_{2} & x_{7}
\end{array} \\
& \begin{array}{llllllll}
a & e & x_{1} & b & x_{2} & c_{1} & x_{3} & c_{2}
\end{array} \\
& \begin{array}{llllllll}
b & x_{1} & e & a & x_{3} & c_{2} & x_{2} & c_{1}
\end{array} \\
& \begin{array}{llllllll}
x_{1} & b & a & e & c_{2} & x_{3} & c_{1} & x_{2}
\end{array} \\
& \begin{array}{llllllll}
c_{1} & x_{2} & c_{2} & x_{3} & e & a & b & x_{1}
\end{array} \\
& \begin{array}{llllllll}
x_{2} & c_{1} & x_{3} & c_{2} & a & e & x_{1} & b
\end{array} \\
& \begin{array}{llllllll}
c_{2} & x_{3} & c_{1} & x_{2} & x_{1} & b & a & e
\end{array} \\
& \begin{array}{llllllll}
x_{3} & c_{2} & x_{2} & c_{1} & b & x_{1} & e & a
\end{array} \\
& \begin{array}{llllllll}
d_{1} & x_{4} & d_{2} & x_{5} & p_{1} & x_{6} & x_{7} & p_{2}
\end{array} \\
& \begin{array}{llllllll}
x_{4} & d_{1} & x_{5} & d_{2} & x_{6} & p_{1} & p_{2} & x_{7}
\end{array} \\
& \begin{array}{llllllll}
d_{2} & x_{5} & d_{1} & x_{4} & p_{2} & x_{7} & x_{6} & p_{1}
\end{array} \\
& \begin{array}{llllllll}
x_{5} & d_{2} & x_{4} & d_{1} & x_{7} & p_{2} & p_{1} & x_{6}
\end{array} \\
& \begin{array}{llllllll}
p_{1} & x_{6} & x_{7} & p_{2} & d_{1} & x_{4} & d_{2} & x_{5}
\end{array} \\
& \begin{array}{llllllll}
x_{6} & p_{1} & p_{2} & x_{7} & x_{4} & d_{1} & x_{5} & d_{2}
\end{array} \\
& \begin{array}{lllllllll}
p_{2} & x_{7} & x_{6} & p_{1} & d_{2} & x_{5} & d_{1} & x_{4}
\end{array} \\
& \begin{array}{llllllll}
x_{7} & p_{2} & p_{1} & x_{6} & x_{5} & d_{2} & x_{4} & d_{1}
\end{array}
\end{aligned}
$$

The last table tells us that if we add an object $d_{1}$, to any of the naming functions in (19), we will have to add all the objects $\left(d_{1}, x_{4}, d_{2}, x_{5}, p_{1}, x_{6}, p_{2}, x_{7}\right)$ defined by

$$
\begin{array}{ll}
x_{4}=a * d_{1} & p_{1}=c_{1} * d_{1} \\
d_{2}=b * d_{1} & x_{6}=a * p_{1} \\
x_{5}=a * d_{2} & p_{2}=b * p_{1} \\
& x_{7}=a * p_{2}
\end{array}
$$


How do we choose $d_{1}$ ? If we had any more objects that commute with $a$, those would be the candidates to $d_{1}$. However, in each of our naming functions, there are no more objects that commute with $a$. The next largest value we can place in $d_{1} * a$ is $d_{2}$. We see that only some of our naming functions will satisfy this. For example, the naming function with $a=g_{1}, b=g_{3}$ is disqualified from being a canonical naming function. We can not find a new $d_{1} \notin D_{8}$ such that $d_{1} * a=b * d_{1}$. The only cases when we can find this $d_{1}$ is if we have $a, b \in\left\{g_{1}, g_{2}, g_{4}\right\}$. The easiest way to find the candidates for $d_{1}$, is to compare the row of $a$ and the column of $b$. If the $i$-th object in the row of $a$ coincides with the $i$-th object in the column of $b$, then the $i$-th object on the first column (or first row) is a candidate for $d_{1}$. For example, with the naming function $\left(g_{1}, g_{2}, g_{4}, g_{3}, g_{5}, h_{5}, h_{6}\right)$, the candidates for $d_{1}$ are the objects $g_{6}, g_{7}, h_{1}, h_{2}, h_{9}, h_{10}, h_{13}, h_{14}$. The candidates for $d_{1}$ are determined by $a, b$. If $a=g_{1}$ and $b=g_{3}$ we have no candidate for $d_{1}$. If $a=g_{1}$ and $b=g_{4}$ the candidates for $d_{1}$ are $g_{8}, g_{9}, h_{3}, h_{4}, h_{7}, h_{8}, h_{11}, h_{12}$, etc. The naming functions that satisfy this condition are those that have $a, b$ in $g_{1}, g_{2}, g_{4}$. Now we know more about the canonical naming function. We have $K(4)=\left\{e, g_{1}, g_{2}, g_{4}\right\}$ as the first four objects of the naming function. Then we have to choose a second order object $c_{1}$ that commutes with $a$. Then we choose $d_{1}$ so that $b * d_{1}=d_{1} * a$. Below we give twelve naming functions. Each of these has eight possible candidates for $d_{1}$. This means now we have a total of ninety-six possible naming functions.

$$
\begin{array}{lll}
\left(g_{1}, g_{2}, g_{4}, g_{3}, g_{5}, h_{5}, h_{6}, d_{1}, \ldots, x_{7}\right) & \left(g_{2}, g_{1}, g_{4}, g_{8}, g_{9}, h_{3}, h_{4}, d_{1}, \ldots, x_{7}\right) & \left(g_{4}, g_{1}, g_{2}, g_{6}, g_{7}, h_{1}, h_{2}, d_{1}, \ldots, x_{7}\right) \\
\left(g_{1}, g_{2}, g_{4}, g_{5}, g_{3}, h_{6}, h_{5}, d_{1}, \ldots, x_{7}\right) & \left(g_{2}, g_{1}, g_{4}, g_{9}, g_{8}, h_{4}, h_{3}, d_{1}, \ldots, x_{7}\right) & \left(g_{4}, g_{1}, g_{2}, g_{7}, g_{6}, h_{2}, h_{1}, d_{1}, \ldots, x_{7}\right) \\
\left(g_{1}, g_{4}, g_{2}, g_{3}, g_{5}, h_{6}, h_{5}, d_{1}, \ldots, x_{7}\right) & \left(g_{2}, g_{4}, g_{1}, g_{8}, g_{9}, h_{4}, h_{3}, d_{1}, \ldots, x_{7}\right) & \left(g_{4}, g_{2}, g_{1}, g_{6}, g_{7}, h_{2}, h_{1}, d_{1}, \ldots, x_{7}\right) \\
\left(g_{1}, g_{4}, g_{2}, g_{5}, g_{3}, h_{5}, h_{6}, d_{1}, \ldots, x_{7}\right) & \left(g_{2}, g_{4}, g_{1}, g_{9}, g_{8}, h_{3}, h_{4}, d_{1}, \ldots, x_{7}\right) & \left(g_{4}, g_{2}, g_{1}, g_{7}, g_{6}, h_{1}, h_{2}, d_{1}, \ldots, x_{7}\right)
\end{array}
$$

\begin{tabular}{|c|c|c|c|c|c|c|c|c|c|c|c|c|}
\hline$e \quad a$ & $b$ & $x_{1}$ & $c_{1}$ & $x_{2}$ & $c_{2}$ & $x_{3} \quad d_{1}$ & $x_{4}$ & $d_{2}$ & $x_{5}$ & $p_{1}$ & $x_{6}$ & $p_{2}$ \\
\hline$e$ & $x_{1}$ & $b$ & $x_{2}$ & $c_{1}$ & $x_{3}$ & $c_{2} \quad d_{2}$ & $x_{5}$ & $d_{1}$ & $x_{4}$ & $p_{2}$ & $x_{7}$ & $p_{1}$ \\
\hline$b \quad x_{1}$ & $e$ & $a$ & $x_{3}$ & $c_{2}$ & $x_{2}$ & $c_{1}$ & & & & & & \\
\hline$x_{1}$ & $a$ & $e$ & $c_{2}$ & $x_{3}$ & $c_{1}$ & $x_{2}$ & & & & & & \\
\hline$c_{1} \quad x_{2}$ & $c_{2}$ & $x_{3}$ & $e$ & $a$ & $b$ & $x_{1}$ & & & & & & \\
\hline$x_{2}$ & $x_{3}$ & $c_{2}$ & $a$ & $e$ & $x_{1}$ & $b$ & & & & & & \\
\hline$c_{2}$ & $c_{1}$ & $x_{2}$ & $x_{1}$ & $b$ & $a$ & $e$ & & & & & & \\
\hline$x_{3}$ & $x_{2}$ & $c_{1}$ & $b$ & $x_{1}$ & $e$ & $a$ & & & & & & \\
\hline$d_{1} \quad x_{4}$ & $d_{2}$ & $x_{5}$ & $p_{1}$ & $x_{6}$ & $x_{7}$ & $p_{2}$ & & & & & & \\
\hline$x_{4}$ & $x_{5}$ & $d_{2}$ & $x_{6}$ & $p_{1}$ & $p_{2}$ & $x_{7}$ & & & & & & \\
\hline$d_{2}$ & $d_{1}$ & $x_{4}$ & $p_{2}$ & $x_{7}$ & $x_{6}$ & $p_{1}$ & & & & & & \\
\hline$x_{5}$ & $x_{4}$ & $d_{1}$ & $x_{7}$ & $p_{2}$ & $p_{1}$ & $x_{6}$ & & & & & & \\
\hline$p_{1}$ & $x_{7}$ & $p_{2}$ & $d_{1}$ & $x_{4}$ & $d_{2}$ & $x_{5}$ & & & & & & \\
\hline $\begin{array}{ll}x_{6} & p_{1}\end{array}$ & $p_{2}$ & $x_{7}$ & $x_{4}$ & $d_{1}$ & $x_{5}$ & $d_{2}$ & & & & & & \\
\hline$p_{2} \quad x_{7}$ & $x_{6}$ & $p_{1}$ & $d_{2}$ & $x_{5}$ & $d_{1}$ & $x_{4}$ & & & & & & \\
\hline$p_{2}$ & $p_{1}$ & $x_{6}$ & $x_{5}$ & $d_{2}$ & $x_{4}$ & $d_{1}$ & & & & & & \\
\hline
\end{tabular}

Let us reduce the possible choices, further. The table given by the ninety-six candidate naming functions is

Notice some of our candidate naming functions give us $d_{1} * b=a * d_{1}$, which maximizes the representation. We will keep the naming functions that give us $b * d_{1}=d_{1} * a$ and $d_{1} * b=a * d_{1}$ simultaneously. In the case of $a=g_{1}, b=g_{2}$ the candidates for $d_{1}$ are reduced to $g_{6}, g_{7}, h_{1}, h_{2}$. The naming functions $\left(a, b, x_{1}, c_{1}, x_{2}, c_{2}, x_{3}, d_{1}, x_{4}, d_{2}, x_{5}\right)$ we now have are given below, without the components $\left(p_{1}, x_{6}, p_{2}, x_{7}\right)$. 
$\left(g_{1}, g_{2}, g_{4}, g_{3}, g_{5}, h_{5}, h_{6}, g_{6}, h_{1}, h_{2}, g_{7}, h_{7}, h_{11}, h_{8}, h_{12}\right)$ $\left(g_{1}, g_{2}, g_{4}, g_{3}, g_{5}, h_{5}, h_{6}, g_{7}, h_{2}, h_{1}, g_{6}, h_{8}, h_{12}, h_{7}, h_{11}\right)$ $\left(g_{1}, g_{2}, g_{4}, g_{3}, g_{5}, h_{5}, h_{6}, h_{1}, g_{6}, g_{7}, h_{2}, h_{11}, h_{7}, h_{8}, h_{12}\right)$ $\left(g_{1}, g_{2}, g_{4}, g_{3}, g_{5}, h_{5}, h_{6}, h_{2}, g_{7}, g_{6}, h_{1}, h_{12}, h_{8}, h_{7}, h_{11}\right)$

$\left(g_{1}, g_{2}, g_{4}, g_{5}, g_{3}, h_{6}, h_{5}, g_{6}, h_{1}, h_{2}, g_{7}, h_{11}, h_{7}, h_{12}, h_{8}\right)$ $\left(g_{1}, g_{2}, g_{4}, g_{5}, g_{3}, h_{6}, h_{5}, g_{7}, h_{2}, h_{1}, g_{6}, h_{12}, h_{8}, h_{7}, h_{11}\right)$ $\left(g_{1}, g_{2}, g_{4}, g_{5}, g_{3}, h_{6}, h_{5}, h_{1}, g_{6}, g_{7}, h_{2}, h_{7}, h_{11}, h_{8}, h_{12}\right)$ $\left(g_{1}, g_{2}, g_{4}, g_{5}, g_{3}, h_{6}, h_{5}, h_{2}, g_{7}, g_{6}, h_{1}, h_{8}, h_{12}, h_{7}, h_{11}\right)$

$\left(g_{1}, g_{4}, g_{2}, g_{3}, g_{5}, h_{5}, h_{6}, g_{8}, h_{3}, h_{4}, g_{9}, h_{9}, h_{13}, h_{10}, h_{14}\right)$ $\left(g_{1}, g_{4}, g_{2}, g_{3}, g_{5}, h_{5}, h_{6}, g_{9}, h_{4}, h_{3}, g_{8}, h_{10}, h_{14}, h_{9}, h_{13}\right)$ $\left(g_{1}, g_{4}, g_{2}, g_{3}, g_{5}, h_{5}, h_{6}, h_{3}, g_{8}, g_{9}, h_{4}, h_{13}, h_{9}, h_{14}, h_{10}\right)$ $\left(g_{1}, g_{4}, g_{2}, g_{3}, g_{5}, h_{5}, h_{6}, h_{4}, g_{9}, g_{8}, h_{3}, h_{14}, h_{10}, h_{9}, h_{13}\right)$

$\left(g_{1}, g_{4}, g_{2}, g_{5}, g_{3}, h_{6}, h_{5}, g_{8}, h_{3}, h_{4}, g_{9}, h_{13}, h_{9}, h_{14}, h_{10}\right)$ $\left(g_{1}, g_{4}, g_{2}, g_{5}, g_{3}, h_{6}, h_{5}, g_{9}, h_{4}, h_{3}, g_{8}, h_{14}, h_{10}, h_{13}, h_{9}\right)$ $\left(g_{1}, g_{4}, g_{2}, g_{5}, g_{3}, h_{6}, h_{5}, h_{3}, g_{8}, g_{9}, h_{4}, h_{9}, h_{13}, h_{10}, h_{14}\right)$ $\left(g_{1}, g_{4}, g_{2}, g_{5}, g_{3}, h_{6}, h_{5}, h_{4}, g_{9}, g_{8}, h_{3}, h_{10}, h_{14}, h_{9}, h_{13}\right)$ $\left(g_{2}, g_{1}, g_{4}, g_{8}, g_{9}, h_{3}, h_{4}, g_{6}, h_{2}, h_{1}, g_{7}, h_{13}, h_{10}, h_{9}, h_{14}\right)$ $\left(g_{2}, g_{1}, g_{4}, g_{8}, g_{9}, h_{3}, h_{4}, g_{7}, h_{1}, h_{2}, g_{6}, h_{9}, h_{14}, h_{13}, h_{10}\right)$ $\left(g_{2}, g_{1}, g_{4}, g_{8}, g_{9}, h_{3}, h_{4}, h_{1}, g_{7}, g_{6}, h_{2}, h_{14}, h_{9}, h_{13}, h_{10}\right)$ $\left(g_{2}, g_{1}, g_{4}, g_{8}, g_{9}, h_{3}, h_{4}, h_{2}, g_{6}, g_{7}, h_{1}, h_{10}, h_{13}, h_{14}, h_{9}\right)$

$\left(g_{2}, g_{1}, g_{4}, g_{9}, g_{8}, h_{4}, h_{3}, g_{6}, h_{2}, h_{1}, g_{7}, h_{10}, h_{13}, h_{14}, h_{9}\right)$ $\left(g_{2}, g_{1}, g_{4}, g_{9}, g_{8}, h_{4}, h_{3}, g_{7}, h_{1}, h_{2}, g_{6}, h_{14}, h_{9}, h_{10}, h_{13}\right)$ $\left(g_{2}, g_{1}, g_{4}, g_{9}, g_{8}, h_{4}, h_{3}, h_{1}, g_{7}, g_{6}, h_{2}, h_{9}, h_{14}, h_{13}, h_{10}\right)$ $\left(g_{2}, g_{1}, g_{4}, g_{9}, g_{8}, h_{4}, h_{3}, h_{2}, g_{6}, g_{7}, h_{1}, h_{13}, h_{10}, h_{9}, h_{14}\right)$

$\left(g_{2}, g_{4}, g_{1}, g_{8}, g_{9}, h_{3}, h_{4}, g_{3}, h_{5}, h_{6}, g_{5}, h_{8}, h_{7}, h_{11}, h_{12}\right)$ $\left(g_{2}, g_{4}, g_{1}, g_{8}, g_{9}, h_{3}, h_{4}, g_{5}, h_{6}, h_{5}, g_{3}, h_{11}, h_{12}, h_{8}, h_{7}\right)$ $\left(g_{2}, g_{4}, g_{1}, g_{8}, g_{9}, h_{3}, h_{4}, h_{5}, g_{3}, g_{5}, h_{6}, h_{7}, h_{8}, h_{12}, h_{11}\right)$ $\left(g_{2}, g_{4}, g_{1}, g_{8}, g_{9}, h_{3}, h_{4}, h_{6}, g_{5}, g_{3}, h_{5}, h_{12}, h_{11}, h_{7}, h_{8}\right)$

$\left(g_{2}, g_{4}, g_{1}, g_{9}, g_{8}, h_{4}, h_{3}, g_{3}, h_{5}, h_{6}, g_{5}, h_{7}, h_{8}, h_{12}, h_{11}\right)$ $\left(g_{2}, g_{4}, g_{1}, g_{9}, g_{8}, h_{4}, h_{3}, g_{5}, h_{6}, h_{5}, g_{3}, h_{12}, h_{11}, h_{7}, h_{8}\right)$ $\left(g_{2}, g_{4}, g_{1}, g_{9}, g_{8}, h_{4}, h_{3}, h_{5}, g_{3}, g_{5}, h_{6}, h_{8}, h_{7}, h_{11}, h_{12}\right)$ $\left(g_{2}, g_{4}, g_{1}, g_{9}, g_{8}, h_{4}, h_{3}, h_{6}, g_{5}, g_{3}, h_{5}, h_{11}, h_{12}, h_{8}, h_{7}\right)$

$$
\begin{aligned}
& \left(g_{4}, g_{1}, g_{2}, g_{6}, g_{7}, h_{1}, h_{2}, g_{8}, h_{4}, h_{3}, g_{9}, h_{11}, h_{8}, h_{7}, h_{12}\right) \\
& \left(g_{4}, g_{1}, g_{2}, g_{6}, g_{7}, h_{1}, h_{2}, g_{9}, h_{3}, h_{4}, g_{8}, h_{7}, h_{12}, h_{11}, h_{8}\right) \\
& \left(g_{4}, g_{1}, g_{2}, g_{6}, g_{7}, h_{1}, h_{2}, h_{3}, g_{9}, g_{8}, h_{4}, h_{12}, h_{7}, h_{8}, h_{11}\right) \\
& \left(g_{4}, g_{1}, g_{2}, g_{6}, g_{7}, h_{1}, h_{2}, h_{4}, g_{8}, g_{9}, h_{3}, h_{8}, h_{11}, h_{12}, h_{7}\right) \\
& \left(g_{4}, g_{1}, g_{2}, g_{7}, g_{6}, h_{2}, h_{1}, g_{8}, h_{4}, h_{3}, g_{9}, h_{8}, h_{11}, h_{12}, h_{7}\right) \\
& \left(g_{4}, g_{1}, g_{2}, g_{7}, g_{6}, h_{2}, h_{1}, g_{9}, h_{3}, h_{4}, g_{8}, h_{12}, h_{7}, h_{8}, h_{11}\right) \\
& \left(g_{4}, g_{1}, g_{2}, g_{7}, g_{6}, h_{2}, h_{1}, h_{3}, g_{9}, g_{8}, h_{4}, h_{7}, h_{12}, h_{11}, h_{8}\right) \\
& \left(g_{4}, g_{1}, g_{2}, g_{7}, g_{6}, h_{2}, h_{1}, h_{4}, g_{8}, g_{9}, h_{3}, h_{11}, h_{8}, h_{7}, h_{12}\right) \\
& \left(g_{4}, g_{2}, g_{1}, g_{6}, g_{7}, h_{1}, h_{2}, g_{3}, h_{6}, h_{5}, g_{5}, h_{10}, h_{9}, h_{13}, h_{14}\right) \\
& \left(g_{4}, g_{2}, g_{1}, g_{6}, g_{7}, h_{1}, h_{2}, g_{5}, h_{5}, h_{6}, g_{3}, h_{13}, h_{14}, h_{10}, h_{9}\right) \\
& \left(g_{4}, g_{2}, g_{1}, g_{6}, g_{7}, h_{1}, h_{2}, h_{5}, g_{5}, g_{3}, h_{6}, h_{14}, h_{13}, h_{9}, h_{10}\right) \\
& \left(g_{4}, g_{2}, g_{1}, g_{6}, g_{7}, h_{1}, h_{2}, h_{6}, g_{3}, g_{5}, h_{5}, h_{9}, h_{10}, h_{14}, h_{13}\right) \\
& \left(g_{4}, g_{2}, g_{1}, g_{7}, g_{6}, h_{2}, h_{1}, g_{3}, h_{6}, h_{5}, g_{5}, h_{9}, h_{10}, h_{14}, h_{13}\right) \\
& \left(g_{4}, g_{2}, g_{1}, g_{7}, g_{6}, h_{2}, h_{1}, g_{5}, h_{5}, h_{6}, g_{3}, h_{14}, h_{13}, h_{9}, h_{10}\right) \\
& \left(g_{4}, g_{2}, g_{1}, g_{7}, g_{6}, h_{2}, h_{1}, h_{5}, g_{5}, g_{3}, h_{6}, h_{13}, h_{14}, h_{10}, h_{9}\right) \\
& \left(g_{4}, g_{2}, g_{1}, g_{7}, g_{6}, h_{2}, h_{1}, h_{6}, g_{3}, g_{5}, h_{5}, h_{10}, h_{9}, h_{13}, h_{14}\right)
\end{aligned}
$$

The careful reader will start to notice what objects might turn out to be equivalent objects. For example, it is quite clear $g_{1}, g_{2}, g_{4}$ might probably be equivalent, but also $g_{3}, g_{5}$ and $g_{6}, g_{7}$ and $h_{1}, h_{2}$, etc. We shall see how we can reduce our possible choices. The table we have up to this point is 


$\begin{array}{llllllllllllllll}e & a & b & x_{1} & c_{1} & x_{2} & c_{2} & x_{3} & d_{1} & x_{4} & d_{2} & x_{5} & p_{1} & x_{6} & p_{2} & x_{7} \\ a & e & x_{1} & b & x_{2} & c_{1} & x_{3} & c_{2} & d_{2} & x_{5} & d_{1} & x_{4} & p_{2} & x_{7} & p_{1} & x_{6} \\ b & x_{1} & e & a & x_{3} & c_{2} & x_{2} & c_{1} & x_{4} & d_{1} & x_{5} & d_{2} & x_{6} & p_{1} & x_{7} & p_{2} \\ x_{1} & b & a & e & c_{2} & x_{3} & c_{1} & x_{2} & x_{5} & d_{2} & x_{4} & d_{1} & x_{7} & p_{2} & x_{6} & p_{1} \\ c_{1} & x_{2} & c_{2} & x_{3} & e & a & b & x_{1} & & & & & & & & \\ x_{2} & c_{1} & x_{3} & c_{2} & a & e & x_{1} & b & & & & & & & & \\ c_{2} & x_{3} & c_{1} & x_{2} & x_{1} & b & a & e & & & & & & & \\ x_{3} & c_{2} & x_{2} & c_{1} & b & x_{1} & e & a & & & & & & & \\ d_{1} & x_{4} & d_{2} & x_{5} & p_{1} & x_{6} & x_{7} & p_{2} & & & & & & & \\ x_{4} & d_{1} & x_{5} & d_{2} & x_{6} & p_{1} & p_{2} & x_{7} & & & & & & & \\ d_{2} & x_{5} & d_{1} & x_{4} & p_{2} & x_{7} & x_{6} & p_{1} & & & & & & & \\ x_{5} & d_{2} & x_{4} & d_{1} & x_{7} & p_{2} & p_{1} & x_{6} & & & & & & & \\ p_{1} & x_{6} & x_{7} & p_{2} & d_{1} & x_{4} & d_{2} & x_{5} & & & & & & & & \\ x_{6} & p_{1} & p_{2} & x_{7} & x_{4} & d_{1} & x_{5} & d_{2} & & & & & & & & \\ p_{2} & x_{7} & x_{6} & p_{1} & d_{2} & x_{5} & d_{1} & x_{4} & & & & & & & & \\ x_{7} & p_{2} & p_{1} & x_{6} & x_{5} & d_{2} & x_{4} & d_{1} & & & & & & & & \\ & & & & & & & & & & & & & \end{array}$

The naming functions of (21) all give a new object $d_{1} * c_{1}$. For example, in the naming function $\left(g_{1}, g_{2}, g_{4}, g_{3}, g_{5}, h_{5}, h_{6}, g_{6}, h_{1}, h_{2}, g_{7}\right)$, we have $d_{1} * c_{1}=g_{6} * g_{3}=h_{10}$ which is an object not yet named. Including this new object, $q_{1}=d_{1} * c_{1}$, gives the table

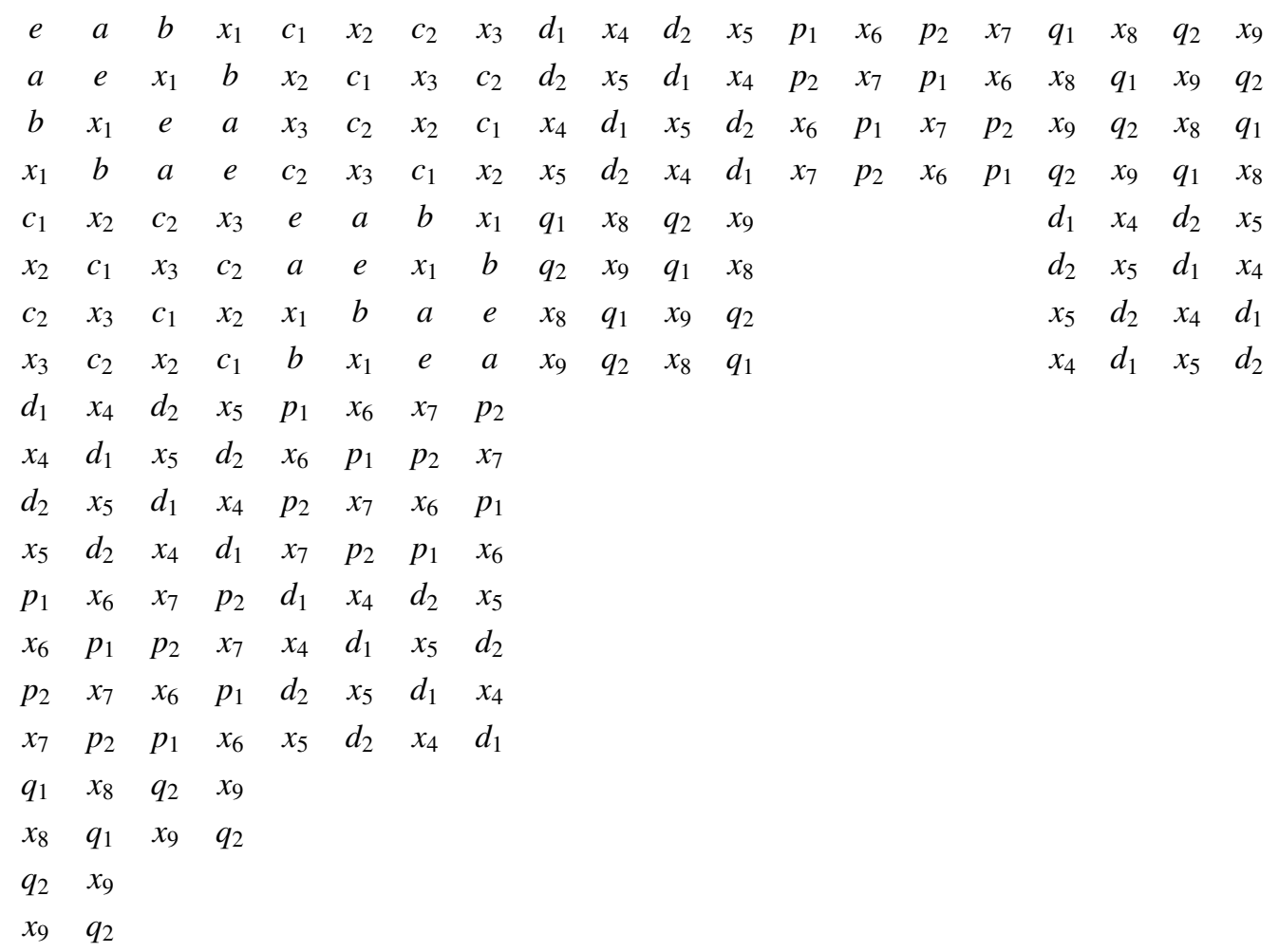

This last table tells us that if we want to maximize the representation, we will have to consider the naming functions that have $\left|d_{1}\right|=2$. These are 
$\left(g_{1}, g_{2}, g_{4}, g_{3}, g_{5}, h_{5}, h_{6}, g_{6}, h_{1}, h_{2}, g_{7}, h_{7}, h_{11}, h_{8}, h_{12}, h_{10}, h_{14}, h_{13}, h_{9}\right)$ $\left(g_{1}, g_{2}, g_{4}, g_{3}, g_{5}, h_{5}, h_{6}, g_{7}, h_{2}, h_{1}, g_{6}, h_{8}, h_{12}, h_{7}, h_{11}, h_{9}, h_{13}, h_{14}, h_{10}\right)$ $\left(g_{1}, g_{2}, g_{4}, g_{5}, g_{3}, h_{6}, h_{5}, g_{6}, h_{1}, h_{2}, g_{7}, h_{11}, h_{7}, h_{12}, h_{8}, h_{13}, h_{9}, h_{10}, h_{14}\right)$ $\left(g_{1}, g_{2}, g_{4}, g_{5}, g_{3}, h_{6}, h_{5}, g_{7}, h_{2}, h_{1}, g_{6}, h_{12}, h_{8}, h_{7}, h_{11}, h_{14}, h_{10}, h_{13}, h_{9}\right)$

$\left(g_{2}, g_{1}, g_{4}, g_{8}, g_{9}, h_{3}, h_{4}, g_{6}, h_{2}, h_{1}, g_{7}, h_{13}, h_{10}, h_{9}, h_{14}, h_{11}, h_{12}, h_{7}, h_{8}\right)$ $\left(g_{2}, g_{1}, g_{4}, g_{8}, g_{9}, h_{3}, h_{4}, g_{7}, h_{1}, h_{2}, g_{6}, h_{9}, h_{14}, h_{13}, h_{10}, h_{8}, h_{7}, h_{12}, h_{11}\right)$ $\left(g_{2}, g_{1}, g_{4}, g_{9}, g_{8}, h_{4}, h_{3}, g_{6}, h_{2}, h_{1}, g_{7}, h_{10}, h_{13}, h_{14}, h_{9}, h_{7}, h_{8}, h_{11}, h_{12}\right)$ $\left(g_{2}, g_{1}, g_{4}, g_{9}, g_{8}, h_{4}, h_{3}, g_{7}, h_{1}, h_{2}, g_{6}, h_{14}, h_{9}, h_{10}, h_{13}, h_{12}, h_{11}, h_{8}, h_{7}\right)$

$\left(g_{4}, g_{1}, g_{2}, g_{6}, g_{7}, h_{1}, h_{2}, g_{8}, h_{4}, h_{3}, g_{9}, h_{11}, h_{8}, h_{7}, h_{12}, h_{13}, h_{14}, h_{9}, h_{10}\right)$ $\left(g_{4}, g_{1}, g_{2}, g_{6}, g_{7}, h_{1}, h_{2}, g_{9}, h_{3}, h_{4}, g_{8}, h_{7}, h_{12}, h_{11}, h_{8}, h_{10}, h_{9}, h_{14}, h_{13}\right)$ $\left(g_{4}, g_{1}, g_{2}, g_{7}, g_{6}, h_{2}, h_{1}, g_{8}, h_{4}, h_{3}, g_{9}, h_{8}, h_{11}, h_{12}, h_{7}, h_{9}, h_{10}, h_{13}, h_{14}\right)$ $\left(g_{4}, g_{1}, g_{2}, g_{7}, g_{6}, h_{2}, h_{1}, g_{9}, h_{3}, h_{4}, g_{8}, h_{12}, h_{7}, h_{8}, h_{11}, h_{14}, h_{13}, h_{10}, h_{9}\right)$ $\left(g_{1}, g_{4}, g_{2}, g_{3}, g_{5}, h_{5}, h_{6}, g_{8}, h_{3}, h_{4}, g_{9}, h_{9}, h_{13}, h_{10}, h_{14}, h_{8}, h_{12}, h_{11}, h_{7}\right)$ $\left(g_{1}, g_{4}, g_{2}, g_{3}, g_{5}, h_{5}, h_{6}, g_{9}, h_{4}, h_{3}, g_{8}, h_{10}, h_{14}, h_{9}, h_{13}, h_{7}, h_{11}, h_{12}, h_{8}\right)$ $\left(g_{1}, g_{4}, g_{2}, g_{5}, g_{3}, h_{6}, h_{5}, g_{8}, h_{3}, h_{4}, g_{9}, h_{13}, h_{9}, h_{14}, h_{10}, h_{11}, h_{7}, h_{8}, h_{12}\right)$ $\left(g_{1}, g_{4}, g_{2}, g_{5}, g_{3}, h_{6}, h_{5}, g_{9}, h_{4}, h_{3}, g_{8}, h_{14}, h_{13}, h_{10}, h_{9}, h_{12}, h_{8}, h_{7}, h_{11}\right)$

$\left(g_{2}, g_{4}, g_{1}, g_{8}, g_{9}, h_{3}, h_{4}, g_{3}, h_{5}, h_{6}, g_{5}, h_{8}, h_{7}, h_{11}, h_{12}, h_{9}, h_{14}, h_{10}, h_{13}\right)$ $\left(g_{2}, g_{4}, g_{1}, g_{8}, g_{9}, h_{3}, h_{4}, g_{5}, h_{6}, h_{5}, g_{3}, h_{11}, h_{12}, h_{8}, h_{7}, h_{13}, h_{10}, h_{14}, h_{9}\right)$ $\left(g_{2}, g_{4}, g_{1}, g_{9}, g_{8}, h_{4}, h_{3}, g_{3}, h_{5}, h_{6}, g_{5}, h_{7}, h_{8}, h_{12}, h_{11}, h_{10}, h_{13}, h_{9}, h_{14}\right)$ $\left(g_{2}, g_{4}, g_{1}, g_{9}, g_{8}, h_{4}, h_{3}, g_{5}, h_{6}, h_{5}, g_{3}, h_{12}, h_{11}, h_{7}, h_{8}, h_{14}, h_{9}, h_{13}, h_{10}\right)$

$\left(g_{4}, g_{2}, g_{1}, g_{6}, g_{7}, h_{1}, h_{2}, g_{3}, h_{6}, h_{5}, g_{5}, h_{10}, h_{9}, h_{13}, h_{14}, h_{7}, h_{12}, h_{8}, h_{11}\right)$ $\left(g_{4}, g_{2}, g_{1}, g_{6}, g_{7}, h_{1}, h_{2}, g_{5}, h_{5}, h_{6}, g_{3}, h_{13}, h_{14}, h_{10}, h_{9}, h_{11}, h_{8}, h_{7}, h_{12}\right)$ $\left(g_{4}, g_{2}, g_{1}, g_{7}, g_{6}, h_{2}, h_{1}, g_{3}, h_{6}, h_{5}, g_{5}, h_{9}, h_{10}, h_{14}, h_{13}, h_{8}, h_{11}, h_{7}, h_{14}\right)$ $\left(g_{4}, g_{2}, g_{1}, g_{7}, g_{6}, h_{2}, h_{1}, g_{5}, h_{5}, h_{6}, g_{3}, h_{14}, h_{13}, h_{9}, h_{10}, h_{12}, h_{7}, h_{11}, h_{8}\right)$

These naming functions give the table

\begin{tabular}{|c|c|c|c|c|c|c|c|c|c|c|c|c|c|c|c|c|c|c|c|}
\hline$e$ & $a$ & $b$ & $x_{1}$ & $c_{1}$ & $x_{2}$ & $c_{2}$ & $x_{3}$ & $d_{1}$ & $x_{4}$ & $d_{2}$ & $x_{5}$ & $p_{1}$ & $x_{6}$ & $p_{2}$ & $x_{7}$ & $q_{1}$ & $x_{8}$ & $q_{2}$ & $x_{9}$ \\
\hline$a$ & $e$ & $x_{1}$ & $b$ & $x_{2}$ & $c_{1}$ & $x_{3}$ & $c_{2}$ & $d_{2}$ & $x_{5}$ & $d_{1}$ & $x_{4}$ & $p_{2}$ & $x_{7}$ & $p_{1}$ & $x_{6}$ & $x_{8}$ & $q_{1}$ & $x_{9}$ & $q_{2}$ \\
\hline$b$ & $x_{1}$ & $e$ & $a$ & $x_{3}$ & $c_{2}$ & $x_{2}$ & $c_{1}$ & $x_{4}$ & $d_{1}$ & $x_{5}$ & $d_{2}$ & $x_{6}$ & $p_{1}$ & $x_{7}$ & $p_{2}$ & $x_{9}$ & $q_{2}$ & $x_{8}$ & $q_{1}$ \\
\hline$x_{1}$ & $b$ & $a$ & $e$ & $c_{2}$ & $x_{3}$ & $c_{1}$ & $x_{2}$ & $x_{5}$ & $d_{2}$ & $x_{4}$ & $d_{1}$ & $x_{7}$ & $p_{2}$ & $x_{6}$ & $p_{1}$ & $q_{2}$ & $x_{9}$ & $q_{1}$ & $x_{8}$ \\
\hline$c_{1}$ & $x_{2}$ & $c_{2}$ & $x_{3}$ & $e$ & $a$ & $b$ & $x_{1}$ & $q_{1}$ & $x_{8}$ & $q_{2}$ & $x_{9}$ & & & & & $d_{1}$ & $x_{4}$ & $d_{2}$ & $x_{5}$ \\
\hline$x_{2}$ & $c_{1}$ & $x_{3}$ & $c_{2}$ & $a$ & $e$ & $x_{1}$ & $b$ & $q_{2}$ & $x_{9}$ & $q_{1}$ & $x_{8}$ & & & & & $d_{2}$ & $x_{5}$ & $d_{1}$ & $x_{4}$ \\
\hline$c_{2}$ & $x_{3}$ & $c_{1}$ & $x_{2}$ & $x_{1}$ & $b$ & $a$ & $e$ & $x_{8}$ & $q_{1}$ & $x_{9}$ & $q_{2}$ & & & & & $x_{5}$ & $d_{2}$ & $x_{4}$ & $d_{1}$ \\
\hline$x_{3}$ & $c_{2}$ & $x_{2}$ & $c_{1}$ & $b$ & $x_{1}$ & $e$ & $a$ & $x_{9}$ & $q_{2}$ & $x_{8}$ & $q_{1}$ & & & & & $x_{4}$ & $d_{1}$ & $x_{5}$ & $d_{2}$ \\
\hline$d_{1}$ & $x_{4}$ & $d_{2}$ & $x_{5}$ & $p_{1}$ & $x_{6}$ & $x_{7}$ & $p_{2}$ & $e$ & $a$ & $b$ & $x_{1}$ & $c_{1}$ & $x_{2}$ & $x_{3}$ & $c_{2}$ & & & & \\
\hline$x_{4}$ & $d_{1}$ & $x_{5}$ & $d_{2}$ & $x_{6}$ & $p_{1}$ & $p_{2}$ & $x_{7}$ & $b$ & $x_{1}$ & $e$ & $a$ & $x_{3}$ & $c_{2}$ & $c_{1}$ & $x_{2}$ & & & & \\
\hline$d_{2}$ & $x_{5}$ & $d_{1}$ & $x_{4}$ & $p_{2}$ & $x_{7}$ & $x_{6}$ & $p_{1}$ & $a$ & $e$ & $x_{1}$ & $b$ & $x_{2}$ & $c_{1}$ & $c_{2}$ & $x_{3}$ & & & & \\
\hline$x_{5}$ & $d_{2}$ & $x_{4}$ & $d_{1}$ & $x_{7}$ & $p_{2}$ & $p_{1}$ & $x_{6}$ & $x_{1}$ & $b$ & $a$ & $e$ & $c_{2}$ & $x_{3}$ & $x_{3}$ & $c_{2}$ & & & & \\
\hline$p_{1}$ & $x_{6}$ & $x_{7}$ & $p_{2}$ & $d_{1}$ & $x_{4}$ & $d_{2}$ & $x_{5}$ & & & & & & & & & $e$ & $a$ & $b$ & $x_{1}$ \\
\hline$x_{6}$ & $p_{1}$ & $p_{2}$ & $x_{7}$ & $x_{4}$ & $d_{1}$ & $x_{5}$ & $d_{2}$ & & & & & & & & & $b$ & $x_{1}$ & $e$ & $a$ \\
\hline$p_{2}$ & $x_{7}$ & $x_{6}$ & $p_{1}$ & $d_{2}$ & $x_{5}$ & $d_{1}$ & $x_{4}$ & & & & & & & & & $a$ & $e$ & $x_{1}$ & $b$ \\
\hline$x_{7}$ & $p_{2}$ & $p_{1}$ & $x_{6}$ & $x_{5}$ & $d_{2}$ & $x_{4}$ & $d_{1}$ & & & & & & & & & $x_{1}$ & $b$ & $a$ & $e$ \\
\hline$q_{1}$ & $x_{8}$ & $q_{2}$ & $x_{9}$ & & & & & $c_{1}$ & $x_{2}$ & $c_{2}$ & $x_{3}$ & $e$ & $a$ & $x_{1}$ & $b$ & & & & \\
\hline$x_{8}$ & $q_{1}$ & $x_{9}$ & $q_{2}$ & & & & & $c_{2}$ & $x_{3}$ & $c_{1}$ & $x_{2}$ & $x_{1}$ & $b$ & $e$ & $a$ & & & & \\
\hline$q_{2}$ & $x_{9}$ & $q_{1}$ & $x_{8}$ & & & & & $x_{2}$ & $c_{1}$ & $x_{3}$ & $c_{2}$ & $a$ & $e$ & $b$ & $x_{1}$ & & & & \\
\hline$x_{9}$ & $q_{2}$ & $x_{8}$ & $q_{1}$ & & & & & $x_{3}$ & $c_{2}$ & $x_{2}$ & $c_{1}$ & $b$ & $x_{1}$ & $a$ & $e$ & & & & \\
\hline
\end{tabular}

We see that we have to add a new object $r_{1}=c_{1} * q_{1}$. We finally have $x_{10}=a * r_{1}, r_{2}=b * r_{1}, x_{11}=a * r_{2}$. This completes our naming functions. 
$\left(g_{1}, g_{2}, g_{4}, g_{3}, g_{5}, h_{5}, h_{6}, g_{6}, h_{1}, h_{2}, g_{7}, h_{7}, h_{11}, h_{8}, h_{12}, h_{10}, h_{14}, h_{13}, h_{9}, g_{9}, h_{4}, g_{8}, h_{3}\right)$

$\left(g_{1}, g_{2}, g_{4}, g_{3}, g_{5}, h_{5}, h_{6}, g_{7}, h_{2}, h_{1}, g_{6}, h_{8}, h_{12}, h_{7}, h_{11}, h_{9}, h_{13}, h_{14}, h_{10}, g_{8}, h_{3}, g_{9}, h_{4}\right)$

$\left(g_{1}, g_{2}, g_{4}, g_{5}, g_{3}, h_{6}, h_{5}, g_{6}, h_{1}, h_{2}, g_{7}, h_{11}, h_{7}, h_{12}, h_{8}, h_{13}, h_{9}, h_{10}, h_{14}, g_{8}, h_{3}, g_{9}, h_{4}\right)$

$\left(g_{1}, g_{2}, g_{4}, g_{5}, g_{3}, h_{6}, h_{5}, g_{7}, h_{2}, h_{1}, g_{6}, h_{12}, h_{8}, h_{7}, h_{11}, h_{14}, h_{10}, h_{13}, h_{9}, g_{9}, h_{4}, g_{8}, h_{3}\right)$

$\left(g_{1}, g_{4}, g_{2}, g_{3}, g_{5}, h_{5}, h_{6}, g_{8}, h_{3}, h_{4}, g_{9}, h_{9}, h_{13}, h_{10}, h_{14}, h_{8}, h_{12}, h_{11}, h_{7}, g_{7}, h_{2}, g_{6}, h_{1}\right)$

$\left(g_{1}, g_{4}, g_{2}, g_{3}, g_{5}, h_{5}, h_{6}, g_{9}, h_{4}, h_{3}, g_{8}, h_{10}, h_{14}, h_{9}, h_{13}, h_{7}, h_{11}, h_{12}, h_{8}, g_{6}, h_{1}, g_{7}, h_{2}\right)$

$\left(g_{1}, g_{4}, g_{2}, g_{5}, g_{3}, h_{6}, h_{5}, g_{8}, h_{3}, h_{4}, g_{9}, h_{13}, h_{9}, h_{14}, h_{10}, h_{11}, h_{7}, h_{8}, h_{12}, g_{6}, h_{1}, g_{7}, h_{2}\right)$

$\left(g_{1}, g_{4}, g_{2}, g_{5}, g_{3}, h_{6}, h_{5}, g_{9}, h_{4}, h_{3}, g_{8}, h_{14}, h_{13}, h_{10}, h_{9}, h_{12}, h_{8}, h_{7}, h_{11}, g_{7}, h_{2}, g_{6}, h_{1}\right)$

$\left(g_{2}, g_{1}, g_{4}, g_{8}, g_{9}, h_{3}, h_{4}, g_{6}, h_{2}, h_{1}, g_{7}, h_{13}, h_{10}, h_{9}, h_{14}, h_{11}, h_{12}, h_{7}, h_{8}, g_{5}, h_{6}, g_{3}, h_{5}\right)$

$\left(g_{2}, g_{1}, g_{4}, g_{8}, g_{9}, h_{3}, h_{4}, g_{7}, h_{1}, h_{2}, g_{6}, h_{9}, h_{14}, h_{13}, h_{10}, h_{8}, h_{7}, h_{12}, h_{11}, g_{3}, h_{5}, g_{5}, h_{6}\right)$

$\left(g_{2}, g_{1}, g_{4}, g_{9}, g_{8}, h_{4}, h_{3}, g_{6}, h_{2}, h_{1}, g_{7}, h_{10}, h_{13}, h_{14}, h_{9}, h_{7}, h_{8}, h_{11}, h_{12}, g_{3}, h_{5}, g_{5}, h_{6}\right)$

$\left(g_{2}, g_{1}, g_{4}, g_{9}, g_{8}, h_{4}, h_{3}, g_{7}, h_{1}, h_{2}, g_{6}, h_{14}, h_{9}, h_{10}, h_{13}, h_{12}, h_{11}, h_{8}, h_{7}, g_{5}, h_{6}, g_{3}, h_{5}\right)$

$\left(g_{2}, g_{4}, g_{1}, g_{8}, g_{9}, h_{3}, h_{4}, g_{3}, h_{5}, h_{6}, g_{5}, h_{8}, h_{7}, h_{11}, h_{12}, h_{9}, h_{14}, h_{10}, h_{13}, g_{7}, h_{1}, g_{6}, h_{2}\right)$

$\left(g_{2}, g_{4}, g_{1}, g_{8}, g_{9}, h_{3}, h_{4}, g_{5}, h_{6}, h_{5}, g_{3}, h_{11}, h_{12}, h_{8}, h_{7}, h_{13}, h_{10}, h_{14}, h_{9}, g_{6}, h_{2}, g_{7}, h_{1}\right)$

$\left(g_{2}, g_{4}, g_{1}, g_{9}, g_{8}, h_{4}, h_{3}, g_{3}, h_{5}, h_{6}, g_{5}, h_{7}, h_{8}, h_{12}, h_{11}, h_{10}, h_{13}, h_{9}, h_{14}, g_{6}, h_{2}, g_{7}, h_{1}\right)$

$\left(g_{2}, g_{4}, g_{1}, g_{9}, g_{8}, h_{4}, h_{3}, g_{5}, h_{6}, h_{5}, g_{3}, h_{12}, h_{11}, h_{7}, h_{8}, h_{14}, h_{9}, h_{13}, h_{10}, g_{7}, h_{1}, g_{6}, h_{2}\right)$

$\left(g_{4}, g_{1}, g_{2}, g_{6}, g_{7}, h_{1}, h_{2}, g_{8}, h_{4}, h_{3}, g_{9}, h_{11}, h_{8}, h_{7}, h_{12}, h_{13}, h_{14}, h_{9}, h_{10}, g_{5}, h_{5}, g_{3}, h_{6}\right)$

$\left(g_{4}, g_{1}, g_{2}, g_{6}, g_{7}, h_{1}, h_{2}, g_{9}, h_{3}, h_{4}, g_{8}, h_{7}, h_{12}, h_{11}, h_{8}, h_{10}, h_{9}, h_{14}, h_{13}, g_{3}, h_{6}, g_{5}, h_{5}\right)$

$\left(g_{4}, g_{1}, g_{2}, g_{7}, g_{6}, h_{2}, h_{1}, g_{8}, h_{4}, h_{3}, g_{9}, h_{8}, h_{11}, h_{12}, h_{7}, h_{9}, h_{10}, h_{13}, h_{14}, g_{3}, h_{6}, g_{5}, h_{5}\right)$

$\left(g_{4}, g_{1}, g_{2}, g_{7}, g_{6}, h_{2}, h_{1}, g_{9}, h_{3}, h_{4}, g_{8}, h_{12}, h_{7}, h_{8}, h_{11}, h_{14}, h_{13}, h_{10}, h_{9}, g_{5}, h_{5}, g_{3}, h_{6}\right)$

$\left(g_{4}, g_{2}, g_{1}, g_{6}, g_{7}, h_{1}, h_{2}, g_{3}, h_{6}, h_{5}, g_{5}, h_{10}, h_{9}, h_{13}, h_{14}, h_{7}, h_{12}, h_{8}, h_{11}, g_{9}, h_{3}, g_{8}, h_{4}\right)$

$\left(g_{4}, g_{2}, g_{1}, g_{6}, g_{7}, h_{1}, h_{2}, g_{5}, h_{5}, h_{6}, g_{3}, h_{13}, h_{14}, h_{10}, h_{9}, h_{11}, h_{8}, h_{7}, h_{12}, g_{8}, h_{4}, g_{9}, h_{3}\right)$

$\left(g_{4}, g_{2}, g_{1}, g_{7}, g_{6}, h_{2}, h_{1}, g_{3}, h_{6}, h_{5}, g_{5}, h_{9}, h_{10}, h_{14}, h_{13}, h_{8}, h_{11}, h_{7}, h_{14}, g_{8}, h_{4}, g_{9}, h_{3}\right)$

$\left(g_{4}, g_{2}, g_{1}, g_{7}, g_{6}, h_{2}, h_{1}, g_{5}, h_{5}, h_{6}, g_{3}, h_{14}, h_{13}, h_{9}, h_{10}, h_{12}, h_{7}, h_{11}, h_{8}, g_{9}, h_{3}, g_{8}, h_{4}\right)$

We have found the canonical naming function of the permutation group $\Delta_{4}$. We have also been able to identify equivalent objects of the group. The equivalence classes of objects are

$$
\begin{gathered}
\{e\} \\
\left\{g_{1}, g_{2}, g_{4}\right\} \\
\left\{g_{3}, g_{5}, g_{6}, g_{7}, g_{8}, g_{9}\right\} \\
\left\{h_{1}, h_{2}, h_{3}, h_{4}, h_{5}, h_{6},\right\} \\
\left\{h_{7}, h_{8}, h_{9}, h_{10}, h_{11}, h_{12}, h_{13}, h_{14}\right\}
\end{gathered}
$$

The naming functions above give us the canonical table in block form. 


\begin{tabular}{|c|c|c|c|c|c|c|c|c|c|c|c|c|c|c|c|c|c|c|c|c|c|c|c|}
\hline$e$ & $a$ & $b$ & $x_{1}$ & $c_{1}$ & $x_{2}$ & $c_{2}$ & $x_{3}$ & $d_{1}$ & $x_{4}$ & $d_{2}$ & $x_{5}$ & $p_{1}$ & $x_{6}$ & $p_{2}$ & $x_{7}$ & $q_{1}$ & $x_{8}$ & $q_{2}$ & $x_{9}$ & $r_{1}$ & $x_{10}$ & $r_{2}$ & $x_{11}$ \\
\hline$a$ & $e$ & $x_{1}$ & $b$ & $x_{2}$ & $c_{1}$ & $x_{3}$ & $c_{2}$ & $d_{2}$ & $x_{5}$ & $d_{1}$ & $x_{4}$ & $p_{2}$ & $x_{7}$ & $p_{1}$ & $x_{6}$ & $x_{8}$ & $q_{1}$ & $x_{9}$ & $q_{2}$ & $x_{10}$ & $r_{1}$ & $x_{11}$ & $r_{2}$ \\
\hline$b$ & $x_{1}$ & $e$ & $a$ & $x_{3}$ & $c_{2}$ & $x_{2}$ & $c_{1}$ & $x_{4}$ & $d_{1}$ & $x_{5}$ & $d_{2}$ & $x_{6}$ & $p_{1}$ & $x_{7}$ & $p_{2}$ & $x_{9}$ & $q_{2}$ & $x_{8}$ & $q_{1}$ & $r_{2}$ & $x_{11}$ & $r_{1}$ & $x_{10}$ \\
\hline$x_{1}$ & $b$ & $a$ & $e$ & $c_{2}$ & $x_{3}$ & $c_{1}$ & $x_{2}$ & $x_{5}$ & $d_{2}$ & $x_{4}$ & $d_{1}$ & $x_{7}$ & $p_{2}$ & $x_{6}$ & $p_{1}$ & $q_{2}$ & $x_{9}$ & $q_{1}$ & $x_{8}$ & $x_{11}$ & $r_{2}$ & $x_{10}$ & $r_{1}$ \\
\hline$c_{1}$ & $x_{2}$ & $c_{2}$ & $x_{3}$ & $e$ & $a$ & $b$ & $x_{1}$ & $q_{1}$ & $x_{8}$ & $q_{2}$ & $x_{9}$ & $r_{1}$ & $x_{10}$ & $x_{11}$ & $r_{2}$ & $d_{1}$ & $x_{4}$ & $d_{2}$ & $x_{5}$ & $p_{1}$ & $x_{6}$ & $x_{7}$ & $p_{2}$ \\
\hline$x_{2}$ & $c_{1}$ & $x_{3}$ & $c_{2}$ & $a$ & $e$ & $x_{1}$ & $b$ & $q_{2}$ & $x_{9}$ & $q_{1}$ & $x_{8}$ & $x_{11}$ & $r_{2}$ & $r_{1}$ & $x_{10}$ & $d_{2}$ & $x_{5}$ & $d_{1}$ & $x_{4}$ & $p_{2}$ & $x_{7}$ & $x_{6}$ & $p_{1}$ \\
\hline$c_{2}$ & $x_{3}$ & $c_{1}$ & $x_{2}$ & $x_{1}$ & $b$ & $a$ & $e$ & $x_{8}$ & $q_{1}$ & $x_{9}$ & $q_{2}$ & $x_{10}$ & $r_{1}$ & $r_{2}$ & $x_{11}$ & $x_{5}$ & $d_{2}$ & $x_{4}$ & $d_{1}$ & $x_{7}$ & $p_{2}$ & $p_{1}$ & $x_{6}$ \\
\hline$x_{3}$ & $c_{2}$ & $x_{2}$ & $c_{1}$ & $b$ & $x_{1}$ & $e$ & $a$ & $x_{9}$ & $q_{2}$ & $x_{8}$ & $q_{1}$ & $r_{2}$ & $x_{11}$ & $x_{10}$ & $r_{1}$ & $x_{4}$ & $d_{1}$ & $x_{5}$ & $d_{2}$ & $x_{6}$ & $p_{1}$ & $p_{2}$ & $x_{7}$ \\
\hline$d_{1}$ & $x_{4}$ & $d_{2}$ & $x_{5}$ & $p_{1}$ & $x_{6}$ & $x_{7}$ & $p_{2}$ & $e$ & $a$ & $b$ & $x_{1}$ & $c_{1}$ & $x_{2}$ & $x_{3}$ & $c_{2}$ & $r_{1}$ & $x_{10}$ & $r_{2}$ & $x_{11}$ & $q_{1}$ & $x_{8}$ & $q_{2}$ & $x_{9}$ \\
\hline$x_{4}$ & $d_{1}$ & $x_{5}$ & $d_{2}$ & $x_{6}$ & $p_{1}$ & $p_{2}$ & $x_{7}$ & $b$ & $x_{1}$ & $e$ & $a$ & $x_{3}$ & $c_{2}$ & $c_{1}$ & $x_{2}$ & $r_{2}$ & $x_{11}$ & $x_{10}$ & $r_{1}$ & $x_{9}$ & $q_{2}$ & $x_{8}$ & $q_{1}$ \\
\hline$d_{2}$ & $x_{5}$ & $d_{1}$ & $x_{4}$ & $p_{2}$ & $x_{7}$ & $x_{6}$ & $p_{1}$ & $a$ & $e$ & $x_{1}$ & $b$ & $x_{2}$ & $c_{1}$ & $c_{2}$ & $x_{3}$ & $x_{11}$ & $r_{2}$ & $r_{1}$ & $x_{10}$ & $q_{2}$ & $x_{9}$ & $q_{1}$ & 0 \\
\hline$x_{5}$ & $d_{2}$ & $x_{4}$ & $d_{1}$ & $x_{7}$ & $p_{2}$ & $p_{1}$ & $x_{6}$ & $x_{1}$ & $b$ & $a$ & $e$ & $c_{2}$ & $x_{3}$ & $x_{3}$ & $c_{2}$ & $x_{10}$ & $r_{1}$ & $x_{11}$ & $r_{2}$ & $x_{8}$ & $q_{1}$ & $x_{9}$ & $q_{2}$ \\
\hline$p_{1}$ & $x_{6}$ & $x_{7}$ & $p_{2}$ & $d_{1}$ & $x_{4}$ & $d_{2}$ & $x_{5}$ & $r_{1}$ & $x_{10}$ & $r_{2}$ & $x_{11}$ & $q_{1}$ & $x_{8}$ & $q_{2}$ & $x_{9}$ & $e$ & $a$ & $b$ & $x_{1}$ & $c_{1}$ & $x_{2}$ & $c_{2}$ & $x_{3}$ \\
\hline$x_{6}$ & $p_{1}$ & $p_{2}$ & $x_{7}$ & $x_{4}$ & $d_{1}$ & $x_{5}$ & $d_{2}$ & $r_{2}$ & $x_{11}$ & $r_{1}$ & $x_{10}$ & $x_{9}$ & $q_{2}$ & $q_{1}$ & $x_{8}$ & $b$ & $x_{1}$ & $e$ & $a$ & $x_{3}$ & $c_{2}$ & $x_{2}$ & $c_{1}$ \\
\hline$p_{2}$ & $x_{7}$ & $x_{6}$ & $p_{1}$ & $d_{2}$ & $x_{5}$ & $d_{1}$ & $x_{4}$ & $x_{11}$ & $r_{2}$ & $x_{10}$ & $r_{1}$ & $q_{2}$ & $x_{9}$ & $x_{8}$ & $q_{1}$ & $a$ & $e$ & $x_{1}$ & $b$ & $x_{2}$ & $c_{1}$ & $x_{3}$ & $c_{2}$ \\
\hline$x_{7}$ & $p_{2}$ & $p_{1}$ & $x_{6}$ & $x_{5}$ & $d_{2}$ & $x_{4}$ & $d_{1}$ & $x_{10}$ & $r_{1}$ & $x_{11}$ & $r_{2}$ & $x_{8}$ & $q_{1}$ & $x_{9}$ & $q_{2}$ & $x_{1}$ & $b$ & $a$ & e & $c_{2}$ & $x_{3}$ & $c_{1}$ & $x_{2}$ \\
\hline$q_{1}$ & $x_{8}$ & $q_{2}$ & $x_{9}$ & $r_{1}$ & $x_{10}$ & $r_{2}$ & $x_{11}$ & $c_{1}$ & $x_{2}$ & $c_{2}$ & $x_{3}$ & $e$ & $a$ & $x_{1}$ & $b$ & $p_{1}$ & $x_{6}$ & $p_{2}$ & $x_{7}$ & $d_{1}$ & $x_{4}$ & $d_{2}$ & $x_{5}$ \\
\hline$x_{8}$ & $q_{1}$ & $x_{9}$ & $q_{2}$ & $x_{10}$ & $r_{1}$ & $x_{11}$ & $r_{2}$ & $c_{2}$ & $x_{3}$ & $c_{1}$ & $x_{2}$ & $x_{1}$ & $b$ & $e$ & u & $x_{7}$ & $p_{2}$ & $p_{1}$ & $x_{6}$ & $x_{5}$ & $d_{2}$ & $x_{4}$ & $d_{1}$ \\
\hline$q_{2}$ & $x_{9}$ & $q_{1}$ & $x_{8}$ & $x_{11}$ & $r_{2}$ & $x_{10}$ & $r_{1}$ & $x_{2}$ & $c_{1}$ & $x_{3}$ & $c_{2}$ & $a$ & $e$ & $b$ & $x_{1}$ & $p_{2}$ & $x_{7}$ & $x_{6}$ & $p_{1}$ & $d_{2}$ & $x_{5}$ & $d_{1}$ & $x_{4}$ \\
\hline$x_{9}$ & $q_{2}$ & $x_{8}$ & $q_{1}$ & $r_{2}$ & $x_{11}$ & $r_{1}$ & $x_{10}$ & $x_{3}$ & $c_{2}$ & $x_{2}$ & $c_{1}$ & $b$ & $x_{1}$ & $a$ & $e$ & $x_{6}$ & $p_{1}$ & $x_{7}$ & $p_{2}$ & $x_{4}$ & $d_{1}$ & $x_{5}$ & $d_{2}$ \\
\hline$r_{1}$ & $x_{10}$ & $r_{2}$ & $x_{11}$ & $q_{1}$ & $x_{8}$ & $q_{2}$ & $x_{9}$ & $p_{1}$ & $x_{6}$ & $p_{2}$ & $x_{7}$ & $d_{1}$ & $x_{4}$ & $d_{2}$ & $x_{5}$ & $c_{1}$ & $x_{2}$ & $c_{2}$ & $x_{3}$ & $e$ & $a$ & $b$ & $x_{1}$ \\
\hline$x_{10}$ & $r_{1}$ & $x_{11}$ & $r_{2}$ & $x_{8}$ & $q_{1}$ & $x_{9}$ & $q_{2}$ & $p_{2}$ & $x_{7}$ & $x_{6}$ & $p_{1}$ & $d_{2}$ & $x_{5}$ & $d_{1}$ & $x_{4}$ & $x_{2}$ & $c_{1}$ & $x_{3}$ & $c_{2}$ & $a$ & $e$ & $x_{1}$ & 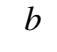 \\
\hline$r_{2}$ & $x_{11}$ & $r_{1}$ & $x_{10}$ & $x_{9}$ & $q_{2}$ & $x_{8}$ & $q_{1}$ & $x_{6}$ & $p_{1}$ & $x_{7}$ & $p_{2}$ & $x_{4}$ & $d_{1}$ & $x_{5}$ & $d_{2}$ & $x_{3}$ & $c_{2}$ & $x_{2}$ & $c_{1}$ & $b$ & $x_{1}$ & $e$ & $a$ \\
\hline$x_{11}$ & $r_{2}$ & $x_{10}$ & $r_{1}$ & $q_{2}$ & $x_{9}$ & $q_{1}$ & $x_{8}$ & $x_{7}$ & $p_{2}$ & $p_{1}$ & $x_{6}$ & $x_{5}$ & $d_{2}$ & $x_{4}$ & $d_{1}$ & $c_{2}$ & $x_{3}$ & $c_{1}$ & $x_{2}$ & $x_{1}$ & $b$ & $a$ & $e$ \\
\hline
\end{tabular}

The canonical representation is easily obtained if we write this table in terms of the numerical values. If we wish to verify isomorphism of two groups, we simply have to find the numerical tables and these have to coincide.

\section{Infinite Sets and Real Numbers}

In this section we will build the structure of real numbers, using the same principles of our construction of natural numbers. We simply have to extend our methods to the case of infinite sets. First of all, notice that any real number in the unit interval $(0,1]$ can be given in negative powers of 2 . For example, the number $\frac{1}{2}=2^{-1}$ and $\frac{3}{4}=2^{-1}+2^{-2}$.

We make a second observation. Consider the energy level graph of a sum, as in Figure 1. Notice that we can vertically displace the configuration of points, and still obtain a true statement. Now, what happens if we make a displacement into negative integers? The statement holds still, as in Figure 3 This is true because negative powers of two are still operated with the same rule. The expression $2^{n}+2^{n}=2^{n+1}$ holds for any integer $n$, not only positive integers. For example, if we wish to add the numbers $\frac{1}{2}+\frac{3}{4}$ we have $2^{-1}+2^{-1}+2^{-2}=2^{0}+2^{-2}=1+\frac{1}{4}$. We use this to our advantage in formalizing the concept of real number. 


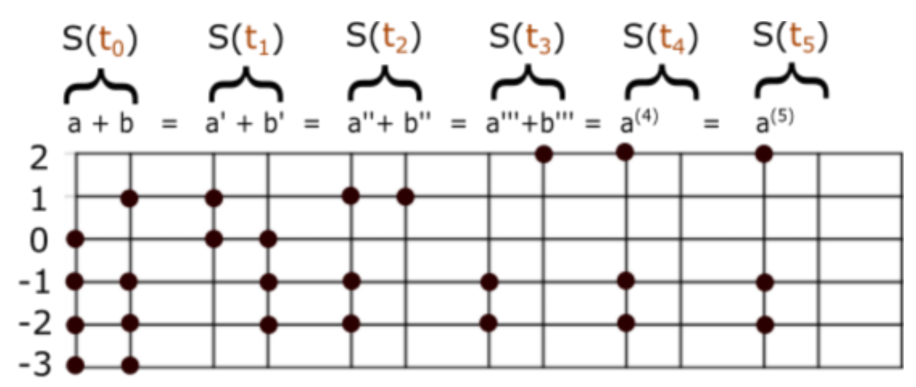

Figure 3: The energy level interpretation can be taken to negative levels. Particles occupying these levels represent negative powers of 2 . In Figure 1 this represented $15+23=38$. Here, we have the statement $1.875+2.875=4.75$.

We have seen how to represent natural numbers as hereditarily finite sets, and $\mathbb{N}=$ HFS. We know, because of the union axiom, that HFS $=\bigcup_{n} \oplus 1^{n}(0)$ is a set. In our first axiom we accept that the sub collection of any set, is also a set. This means that any infinite sub collection of HFS, is a set. In this section we will prove that these sets are the real numbers. We divide this section in three main parts.

1. Continuum $[0,1]$. Any real number in the unit interval is the sum of infinitely many negative powers of 2. Moreover, every infinite set of natural numbers defines a unique real number in the unit interval.

2. Real Numbers. We generalize the constructions of $\mathbb{N}$ and $[0,1]$ to represent positive real numbers as infinite subsets of $\mathbb{Z}$. Then, we give the structure of $\mathbb{R}$ to the set of infinite subsets of HFS.

3. Límits and Continuity. The concept of limit and continuity has a simple description in terms of our constructions. We give an initial description of Analysis, in terms of the order for natural numbers, $\mathbb{N}_{<}$.

We give a brief description of these developments, and leave some of the proofs for a separate publication on real numbers.

\subsection{Continuum $[0,1]$}

A real number $x \in(0,1]$ can be expressed as a sum of negative powers of 2 , so that $x=\sum_{i \in X} 2^{-i}$ for some set $X \subseteq \mathbb{N}$. The set $X \subset \mathbb{N}$ is the set number corresponding to $x$. The set number $X$ can be a finite set (for some rational numbers). However, notice that any rational number $x=\sum_{i=1}^{n} 2^{-i}$ can be seen as an infinite sum $x=\left(\sum_{i=1}^{n-1} 2^{-i}\right)+\left(\sum_{i=n}^{\infty} 2^{-i}\right)$. Thus, every $x \in(0,1]$ is represented by a unique infinite set of natural numbers greater than 0 . We use the symbol $\mathbb{N}_{1}=\{1,2,3, \ldots\}$ for the set of natural numbers greater than 0 . We have a bijection $\mathbb{N}_{\text {inf }} \rightarrow(0,1]$, where $\mathbb{N}_{\text {inf }}$ is the set of all infinite subsets of $\mathbb{N}_{1}$. We call these sets, infinite set numbers and they are ordered similarly to finite set numbers, but with one difference. The smaller powers of 2 represent larger numbers. For example, $2^{-5}<2^{-1}$. Instead of using the maximum of the set difference, now we look for the minimum. Therefore, we define $A<B$ if and only if

$\min (A \triangle B) \in B$

Notice that $1=\mathbb{N} \in \mathbb{N}_{\text {inf }}$. Let us verify this is a transitive order on $\mathbb{N}_{\text {inf }}$, because it is trivial to verify it is anti symmetric. Suppose $A<B$ and $B<C$. We know there exists an object $c_{0} \in C / B$ such that $c_{0}<b$ for every $b \in B / C$. We also know there is an object $b_{1} \in B / A$ such that $b_{1}<a$ for every $a \in A / B$. Suppose there exists $a_{2} \in A / C$ such that $a_{2}<c$ for every $c \in C / A$. We treat two cases and in each we arrive at a contradiction, proving $A<C$. 
Let us suppose $a_{2} \in B$. Then $c_{0}<a_{2}$. This means $c_{0}$ must be in $A$. This implies $b_{1}<c_{0}$. Thus, $b_{1} \in C / A$ and $a_{2}<b_{1}$ which is a contradiction.

Let us suppose $a_{2} \notin B$. This implies $b_{1}<a_{2}$. We know $b_{1} \notin A$ so that $b_{1} \in C$ implies $a_{2}<b_{1}$ which is a contradiction. Therefore, we must have $b_{1} \notin C$. Then, $c_{0}<b_{1}$. For $c_{0}<a_{2}$ to be true, we need $c_{0} \in A$. But, this would imply $b_{1}<c_{0}$, again a contradiction.

This proves our order on $\mathbb{N}_{\text {inf }}$ is transitive. Obviously, any two objects in $\mathbb{N}_{\text {inf }}$ are comparable in terms of this relation, <, because the symmetric difference is non empty for different set numbers $A \neq B$. Then, we know $\min (A \triangle B)$ exists because of the well order principle. We have ordered $\mathbb{N}_{\text {inf }}$ isomorphic to $(0,1]$. The real number, 1 , is the set $\mathbb{N}$. To include the real number 0 , in our order, we consider $\mathbb{N}_{\text {inf }}^{*}=\mathbb{N}_{\text {inf }} \cup\{\emptyset\}$. This is the set whose objects are the infinite subsets of $\mathbb{N}$, plus the empty set. Now we have the order of $[0,1]$ in terms of sets, where $0=\emptyset$ and every $x \in(0,1]$ is an infinite set of natural numbers. The most important aspect in the order of a continuum, is the the supremum property. This is what characterizes a continuum from a discrete order. Now we will show that the supremum exists, for our order $\mathbb{N}_{\text {inf }}^{*}$. Let $\mathbf{X} \subseteq \mathbb{N}_{\text {inf }}^{*}$; every element of $\mathbf{X}$ is an infinite set of natural numbers. Define $x_{1}=\min (\cup \mathbf{X})$ and $Y_{1}=\left\{A \in \mathbf{X} \mid x_{1} \in A\right\}$. Let

$$
x_{n+1}=\min \left(\bigcup Y_{n}-\left\{x_{i}\right\}_{i=1}^{n}\right),
$$

where $Y_{n}=\left\{A \in Y_{n-1} \mid x_{n} \in A\right\}$. The set number $\left\{x_{i}\right\}_{i} \in \mathbb{N}_{\text {inf }}^{*}$ is the supremum of $X$, by construction. This is shown in Figure 4 .

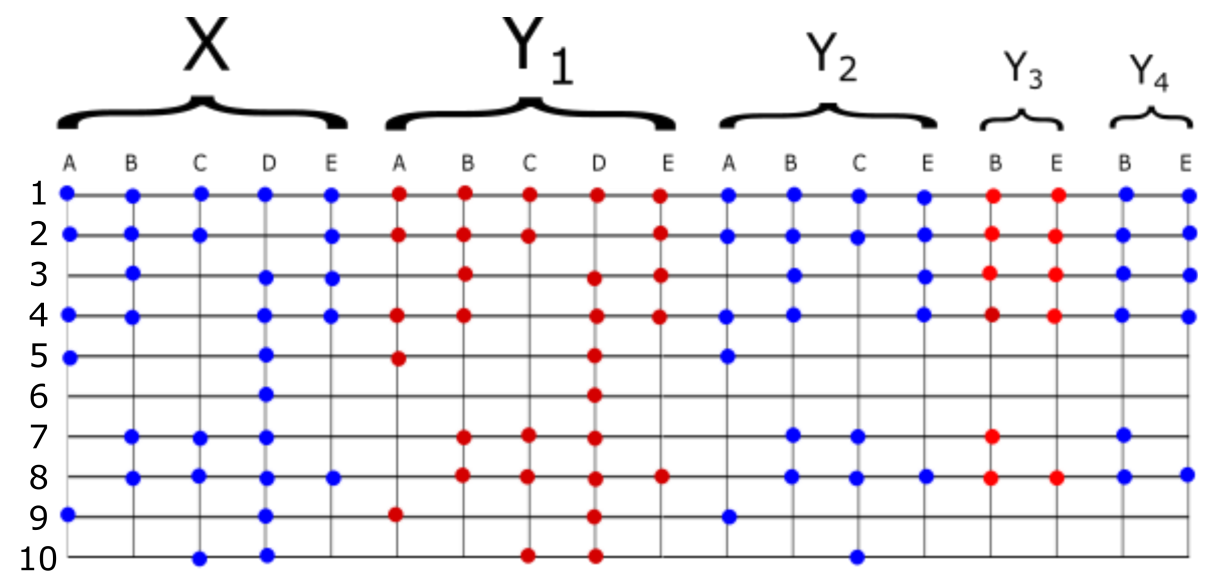

Figure 4: Here we represent the process of finding the supremeum of the family $X=\{A, B, C, D, E\}$. The elements of $X$ are set numbers in the unit interval. For example, $A=2^{-1}+2^{-2}+2^{-4}+2^{-5}+2^{-9}=0.845703125$.

The next step, after defining our order for infinite set numbers, is to define the addition of infinite set numbers. Let $r=s^{-1}$; the inverse function of $s$. Recall, this function subtracts 1 one unit to the elements of the argument. Given two infinite set numbers $A=\left\{a_{1}, a_{2}, \ldots\right\}$ and $B=\left\{b_{1}, b_{2}, \ldots\right\}$, let $A_{n}=\left\{a_{k}\right\}_{k=1}^{n}$ and $B_{n}=\left\{b_{k}\right\}_{k=1}^{n}$ be the sets of the first $n$ objects. Define

$$
A_{n} \oplus B_{n}=\left(A_{n} \triangle B_{n}\right) \oplus r\left(A_{n} \cap B_{n}\right)
$$

The addition $A \oplus B$ is the supremum of the finite sums,

$$
A \oplus B=\sup _{n}\left(A_{n} \oplus B_{n}\right) .
$$




\subsection{Real Numbers}

We can generalise our previous constructions of $\mathbb{N}$ and $[0,1]$, into a single structure, $\mathbb{R}_{0}^{+}$, based on the observation of Figure 3 . But, first we need to prove that the integers are sets. Take the integer $1 \in \mathbb{Z}$; it is the function $\oplus 1$. We know that finite function is a finite set of set numbers. Then, the function $\oplus 1$ is the object $\{\{1,4\},\{3,6\},\{5,8\},\{7,10\}, \ldots\}$. Thus, the integer $1 \in \mathbb{Z}$ is an infinite set number and, in particular, a set. The integer $2 \in \mathbb{Z}$ is the infinite set $\{\{1,6\},\{3,8\},\{5,10\},\{7,12\}, \ldots\} \in \mathbb{N}_{\text {inf }}^{*}$, etc. The negative integer $-1 \in \mathbb{Z}$ is the object $\{\{\{3,2\},\{5,4\},\{7,6\},\{9,8\}, \ldots\}$. The negative integer $-2 \in \mathbb{Z}$ is the object $\{\{\{5,2\},\{7,4\},\{9,6\},\{11,8\}, \ldots\}$, etc. Now we wish to show that the collection of integers is a set. Given that $\mathbb{Z}$ is a sub collection of $\mathbb{N}_{i n f}^{*}$, it is sufficient to prove $\mathbb{N}_{i n f}^{*}$ is a set (because of Axiom 1). We know the elements of $\mathbb{N}_{\text {inf }}^{*}$ are sets. But we can not go any further with our axioms. Our axioms allow us to build sets using union and intersection, and sub collections. If we take the union of the infinite set numbers we get $\mathbb{N}=\bigcup_{i \in \mathbb{N}_{i n f}^{*}} i$. We can not prove $\mathbb{N}_{i n f}^{*}$ is a set. We need a new axiom.

Axiom 3. Let $X$ a collection of constructed sets, then $X$ is a set.

Here we have to be very careful to avoid the commonly known paradox of the set of all sets, so let us be clear on this. If we have a collection of concrete sets, then that collection is a set. We will see the implications of this in the last section. Intuitively, every set is in a larger set. For now, we only care that $\mathbb{N}_{i n f}^{*}$ is a set because it is a collection of sets. Thus we have proven $\mathbb{Z}$ is a set. Let $\overline{\mathbb{Z}} \subset \mathbb{N}_{\text {inf }}^{*}$ the set whose objects are subsets of $\mathbb{Z}$, that are bounded above. Put differently, $A \in \overline{\mathbb{Z}}$ if and only if $A \subset \mathbb{Z}$ and $\max (A)$ exists. We can treat $A$ as a positive real number because a positive real number is well represented by a sum of integer powers of 2 . The non negative integers represent the whole part of the real number, while the negative integers are the decimal part of the real number, as in Figure 3. This simply means $A \cap \mathbb{N}$ is the whole part of $A$, and $A \cap-\mathbb{N}$ is the decimal part. The set of all non negative real numbers is $\mathbb{R}_{0}^{+}=\overline{\mathbb{Z}} \cup\{\emptyset\}$. Two positive real numbers are order related $A<B$ if and only if $\max (A \triangle B) \in B$. The addition is defined as before by $A \oplus B=(A \triangle B) \oplus s(A \cap B)$. The supremum can also be found in this structure of sets. At this point, we can proceed to build the negative real numbers using the same technique we used to build the negative integers. Every $A \in \mathbb{R}_{0}^{+}$is identified with a function $\oplus A: \mathbb{R}_{0}^{+} \rightarrow A$. Namely, we build a new set of positive real numbers, which will be the bijections $\mathbb{R}_{0}^{+} \rightarrow A$. We can then identify negative real numbers as the inverse functions of these. We do not focus on this construction, in this work, because we use a different path to define the structure of all real numbers.

Our alternative method of building the set of real numbers, $\mathbb{R}$, does not depend on integers, only on natural numbers. Using our construction of the unit interval $[0,1]$, we can represent every real number as an object in $\mathbb{N}_{\text {inf }}^{*}$.

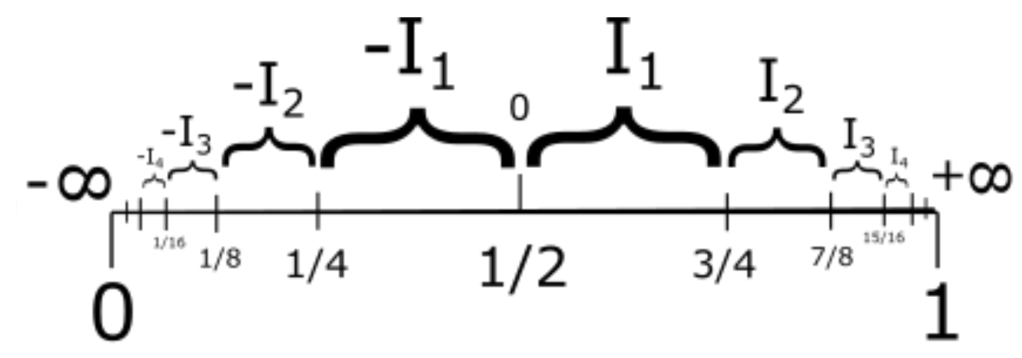

Figure 5: We use the fact that $\mathbb{R}$ is bijective to any interval $\left(\frac{n}{2^{k}}, \frac{n+1}{2^{k}}\right]$. Under this representation, the real number $0 \in \mathbb{R}$ is the set $\{2,3,4,5, \ldots\}$. We also have $-\infty \in \mathbb{R}=\emptyset$ and $+\infty=\mathbb{N}_{1}$

Each of the positive intervals $I_{1}=(0,1], I_{2}=(1,2], \ldots$, and negative intervals $-I_{1}=(-1,0],-I_{2}=(-2,-1], \ldots$, is isomorphic to the interval $\left(\frac{n}{2^{k}}, \frac{n+1}{2^{k}}\right]$, for any $n \leq 2^{k}-1$ in $\mathbb{N}$. Intuitively, what we will do is to compress and fit all the intervals $I_{i}$, into the unit interval, as in Figure 5 . The interval $I_{1} \subset \mathbb{R}$ is identified with the interval $\left(\frac{1}{2}, \frac{3}{4}\right] \subset[0,1]$. The interval $I_{2} \subset \mathbb{R}$ is the interval $\left(\frac{3}{4}, \frac{7}{8}\right] \subset[0,1]$, etc. The negative interval $-I_{1} \subset \mathbb{R}$ is the interval $\left(\frac{1}{4}, \frac{1}{2}\right] \subset[0,1]$, etc. 
Let $X \in[0,1]=\mathbb{N}_{i n f}^{*}$. Notice that a number $x \in\left(\frac{1}{2}, \frac{3}{4}\right] \in \mathbb{N}_{i n f}^{*}$ is an infinite set number such that $1 \in X$ and $2 \notin X$. A set number $x \in\left(\frac{3}{4}, \frac{7}{8}\right] \in \mathbb{N}_{\text {inf }}^{*}$ is an infinite set number such that $1 \in X$ and $2 \in X$, but $3 \notin X$, etc. A set number $X \in\left(\frac{1}{4}, \frac{1}{2}\right] \in \mathbb{N}_{\text {inf }}^{*}$ is an infinite set number such that $1 \notin X$ and $2 \in X$. A set number $X \in\left(\frac{1}{8}, \frac{1}{4}\right] \in \mathbb{N}_{\text {inf }}^{*}$ is an infinite set number such that $1,2 \notin X$ and $3 \in X$, etc. This has a simple form, that we can easily interpret in defining the set of all real numbers. Let $X=\left\{1,2,3, \ldots, n, k_{1}, k_{2}, k_{3}, \ldots\right\} \in \mathbb{N}_{i n f}^{*}$, where $3 \leq n+2 \leq k_{1}<k_{2}<k_{3}<\cdots$, then we will say $X$ is positive real number. A negative real number is $X=\left\{n, k_{1}, k_{2}, k_{3}, \ldots\right\}$ with $3 \leq n+1 \leq k_{1}<k_{2}<k_{3}<\cdots$. This simply means we will define a positive real number with whole part equal to $n$, as an infinite set number $X$ with $1,2, \ldots, n+1, \in X$ and $n+2 \notin X$. A negative real number with whole part equal to $-n$ is an infinite set number $X$ with $\min (X)=n+1$. The decimal part will be given by the remaining objects $k_{1}, k_{2}, k_{3} \ldots$. We can immediately differentiate a set positive set number from a negative set number. For example, The set number $\{1,2,3,4,10,11,12,13, \ldots\}$ is positive with whole part equal to 3 . The set number $\{5,10,11,12,13, \ldots\}$ is negative with whole part equal to -3 . The set number $\{1,2,6,7,8, \ldots\}$ has whole part equal to 1 , while $\{6,8,9,10, \ldots\}$ has whole part equal to -4 .

How do we find the decimal part of a set number, in this context? We simply used the first natural numbers as place holders for identifying the whole part. Let $X \in \mathbb{N}_{\text {inf }}^{*}$ be an infinite set number. The objects $k_{1}, k_{2}, k_{3}, \ldots$ are representing the decimal part of $X$. We assign $X$ the decimal part $r^{n+1}\left(\left\{k_{1}, k_{2}, k_{3}, \ldots\right\}\right)$. Let us look at the problem backwards, to better understand why. If we want to store the information of a real number $x \in \mathbb{R}$ as an infinite set number, how would we do it? We already saw that we need the first $n$ natural numbers to determine the whole. But then we still have infinitely many natural numbers left to determine the decimal part. So all we have to do, is displace the decimal part $n+1$ places, so that the decimal part and whole part do not interfere. Notice that in the case of positive real numbers, we need to leave one natural number out, as a queue that the whole part ends there, and now we start with the representation of the decimal part. Displacement up, $n+1$ times, is equivalent to applying $s^{n+1}$. Now, to recover the decimal part, we have to displace the $k_{i}$ 's back $n+1$ times by applying $r^{n+1}$.

Let us look at this in formal manner. For every $x \in \mathbb{N}_{\text {inf }}^{*}$, the numbers $\min (x)$ and $\min \left(x^{c}\right)$ are well defined, because of the well ordering principle. Exactly one of these two is equal to 1 and the other is larger than 1. A positive real number is an infinite set number with $\min (x)=1$. A negative real number is an infinite set number with $\min \left(x^{c}\right)=1$. More specifically, if $0<x \leq 1$ then $\min \left(x^{c}\right)=2$, and if $-1<x \leq 0$ then $\min (x)=2$. The equality $\min \left(x^{c}\right)=3$ is equivalent to $1 \leq x<2$, and $\min (x)=3$ is equivalent to $-2<x \leq-1$. If $2<x \leq 3$ then we have $\min \left(x^{c}\right)=4$, and if $-3<x \leq-2$ then we have $\min (x)=4$. In general, $x \in(n-1, n]$ if and only if $\min \left(x^{c}\right)=n+1$, and $x \in(-n,-(n-1)]$ if and only if $\min (x)=n+1$.

For example, the decimal part of $\pi$ is given by the set

$$
\{3,6,11,12,13,14,15,16,18, \ldots\} .
$$

because it is equal to $2^{-3}+2^{-6}+2^{-11}+2^{-12}+2^{-13}+2^{-14}+\cdots$. Therefore, the numbers $\pi$ and $-\pi$ are represented by

$$
\begin{aligned}
\pi & =\{1,2,3,4,3+5,6+5,11+5,12+5,13+5,14+5,15+5,16+5,18+5, \ldots\} \\
& =\{1,2,3,4,8,11,16,17,18,19,20,21,23, \ldots\} \\
-\pi & =\{5,3+5,6+5,11+5,12+5,13+5,14+5,15+5,16+5,18+5, \ldots\} \\
& =\{5,8,11,16,17,18,19,20,21,23, \ldots\}
\end{aligned}
$$

The set of infinite set numbers $\mathbb{N}_{\text {inf }}^{*}$ is $\mathbb{R}$. Real numbers and natural numbers are different types of sets. Natural numbers are the elements of HFS, while real numbers are the infinite subsets of HFS. Of course we can make adequate definitions for addition of real numbers, in this definition. 


\subsection{Limits and Continuity}

Now we have the task of finding suitable and practical expressions of the concepts of analysis. We begin by defining the concept of limit point. Let $P, X \in \mathbb{N}_{\text {inf }}^{*}$ two infinite set numbers. Intuitively, these two objects are close, if their first terms coincide. Take as an example the set numbers $P=\{2,4,5,8,9,10,11,12,13, \ldots\}=2^{-2}+2^{-4}+2^{-5}+2^{-8}+2^{-9}+2^{-10}+\ldots$ and $X=\{2,4,5,8,9,14,15,16,17 \ldots\}=2^{-2}+2^{-4}+2^{-5}+2^{-8}+2^{-9}+2^{-14}+\ldots$. They are relatively close because the first terms (the largest terms) coincide. So we know we need to ask that the first elements coincide, for two numbers to be close. Another way of saying this is that $\min (P \triangle X)$ is a large number. The larger $\min (P \triangle X)$, the larger the elements of $P \triangle X$ become, making the smaller powers (larger terms) coincide. Remember, that in the decimal part, larger natural numbers represent the smaller terms of the real number.

Let us give a formal definition of this. Let $P \in \mathbb{N}_{i n f}^{*}$ an infinite set number, and let $\mathbf{X}$ a set of infinite set numbers. We say $P$ is a limit point of $\boldsymbol{X}$ if there exists $X_{N} \in \mathbf{X}$ such that $\min \left(P \triangle X_{N}\right)>N$, for every $N \in \mathbb{N}$. There is one exception to this definition. When we started to describe real numbers as infinite set numbers we noticed some real numbers had decimal part that could be expressed as sum of finite many negative powers of 2 . We will treat these numbers separately in defining limit points. Suppose $P \in \mathbb{N}_{\text {inf }}^{*}$ is an infinite set number that has finite representation $P=\left\{p_{1}, p_{2}, \ldots, p_{k}\right\}$, where $p_{1}<p_{2}<\cdots<p_{k}$. That is to say, we can write it as $P=\left\{p_{1}, p_{2}, \ldots, p_{k-1}, p_{k}+1, p_{k}+2, p_{k}+3, \ldots\right\}$. We simply replace the last term $2^{-p_{k}}$ with infinite terms, $2^{-\left(p_{k}+1\right)}+2^{-\left(p_{k}+2\right)}+2^{-\left(p_{k}+3\right)}+\ldots$. We give aset of infinite set numbers that get as close to $P$ as we would like, using larger numbers than $P$. Let $X_{1}=\left\{p_{1}, p_{2}, \ldots, p_{k}, p_{k}+1\right\}$, and $X_{2}=\left\{p_{1}, p_{2}, \ldots, p_{k}, p_{k}+2\right\}$. In general define $X_{i}=\left\{p_{1}, p_{2}, \ldots, p_{k}, p_{k}+i\right\}$. These set numbers $X_{i}$ are getting closer to $P$ but our rule is not satisfied. The minimum element of the symmetric difference is not getting larger. In fact, it is constant, $\min \left(P \triangle X_{i}\right)=p_{k}$. Therefore, we must make a different definition for this case. If $P$ is an infinite set number with finite representation, then we say $P$ is a limit point of $\mathbf{X}$ if for every $N \in \mathbb{N}$, there exists $X_{N} \in \mathbf{X}$ such that $X_{N}=\left\{p_{1}, p_{2}, \ldots, p_{k}, p_{k}+N, p_{n_{1}}, p_{n_{2}}, \ldots\right\}$, where $p_{k}+N<p_{n_{1}}<p_{n_{2}}<\cdots$.

In both cases we are requiring an infinite set number $X_{N}$ such that $\left|P-X_{N}\right| \leq \frac{1}{2^{N}}=\{N\}$. Let $P, X$ two infinite set numbers with their whole parts equal and suppose their decimal parts coincide in the first elements. We have

$$
\begin{aligned}
& P=2^{m_{1}}+2^{m_{2}}+\ldots+2^{m_{k}}+2^{n_{1}}+2^{n_{2}}+2^{n_{3}}+\ldots+2^{N}+2^{\alpha_{1}}+2^{\alpha_{2}}+2^{\alpha_{3}}+\ldots \\
& X=2^{m_{1}}+2^{m_{2}}+\ldots+2^{m_{k}}+2^{n_{1}}+2^{n_{2}}+2^{n_{3}}+\ldots+2^{N}+2^{\beta_{1}}+2^{\beta_{2}}+2^{\beta_{3}}+\ldots
\end{aligned}
$$

where $m_{1}<m_{2}<\ldots<m_{k}<n_{1}<n_{2}<\ldots<N<\alpha_{1}<\alpha_{2}<\cdots$ and $N<\beta_{1}<\beta_{2}<\cdots$. The numbers $m_{i}$ determine the whole part and $n_{i}, N$ are the elements that coincide in the decimal part (the first negative powers of 2 that coincide). Then, the difference $|P-X|<\frac{1}{2^{N-\left(m_{k}+1\right)}}$ is bounded by $\frac{1}{2^{N-\left(m_{k}+1\right)}}$.

We see that infinite set numbers with finite representations can be handled in another, informal, manner. For example, $1 / 2 \in$ $[0,1]$ has the representations $\{1\}=\{2,3,4, \ldots\}$. We know $P=1 / 2$ should be a limit point of the set $\mathbf{X}=\left\{A_{1}, A_{2}, A_{3}, A_{4}, \ldots\right\}$ where $A_{i}$ are

$$
\begin{aligned}
& A_{1}=1=\{1,2,3,4,5,6, \ldots\} \\
& A_{2}=3 / 4=\{1,3,4,5,6,7, \ldots\} \\
& A_{3}=5 / 8=\{1,4,5,6,7,8, \ldots\} \\
& A_{4}=9 / 16=\{1,5,6,7,8,9, \ldots\}
\end{aligned}
$$


If $P=\{2,3,4,5, \ldots\}$ then $\min \left(P \triangle A_{i}\right)=1$, for every $A_{i}$. But, if we use the finite representation $P=\{1\}$, then the symmetric differences are: $P \triangle A_{1}=\{2,3,4, \ldots\}, P \triangle A_{2}=\{3,4,5, \ldots\}, P \triangle A_{3}=\{4,5,6, \ldots\}, P \triangle A_{4}=\{5,6,7, \ldots\}, \ldots$ In effect, complying with our condition that for every $N \in \mathbb{N}$ there exists $X_{N} \in \mathbf{X}$ such that $\min \left(P \triangle X_{N}\right)>N$.

If $P$ has finite representation and we where to get closer to $P$, using smaller numbers, we can not have the same problem that we had when we were getting closer from above. For example, take $P=1 / 2=\{2,3,4,5, \ldots\}$ and the set $\mathbf{X}=\left\{A_{1}, A-2, \ldots\right\}$ defined by

$$
\begin{array}{ll}
A_{1}=3 / 8 & =\{2,4,5,6,7,8 \ldots\} \\
A_{2}=7 / 16 & =\{2,3,5,6,7,8 \ldots\} \\
A_{3}=15 / 32 & =\{2,3,4,6,7,8 \ldots\} \\
A_{4}=31 / 64 & =\{2,3,4,5,7,8 \ldots\} \\
A_{5}=63 / 128 & =\{2,3,4,5,6,8 \ldots\}
\end{array}
$$

We can easily verify that for every $N \in \mathbb{N}$ there exists $X_{N}$ such that $\min \left(P \triangle X_{N}\right)>N$. We have $P \triangle A_{1}=\{3\}, P \triangle A_{2}=\{4\}$, $P \triangle A_{3}=\{5\}, P \triangle A_{4}=\{6\}, P \triangle A_{5}=\{7\}, \ldots$

Continuity is described in terms of the order of natural orders, consequently. In the next section we will provide a formal definition for real function. We use it provisionally, for the sake of illustration.

Definition 12. Let $f: \boldsymbol{A} \subseteq \mathbb{R} \rightarrow \boldsymbol{B} \subseteq \mathbb{R}$ a real function, and let $p$ a limit point of the domain $\boldsymbol{A}$. We say $f$ has limit point $p$, and the limit is equal to $q$, if and only if for every $N \in \mathbb{N}$ there exists $M \in \mathbb{N}$ such that $\min (p \triangle x)>M$ implies $\min (f(p) \triangle q)>N$.

The function is continuous in $p$ if and only if for every $N \in \mathbb{N}$ there exists $M \in \mathbb{N}$ such that $\min (p \triangle x)>M$ implies $\min (f(p) \triangle f(x))>N$.

The theory of convergence and topological aspects of $\mathbb{R}$ are expressed directly in terms of the order of natural numbers. Using these general indications and the subtraction algorithm, given in [I], it is possible to define the derivative. We can treat the derivative in two ways. If we use the subtraction algorithm we can define the derivative in the traditional manner to find the numerical value $f^{\prime}(p)$. If, however, we only wish to prove the existence of the derivative, we will have an alternative definition of a discrete derivative. We know the quotient of two powers of 2 is obtained by subtracting the powers, $\frac{2^{n}}{2^{m}}=$ $2^{n-m}$. The derivative of $f$ exists at $p$ if there exists $M \in \mathbb{N}$ such that $\min (f p \triangle f x)+M>\min (p \triangle x)$ for every $x \in \mathbf{A}$. In the case that $\min (f p \triangle f x)>\min (p \triangle x)$ for every $x \in \mathbf{A}$, we have $0 \leq\left|f^{\prime}(p)\right|<1$. The derivative is exactly equal to 0 when $\min (f p \triangle f x)-\min (p \triangle x)$ is not bounded; $\min (p \triangle x)$ goes to infinity but $\min (f p \triangle f x)$ goes to infinity faster so that $\min (f p \triangle f x)-\min (p \triangle x)$ goes to infinity. If $\min (f p \triangle f x)=\min (p \triangle x)$ for every $x \in \mathbf{A}$ then $\left|f^{\prime}(p)\right|=1$. If we need to add $M \in \mathbb{N}$ to get $\min (f p \triangle f x)+M>\min (p \triangle x)$ for every $x \in \mathbf{A}$ we have $\left|f^{\prime}(p)\right|>1$.

The discrete derivative is a criteria for the existence and absolute value of the magnitude of the derivative. In exchange, for not knowing the exact numerical value of the derivative, we can say that finding the discrete derivative is computationally much faster. We are substituting the quotient $\frac{f p-f x}{p-x}$ of floating point numbers, with finding the difference of natural numbers, $|\min (f p \triangle f x)-\min (p \triangle x)|$. The end result is that instead of having to calculate two subtractions and one division of real numbers, we find the minimum element for two sets of natural numbers and the difference of these natural numbers. 


\section{Trees and Type Theory}

In this section we will give an account of how to build and represent general objects used in modern mathematics. This will be a very superficial description but we will show enough of these constructions to be clear on the extent of constructions possible. We also show how this universe of sets can be well represented in terms of trees. We also give a brief description of the theory of types this axiomatic base provides. We see how to give a consistent hierarchy of types and universes.

\subsection{Basic Objects In Mathematics}

Ordered pairs, and finite sets of ordered pairs, are natural numbers. Now let us define an ordered $n$-tuple of natural numbers. Recall that to define an ordered pair of natural numbers we used a simple trick. We used even and odd natural numbers to tell apart our first component from the second. One might initially want to go about this in the following manner. If we wish to well represent ordered 3-tuples we could use numbers $\{1,4,7,10, \ldots, 3 k-2, \ldots\}$ to represent the first component, then we use $\{2,5,8,11, \ldots, 3 k-1, \ldots\}$ to represent the second component, and multiples of three, $\{3,6,9,12, \ldots, 3 k, \ldots\}$ to represent the third component. This will give us a table similar to [22), when we defined ordered pairs.

$\begin{array}{cccc}X & 3 k-2 & 3 k-1 & 3 k \\ 0 & 1 & 2 & 3 \\ 1 & 4 & 5 & 6 \\ 2 & 7 & 8 & 9 \\ 3 & 10 & 11 & 12 \\ 4 & 13 & 14 & 15 \\ 5 & 16 & 17 & 18 \\ 6 & 19 & 20 & 21 \\ \vdots & \vdots & \vdots & \vdots\end{array}$

The ordered 3-tuple $(0,0,0)$ is the set number $2^{1}+2^{2}+2^{3}=\{1,2,3\}$. We also have $(1,2,3)$ equal to $2^{4}+2^{8}+2^{12}=\{4,8,12\}$. If we want to represent 4-tuples we would have to come up with a new table

$\begin{array}{ccccc}X & 4 k-3 & 4 k-2 & 4 k-1 & 4 k \\ 0 & 1 & 2 & 3 & 4 \\ 1 & 5 & 6 & 7 & 8 \\ 2 & 9 & 10 & 11 & 12 \\ 3 & 13 & 14 & 15 & 16 \\ 4 & 17 & 18 & 19 & 20 \\ 5 & 21 & 22 & 23 & 24 \\ 6 & 25 & 26 & 27 & 28 \\ \vdots & \vdots & \vdots & \vdots & \vdots\end{array}$

This has two big disadvantages that will become clear with our second method of defining ordered $n$-tuples. The first is quite obvious. With this first method we can not define an infinite sequence of natural numbers. 
The easiest way to solve this is by going back to the definition of ordered pairs. We have another important table we can use. The sets given in (6) are of great importance in the constructions of this section. We include it again, for reference.

$$
\begin{aligned}
(0,)= & \left\{6,18,66,258,1026, \ldots, 2+2^{2(n+1)}, \ldots\right\} \\
(1,)= & \left\{12,24,72,264,1032, \ldots, 8+2^{2(n+1)}, \ldots\right\} \\
(2,)= & \left\{36,48,96,288,1056, \ldots, 32+2^{2(n+1)}, \ldots\right\} \\
\vdots & \vdots \\
(m,)= & \left\{2^{2 m+1}+4,2^{2 m+1}+16, \ldots, 2^{2 m+1}+2^{2(n+1)}, \ldots\right\}
\end{aligned}
$$

We give a definition of ordered pair that supersedes the one given before. To define an ordered pair of natural numbers it will only be necessary to use the first two sets $(0$,$) and (1$,$) . If we take one object from each of these sets, we can differentiate$ them and still have each one represent an arbitrary natural number. The ordered pair $(i, j)$, of natural numbers $i, j$, is the set number $\left\{2^{1}+2^{2(i+1)}, 2^{3}+2^{2(j+1)}\right\}$. We include the $i+1$-st element of $(0$,$) to show that i$ is in the first component. We include the $j+1$-st element of $(1$,$) to indicate j$ is in the second component. For example, the ordered pair $(0,0)$ is the set number $2^{6}+2^{12}=\{6,12\}$. The ordered pair $(0,1)$ is $2^{6}+2^{24}=\{6,24\}$. We are defining data types. Now if we want to define an infinite sequence $S$, of natural numbers, we can easily do this. Take one element from each set $n_{k} \in(k$,$) , for k \in \mathbb{N}$. Then, $2^{1}+2^{2\left(n_{1}+1\right)} \in S$ means $n_{1}$ is the first natural number of the sequence. The second number is given by $2^{3}+2^{2\left(n_{2}+1\right)} \in S$, and so on. The set number $\left\{2^{1}+2^{2\left(n_{1}+1\right)}, 2^{3}+2^{2\left(n_{2}+1\right)}, 2^{5}+2^{2\left(n_{3}+1\right)}, \ldots\right\}$ represents the sequence $\left(n_{1}, n_{2}, n_{3}, \ldots\right)$. For example, the sequence $(1,3,2,5,4,7,6,9,8,11,10,13,12, \ldots)$ is given by the infinite set number $\left\{2+2^{2(1+1)}, 8+2^{2(3+1)}, 32+2^{2(2+1)}, 128+2^{2(5+1)}, \ldots\right\}=$ $\{18,264,96,4224, \ldots\}$. Of course, to define a finite sequence, an $k$-tuple, we use the first $k$ sets, $(1),,(2),, \ldots(k$,$) . A finite$ sequence of natural numbers is a natural number $\left\{2^{1}+2^{2\left(n_{1}+1\right)}, 2^{3}+2^{2\left(n_{2}+1\right)}, 2^{5}+2^{2\left(n_{3}+1\right)}, \ldots, 2^{2 k+1}+2^{2\left(n_{k}+1\right)}\right\}$. An ordered pair is a set number $(A, B)$ where $A \in(0$,$) and B \in(1$,$) . We can easily describe functions \mathbb{N} \rightarrow \mathbb{N}$ as an infinite set of ordered pairs. A function of this form is a set number

$$
\left\{\left\{6, B_{1}\right\},\left\{18, B_{2}\right\},\left\{66, B_{3}\right\},\left\{258, B_{4}\right\}, \ldots\right\}
$$

where $B_{i}$ are elements of $(1$,$) . If the B_{i}$ are all distinct, we have an injection. If every element of $(1$,$) is a B_{i}$ the function is onto $\mathbb{N}$. This represents functions $\mathbb{N} \rightarrow \mathbb{N}$ as real numbers.

How would we go on about representing a sequence of real numbers? The same question stated differently, How can we represent a sequence of infinite sets of natural numbers? We wish to find the best way of storing and rescuing the information that determines a sequence $\left(r_{1}, r_{2}, r_{3}, \ldots\right)$ where each $r_{i}=\left\{n_{1}^{i}, n_{2}^{i}, n_{3}^{i}, \ldots\right\}$ is a real number. Use the set $(0$,$) to represent the$ elements of $r_{1}$. Use the set $(1$,$) to represent the elements of r_{2}$, etc. We have $2^{2(i)+1}+2^{2\left(n_{j}^{i}+1\right)} \in\left(r_{1}, r_{2}, \ldots\right)$ if and only if $n_{j}^{i} \in r_{i}$. The infinite sequence of real numbers, $\left(r_{1}, r_{2}, \ldots\right)$, is represented by the real number $\bigcup_{i} r_{i}$. The union of all the $r_{i}$ 's is a real number that represents the infinite sequence $\left(r_{1}, r_{2}, \ldots\right)$; it is an infinite set number with infinitely many objects from each set $(i$,$) . Actually, any set number with infinitely many elements of each (i$,$) is representing a unique sequence of real numbers. If$ we have an infinite set $X \subset(0$,$) this determines a real number. The set X=\left\{2+2^{2\left(x_{1}+1\right)}, 2+2^{2\left(x_{2}+1\right)}, 2+2^{2\left(x_{3}+1\right)}, \ldots\right\}$ determines the real number $X^{*}=\left\{x_{1}, x_{2}, x_{3}, \ldots\right\}$. In the same manner, we can have an infinite set $Y=\left\{8+2^{2\left(y_{1}+1\right)}, 8+2^{2\left(y_{2}+1\right)}, 8+\right.$ $\left.2^{2\left(y_{3}+1\right)}, \ldots\right\} \subset(1$,$) determine the real number Y^{*}=\left\{y_{1}, y_{2}, y_{3}, \ldots\right\}$. Observe that the infinite set number $X^{*} \cup Y^{*}$ is a real number, whose objects are in $(0,) \cup(1$,$) , and we can distinguish the objects of (0$,$) from the objects of (1$, ). The objects in $(0$,$) give us the first component, and the second component is given by the elements of (1,). Thus, we are able to represent$ the ordered pair of real numbers, $\left(X^{*}, Y^{*}\right)$, as a single real number $X \cup Y$. If we wish to represent ordered 3-tuples of real numbers, we can do so by using (2, ). The ordered 3-tuple $\left(X^{*}, Y^{*}, Z^{*}\right)$ is the real number $X \cup Y \cup Z$ where $Z^{*}=\left\{z_{1}, z_{2}, z_{3}, \ldots\right\}$ and $Z=\left\{32+2^{2\left(z_{1}+1\right)}, 32+2^{2\left(z_{2}+1\right)}, 32+2^{2\left(z_{3}+1\right)}, \ldots\right\} \subset(2$,$) . An infinite sequence of real numbers x_{1}, x_{2}, \ldots$ is represented by a single real number. We are giving an injective function from the set of all real sequences to the set of real numbers. 
Now we know how to well represent sequences of real numbers, and we can also well represent functions $\mathbb{N} \rightarrow \mathbb{N}$ as real numbers. Consequently, we can represent a sequence $\left(f_{1}, f_{2}, \ldots\right)$, of functions $f_{i}: \mathbb{N} \rightarrow \mathbb{N}$. We have provided a method of defining ordered pairs, that is a more powerful definition than the first, because it allows us to represent infinite sequences, of $\mathbb{N}$ and $\mathbb{R}$, as real numbers. It also shows that we can represent an infinite function, $\mathbb{N} \rightarrow \mathbb{N}$, as a real number. Let us find a way of representing sequences of sequences. Start with the simplest kind, a sequence $T=\left(S_{1}, S_{2}, \ldots\right)$ of sequences, $S_{i}$, of natural numbers. We use subsets of $(i$,$) to find these representations. We will work with a subset of (0$,$) to represent the first$ sequence $S_{1}=\left(n_{1}^{1}, n_{2}^{1}, n_{3}^{1}, \ldots\right)$. We have

$$
2+2^{2\left(\left(2+2^{2\left(n_{1}^{1}+1\right)}\right)+1\right)}, 2+2^{2\left(\left(2+2^{2\left(n_{2}^{1}+1\right)}\right)+1\right)}, \ldots \in T
$$

for every $i=1,2,3, \ldots$. We use a subset of $(1$,$) to represent the second sequence. If S_{2}=\left(n_{1}^{2}, n_{2}^{2}, n_{3}^{2}, \ldots\right)$ is the second sequence, then we have

$$
8+2^{2\left(\left(2+2^{2\left(n_{1}^{2}+1\right)}\right)+1\right)}, 8+2^{2\left(\left(2+2^{2\left(n_{2}^{2}+1\right)}\right)+1\right)}, \ldots \in T
$$

for every $i=1,2,3 \ldots$ We use the set $(k$,$) to represent the sequence S_{k}$. We are able to reconstruct the sequence of sequences, from the real number

$$
T=\left\{2+2^{2\left(\left(2+2^{2\left(n_{1}^{1}+1\right)}\right)+1\right)}, 2+2^{2\left(\left(2+2^{2\left(n_{2}^{1}+1\right)}\right)+1\right)}, \ldots, 8+2^{2\left(\left(8+2^{2\left(n_{1}^{2}+1\right)}\right)+1\right)}, 8+2^{2\left(\left(2+2^{2\left(n_{2}^{2}+1\right)}\right)+1\right)}, \ldots\right\}
$$

The main difference between natural numbers and real numbers is that a real function is not represented by a real number, as natural functions are represented by natural numbers. There is still good news, because a real function is represented by a set of real numbers. Above, we have defined an ordered pair of real numbers, so now we can define a real function. We know that a function is a collection of components $f_{x}=(x, f x)$. We also know every ordered pair of real numbers is a real number, $f_{x} \in \mathbb{R}$. Therefore, the function $f$ can be represented by a set of real numbers $\left\{f_{x}\right\}_{x \in \mathbb{R}}$. A real function is set of real numbers,

$$
f=\left\{\left\{a_{1}^{x}, a_{2}^{x}, \ldots, b_{1}^{x}, b_{2}^{x}, \ldots\right\}\right\}_{x \in \mathbb{R}}
$$

where $x=\left\{a_{i}^{x}\right\}_{i} \subset(0$,$) and f(x)=\left\{b_{i}^{x}\right\}_{i} \subset(1$,$) . This means f_{x}=x \cup f(x)=\left\{a_{1}^{x}, a_{2}^{x}, \ldots, b_{1}^{x}, b_{2}^{x}, \ldots\right\}$. The function is injective if $f(x) \cap f(y)=\emptyset$ for $x \neq y$. The function $f$ is onto $\mathbb{R}$ if for every infinite subset $A \subset(1$,$) , there exists an object x \in \mathbb{R}$ such that $A=f_{x} \cap(1$,$) . A real function is bijective if for every infinite subset A \subset(1$,$) there exists exactly one x \in \mathbb{R}$ such that $A=f_{x} \cap(1$,$) .$

Next, in line of mathematical objects are sequences of real functions. A sequence of real functions is well represented by a set of real numbers. That is to say, we are able to well assign a set of real numbers to a sequence of real functions $\left(f_{1}, f_{2}, \ldots\right)$. Just as we use $(0$,$) and (1$,$) to define a function f_{1}$, we can also use $(2$,$) and (3$,$) to define a function f_{2}$. We use $(4$,$) and$ $(5$,$) to define a function f_{3}$, etc. There is another consequence of representing a real function as a set of real numbers. Since a function $\mathbb{R} \rightarrow \mathbb{R}$ is a set of real numbers, we can also represent functions of the form $\mathbb{R} \rightarrow(\mathbb{R} \rightarrow \mathbb{R})$ as sets of real numbers. In general, for any finite amount of iterations, an object $\mathbb{R} \rightarrow(\mathbb{R} \rightarrow(\mathbb{R} \rightarrow(\mathbb{R} \rightarrow \cdots(\mathbb{R} \rightarrow \mathbb{R}) \cdots))$ ) is a set of real numbers. 


\subsection{Trees}

We have seen that natural numbers are the finite sets we can build recursively with the function $\oplus 1$. These sets can be well represented by finite tree structures. We will use trees to represent natural numbers first, then all types of objects. Our concept of set is equivalent to the concept of tree, we will define. A finite set number is an object that contains smaller set numbers. Every element of that set is in turn a set of set numbers, etc. The definition of trees is equivalent. We will think of a trunk, which is the principle node, as the set $X$. Every branch on that trunk is an element of the set $X$. For example, a single trunk with no branches is the set number 0 . Suppose we know what tree $X$ is, how do we find the tree corresponding to $X \oplus 1$ ? We add a branch that is a 0 -tree (add 1 unit). So the set number 1 is a trunk with one 0 -branch because $1=\{0\}$; this branch has to be the tree representing 0. We can see this in Figure 6

A tree is a graph of nodes and edges such that (i) We can identify a trunk: a principle edge with a finite number of branches attached to one of the nodes. All branches are attached to the same node of the trunk. (ii) Each branch on the tree is a tree. (iii) A single edge is a tree; we call it the 0-tree. The successor of a tree is obtained by adding a single edge to the trunk; attach a 0 -tree to the trunk. Adding an edge to the 0-tree gives its successor, the 1-tree, which is two edges joined together at one node. Adding an edge to the 1-tree, we find its successor, the 2-tree, etc.

We need an extra rule for defining an equivalence class on finite trees. If a tree has two branches that are identical we substitute these two identical branches with a single branch, the successor. This process is called reduction. If a tree can be reduced to obtain another tree, they are in the same equivalence class. An irreducible tree is said to be in canonical form. Reducing the 2-tree, we find the canonical form. To reduce the 2-tree we substitute the two identical 0-trees with a single 1-tree. Adding a single edge to the result of that, we obtain the canonical form of the 3-tree in canonical form because there are no identical branches. If we add an edge to the 3-tree we have to reduce and obtain the canonical form of the 4-tree. We have to apply reduction of branches, two times. We first take away the two 0-trees and add a 1-tree. But, we already had a 1 -tree so now we have two identical 1-trees. We take those trees away and add a 2-tree. Every natural number is associated an equivalence class of finite trees, and a single canonical tree. Every branch on the canonical tree of a set number $X$ corresponds to a natural number $k \in X$. Every tree is made up of smaller trees, and we give a well defined method of building trees. The canonical tree corresponding to the set number $X$ has $\#(X)$ branches. Each branch is defined in the same way. A natural number is defined by its cardinality; and the cardinality of its elements; and the cardinality of the elements of its' elements; etc.

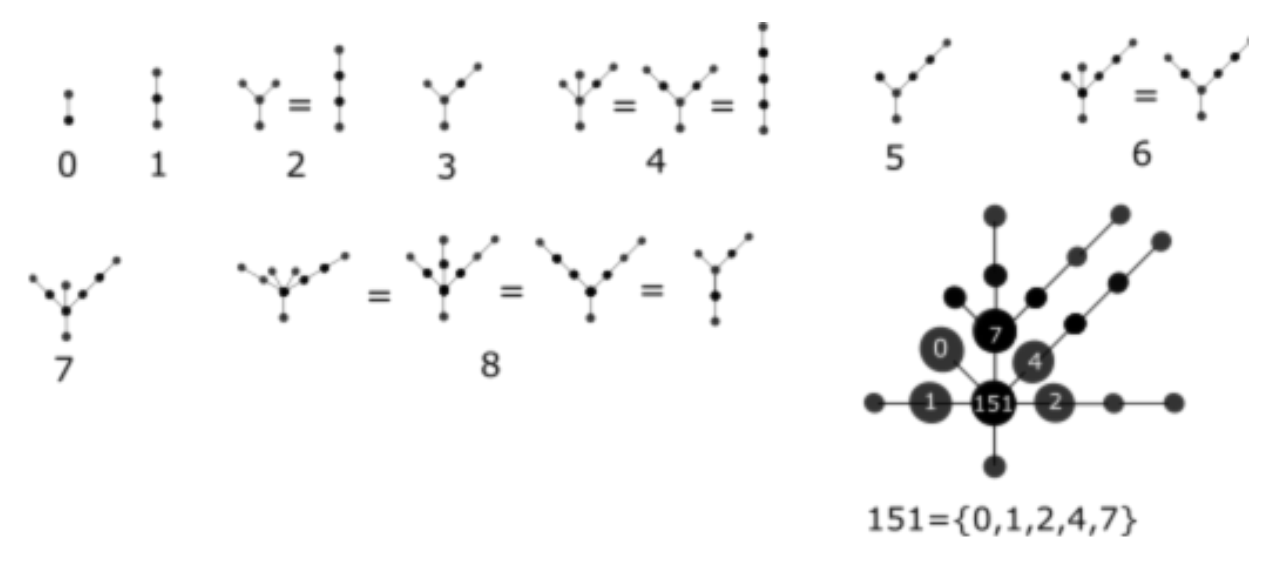

Figure 6: Canonical trees can be built easily, given a set number. The canonical tree for $7=\{0,1,2\}$ has three branches. One branch is the 0 -tree, the second branch is the 1 -tree and the third branch is the 2 -tree. The canonical tree of $8=\{3\}$ is a trunk with one branch, which is the 3-tree. The canonical tree of $151=\{0,1,2,4,7\}$ has five branches: 0,1,2,4,7-trees. 
Now we can use trees to represent real numbers. We simply use trees with infinite many branches. Each branch must be a finite tree and we do not allow these to be repeated. If the two branches are identical, we reduce the tree. Consider a new kind of tree, with infinite many branches. But, each of these branches is a tree of infinite branches. This is a collection of real numbers. In this next sub section we formalize this concept of types.

\subsection{Type Theory}

Finite trees are what we will call objects of Type-0. Trees of infinite branches with each branch being an object of type- 0 are called objects of Type-1. For example, a real number is an object of Type- 1 and a natural number is an object of Type-0. A tree whose branches are all objects of Type- 1 is an object of Type-2. An example of an object of Type-2 is a set of real numbers. A tree with branches of Type-0 and Type- 1 is an object of Type-3. This would be an object that has two types of elements. For example, a set consisting of natural and real numbers is an object of Type-3. An object of Type-4 is a tree with all its branches being objects of Type-2. An example of a Type-4 object is a family of sets of real numbers. For example, a collection of subsets of $\mathbb{R}$ is an object of Type-4. In general, we build the Type- $n$ objects in the same manner we build natural numbers.

The next step in classifying types of objects is to consider trees with infinite many types of branches. This means our tree has branches of Type- $n_{1}$, Type- $n_{2}$, Type- $n_{3} \ldots$ for infinite many types. This is called an object of infinite Type-1,0. An object of infinite Type-1,1 is a tree that only has branches of infinite Type-1,0. An object of Type-1,2 is a tree with all branches of Type-1,1. A tree with branches of both Type-1,0 and infinite Type-1,1 is an object of infinite Type-1,3. If all the branches of a tree are objects of Type-1,2, we say it is an object of infinite Type-1,4. We can construct all infinite Types-1, $k$.

Consider a tree whose branches are all objects of infinite type, and suppose there are infinite many types of objects of infinite type. We have objects of Type- $1, n_{1}$, Type- $1, n_{2}$, Type- $1, n_{3}, \ldots$. A tree built with objects of infinite many infinite-types, is an object of infinite Type-2,0. Trees whose objects are only objects of Type-2,0 are called objects of Type-2,1. A tree with all objects of Type-2,1 is an object of Type-2,2. A tree with objects of Type-2,0 and Type-2,1 is an object of Type-2,3, etc.

Now we have objects of infinite Type-3,0, which are trees whose branches are of finite type and infinite type. A tree with objects of Type-3,0 is an object of Type-3,1, and so on. An object of infinite Type-4,0 is a tree with infinite many types of objects of Type- $2, k$. This means an object of Type-4,0 has objects of Type- $2, n_{1}$, Type- $2, n_{2}$, Type- $2, n_{3} \ldots$ for infinite many types $2, k$. Of course an objects of Type 4, 1 is a tree with branches of Type-4,0, etc. An infinite Type-5,0 object consists of branches of finite type and types $2, k$. A Type-6,0 object consists of objects of types $1, k$ and types $2, k$, etc. We continue in this manner until we have built all objects of Type- $m, n$, for every $m, n \in \mathbb{N}$. And, leave it at this for now.

\section{Conclusions}

The importance of the axiomatic base is usually undermined because it does not bring any new results or methods into most practical areas of mathematics. Instead, the axiomatic base of mathematics is seen as a stone in the path; an obstacle to be dealt with and forgotten. The axiomatic base we provide here differs from others in the fact that we acquire natural constructions for classic structures of mathematics. The construction we provide of natural numbers allowed a natural description of finite structures. Then we extended our methods to describe infinite mathematical objects. More results can be pursued in future work. This can include a thorough description of groups, rings, fields and linear spaces, in the finite and infinite cases. This work has only served as introduction of these set theoretic results in the area of finite groups. Another line of work will include a description of the calculus of real numbers. Revisions on the theory of types and the Continuum Hypothesis are also in order. 
Algebraically, we are describing finite groups using natural numbers, in such a way that we have a good criteria for distinguishing finite groups. We can give a linear order, isomorphic to $\mathbb{N}$, to set of all finite groups. This linear order of groups is well behaved with respect to cardinality. This order on finite groups organizes groups amongst each other. But, we are also provided with a method for organizing finite groups, internally. We can also order the elements of any finite group in the canonical naming function. We have a criteria for defining equivalent objects in a group. Apart from this, we are also given a minimum set of independent equations that defines a group. Finding all finite groups of $n$ objects is still not trivial but we have a better notion of attacking this problem. We give an algorithm for proving isomorphism of two groups. We must build the canonical representation of both groups, and they should be the same natural number. Or simply put, the numerical table of the groups should be identical.

Computational aspects can also be treated with detail. This can also be done with a focus on finding physical methods in the area of Particle Physics, to represent the arithmetic of Energy Levels. If this can be done, it could have applications in modern computing. There are a variety of ways for codifying the information of mathematical structures, and we have provided the data types for some structures, although this library of types must be completed. We briefly discuss the most general case, where we are using trees to represent any type of mathematical object. In most cases we give the constructions of smallest type possible. For example, we give real functions the same data type as a set of real numbers. 


\section{References}

[Ramirez(2019)] Ramírez, J.P. A New Set Theory for Analysis.Axioms 2019, 8, 31.

[Ramirez(2015)] Ramírez, J.P. Systems and Categories. arXiv 2015, arXiv:1509.03649v5.

[Bernays(1991)] Bernays, P. Axiomatic Set Theory; Dover: New York, NY, USA, 1991.

[Benacerraf(1965)] Benacerraf, P. What Numbers Could Not Be. Philos. Rev. 1965, 74, 47-73.

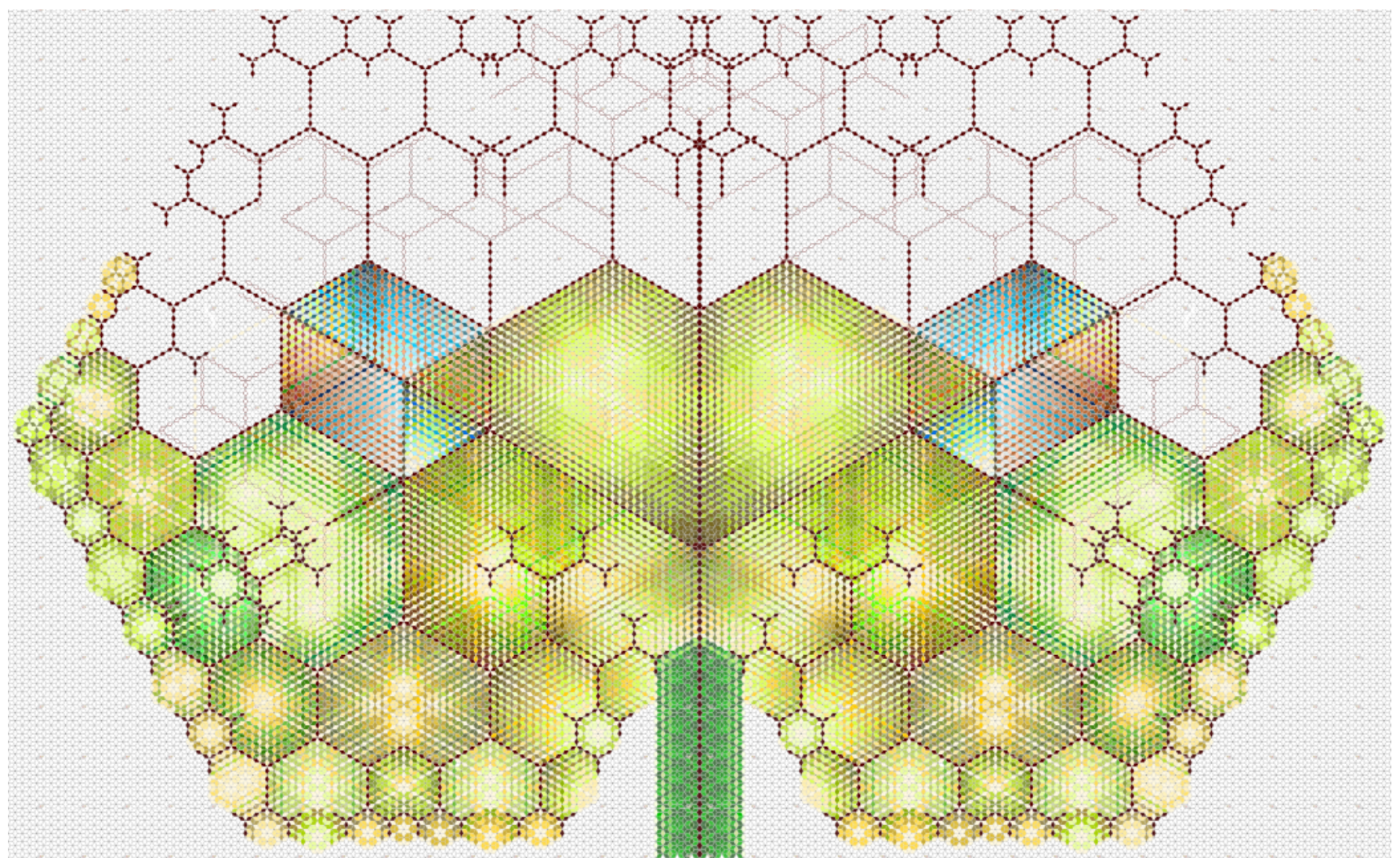

\#MuralInteligente 